\title{
Modeling and Urban Search Experiments: Fort Indiantown Gap Data Collections Summary and Analysis
}

Approved for public release. Distribution is unlimited.
Daniel E. Archer Mark S. Bandstra Gregory G. Davidson Steven L. Cleveland Irakli Garishvili Donald E. Hornback Jeffrey O. Johnson M. S. Lance McLean Andrew D. Nicholson Bruce W. Patton Douglas E. Peplow Alexander A. Plionis Brian J. Quiter William R. Ray Andrew J. Rowe Mathew W. Swinney Michael J. Willis

October 2017 


\title{
DOCUMENT AVAILABILITY
}

Reports produced after January 1, 1996, are generally available free via US Department of Energy (DOE) SciTech Connect.

Website http://www.osti.gov/scitech/

Reports produced before January 1, 1996, may be purchased by members of the public from the following source:

\author{
National Technical Information Service \\ 5285 Port Royal Road \\ Springfield, VA 22161 \\ Telephone 703-605-6000 (1-800-553-6847) \\ TDD 703-487-4639 \\ Fax 703-605-6900 \\ E-mail info@ntis.gov \\ Website http://classic.ntis.gov/
}

Reports are available to DOE employees, DOE contractors, Energy Technology Data Exchange representatives, and International Nuclear Information System representatives from the following source:

Office of Scientific and Technical Information

PO Box 62

Oak Ridge, TN 37831

Telephone 865-576-8401

Fax 865-576-5728

E-mail reports@osti.gov

Website http://www.osti.gov/contact.html

This report was prepared as an account of work sponsored by an agency of the United States Government. Neither the United States Government nor any agency thereof, nor any of their employees, makes any warranty, express or implied, or assumes any legal liability or responsibility for the accuracy, completeness, or usefulness of any information, apparatus, product, or process disclosed, or represents that its use would not infringe privately owned rights. Reference herein to any specific commercial product, process, or service by trade name, trademark, manufacturer, or otherwise, does not necessarily constitute or imply its endorsement, recommendation, or favoring by the United States Government or any agency thereof. The views and opinions of authors expressed herein do not necessarily state or reflect those of the United States Government or any agency thereof. 
Nuclear Materials and Detection Characterization Group

\title{
MODELING AND URBAN SEARCH EXPERIMENTS: FORT INDIANTOWN GAP DATA COLLECTIONS SUMMARY AND ANALYSIS
}

\author{
Daniel E. Archer ${ }^{1}$ \\ Mark S. Bandstra ${ }^{3}$ \\ Gregory G. Davidson ${ }^{1}$ \\ Steven L. Cleveland ${ }^{1}$ \\ Irakli Garishvili ${ }^{1}$ \\ Donald E. Hornback ${ }^{4}$ \\ Jeffrey O. Johnson ${ }^{1}$ \\ M. S. Lance McLean ${ }^{2}$ \\ Andrew D. Nicholson ${ }^{1}$ \\ Bruce W. Patton ${ }^{1}$ \\ Douglas E. Peplow ${ }^{1}$ \\ Alexander A. Plionis ${ }^{2}$ \\ Brian J. Quiter ${ }^{3}$ \\ William R. Ray ${ }^{1}$ \\ Andrew J. Rowe ${ }^{5}$ \\ Mathew W. Swinney ${ }^{1}$ \\ Michael J. Willis ${ }^{1}$
}

\footnotetext{
${ }^{1}$ Oak Ridge National Laboratory, Oak Ridge, TN 37831 USA

${ }^{2}$ Remote Sensing Laboratory, Washington DC 20762 USA

${ }^{3}$ Lawrence Berkeley National Laboratory, Berkeley, CA, 94720 USA

${ }^{4}$ Department of Energy, Washington DC USA

${ }^{5}$ Cadre5, Knoxville, TN 37932 USA
}

Date Published: October 2017

\author{
Prepared by \\ OAK RIDGE NATIONAL LABORATORY \\ Oak Ridge, TN 37831-6283 \\ managed by \\ UT-BATTELLE, LLC \\ for the \\ US DEPARTMENT OF ENERGY \\ under contract DE-AC05-00OR22725
}





\section{CONTENTS}

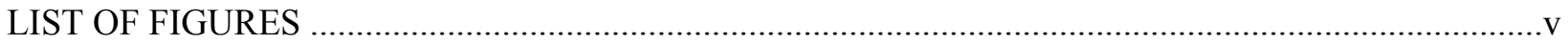

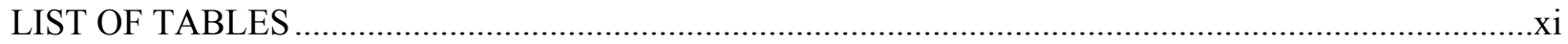

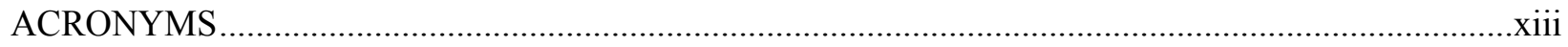

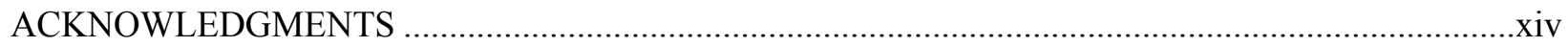

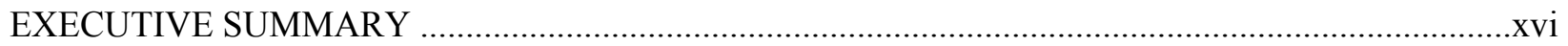

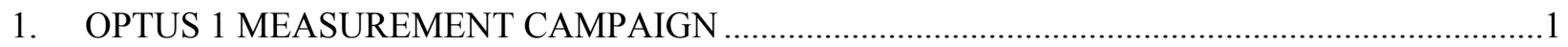

1.1 HPGE OUTDOOR BUILDING AND GROUND SURFACE MEASUREMENTS ..................1

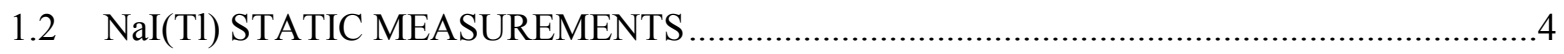

$1.3 \mathrm{NaI(Tl)}$ DYNAMIC BACKGROUND MEASUREMENTS …...........................................

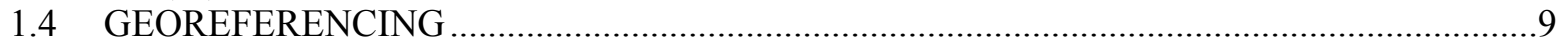

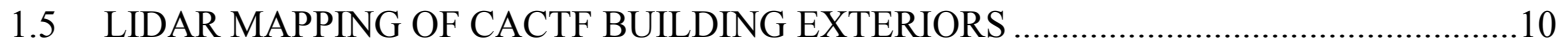

1.6 METEOROLOGICAL AND PERSISTENT RADIATION MONITORING ........................13

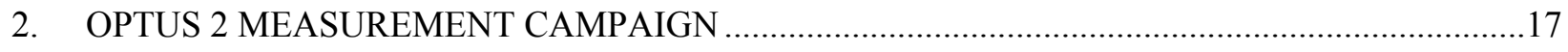

2.1 HPGe OUTDOOR BUILDING AND GROUND SURFACE MEASUREMENTS.................17

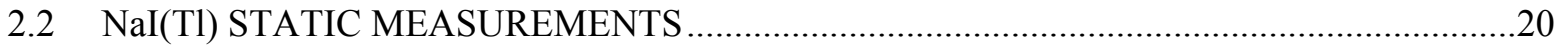

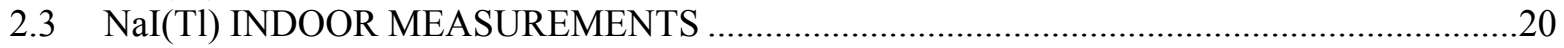

2.4 DIRECTIONAL NaI(Tl) BACKGROUND SOURCE TERM MEASUREMENTS ................23

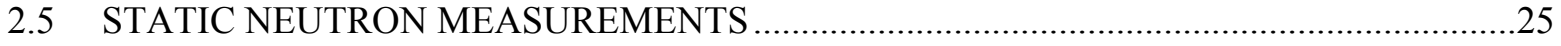

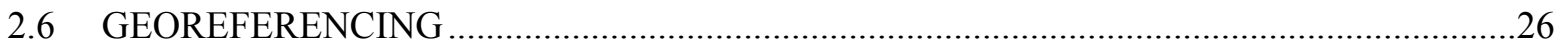

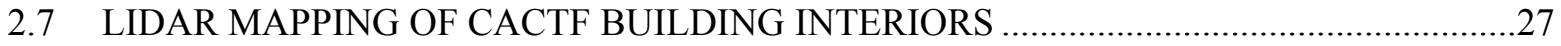

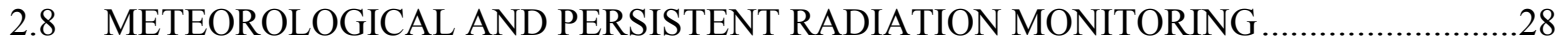

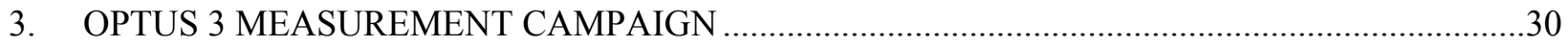

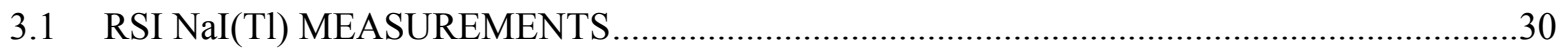

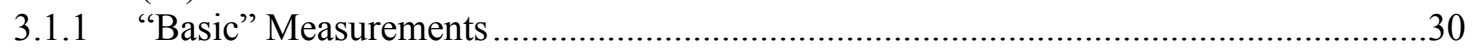

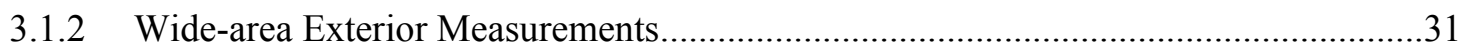

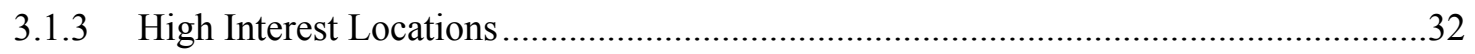

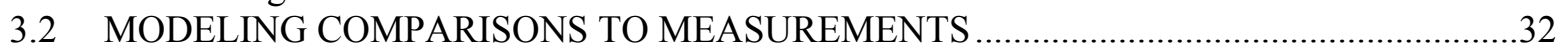

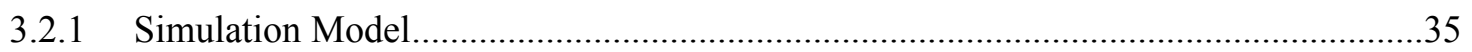

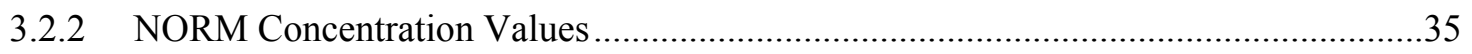

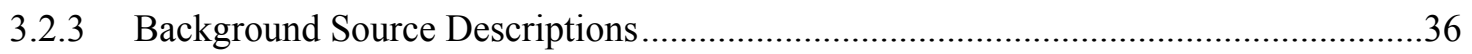

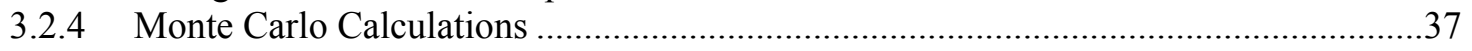

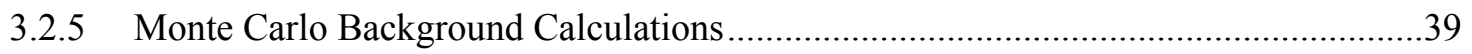

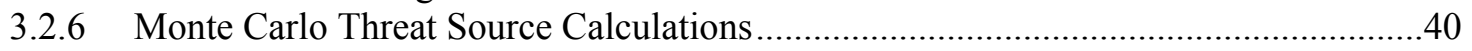

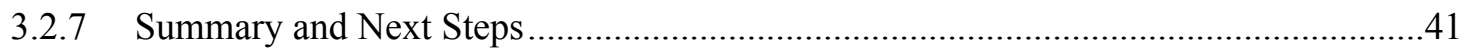

4. MUSE 1 MEASUREMENT CAMPAIGN .....................................................................................45

4.1 HPGe OUTDOOR BUILDING AND GROUND SURFACE MEASUREMENTS.................45

$4.2 \mathrm{NaI(T1)} \mathrm{STATIC} \mathrm{BACKGROUND} \mathrm{MEASUREMENTS} \mathrm{...................................................50}$

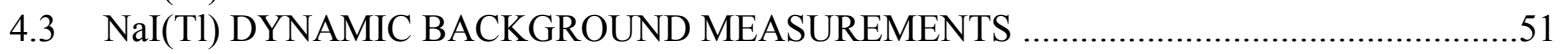

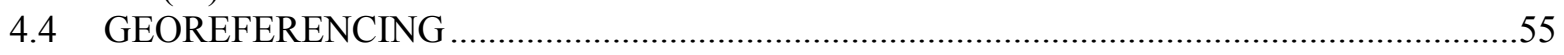

4.5 LIDAR/VISUAL MAPPING OF CACTF BUILDING EXTERIORS ...................................55

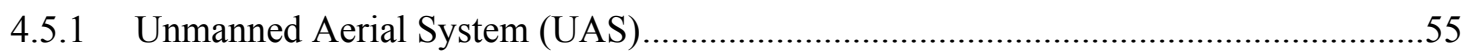

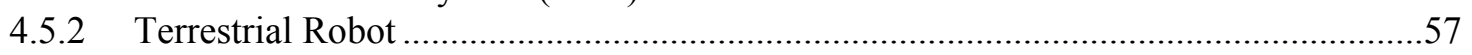

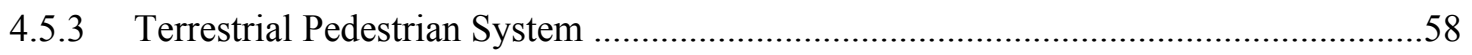

4.6 METEOROLOGICAL AND PERSISTENT RADIATION MONITORING .........................58

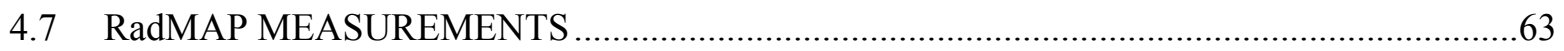

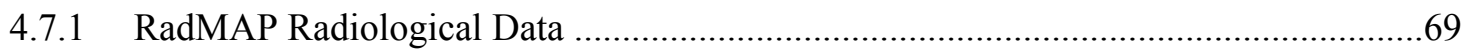

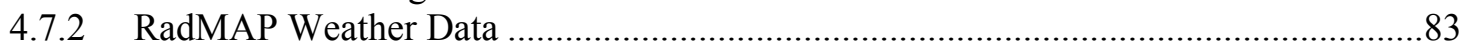

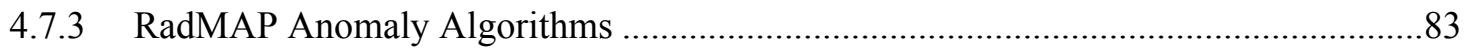




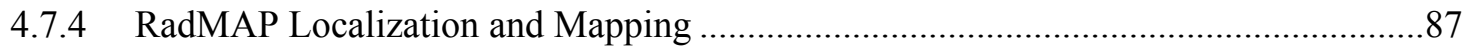

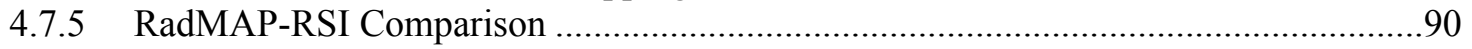

4.7.6 RadMAP Radiological Inversion Progress ...............................................................94

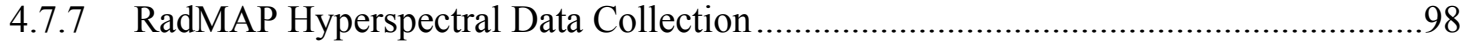

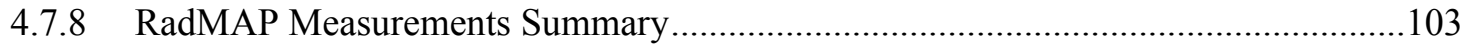

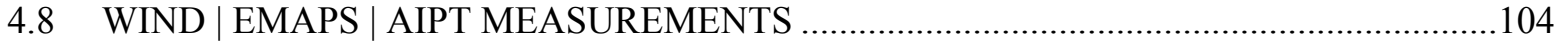

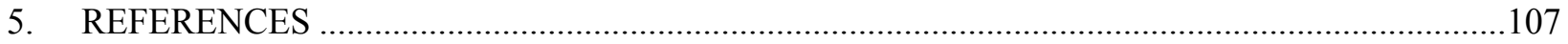




\section{LIST OF FIGURES}

Figure 1.1.1: (Top left) Photograph of a HPGe measurement. The detector is facing the cinder block wall. (Top right) Correlation between $1,460 \mathrm{keV}{ }^{40} \mathrm{~K}$ and $2,614 \mathrm{keV}{ }^{232} \mathrm{Th}\left({ }^{208} \mathrm{Tl}\right)$ peak areas for the OPTUS 1 Measurement Campaign. (Bottom left) Correlation between $1,460 \mathrm{keV}{ }^{40 \mathrm{~K}}$ and $609 \mathrm{keV}{ }^{214} \mathrm{Bi}$ peak areas. (Bottom right) Correlation between $2,614 \mathrm{keV}{ }^{232} \mathrm{Th}\left({ }^{208} \mathrm{Tl}\right)$ and $609 \mathrm{keV}{ }^{214} \mathrm{Bi}$ peak areas.

Figure 1.2.1. OPTUS 1 static measurement results. (Top left) Photograph of $\mathrm{NaI}(\mathrm{Tl})$ detector in a low scatter cart.

Figure 1.2.2. Illustration of mobile detection methodology using static measurements. (Above) Interpolation procedure used to generate synthetic data from static measurements.

Figure 1.2.3. Example of detector response interpolation. Solid markers denote measured total count rates, and empty markers show interpolated spectra. Change in count rate from $53.5 \mathrm{~m}$ to $54 \mathrm{~m}$ is due to measurements being performed with different detector units

Figure 1.2.4. Sampling procedure used to generate synthetic data. (Left) An interpolated detector response. The inset shows a Poisson distribution of a channel in the detector. (Right) A sample for a detector integration time of $0.5 \mathrm{~s}$..

Figure 1.2.5. Example of detector response sampling. Cyan curve shows the original detector response (one taken over infinite time). The red curve represents a detector response for a $1 \mathrm{~h}$ integration time, the green for $1 \mathrm{~min}$. and the blue for $1 \mathrm{~s}$.

Figure 1.3.1. OPTUS 1 dynamic NaI(Tl) measurements at CACTF. (Top left) Van GPS locations for three loops around the CACTF. (Top right) Van total count rates for the three passes shown in the top left. (Bottom left) Walking GPS locations for one pass along Main St. (Bottom right) Total count rates for the pass shown in the bottom left figure................................

Figure 1.4.1. Cartesian layout of the CACTF.

Figure 1.4.2. Differential GPS points for all NaI(T1), LIDAR, HPGe, and meteorology collection locations.

Figure 1.5.1. DGPS unit employed during measurement campaign. (A) $\mathrm{Z}+\mathrm{F}$ model 5006 LIDAR.

$(B)$ Example of stationary and nonstationary targets used in LIDAR collection.

Figure 1.5.2. Optical images showing different perspectives of a LIDAR scan position.

Figure 1.5.3. LIDAR image and measurement locations created of CACTF. (Left) Composite point cloud of FTIG with high-resolution LIDAR. (Right) Locations where individual scans were taken are marked.

Figure 1.5.4. CAD model created of CACTF using OPTUS 1 imaging measurements. $(A)$ CAD model manually derived from ground truth point cloud. $(B)$ Spatially resolved comparison of the mismatch between the point cloud and the CAD model.

Figure 1.6.1. Weather station locations for OPTUS 1 campaign

Figure 1.6.2. RSI Detector count rate versus air temperature (above) and count rate versus rainfall (below).

Figure 1.6.3. Spectrums collected during May 16 rain event. (Left) Counts per 5 min. versus time zoomed. (Right) Raw gamma-ray spectra before, during, and after the rain.

Figure 1.6.4. Spectrums collected during May 18 rain event. (Left) Counts per 5 min. versus time zoomed. (Right) Raw gamma-ray spectra before, during and after the rain.

Figure 2.1.1. (Top left) A picture of one of the HPGe measurements conducted as part of the OPTUS 2 .

Figure 2.2.1. Static background count rate comparison for OPTUS 1 (empty black circles) and OPTUS 2 (filled red) for the same positions down Main St.

Figure 2.3.1. Detector (black) and source (red) locations for point throughout the hotel. (a) Basement, (b) first floor, (c) second floor, (d) third floor, and (e) roof. R\# denotes the sensor position, S\# denotes the source position. 
Figure 2.3.2. Stairwell detector response comparisons for source location S1. ....................................22

Figure 2.3.3. Near-elevator detector response comparisons for source location S2 ...............................22

Figure 2.4.1. Configurations for the shielded $\mathrm{NaI}(\mathrm{Tl})$ measurements. ................................................23

Figure 2.4.2. Measurement configuration examples for shielded directional $\mathrm{NaI}(\mathrm{Tl})$

measurements

Figure 2.4.3. Comparison of the spectra from the wholly unshielded spectrum (black) and the unshielded top, bottom, left, right, and tip (blue)

Figure 2.4.4. Comparison of the spectra from the wholly unshielded spectrum (black) and the unshielded top, bottom, left, right, and tip x 2 (blue).

Figure 2.5.1. Static neutron detection system measurements along Main St. (Left) Photograph of ${ }^{3} \mathrm{He}$ detection system. (Right) Total neutron counts per panel per second versus position

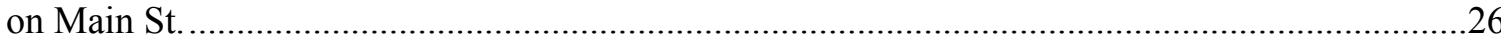

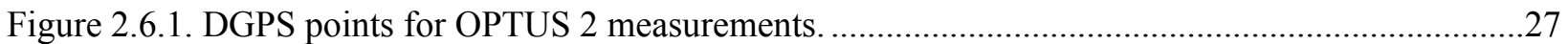

Figure 2.7.1. LIDAR-generated point clouds for the hotel on CACTF. (a) Basement, (b) First floor, (c) Second floor, (d) Third floor, (e) Roof, and (f) Hotel exterior......................................27

Figure 2.7.2. Combined LIDAR point cloud of interior and exterior of hotel..........................................28

Figure 2.8.1. Detector response with two relevant ROI $5 \mathrm{~min}$. counts compared to air temperature..........29

Figure 3.1.1. Example cinder block construction and measurement target locations................................30

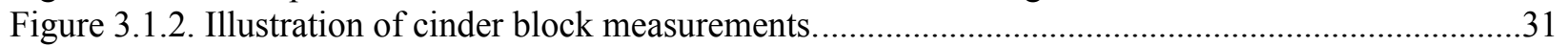

Figure 3.1.3. The high-interest location measurement geometry. .........................................................32

Figure 3.2.1. Overhead view of the main street of the FTIG CACTF showing the source locations (red circles, labeled 1-4) and detector locations, in meters (gray circles, labeled 10-80)............33

Figure 3.2.2. Photograph of two detector systems outside high-interest location. ......................................34

Figure 3.2.3. Measured spectra for detector location $\mathrm{x}=30 \mathrm{~m}$. Background only (blue, upper left), with the cesium source (gray, lower left) and both spectra near the $662 \mathrm{keV}$ region (right)

Figure 3.2.4. The full FTIG CACTF model with nine buildings, concrete intersections (white),

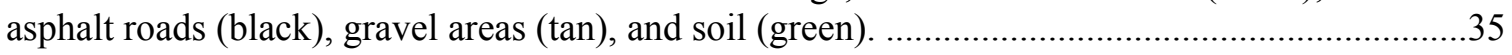

Figure 3.2.5. An example energy-dependent flux tally from SCALE/MAVRIC .....................................38

Figure 3.2.6. The pulse-height distribution from the flux computed at the detector location using

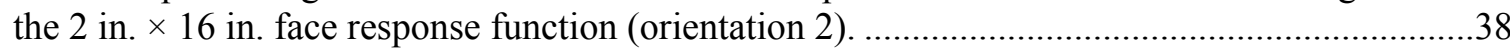

Figure 3.2.7. The predicted energy spectrum (linear scale) for a $\mathrm{NaI}(\mathrm{Tl})$ (orientation 2). (GADRAS non-HPGe values P6 $=0, \mathrm{P} 7=7.5$, and P8 $=0.7$ )

Figure 3.2.8. Predicted energy spectrum (logarithmic scale) for a $\mathrm{NaI}(\mathrm{Tl})$ (orientation 2).

(GADRAS non-HPGe values P6 $=0, \mathrm{P} 7=7.5$, and P8 $=0.7$ ).

Figure 3.2.9. Simulated and measured background spectra (linear scale) at $\mathrm{x}=30 \mathrm{~m}$. Measurements (cyan) include the $1 \sigma$ uncertainties and the simulated results (red) show two detector responses (the 2 in. $\times 16$ in. face is the lower red line and the 4 in. $\times 16$ in. face is the upper red line).

Figure 3.2.10. Simulated and measured background spectra (logarithmic scale) at $\mathrm{x}=30 \mathrm{~m}$. Measurements (cyan) include the 1- $\sigma$ uncertainties and the simulated results (red) show two detector responses (the 2 in. $\times 16$ in. face is the lower red line and the 14 in. $\times 16$ in. face is the upper red line)...

Figure 3.2.11. Comparison of measured (cyan) and simulated (red) detector responses for WAE1 at $10 \mathrm{~m}$. (Left) the full spectrum with cesium source present and simulated background (magenta). (Right) Background-subtracted spectra and the two simulated responses.

Figure 3.2.12. Comparison of measured (cyan) and simulated (red) detector responses for WAE1 at $20 \mathrm{~m}$. (Left) The full spectrum with cesium source present and simulated background (magenta). (Right) The background-subtracted spectra and the two simulated responses.

Figure 3.2.13. Comparison of simulated (red) and measured values of background subtracted (blue) counts for source position 1 and detector location $\mathrm{x}=30 \mathrm{~m}$. 
Figure 3.2.14. Comparison of simulated (red) and measured values of background subtracted (blue) counts for source position 1 and detector location $\mathrm{x}=30 \mathrm{~m}$. This is the same data as Figure 3.2.13 but with larger bins for measured data.

Figure 4.1.1. An example spectrum obtained from a soil measurement with regions containing peaks of interest highlighted in red.

Figure 4.1.2. Comparison of the ORTEC EX-100T detector surrounded by lead for measurement H47 (left) to the MCNP6 model developed for this effort (right).

Figure 4.1.3. Example of an MCNP6 simulation designed to predict the contribution from background sources beyond a CMU wall (left) and the actual experimental setup (right)

Figure 4.1.4. A side view of the MCNP6 simulation geometry used to estimate the detector response through a CMU wall (red, left) from a distributed source in soil (blue), sidewalk (grey), and asphalt pavement (orange)...

Figure 4.1.5. NORM concentrations of 70 material measurements across FTIG. Note that the ticks are not error bars but represent the bounds of the determined NORM values for each material based on individual measurements.

Figure 4.2.1. OPTUS 1 and MUSE 1 measurement comparisons. (Top left) MUSE 1 static measurements compared with OPTUS 1 measurements. (Top right) ${ }^{214} \mathrm{Bi},{ }^{40} \mathrm{~K}$, and ${ }^{232} \mathrm{Th}$ peak areas for OPTUS 1 (empty markers) and MUSE 1 (filled markers). (Bottom) MUSE 1 static measurements and Monte Carlo particle transport detector response function calculations. Although the predicted Monte Carlo response functions closely track the variance in the static measurements, the magnitude is less.

Figure 4.3.1. MUSE 1 static, dynamic, and synthetic data comparison. (Left) Path of moving 2 in. $\times 4$ in. $\times 16$ in. $\mathrm{NaI}(\mathrm{Tl})$ detector down Main St. at $1.23 \mathrm{~m} / \mathrm{s}$. (Right) Total count rate of static measurements, dynamic measurement and synthetic data generated from the static data.

Figure 4.3.2. MUSE 1 measured static and dynamic datasets compared to calculated synthetic data. (Left) Comparison of static, dynamic and synthetic datasets. Gold markers within the red dataset denote static measurements used for synthetic dataset generation. (Right) Normalized histogram of difference between dynamic and synthetic datasets using varying amounts of static measurements in synthetic data generation.

Figure 4.3.3. Average static/dynamic difference with $1-\sigma$ error. After changing the difference between positions used for interpolation is $>30 \mathrm{~m}$, a shift in the synthetic difference mean is observed. For an environment with larger background variations, this distance will decrease.

Figure 4.4.1. Locations of MUSE 1 HPGe measurements.

Figure 4.5.1. UAS visible and thermal imaging system. (Left) UAS system and sensors used for data acquisition. (Middle) Example of visual sensor data collected with the UAS. (Right) Example of a flight plan used for a collection. Alternate paths followed the perimeter of FTIG.

Figure 4.5.2. Point cloud generated from a combination of visible photographs and UAS platform.

Figure 4.5.3. Comparison of UAS point cloud versus point cloud from long-dwell LIDAR collected during FY 2015 campaigns.

Figure 4.5.4. Terrestrial robot acquisition system with LIDAR and $360^{\circ}$ camera system coupled to radiation detection cart

Figure 4.6.1. WPRM-2 with Davis weather station and two NaI(Tl) detectors. ....................................59

Figure 4.6.2. Persistent weather monitoring station locations.

Figure 4.6.3. MUSE 1 static measurement comparison along Main St. (Left) Static background comparison along Main St. for different times of the day and (right) the count rate difference between measurement times. 
Figure 4.6.4. (Above) Persistent background monitor gross count rates versus time and (below) count rates versus time for the ${ }^{214} \mathrm{Bi} \mathrm{ROI}$.

Figure 4.6.5. Count rates versus time for ${ }^{40} \mathrm{~K}$ ROI and ${ }^{232} \mathrm{Th} \mathrm{ROI}$.

Figure 4.6.6. Comparison of environmental variables and count rate over time for persistent monitoring station.

Figure 4.6.7. Count rate versus weather variables between 12:00 p.m. and 6:00 p.m. on September 2.

Figure 4.7.1. Illustration of RadMAP system with an overlaid drawing indicating the positions of the fielded sensors.

Figure 4.7.2. RadMAP dynamic measurements through FTIG. (Left) Top-down view of RadMAP Day 1 survey paths.

Figure 4.7.3. RadMAP Speed profiles along Main St. for initial 29 loops. The curves are colorized by relative time, with the first 10 uphill nominal $5 \mathrm{mph}$ loops in blue, then seven $10 \mathrm{mph}$ loops in green-yellow, then uphill $15 \mathrm{mph}$ in yellow/red, followed by the downhill $5 \mathrm{mph}$ loops in red. The abscissa is linear distance along Main St., relative to a point approximately at the center of the northern intersection.

Figure 4.7.4. RadMAP static measurement locations. (Left) Locations of Day 1 static measurements.

Figure 4.7.5. RadMAP loops through FTIG. (Left) RadMAP Day 2 time-perspective path.

Figure 4.7.6. Photograph of RadMAP static measurement process. (Left) Measurement RC1, (center) measurement RH1, (left) photograph of step being measured for positioning. The left and center photographs are indicative the left-right range of RadMAP positioning relative to the roadway curbs (where present).

Figure 4.7.7. Example 3 h dynamic RadMAP spectrum collected Day 1. (Left) RadMAP summed $\mathrm{NaI}(\mathrm{Tl})$ array spectrum from $\sim 3 \mathrm{~h}$ of Day 1 dynamic measurements.

Figure 4.7.8. Time- and position-dependent $1 \mathrm{~s}$ count rates from RadMAP NaI(Tl) array. (Top left) Gross counts, (Top right) ${ }^{40} \mathrm{~K}$ peak ROI, (Bottom left) uranium ROI 1,720-2,450 keV, and (Bottom right) ${ }^{208} \mathrm{Tl}$ peak ROI. For the ROI, events attributed to background were subtracted as described in the text.

Figure 4.7.9. Spatially aggregated $\mathrm{NaI}(\mathrm{Tl})$ RadMAP array rates into a $3 \mathrm{~m}$ grid. The top left figure indicates the time spent in each grid point (and is cropped at $40 \mathrm{~s}$ ). Grid points with times greater than $10 \mathrm{~s}$ were considered for potassium (middle left), uranium (middle right) and thorium (bottom), but all times were considered for gross counts (top right).

Figure 4.7.10. Time-and position-dependent $1 \mathrm{~s}$ count rates for the RadMAP IDM HPGe array. (Top left) Gross counts $>70 \mathrm{keV}$, (top right) ${ }^{40} \mathrm{~K}$ peak, (bottom left) four ${ }^{214} \mathrm{Bi}$ peaks due to uranium-series decays, and (bottom right) ${ }^{208} \mathrm{Tl}$ peak.

Figure 4.7.11. Spatially aggregated RadMAP IDM array rates into a $3 \mathrm{~m}$ grid. Only grid points with times greater than $10 \mathrm{~s}$ were considered for ${ }^{40} \mathrm{~K}$ (top right), four ${ }^{214} \mathrm{Bi}$ peak due to the uranium-series decays (bottom left) and ${ }^{208} \mathrm{Tl}$ due to thorium decay (bottom right). All times were considered for gross counts, which had a $70 \mathrm{keV}$ threshold applied (top left).....

Figure 4.7.12. Comparison of RadMAP NaI(Tl) and IDM array. (Left) RadMAP NaI(Tl) and IDM (Right) array gross count rates for each uphill, east-side loop along Main St. binned into $2 \mathrm{~m}$ bins along the dimension of Main St.

Figure 4.7.13. Liquid scintillator neutron detector Day 1 measurements. (Left) Gross LS count rates for 16 detectors.

Figure 4.7.14. PSD plots for 28 and 400 ns pulse integrations across RadMAP 16 LS detectors. (Left) PSD plot of the ratio of the maximum $400 \mathrm{~ns}$ pulse integration to a $28 \mathrm{~ns}$ pulse integration vs the $400 \mathrm{~ns}$ integral for all 16 detectors during the first 15 laps during Day 1 ..........78

Figure 4.7.15. RadMAP neutron count rate as a function of time during Day 1. (Left) The summed neutron count rate across the 16 detector array binned in $20 \mathrm{~s}$ bins for times 60 90 min. into the Day 1 laps. 
Figure 4.7.16. Dynamic RadMAP neutron measurements collected of FTIG. (Left) Spatially aggregated neutron count rates across the Day 1 measurements for pixels wherein greater than $10 \mathrm{~s}$ of data were collected.

Figure 4.7.17. RadMAP static NaI(Tl) Day 2 measurements. (Left) A series of histograms of the deviation normalized $\mathrm{NaI}(\mathrm{Tl})$ gross count rates relative to the measurement mean for 20 Day 2 static measurements as well as the combination of the with- and without-truck measurements of the northern field (green).

Figure 4.7.18. RadMAP integrated Day 2 gross counts from HPGe IDM array. (Top) IDM array gross counts as a function of Day 2 static measurement location. Locations are indicated on the abscissa.

Figure 4.7.19. RadMAP derived static LS neutron count rate. The circled measurement times are shown in further detail in Figure 4.7.20.

Figure 4.7.20. RadMAP Day 2 LS array static measurements. The statistical error associated with each independent data point (e.g., ones separated by $250 \mathrm{~s}$ ) ranges between 0.10 and 0.12 . (Below) Observed pressure readings during the same measurement interval.....

Figure 4.7.21. RadMAP MUSE 1 observed weather data.

Figure 4.7.22. RadMAP time-dependent anomaly measurements for both HPGe and NaI(Tl) arrays. Time-dependent anomaly metrics for (above) 21 isotopes as measured by the HPGe 3-window anomaly algorithm and (below) 22 isotopes as measured by the $\mathrm{NaI}(\mathrm{Tl})$ 3-window anomaly algorithm.

Figure 4.7.23. RadMAP 3-D map of anomaly measurement with algorithm score. (Left) 3-D plot of RadMAP location versus time, colorized by ${ }^{137} \mathrm{Cs} \mathrm{NaI}(\mathrm{Tl}) 3$-window algorithm anomaly score.

Figure 4.7.24. RadMAP scoring metric values for 3-window and PCS algorithms. Mean and standard deviation anomaly metric values for the 3-window algorithm (left) and for the PCS algorithm (right) plotted as a function of the isotope index given in Table 4.7.5.

Figure 4.7.25. RadMAP path and algorithm metric during ${ }^{137} \mathrm{Cs}$ anomaly. (Left) Logarithms of $\mathrm{NaI}(\mathrm{Tl})$ 137Cs PCS anomaly metrics plotted against time and position.

Figure 4.7.26. SLAM-derived point clouds of the FTIG CACTF facility. (Top left) Raw point cloud before SLAM self-consistency checks designed to removed transient features. (Top right) Same dataset after transient removal. (Bottom left) Top-down view of CACTF facility generated from 10 min. of RadMAP collection. (Bottom right) Same dataset in perspective view.

Figure 4.7.27. LIDAR values overlaid on RadMAP Ladybug $4 \pi$ still-frame image.

Figure 4.7.28. Analysis of RadMAP SLAM odometry algorithm. (Left) Horizontal distances between the INS and GPS solutions and the SLAM solutions that produce the minimal distance residuals after the time indicated by the black vertical line.

Figure 4.7.29. RadMAP path during SLAM algorithm measurements. Note, the maximum values

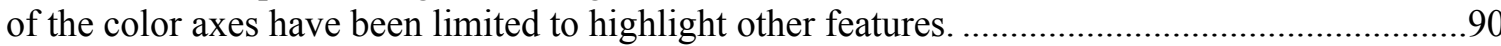

Figure 4.7.30. RadMAP and RSI total gross counts comparisons.

Figure 4.7.31. RadMAP and RSL gross count comparison along the path through FTIG. RadMAP system (left) and the RSL system (right).

Figure 4.7.32. RadMAP and RSI gross count and algorithm comparison along Main St. Comparisons between (left) gross counts for the RadMAP and RSI systems along Main St. for (top) the loop A uphill laps, (middle) the loop A downhill laps, and (bottom) the loop B downhill laps.

Figure 4.7.33: RadMAP panoramic image from port and starboard Ladybug cameras.

Figure 4.7.34. RadMAP imaging system automatic segmentation methodology. (Left) Stitched panoramic image.

Figure 4.7.35. Illustration of training image system with materials. (Top left) Panoramic image with overlaid manual labeling of objects within image. 
Figure 4.7.36. Illustration of pre- and post-foreground estimation for imaging system. (Left) Same still-frame as in Figure 4.7.35 with estimated foreground content. ....

Figure 4.7.37. Response for the indicated classes versus time for a single loop A lap through the FTIG facility.

Figure 4.7.38. Best-fit response of measured background and calculated from imaging.

Figure 4.7.39. Example mean spectrum observed in the hyperspectral imaging system. (Left) $2 \mathrm{i}$ camera mean spectrum in the FOV and the mean white-sky spectrum.

Figure 4.7.40. RadMAP NIR system output image. Mean image from 5,000 frames of NIR data while the system is moving is shown in the left figure.

Figure 4.7.41. RadMAP resulting single color image from camera systems. (Top left) Combined average reflectance of the two camera system.

Figure 4.7.42. Single value decompositions of measured spectrum with resulting wavelength. (Left) Eigenvalues for the first 20 principal components for the two SVDs.

Figure 4.7.43. False-color images for the eigen-images of the first twelve principal components of sky-facing SVD. The R,G,B values are the indicated principal components for each image.

Figure 4.7.44. False-color images for the eigen-images of the first nine principal components for the ground-facing SVD. The R,G,B values are the indicated principal components for each image.

Figure 4.8.1. DNDO WIND backpack radiation detection system prototype. 104

Figure 4.8.2. Measurement path for WIND data collections.

Figure 4.8.3. Count rates for measurements taken with the WIND backpack. 106 


\section{LIST OF TABLES}

Table ES-1.1.1. OPTUS 1 data collection summary. ...........................................................................

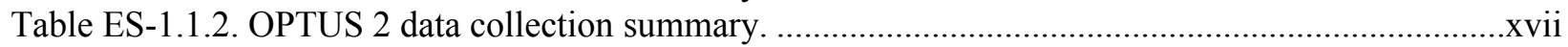

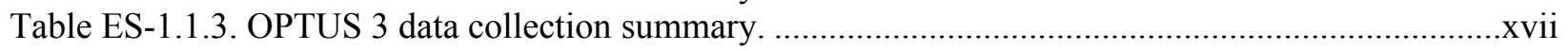

Table ES-1.1.4. MUSE 1 data collection summary.............................................................................

Table 1.1.1. Comprehensive list of OPTUS 1 HPGe measurements. .......................................................1

Table 2.1.1. Comprehensive list of OPTUS 2 HPGe measurements...................................................17

Table 2.4.1. Measurements with open face configurations and resulting count rates ...............................24

Table 3.2.1. Source and detector locations (Bold = direct line-of-sight to source $\mid \operatorname{Red}=$ no data

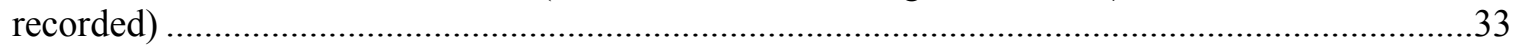

Table 3.2.2. Concentration of NORM for the major materials at the FTIG CACTF …...........................36

Table 4.1.1. Comprehensive List of MUSE HPGe Measurements......................................................45

Table 4.1.2. Calculated NORM activity concentrations for various materials at FTIG $(\mathrm{Bq} / \mathrm{kg})$...............49

Table 4.7.1. A summary of RadMAP measurements performed during MUSE-1 campaign. ...................65

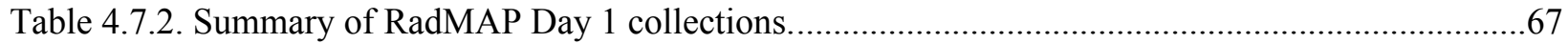

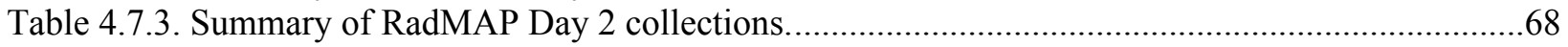

Table 4.7.4. Summary of RadMAP Day 3 collections............................................................................69

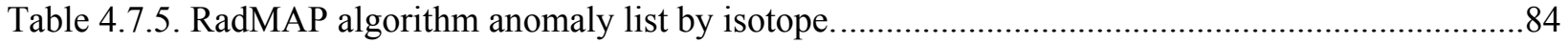





\section{ACRONYMS}

\begin{tabular}{|c|c|}
\hline CACTF & Combined Arms Collective Training Facility \\
\hline CCD & Charge Coupling Device \\
\hline CMU & Concrete Masonry Unit \\
\hline CPS & Counts per Second \\
\hline DGPS & Differential GPS \\
\hline DNDO & Domestic Nuclear Detection Office \\
\hline FOV & Field of View \\
\hline FTIG & Fort Indiantown Gap \\
\hline FWHM & Full Width at the Half Maximum \\
\hline FY & Fiscal Year \\
\hline GPS & Global Positioning System \\
\hline HIS & Hyperspectral Imagery \\
\hline HPGe & High-Purity Germanium \\
\hline IDM & Interchangeable Detector Modules \\
\hline IMU & Inertial Measurement Unit \\
\hline INS & Inertial Navigation System \\
\hline LIDAR & Light Detection and Ranging \\
\hline LS & Liquid Scintillators \\
\hline MCNP & Monte Carlo Neutral Particle \\
\hline MOUT & Military Operations on Urban Terrain \\
\hline NIR & Near Infrared \\
\hline NORM & Naturally Occurring Radioactive Materials \\
\hline ORNL & Oak Ridge National Laboratory \\
\hline PCS & Poisson Clutter Split \\
\hline PDT & Pacific daylight time \\
\hline RadMAP & Radiological Multisensor Analysis Platform \\
\hline $\mathrm{ROC}$ & Range Operation Center \\
\hline ROI & Region of Interest \\
\hline RSI & Radiation Solutions Inc. \\
\hline RSL & Remote Sensing Laboratory \\
\hline SLAM & Simultaneous Localization and Mapping Algorithm \\
\hline SLIC & Simple Linear Iterative Clustering \\
\hline SNM & Special Nuclear Material \\
\hline SUV & Sport Utility Vehicle \\
\hline SVD & Singular Value Decompositions \\
\hline UAS & Unmanned Aerial System \\
\hline UAV & Unmanned Aerial Vehicle \\
\hline WAE & Wide-area Exterior \\
\hline WIND & Wearable Intelligent Nuclear Detection \\
\hline WPRM & Weather/Persistent Radiation Monitoring \\
\hline
\end{tabular}




\section{ACKNOWLEDGMENTS}

The authors wish to thank James Peltz of the National Nuclear Security Administration Defense Nuclear Nonproliferation Office of Nonproliferation and Verification Research and Development for his support and patience while serving as the program manager. 



\section{EXECUTIVE SUMMARY}

This report summarizes experimental radiation detector, contextual sensor, weather, and global positioning system (GPS) data collected to inform and validate a comprehensive, operational radiation transport modeling framework to evaluate radiation detector system and algorithm performance. This framework will be used to study the influence of systematic effects (such as geometry, background activity, background variability, environmental shielding, etc.) on detector responses and algorithm performance using synthetic time series data. This work consists of performing data collection campaigns at a canonical, controlled environment for complete radiological characterization to help construct and benchmark a high-fidelity model with quantified system geometries, detector response functions, and source terms for background and threat objects. This data also provides an archival, benchmark dataset that can be used by the radiation detection community. The data reported here spans four data collection campaigns conducted between May 2015 and September 2016.

The first data collection campaign, OPTUS 1, was performed in May 2015 at the Combined Arms Collective Training Facility (CACTF) in Fort Indiantown Gap (FTIG), Pennsylvania, and aimed to collect all details necessary for model creation including radiation data, geometries, and metadata as accurately as possible. Sample background radiation levels for a variety of materials present at the site (including cinder block, gravel, asphalt, and soil) were collected using shielded, long dwell high-purity germanium (HPGe) measurements to quantify the contributing sources of naturally occurring radioactive materials (NORM). In addition to the shielded HPGe measurements, benchmark quality measurements using unshielded 2 in. $\times 4$ in. $\times 16$ in. $\mathrm{NaI}(\mathrm{Tl})$ detectors placed in carts were taken at a variety of locations around the FTIG site to validate model output. On-site persistent radiation and meteorological monitoring stations were used to monitor the possible change in radiation response due to potential weather phenomena. One station was placed on the CACTF range, while the other was placed $\sim 1 \mathrm{~km}$ away at the range operation center for comparison of local weather patterns. Additionally, detailed light detection and ranging (LIDAR) data, differential GPS, and ground truth measurements of the building and road dimensions were collected for model geometry creation and georeferencing.

Table ES-1.1.1. OPTUS 1 data collection summary.

\begin{tabular}{lcc}
\hline \multicolumn{1}{c}{ Data Collected } & Report Section & Page Number \\
\hline \hline $\begin{array}{l}\text { HPGE OUTDOOR BUILDING } \\
\text { AND GROUND SURFACE }\end{array}$ & 1.1 & 1 \\
MEASUREMENTS & & \\
NaI(Tl) STATIC & 1.2 & 4 \\
MEASUREMENTS & & \\
NaI(Tl) DYNAMIC & 1.3 & 8 \\
BACKGROUND & & \\
MEASUREMENTS & 1.4 & 10 \\
GEOREFERENCING & 1.5 & \\
LIDAR MAPPING OF CACTF \\
$\begin{array}{l}\text { BUILDING EXTERIORS } \\
\text { METEOROLOGICAL AND }\end{array}$
\end{tabular}

The second data collection, OPTUS 2, was performed at FTIG in August 2015 and was targeted to collect further refined data including those to fill in gaps identified from the analysis of OPTUS 1 data. 
Additional shielded HPGe measurements were performed to evaluate the variation of NORM values for similar materials across the site. Some duplicate measurements were performed with the 2 in. $\times 4$ in. $\times$ 16 in. $\mathrm{NaI}(\mathrm{Tl})$ detectors to correlate with static data from OPTUS 1. Further $\mathrm{NaI}(\mathrm{Tl})$ measurements were performed in the interior of a hotel building with and without a ${ }^{137} \mathrm{Cs}$ source for model validation of the hotel. LIDAR data was taken in the interior of the hotel as well, to accompany the interior $\mathrm{NaI}(\mathrm{Tl})$ data for model reconstruction. From OPTUS 1, it was determined the weather patterns varied from the range operation center to the CACTF. Therefore, the persistent radiation and meteorological monitoring stations were placed on the CACTF range to monitor the radiation response resulting from weather phenomena that occurred near the measurements being performed. In addition, a shielded directional $\mathrm{NaI}(\mathrm{Tl})$ detector was used to determine the relative directional background source term seen by the solid angle of the detector. Neutron measurements were also taken to evaluate the importance of background neutron collection at the site for future radiation transport modeling.

Table ES-1.1.2. OPTUS 2 data collection summary.

\begin{tabular}{lcc}
\hline \multicolumn{1}{c}{ Data Collected } & Report Section & Page Number \\
\hline \hline $\begin{array}{l}\text { HPGe OUTDOOR BUILDING AND } \\
\text { GROUND SURFACE }\end{array}$ & 2.1 & 17 \\
MEASUREMENTS & & \\
NaI(TI) STATIC MEASUREMENTS & 2.2 & 20 \\
NaI(TI) INDOOR & 2.3 & 20 \\
MEASUREMENTS & & \\
DIRECTIONAL NaI(Tl) & 2.4 & 23 \\
BACKGROUND SOURCE TERM & & \\
MEASUREMENTS & 2.5 & 25 \\
STATIC NEUTRON & 2.6 & 26 \\
MEASUREMENTS & & 27 \\
GEOREFERENCING & 2.7 & \\
LIDAR MAPPING OF CACTF & & \\
BUILDING INTERIORS & & \\
METEOROLOGICAL AND & 2.8 & \\
PERSISTENT RADIATION & & \\
MONITORING & &
\end{tabular}

The third data collection, OPTUS 3, was performed at FTIG in November 2015. This data collection was performed solely by Remote Sensing Laboratory and aimed to collect $\mathrm{NaI}(\mathrm{Tl})$ measurements along Main St. at the CACTF using 2 in. $\times 4$ in. $\times 16 \mathrm{NaI}(\mathrm{Tl})$ Radiation Solutions Inc. (RSI) systems. This dataset was used to validate the virtual testbed created using geometry measurements and source terms derived from HPGe measurements taken at OPTUS 1 and 2.

Table ES-1.1.3. OPTUS 3 data collection summary.

\begin{tabular}{ccc}
\hline Data Collected & Report Section & Page Number \\
\hline \hline RSI NaI(Tl) MEASUREMENTS & 3.1 & 30 \\
\hline
\end{tabular}

The final data collection at FTIG, MUSE 1, was performed in September 2016. This data collection aimed to measure benchmark-quality controlled dynamic data from a variety of radiation sources including special nuclear material and various optical imaging measurements for point cloud development. Because of logistical issues, no radiation sources arrived on-site, however measurements 
without sources were still performed. These measurements included additional HPGe measurements to address materials lacking measurements and anomalies in previous data. Static $\mathrm{NaI}(\mathrm{Tl})$ background measurements were performed with the same cart setups as before as well as controlled dynamic measurements with unmanned ground vehicles using lessons learned from OPTUS 1,2 and 3. Additional LIDAR data was taken with a different LIDAR unit for comparison with the previous ground truth measurements. An unmanned aerial vehicle was also equipped with various optical cameras used to collect stills, video, and hyperspectral data to be used for geometry reconstruction. The Radiological Multisensor Analysis Platform (RadMAP) was used to perform static and dynamic measurements across the CACTF site simultaneously and synchronously collecting gamma-ray, neutron, odometry, hyperspectral, LIDAR and video data. In addition to the OPTUS and MUSE measurements, additional measurements were performed with multiple backpack platforms that are being developed through other agencies (Domestic Nuclear Detection Office [DNDO]/Wearable Intelligent Nuclear Detection [WIND], Defense Threat Reduction Agency [DTRA]/ Enhanced Mapping System [EMAPS], and AIPT/NA84).

Table ES-1.1.4. MUSE 1 data collection summary.

\begin{tabular}{lcc}
\hline \multicolumn{1}{c}{ Data collected } & Report section & Page number \\
\hline \hline $\begin{array}{l}\text { HPGe OUTDOOR BUILDING AND } \\
\text { GROUND SURFACE }\end{array}$ & 4.1 & 45 \\
MEASUREMENTS & & \\
NaI(TI) STATIC BACKGROUND & 4.2 & 50 \\
MEASUREMENTS & & \\
NaI(TI) DYNAMIC & 4.3 & 51 \\
BACKGROUND & & 55 \\
MEASUREMENTS & 4.4 & 55 \\
GEOREFERENCING & 4.5 & \\
LIDAR/VISUAL MAPPING OF & & 58 \\
CACTF BUILDING EXTERIORS & 4.6 & 63 \\
METEOROLOGICAL AND & & 104 \\
PERSISTENT RADIATION & 4.7 & \\
MONITORING & & \\
RadMAP MEASUREMENTS & 4.8 & \\
WIND | EMAPS | AIPT & & \\
MEASUREMENTS & &
\end{tabular}

\section{MEASUREMENT IMPACT ON VIRTUAL TESTBED}

HPGe background source term measurements of material at FTIG (cinderblock walls, dirt, asphalt, concrete, etc.) taken in OPTUS 1, OPTUS 2 and MUSE 1 were used to create a catalog of potassiumuranium-thorium concentrations and uncertainties. This catalog has been used to inform background source terms in a large-scale particle transport model of the FTIG facility, which has been used to calculate detector response functions for 2 in. $\times 4$ in. $\times 16$ in. $\mathrm{NaI}(\mathrm{Tl})$ detectors along Main St. See Section 4.1 for a more detailed discussion.

Calculated 2 in. $\times 4$ in. $\times 16$ in. $\mathrm{NaI}(\mathrm{Tl})$ detector response functions have been validated against static measurements taken along Main St. in OPTUS 3 (Section 3.2). The calculated response deviates the highest at low energies because of the absence of skyshine transport in the preliminary FTIG model. Skyshine transport is being added to the FTIG virtual testbed to bring calculated detector response 
functions closer to validation measurements. This work will also study the physics principles behind skyshine's effect on the detector response.

The particle transport testbed can produce synthetic time series data for radiation detection algorithm evaluation. The static and dynamic 2 in. $\times 4$ in. $\times 16$ in. NaI(Tl) measurements made in MUSE 1 provide validation for the synthetic data generation methodology. Time series synthetic data, generated by interpolating between nearest neighbor static measurements and Poisson sampling, were compared with dynamic measurements (Section 4.3). These benchmark measurements were used to validate a synthetic data generation methodology for the upcoming data competition.

Visual photography, thermal images, and LIDAR data were taken using a variety of technologies and modalities including static high-quality measurements, terrestrial robot, vehicle, pedestrian, and unmanned aerial vehicles. Point clouds have been generated from these measurement technologies and compared for accuracy. These point clouds can and will be used to generate computer-aided design models for fast particle transport model development. See Sections 1.4, 1.5, 2.7, 4.5 and 4.7 for more information.

\section{FUTURE WORK}

The collaboration will perform sensitivity studies using the FTIG model to understand important model parameters on detector response and radiation detection algorithm performance. This will include varying model geometries and materials. Changes will be quantified through parsing synthetic datasets through benchmark algorithms such as those that perform well in the upcoming data competition.

Measurements at the FTIG facility were invaluable to validate particle transport and synthetic data generation methodologies for a virtual testbed of the FTIG facility. New measurements at other sites, including both source term generation using HPGe measurements and $\mathrm{NaI}(\mathrm{Tl})$ model validation, are needed to understand what is needed to model new background environments. These new measurements will be added to the catalog of background source terms generated from FTIG to better understand the variation of potassium-uranium-thorium concentrations from environment to environment.

Skyshine will be added to the particle transport testbed to better match detector response calculations with the low energy portion validation measurements. In this study, systematic variations in the skyshine component to the response function will be investigated along with correlations to nearby material, such as buildings and far field geometries. Further measurements in new environments, along with new particle transport models, are needed to study these systematic variations.

The effect of clutter - objects that may enter or exit an environment (e.g., vehicles, people, etc.) —on detector responses and algorithm performance is not well known. Further static measurements will be taken in a new environment with and without simplified versions of clutter to validate particle transport models. These simplified clutter versions may be stacked buckets of water to approximate a human being or materials of varied densities to simulate other possible surroundings. Using these models, the suppression of background by clutter will be quantified to determine which components of the background model geometry are most suppressed. In addition, the suppression caused by simplified clutter will be compared to more realistic versions of clutter (e.g., understand how close the attenuation effects of a real person are to those of stacked buckets of water). The results of this study will inform systematic uncertainties in calculated detector response functions due to clutter.

New research on including contextual sensors (e.g., LIDAR, video, thermal imagery, etc.) is a trending topic in the radiation detection community. Having the ability to simulate imagery from these devices, tied to synthetic radiation detection datasets, will be valuable in designing and evaluating new radiation 
detection systems. The generation of synthetic contextual sensor data will need to be validated against measurements in well-controlled environments followed by environments with some dynamic behavior (such as moving clutter and radiation sources) and purely uncontrolled datasets that have been obtained by RadMAP. New benchmark measurements using a combination of contextual sensors and radiation detectors will be made in a new environment for model validation.

There will be ongoing analysis of the data generated by the RadMAP system at FTIG. The analyses include: effort to quantify the precision and accuracy to which RadMAP is able to determine spectral emission rates from surfaces observed by one or more pass-bys; quantifying means of analyzing hyperspectral imagery data collected from ground-based systems; and using RadMAP sensors to automatically identify clutter within measured data and to define how that clutter impacts observations.

Finally, neutron transport will be added to the particle transport testbed, including cosmic-ray background source terms. The background and source detector response calculations will need to be validated against benchmark measurements in realistic urban scenarios with tall buildings. It has been shown that the angle of open sky in an urban environment can suppress the measured neutron background by as much as $30 \%$ [1]. In addition, the effect of clutter and spallation events caused by high z-material near the detector will be investigated.

\section{LESSONS LEARNED}

1) A more accessible static measurement site is needed. More frequent, smaller measurement campaigns with greater site flexibility do not require a major (expensive) equipment move and allows for data to be recaptured as needed to fill holes in the dataset.

2) A second measurement site to provide data to contribute to the background modeling is also needed. For instance, does the background model at FTIG work to build a representative background somewhere else?

3) A soil moisture sensor should be included with the weather system while collecting radiation-weather correlation data. After a heavy rain the background may become suppressed because of shielding from water-logged soil.

4) To compare similar measurements, the data collection geometries need to be as similar as possible. For example, there are systematic differences between measurements made with a 2 in. $\times 4$ in. $\times$ 16 in. $\mathrm{NaI}(\mathrm{Tl})$ detector in a low-scatter cart and in a large vehicle.

5) Having a detailed understanding of nonproportionality light production in the detector response for all fielded detectors is very important when comparing detector response calculations. A careful and detailed procedure was developed after OPTUS 1, and the procedure employed in other measurement campaigns.

6) A benchmark set of laboratory measurements is needed for comparison purposes after software updates are made to a data acquisition system. This was done after OPTUS 2 to prevent future data acquisition errors.

7) Skyshine is an important component of the low energy portion of measured unshielded detector response functions. This effect needs to be included when comparing calculated and measured detector response functions.

8) The error associated with GPS location data is too large to be used for benchmark dynamic radiation detector measurements. Instead, ground truth measurements (tape measure and stop watch) or a 
system capable of starting and stopping from well-known positions while moving at a constant speed are needed with careful documentation.

9) RadMAP's deployment resulted in an unanticipated issue due to lack of connectivity while deployed. RadMAP uses a network time protocol (NTP) server to maintain synchronization across the acquisition systems; however, it was observed that the NTP server exhibited greater time drift because it was only connected to GPS as the sole time source. The team observed that when multiple time sources are provided to NTP (e.g., GPS and a NIST.gov time server) the on-board NTP server remained stable to a few milliseconds resulting in $\sim 200$ millisecond drift, relative to GPS was observed, but logged.

10) GPS base stations in the vicinity of FTIG are greater distances from the system than the previous RadMAP in the San Francisco Bay Area. Higher frequency course over-ground message logging would likely have improved the performance of the INS-based localization solutions. 



\section{OPTUS 1 MEASUREMENT CAMPAIGN}

The first data collection campaign, OPTUS 1, was performed in May 2015 at the Combined Arms Collective Training Facility (CACTF) in Fort Indiantown Gap (FTIG) and aimed to collect all details necessary for model creation including radiation data, geometries, and metadata as accurately as possible. Sample background radiation levels for a variety of materials present at the site (including cinder block, gravel, asphalt, and soil) were collected using shielded long dwell high-purity germanium (HPGe) measurements to quantify the contributing sources of naturally occurring radioactive materials (NORM). In addition to the shielded HPGe measurements, benchmark quality unshielded 2 in. $\times 4$ in. $\times 16$ in. $\mathrm{NaI}(\mathrm{Tl})$ data were taken at a variety of locations in the FTIG site to validate model output. On-site persistent radiation and meteorological monitoring stations were used to monitor the radiation response of potential weather phenomena. One station was placed on the CACTF range, and the other was placed at the range operation center (ROC) for comparison of local weather patterns. Additionally, detailed light detection and ranging (LIDAR) data, differential global positioning system (GPS), and ground truth measurements were collected for model geometry creation.

\subsection{HPGE OUTDOOR BUILDING AND GROUND SURFACE MEASUREMENTS}

To characterize the concentration of NORM in the various materials found at the FTIG facility, a series of 36 shielded HPGe measurements were performed. In these experiments, a mechanically cooled ORTEC EX-100T HPGe detector was placed against soil, asphalt roads, gravel lots, concrete intersections, sidewalks, building foundations, and walls throughout the site. Once the detector was encased in a lead sarcophagus to minimize gamma-ray interactions from other materials, a spectrum was recorded for approximately $30 \mathrm{~min}$. A comprehensive list of all 36 measurements can be seen in Table 1.1.1, including brief descriptions of each measurement and the file name of the recorded spectra. A photograph of one measurement is shown in the upper left corner of Figure 1.1.1.

Table 1.1.1. Comprehensive list of OPTUS 1 HPGe measurements.

\begin{tabular}{|c|c|c|c|}
\hline ID & Material & Description & File name \\
\hline H01 & Concrete patio & 1-story shop (bar) on Main St. side & H1-1327-15May2015-barPorchMainStreetSide \\
\hline H02 & Soil & 1-story shop (bar) on Main St. side & H2-1410-15May2015-BarGrass \\
\hline $\mathrm{H} 03$ & $\begin{array}{l}\text { Concrete } \\
\text { sidewalk }\end{array}$ & 1-story shop (bar) on Main St. side & H3-1455-15May2015-BarSidewalk \\
\hline H04 & Asphalt & 1-story shop (bar) on Main St. side & H4-1548-15May2015-BarAsphalt \\
\hline H05 & $\begin{array}{l}\text { Concrete, } \\
\text { intersection }\end{array}$ & $\begin{array}{l}\text { Intersection of Main and Church, near } \\
\text { bar }\end{array}$ & H5-1703-15May2015BarConcreteRoad \\
\hline H06 & Concrete, patio & $\begin{array}{l}\text { 1-story shop (bar), facing int. of Main } \\
\text { and Church }\end{array}$ & H6-1750-15May2015-BarPorch \\
\hline H07 & Concrete, patio & 1-story shop (bar) on Church St. side & H7-1345-16Мay2015-BarPorch \\
\hline H08 & Soil & 1-story shop (bar) on Church St. side & H8-1435-16May2015-BarGround \\
\hline $\mathrm{H} 09^{*}$ & $\begin{array}{l}\text { Concrete, } \\
\text { sidewalk }\end{array}$ & 1-story shop (bar) on Church St. side & H9-1002-17May2015-BarSidewalk \\
\hline $\mathrm{H} 10^{*}$ & Asphalt & Main St., in front of 2-story office & H10-1112-17May2015-OfficeAsphalt \\
\hline $\mathrm{H} 11^{*}$ & Block, light tan & 2-story office, side facing 1-story shop & H11-1309-17May15-OfficeWall \\
\hline $\mathrm{H} 12 *$ & Gravel & Service station, $0.75-1$ in. thick & $\begin{array}{l}\text { H12-1420-17May2015- } \\
\text { ServiceStationGravelPoint }\end{array}$ \\
\hline H13 & Block, white & Service station, Main St. side, on mortar & $\begin{array}{l}\text { H13-1530-17May2015-ServiceStationWall- } \\
\text { MorterPoint2 }\end{array}$ \\
\hline
\end{tabular}


Table 1.1.1. Comprehensive list of OPTUS 1 HPGe measurements (continued).

\begin{tabular}{|c|c|c|c|}
\hline ID & Material & Description & File Name \\
\hline H14 & Block, white & $\begin{array}{l}\text { Service station, Main St. side, not on } \\
\text { mortar }\end{array}$ & $\begin{array}{l}\text { H14-1610-17May2015-ServiceStationWall- } \\
\text { VoidPoint3 }\end{array}$ \\
\hline H15 & Soil & Service station, Main St. side & $\begin{array}{l}\text { H15-1655-17May2015- } \\
\text { ServiceStationGroundPoint4 }\end{array}$ \\
\hline H16 & $\begin{array}{l}\text { Concrete, } \\
\text { sidewalk }\end{array}$ & Service station, Main St. side & $\begin{array}{l}\text { H16-1744-17May2015- } \\
\text { ServiceStationSidewalkPoint5 }\end{array}$ \\
\hline $\mathrm{H} 17 *$ & Gravel & $\begin{array}{l}\text { Between 2-story office and 1-story shop, } \\
3 \text { in. thick }\end{array}$ & H17-1834-17May2015-OfficeGravel \\
\hline $\mathrm{H} 18^{*}$ & Soil & 2-story office, side facing 1-story shop & H18-944-18May2015-OfficeGroundPoint \\
\hline H19 & Gravel & Between service station and OPS/storage & H19-1036-18May2015-ShedGravelPoint \\
\hline $\mathrm{H} 20^{*}$ & Block, dark tan & $\begin{array}{l}\text { OPS/storage building, service station } \\
\text { side }\end{array}$ & H20-1139-18May2015-ShedWalPoint \\
\hline $\mathrm{H} 21 *$ & $\begin{array}{l}\text { Concrete, } \\
\text { sidewalk }\end{array}$ & Between OPS/Storage and dumpster area & $\begin{array}{l}\text { H21-1308-18May2015- } \\
\text { DumpsterSidewalkPoint }\end{array}$ \\
\hline $\mathrm{H} 22$ & Soil & Dumpster area & H22-1406-18May2015-DumpsterGroundPoint \\
\hline $\mathrm{H} 23 *$ & Gravel & $\begin{array}{l}\text { Dumpster area, where sidewalk should } \\
\text { be }\end{array}$ & H23-1523-18May2015-DumpsterGravelPoint \\
\hline $\mathrm{H} 24 *$ & $\begin{array}{l}\text { Concrete, } \\
\text { sidewalk }\end{array}$ & Police station, in front of main stairs & $\begin{array}{l}\text { H24-1709-18May2015- } \\
\text { PoliceStationSidewalk1 }\end{array}$ \\
\hline $\mathrm{H} 25^{*}$ & $\begin{array}{l}\text { Concrete, } \\
\text { sidewalk }\end{array}$ & 3-story hotel, in front of main stairs & H25-1622-18May2015-HotelSidewalkPoint1 \\
\hline $\mathrm{H} 26$ & Concrete, patio & 3 -story hotel & H26-1008-19May2015-HotelStepsPoint2 \\
\hline $\mathrm{H} 27$ & $\begin{array}{l}\text { Concrete, } \\
\text { foundation }\end{array}$ & 3-story hotel & $\begin{array}{l}\text { H27-1050-19May2015- } \\
\text { HotelCementFoundationPoint } 3\end{array}$ \\
\hline $\mathrm{H} 28 *$ & Block, grey & $\begin{array}{l}\text { 3-story hotel, wall on front porch, facing } \\
\text { inwards }\end{array}$ & $\begin{array}{l}\text { H28-1205-19May2015-HotelInnerWallPorch- } \\
\text { GreyPoint4 }\end{array}$ \\
\hline $\mathrm{H} 29 *$ & $\begin{array}{l}\text { Concrete, } \\
\text { intersection }\end{array}$ & $\begin{array}{l}\text { Intersection of Main and Maple, near } \\
\text { police station }\end{array}$ & $\begin{array}{l}\text { H29-1426-19May2015- } \\
\text { PoliceStationCementRoadPoint } 2\end{array}$ \\
\hline H30 & Asphalt & Police station, in front of main stairs & $\begin{array}{l}\text { H30-1552-19May2015- } \\
\text { PoliceStationAsphaltPoint3 }\end{array}$ \\
\hline $\mathrm{H} 31^{* *}$ & Concrete, curb & $\begin{array}{l}\text { Police station, in front of main stairs } \\
\text { (some asphalt under detector) }\end{array}$ & $\begin{array}{l}\text { H31-1512-19May2015- } \\
\text { PoliceStationCurbPoint4 }\end{array}$ \\
\hline $\mathrm{H} 32 * *$ & Block, red & $\begin{array}{l}\text { Police station rubble wall, facing 2-story } \\
\text { office (Cs source in use) }\end{array}$ & $\begin{array}{l}\text { H32-1806-19May2015- } \\
\text { PoliceStationWall12min } \backslash \text { H32a-1838- } \\
\text { 19May2015-PoliceStationWallPoint5-Part2 }\end{array}$ \\
\hline H33 & Block, light tan & 2-story office rubble wall, cemetery side & H33-920-20May2015-OfficeRubbleWallPoint5 \\
\hline $\mathrm{H} 34 * *$ & Block, light tan & $\begin{array}{l}\text { 2-story office rubble wall, cemetery side, } \\
\text { no lead shielding }\end{array}$ & H34-1007-17May2015-OfficeWall-unshielded \\
\hline H35 & $\begin{array}{l}\text { Concrete, } \\
\text { foundation }\end{array}$ & 2-story office & $\begin{array}{l}\text { H35-1058-20May2015- } \\
\text { OfficeFoundationPoint } 7\end{array}$ \\
\hline $\mathrm{H} 36^{* *}$ & $\begin{array}{l}\text { Concrete, } \\
\text { intersection }\end{array}$ & $\begin{array}{l}\text { Intersection of Main and Maple, near } \\
\text { police station }\end{array}$ & $\begin{array}{l}\text { H36-1151-20May2015- } \\
\text { PoliceStationCementRoadredoPoint }\end{array}$ \\
\hline
\end{tabular}

* Rejected measurements due to HPGe overheating

** Rejected measurements due to other reasons for error 

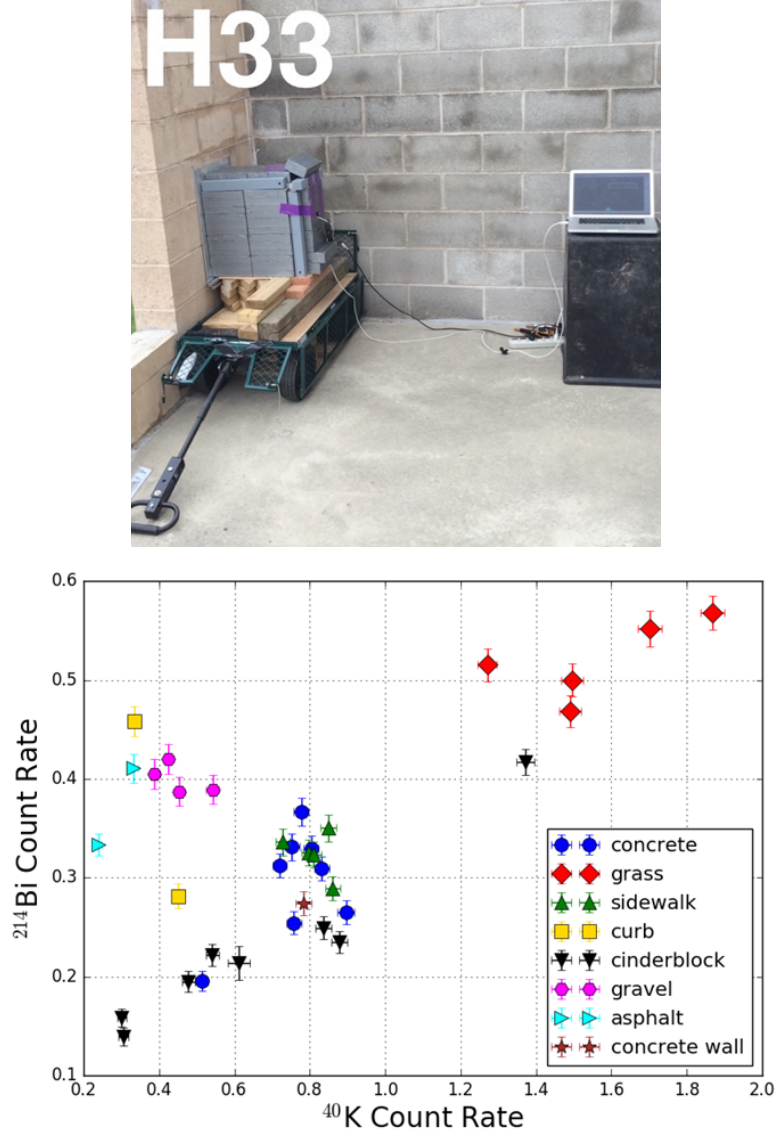
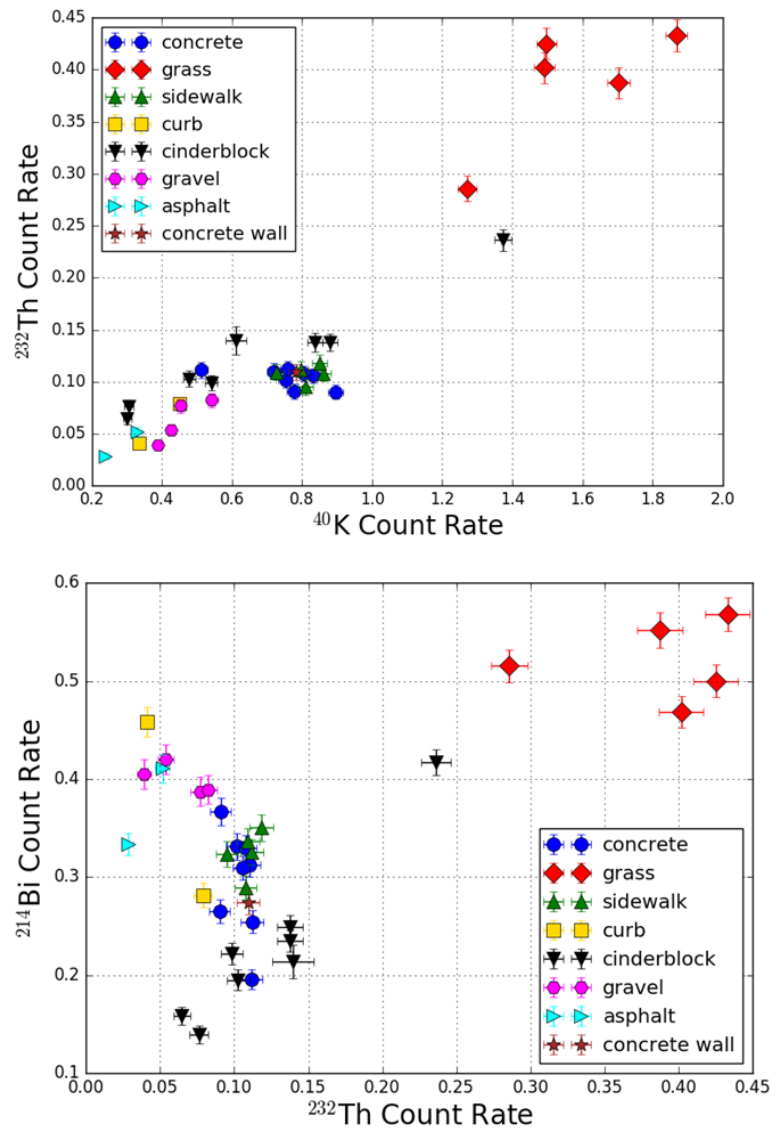

Figure 1.1.1: (Top left) Photograph of a HPGe measurement. The detector is facing the cinder block wall. (Top right) Correlation between $1,460 \mathrm{keV}{ }^{40} \mathrm{~K}$ and $2,614 \mathrm{keV}{ }^{232} \mathrm{Th}\left({ }^{208} \mathrm{Tl}\right)$ peak areas for the OPTUS 1 Measurement Campaign. (Bottom left) Correlation between $1,460 \mathrm{keV}{ }^{40 \mathrm{~K}}$ and $609 \mathrm{keV}^{214} \mathrm{Bi}$ peak areas. (Bottom right) Correlation between $2,614 \mathrm{keV}{ }^{232} \mathrm{Th}\left({ }^{208} \mathrm{Tl}\right)$ and $609 \mathrm{keV}{ }^{214} \mathrm{Bi}$ peak areas.

Nineteen of the 36 measurements taken at the first OPTUS campaign were used in the final set of analysis to determine the concentration of NORM in the various materials around the FTIG site. The measurements marked with an asterisk in Table 1.1 .1 were rejected by the scientists taking or evaluating the data. This was partly because of suspicions that the detector was overheating. This lesson learned prompted the acquisition of a cooling system for the detector throughout the day's measurements. Four other measurements were not used for different reasons upon further analysis of the data. These measurements and reasoning for their rejection are as follows: H31 (unclear measurement surface), H32 (Cs source in use), H34 (no lead shielding), and H36 (determined to be an outlier).

NORM count rates for ${ }^{214} \mathrm{Bi},{ }^{40} \mathrm{~K}$, and ${ }^{232} \mathrm{Th}\left({ }^{208} \mathrm{Tl}\right)$ extracted from the HPGe spectra are also shown in Figure 1.1.1, in the top right, bottom left, and bottom right areas of the figure. One can quickly see that there is clustering in NORM count rates measured over the materials measured. The cinderblock outlier was the measurement taken without lead shielding. From this analysis, one can see that the NORM constituents of similar materials have similar concentrations. This result is to be expected as the entire FTIG CACTF facility was built at one time, with materials coming from similar sources. In a more realistic urban environment, more variation in the NORM concentrations is expected because cities are built over time using materials sourced from a variety of locations and manufactures. 
These measurements add to our understanding of the variation of NORM concentrations at FTIG and can be used to inform systematic studies in detector response affected by variations in NORM concentrations in FTIG models. In addition, these measurements were used to inform concentrations for other models, including Mean Street and Chameleon Street, used to generate data for the upcoming data competition. An in-depth discussion of the analysis of the data gathered from the measurements conducted as part of OPTUS 1 is presented later in this section of the report, along with the results from OPTUS 2 and MUSE 1.

\subsection{NaI(TI) STATIC MEASUREMENTS}

In OPTUS 1, 160 static 10 min. gamma-ray background measurements were taken along Main St. in the FTIG MOUT facility. These measurements were performed to provide data to validate particle transport models, to measure background variability in fine detail along one portion of the FTIG MUOT, and to provide data to test and develop synthetic data generation. A photograph of a low-mass cart housing a 2 in. $\times 4$ in. $\times 16$ in. $\mathrm{NaI}(\mathrm{Tl})$ detector, data acquisition system, and GPS is shown in Figure 1.2.1 along with the measurement locations. The red arrow denotes the order of measurements displayed in the lower portion of Figure 1.2.1.
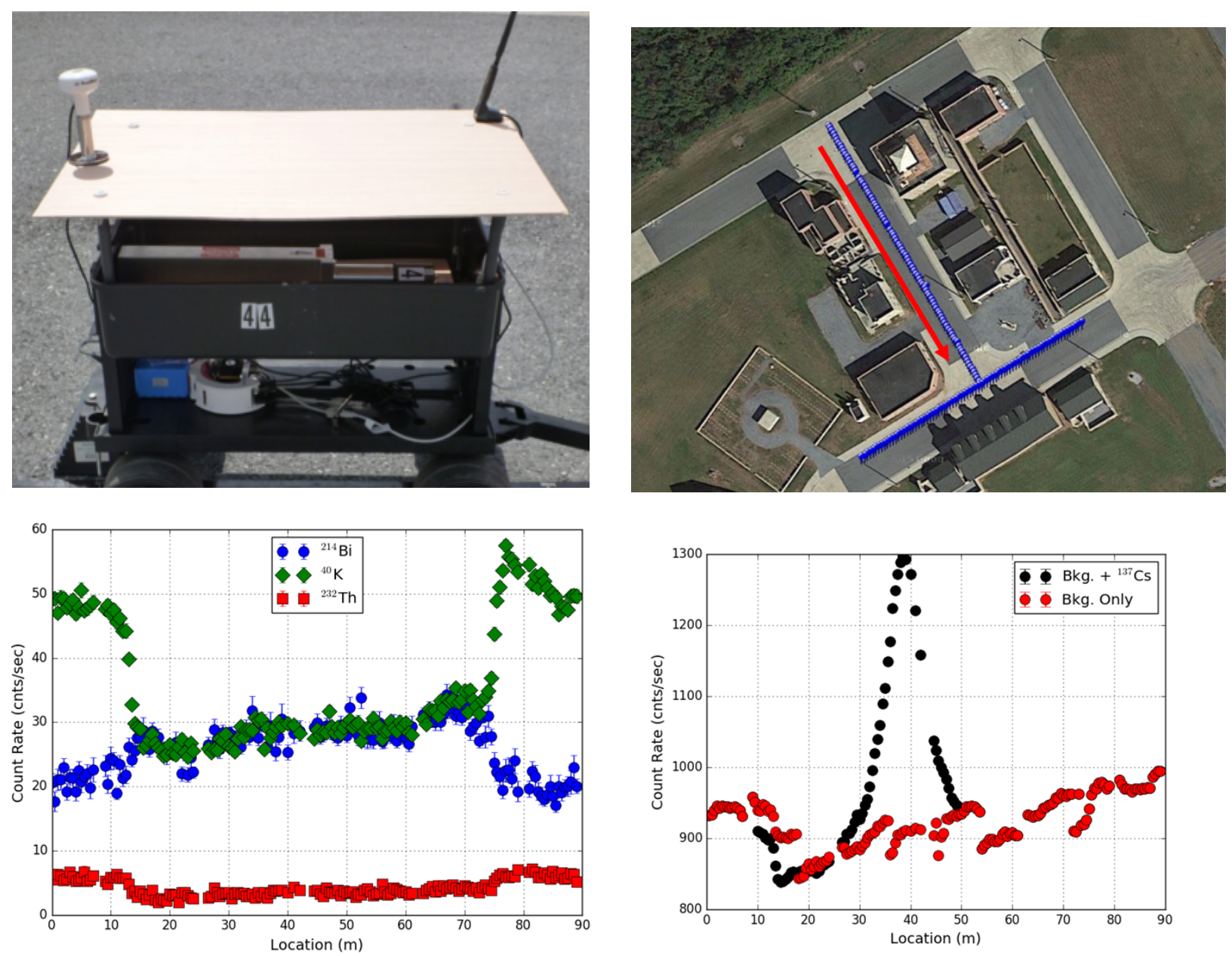

Figure 1.2.1. OPTUS 1 static measurement results. (Top left) Photograph of NaI(TI) detector in a low scatter cart. (Top right) Static positions of 2 in. $\times 4$ in. $\times 16$ in. NaI(TI) detector measurements down Main St. The distance between nearest neighbor positions is $0.5 \mathrm{~m}$. (Bottom left) $609 \mathrm{keV}{ }^{214} \mathrm{Bi}, 1460 \mathrm{keV}{ }^{40} \mathrm{~K}$, and $2614 \mathrm{keV}{ }^{232} \mathrm{Th}$ peak areas as a function of path down Main St. (Bottom right) Background (red circles) and background and ${ }^{137}$ Cs (black circles) total count rates down Main St. in the FTIG MUOT. 
The variation in the gamma-ray background at FTIG was found to be fairly small, as can be seen in the bottom two figures of Figure 1.2.1. All measured gamma-ray spectra were energy calibrated using the ${ }^{214} \mathrm{Bi},{ }^{40} \mathrm{~K}$, and ${ }^{232} \mathrm{Th}\left({ }^{208} \mathrm{Tl}\right) \mathrm{NORM}$ peaks and placed in a uniform 1,024 binning structure. The ${ }^{40} \mathrm{~K}$ peak area was smallest in measurements made along the asphalt road ( $\sim 30$ counts per second [cps]), but increased when measured on the concrete portions of FTIG ( $\sim 50 \mathrm{cps})$. Similarly, the ${ }^{214} \mathrm{Bi}$ and ${ }^{232} \mathrm{Th}$ peak areas were consistent along the asphalt road and changed when measured on the concrete portions of FTIG. In addition to the background measurements, data with a ${ }^{137} \mathrm{Cs}$ source were taken along the same path. The resulting total count rates can be seen in the lower right quadrant of Figure 1.2.1.

Unfortunately, there was no gain stabilization algorithm running for the static 2 in. $\times 4$ in. $\times 16$ in. $\mathrm{NaI}(\mathrm{Tl})$ measurements in OPTUS 1. This accounts for some of the random behavior between nearest neighbor total count rates in background measurements in the lower right corner of Figure 1.2.1. Energy calibration was applied after the measurements were taken based on the ${ }^{214} \mathrm{Bi},{ }^{40} \mathrm{~K}$, and ${ }^{232} \mathrm{Th}$ background peaks using a second order polynomial. This approximation fails in the lower energy portion of the spectrum, because of nonlinearity in $\mathrm{NaI}(\mathrm{Tl})$ crystals. To do a better job in the future, a new calibration procedure was developed by measuring the gain and nonproportionality response over a range of temperatures using a temperature chamber. This information was used to provide better energy calibrations and gain stabilization. Because of calibration differences and gain drift, all comparisons between measurements made in OPTUS 1 and subsequent measurements will divide by the total energy window of each count rate.

These static measurements were used to create synthetic dynamic datasets which can be used for radiation detection algorithm evaluation. The methodology for creating these datasets is described below. First, for a detector speed, $s$, and integration time, $\Delta t$, a uniform set of synthetic detector locations is created in the area where measurements were taken. The number of synthetic detector locations, $N$, is

$$
N=s \Delta t / \Delta d,
$$

where $\Delta d$ is the distance of the synthetic dataset path. Detector responses are assigned to each location by interpolating nearest neighbor detector response measurements. It is important that the change in background or the background plus source detector response functions are changing slowly between nearest neighbor measurements used for interpolation. A schematic for the methodology is shown in Figure 1.2.2 and a resulting interpolated total count rate for a detector moving $8 \mathrm{~m} / \mathrm{s}$ is shown in Figure 1.2.3. 


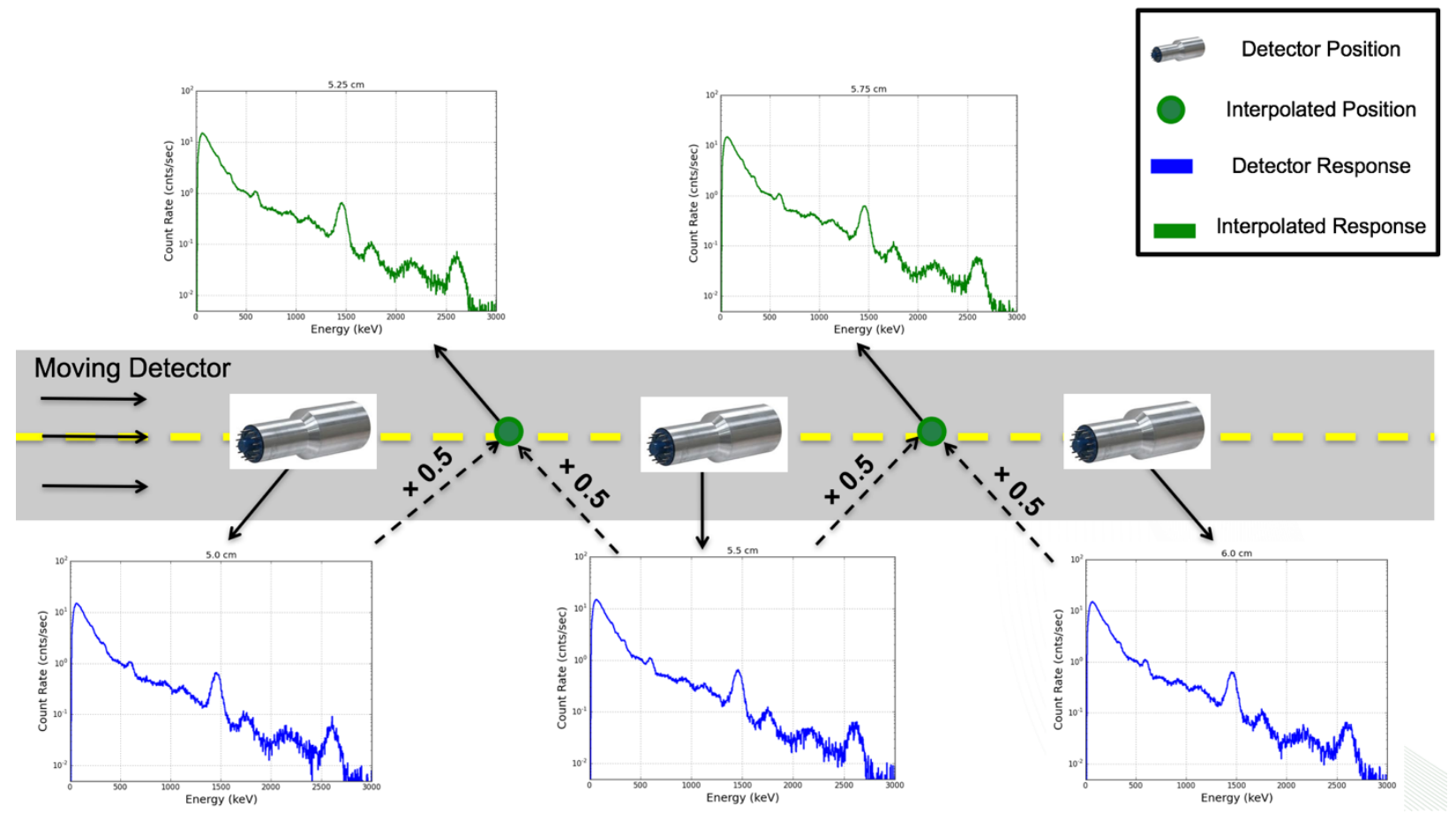

Figure 1.2.2. Illustration of mobile detection methodology using static measurements. (Above) Interpolation procedure used to generate synthetic data from static measurements. (Below) spectra (blue) denote measured detector responses and top spectra (green) denote interpolated detector responses. Interpolation is based on a simple distance weighted average.

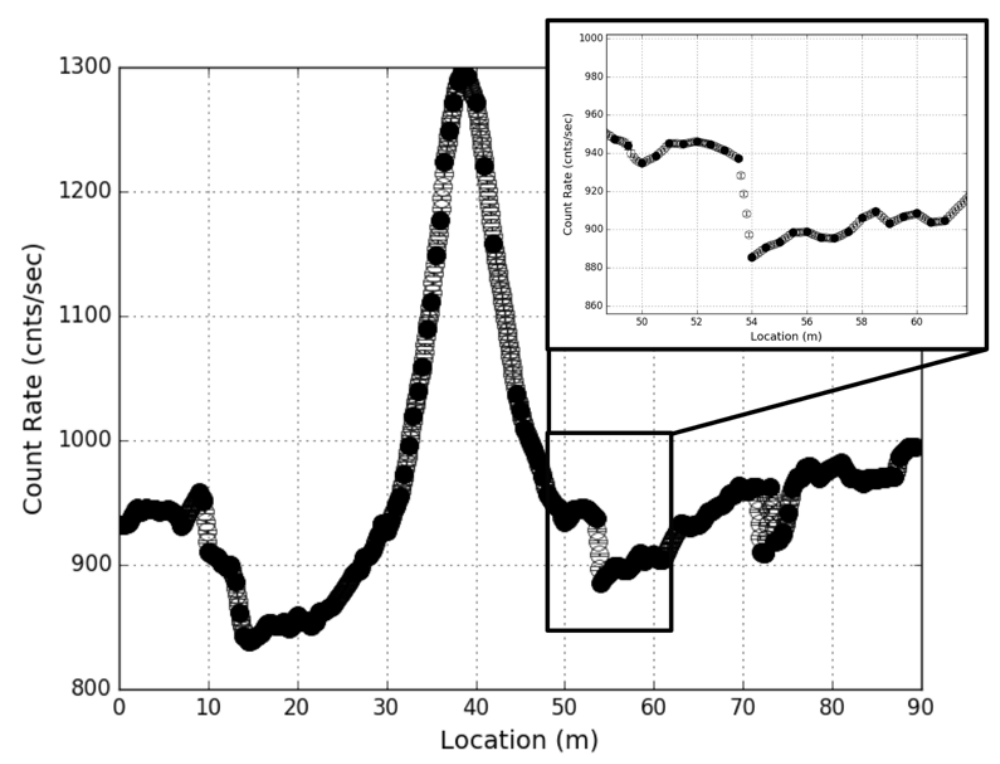

Figure 1.2.3. Example of detector response interpolation. Solid markers denote measured total count rates, and empty markers show interpolated spectra. Change in count rate from $53.5 \mathrm{~m}$ to $54 \mathrm{~m}$ is due to measurements being performed with different detector units. 
Next, the interpolated detector response calculations are down sampled, using Poisson statistics, to generate random noise in the synthetic data. Each interpolated detector response is scaled by the detector integration time and each interpolated detector response channel is sampled. See Figure 1.2.4 for an example detector response function, the Poisson distribution in one channel, and a sample of the entire spectrum for a $0.5 \mathrm{~s}$ integration time. To converge correctly in environments with large background variations, a small detector integration time and short distances between measurement locations is needed so that interpolated detector response functions are smaller than spatial variations in the background. This methodology has been benchmarked against list mode laboratory measurements, and the sampled spectrum converges on the original spectrum for long integration times (Figure 1.2.5).
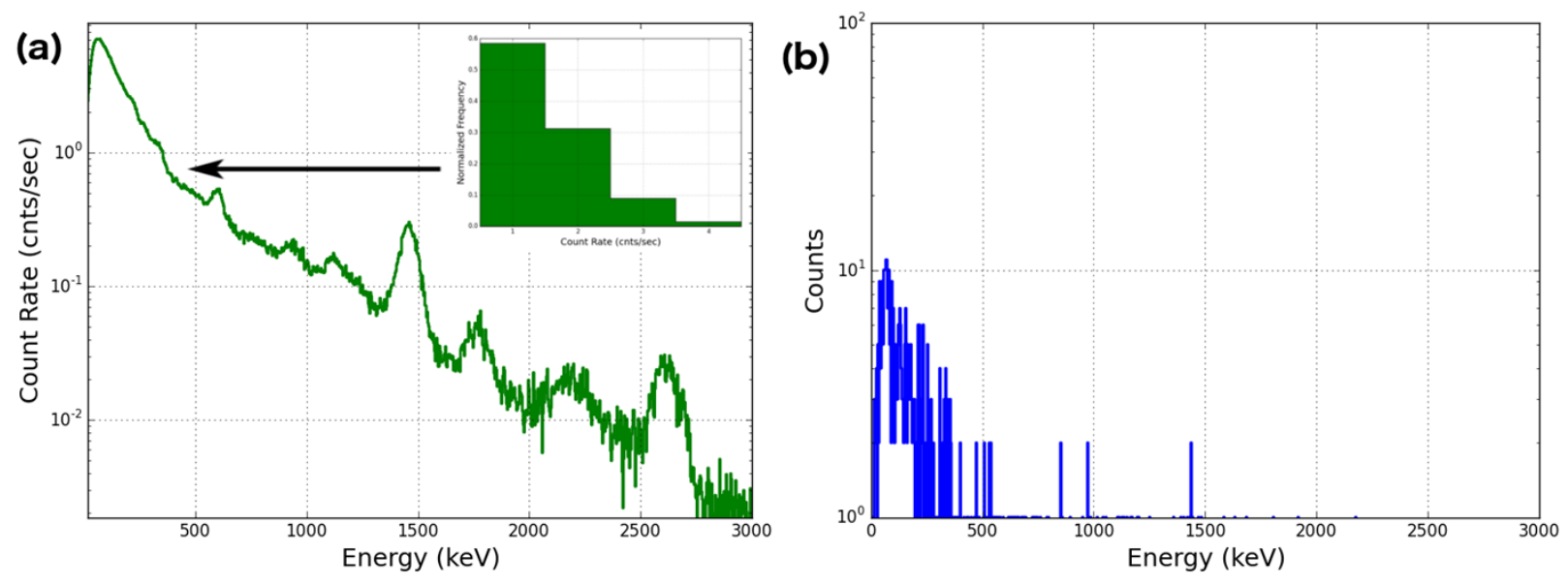

Figure 1.2.4. Sampling procedure used to generate synthetic data. (Left) An interpolated detector response. The inset shows a Poisson distribution of a channel in the detector. (Right) A sample for a detector integration time of $0.5 \mathrm{~s}$.

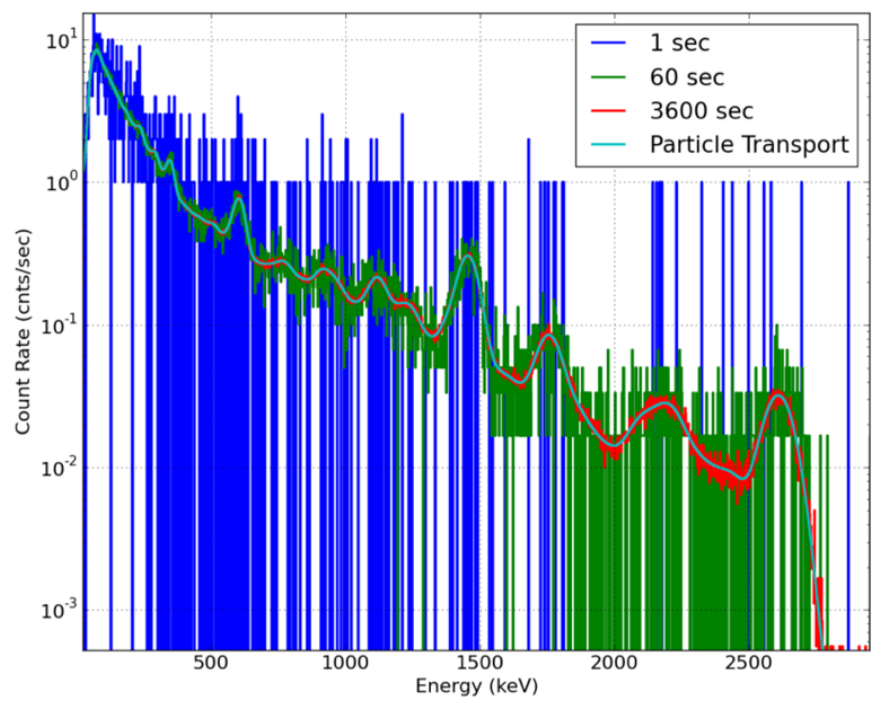

Figure 1.2.5. Example of detector response sampling. Cyan curve shows the original detector response (one taken over infinite time). The red curve represents a detector response for a $1 \mathrm{~h}$ integration time, the green for $1 \mathrm{~min}$. and the blue for $1 \mathrm{~s}$. 
To validate this methodology, multiple dynamic measurements are needed along the same path of the synthetic datasets. These measurements require high precision telemetry to make a good comparison with the synthetic datasets, which is near-impossible with traditional GPS units. This dynamic data was not collected in OPTUS 1 measurement campaign, but was in MUSE 1.

\subsection{NaI(TI) DYNAMIC BACKGROUND MEASUREMENTS}

In OPTUS 1, dynamic background measurements were collected using a 2 in. $\times 4$ in. $\times 16$ in. $\mathrm{NaI}(\mathrm{Tl})$ detector and GPS unit in a van moving at 5, 10 and $20 \mathrm{mph}$ in loops around the CACTF. Sample GPS data and total count rates are shown in the top portion of Figure 1.3.1. Although the GPS data in the van measurements are fairly consistent, there is still some uncertainty (on the order of a meter or so) in detector location resulting from inherent GPS error. The count rates measured in the dynamic van runs were significantly lower than the static measurements made in the detector cart. The average count rate for the series shown in the upper portion of Figure 1.3 .1 is $673.24 \mathrm{cps}$, and the average of all the static cart measurements were $924.9 \mathrm{cps}$, roughly a $37 \%$ decrease. The reason for the lower count rates may be a result of the shielding from the van itself and other material surrounding the detector. Because of this significant difference in detector response exists, and absolute position is not known, these data are not suitable to validate synthetic data generation methodologies, but may be useful to bound expected count rates in particle transport models containing a radiation detector inside of a vehicle.
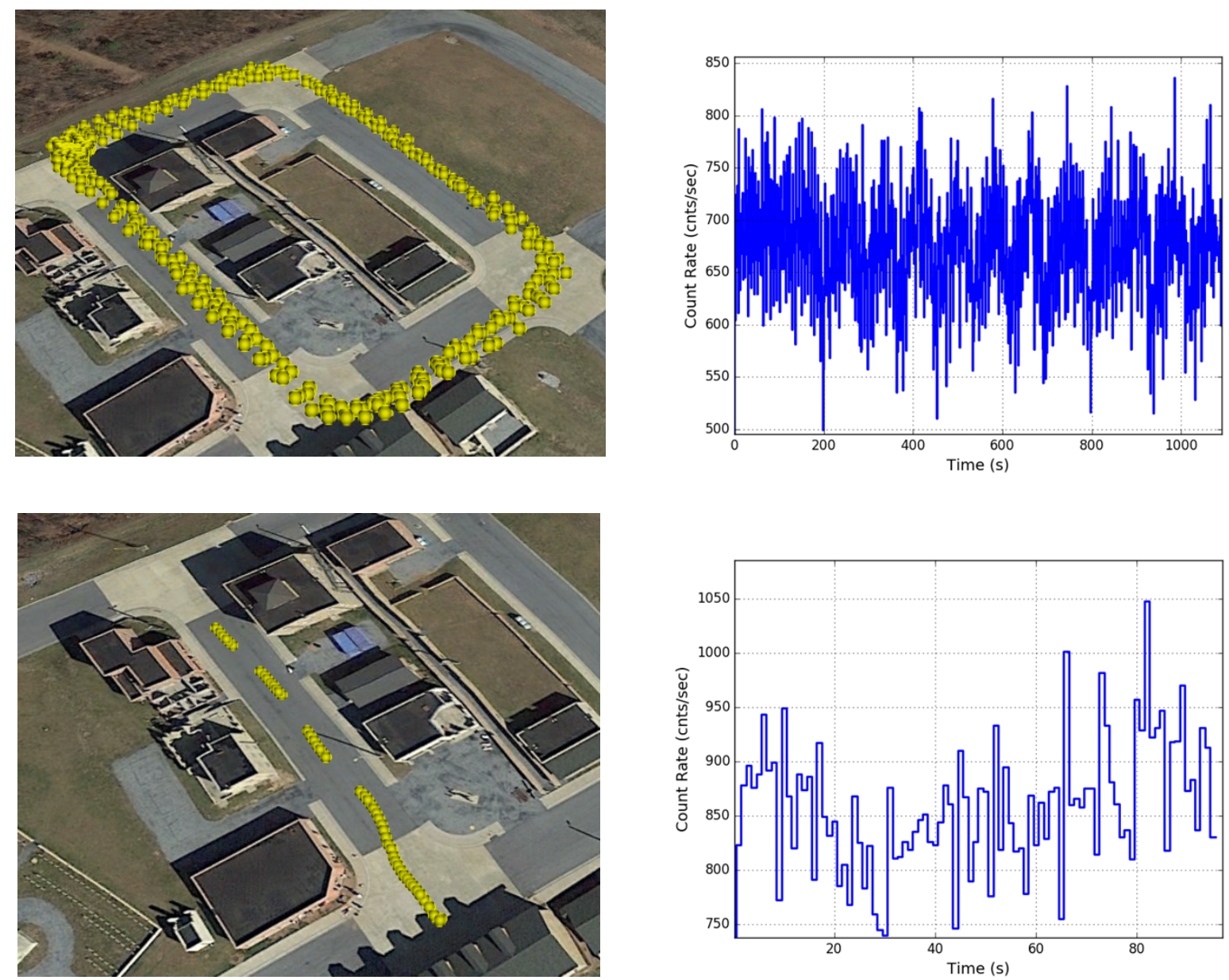

Figure 1.3.1. OPTUS 1 dynamic NaI(TI) measurements at CACTF. (Top left) Van GPS locations for three loops around the CACTF. (Top right) Van total count rates for the three passes shown in the top left. (Bottom 


\section{left) Walking GPS locations for one pass along Main St. (Bottom right) Total count rates for the pass shown in}

the bottom left figure.

In addition to dynamic measurements in a vehicle, a detector cart, again using a 2 in. $\times 4$ in. $\times 16$ in. $\mathrm{NaI}(\mathrm{Tl})$ detector and GPS unit, was pulled by a person walking up and down Main St. See the bottom portion of Figure 1.3.1 for GPS data and detector count rates for one pass. Total count rates from these measurements are more consistent with the static cart measurements, but the GPS data is of very poor quality. There are gaps in reported position at semiregular intervals, making it very difficult to determine where the cart was as a function of time or to determine cart speed. The source of this consistent problem, which is present across all walking datasets, in GPS location reporting is not known. Because the detector location and speed are not known for these datasets, they are not useful to validate synthetic generation methodologies or particle transport detector response calculations.

The effect of the count rate reduction in the dynamic van measurements, most likely caused by the van itself, and error in GPS position reporting motivated the team to plan new dynamic measurements using a configuration as close to as possible to the static measurements and to use a system that can move at a constant speed. In MUSE 1, a robot pulled a detector cart, set to a constant speed, to create dynamic datasets that were used to validate synthetic data generation.

\subsection{GEOREFERENCING}

A differential GPS (DGPS) unit was used to obtain centimeter-accurate location measurements for the various measurement types, including static NaI(Tl), LIDAR, HPGe, and weather stations. This allowed for all measurement points to be georeferenced with a known latitude and longitude for repeatability of future measurements. For the static measurements, static points were measured along the $x$ and $y$ axes (Main St. and Church St.) as shown in Figure 1.4.1.

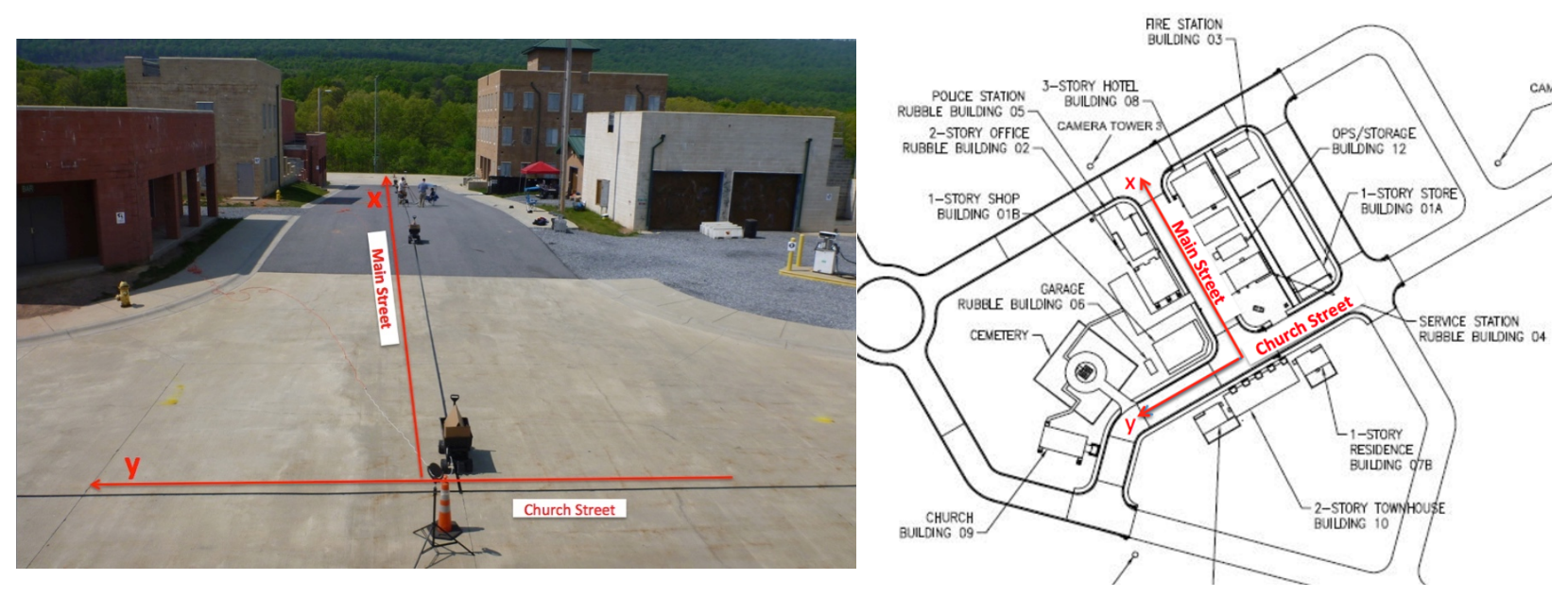

Figure 1.4.1. Cartesian layout of the CACTF.

The $x$ axis was $89 \mathrm{~m}$ long and GPS measurements were made in $1 \mathrm{~m}$ increments. All but one of the collections were processed within $1 \mathrm{~cm}$ accuracy, specifically $x=34 \mathrm{~m}$ was not within the $1 \mathrm{~cm}$ accuracy goal. The $y$ axis was $77 \mathrm{~m}$ long and was split into two parts; at the intersection of the $x$ axis, continuing $42 \mathrm{~m}$ in the positive direction and $35 \mathrm{~m}$ in the negative direction. The GPS measurements along the $y$ axis were also performed in $1 \mathrm{~m}$ increments, with the collections processed within $1 \mathrm{~cm}$ accuracy. A total of 50 LIDAR static points and numerous LIDAR target points were also processed to within $1 \mathrm{~cm}$. 
Approximately $39 \mathrm{HPGe}$ collection points were collected, but eight of these measurements failed to fall within $1 \mathrm{~cm}$ accuracy. The GPS locations of both meteorology stations, one each at the ROC and at Range 30 , as well as the radiological source point were measured to within $1 \mathrm{~cm}$ accuracy. All the DGPS measurement points characterized are shown in Figure 1.4.2.
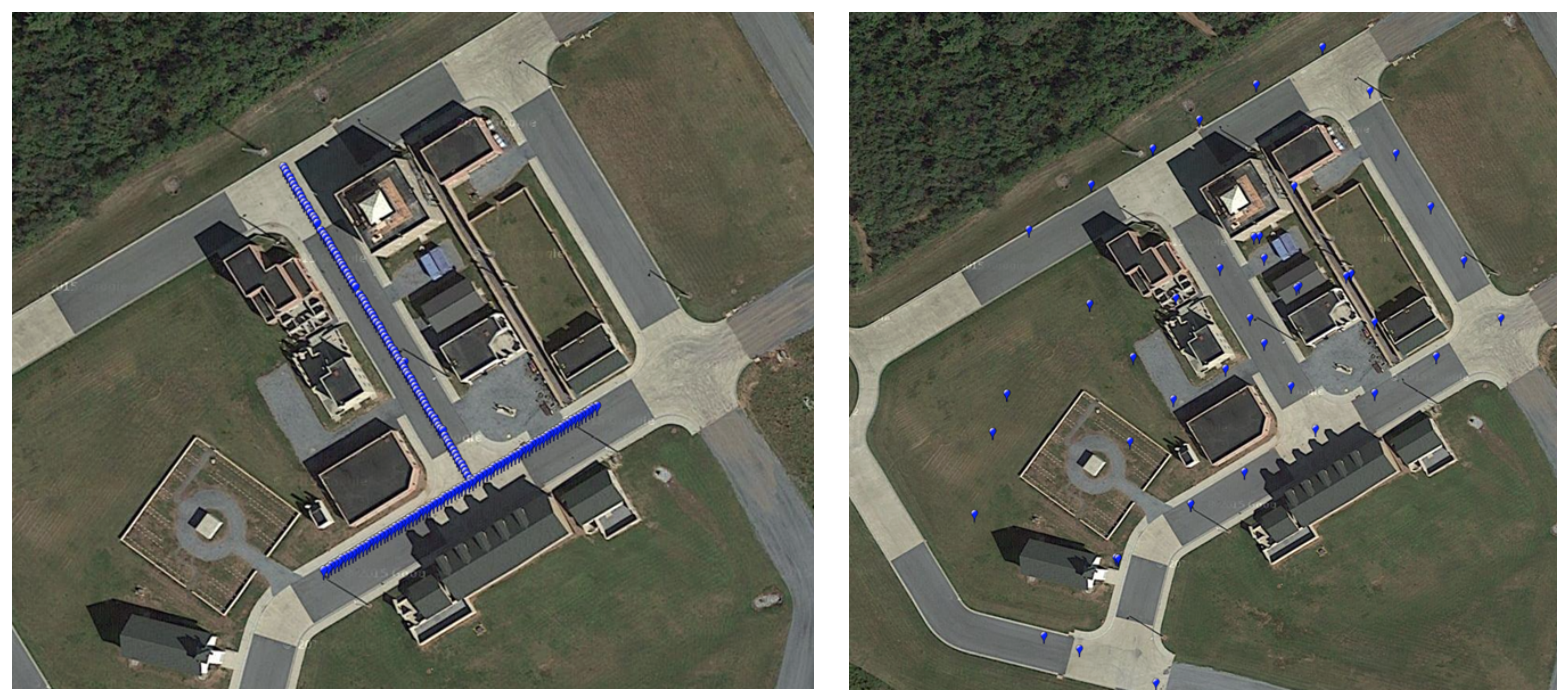

Figure 1.4.2. Differential GPS points for all NaI(TI), LIDAR, HPGe, and meteorology collection locations.

Along with the static measurements obtained, dynamic measurements were also performed. Analysis of the dynamic DGPS showed the accuracy to not have centimeter precision. This made the correlation of location along the roadway and the radiation data unreliable, and therefore it was not usable for model verification.

\subsection{LIDAR MAPPING OF CACTF BUILDING EXTERIORS}

An accurate representation of the physical environment is needed to create the realistic models for representing the effects of that environment on radiation measurements. Although architectural drawings and physical site measurements can provide accurate results, the conversion of these to a realistic model can be time-consuming and leave room for error. An alternative to this approach is to use a laser scanner to capture three-dimensional (3-D) data to generate such models, which also allows all other objects in the environment not accounted for in drawings to be accurately captured and represented virtually. For the measurements at FTIG, LIDAR data was taken with the objective of capturing the geometry of the exterior buildings and nearby terrain.

A man-portable long-dwell Zoller+Frölich (Z+F) model 5006 3-D Laser Scanner (Figure 1.5.1) was used to collect the LIDAR data. This unit has a rotating mirror for vertical scanning and a rotating head for horizontal scanning to allow the 5006 system to capture up to $310^{\circ} \times 360^{\circ}$ field-of-view data that consists of surface reflectance and range. For the recreation of a model, the range data obtained from the LIDAR is a key element collected. A point cloud is generated from the LIDAR collections and consists of the discrete $x, y$ and $z$ coordinates of the surfaces that the laser scans at the angular resolutions used for the scans. The closer the objects or surfaces are to the scanner, the denser the point cloud will be. With the use of targets or common points present in scans from multiple positions, multiple scans at different positions can be registered or aligned with each other to create larger point clouds. The target placement and registration process can also be used to scan objects from different viewing perspectives. 
A)

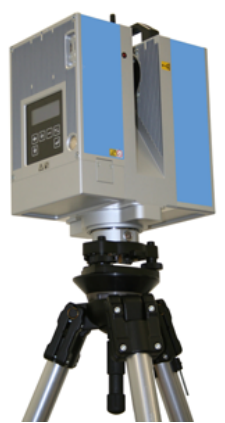

B)

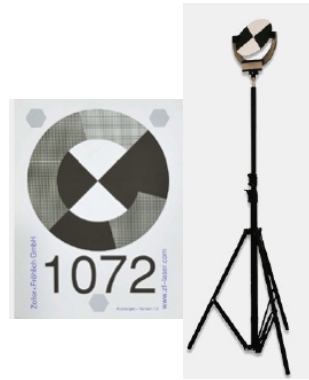

Figure 1.5.1. DGPS unit employed during measurement campaign. (A) $\mathrm{Z}+\mathrm{F}$ model 5006 LIDAR. (B) Example of stationary and nonstationary targets used in LIDAR collection.

A total of 52 scans were conducted at strategic locations around the town center using the long-dwell high-fidelity Z+F Imager system. Multiple optical images were obtained at each LIDAR scan position along with other various locations around the site, as shown in Figure 1.5.2.
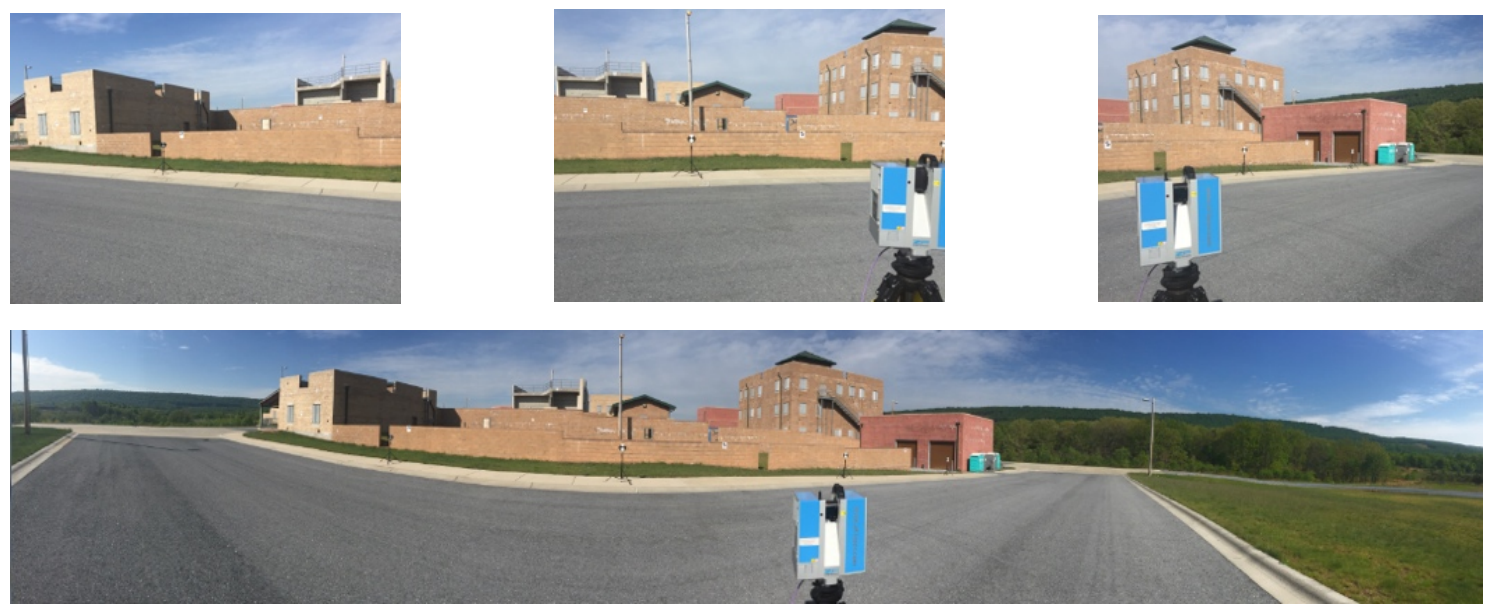

Figure 1.5.2. Optical images showing different perspectives of a LIDAR scan position.

The individual scans were registered (i.e., stitched together) using target overlap into a single comprehensive point cloud containing 82 million points as shown in Figure 1.5.3. 


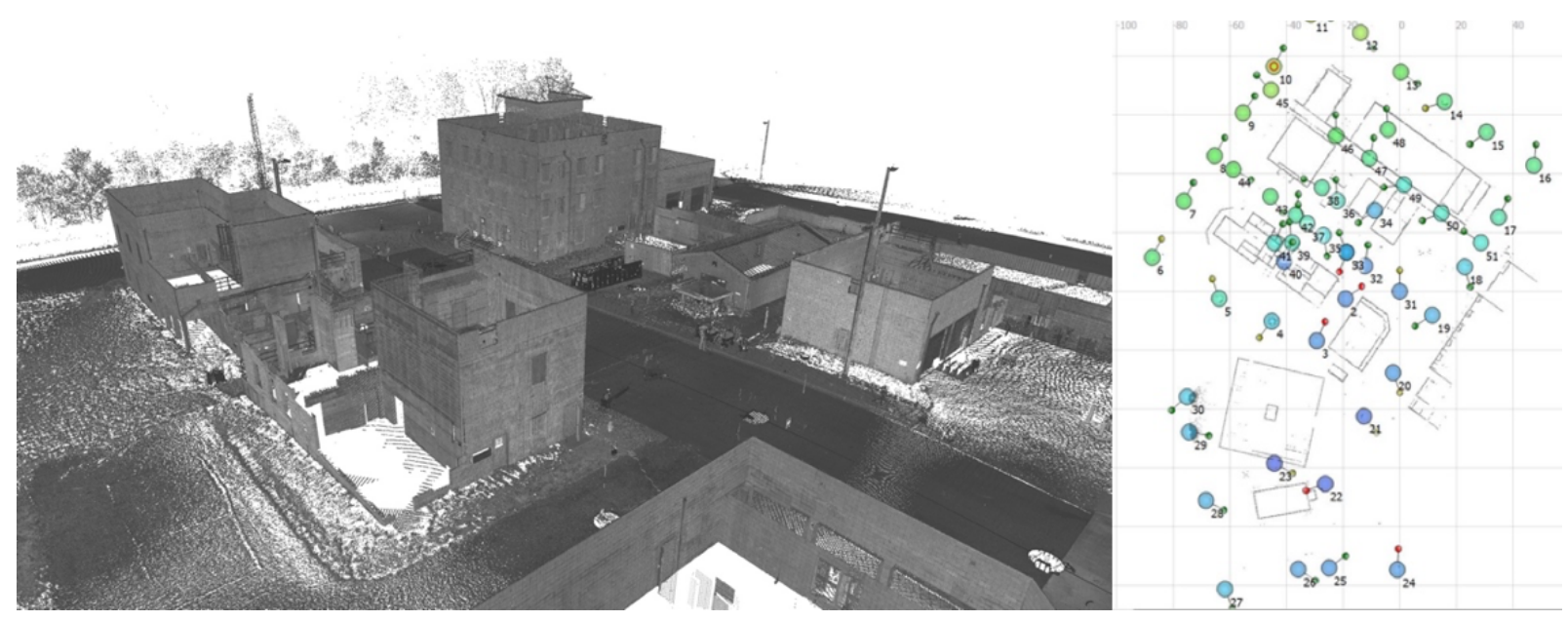

Figure 1.5.3. LIDAR image and measurement locations created of CACTF. (Left) Composite point cloud of FTIG with high-resolution LIDAR. (Right) Locations where individual scans were taken are marked.

This point cloud has served as ground truth for evaluating data from subsequent campaigns. In addition, this point cloud was used as source data for the derivation of a manually extracted CAD model as shown in Figure 1.5.4. The CAD model was subsequently meshed for ingestion into the GEANT4 ${ }^{1}$ radiation transport code. Isolated imagery was used to fill in a few gaps in the LIDAR, and architectural plans were used to estimate unseen elements such as wall thickness of structures.

${ }^{1}$ GEANT4, GEOmetry ANd Tracking, is a toolkit developed and maintained by CERN for the simulating particle transport through matter via Monte Carlo methods. 

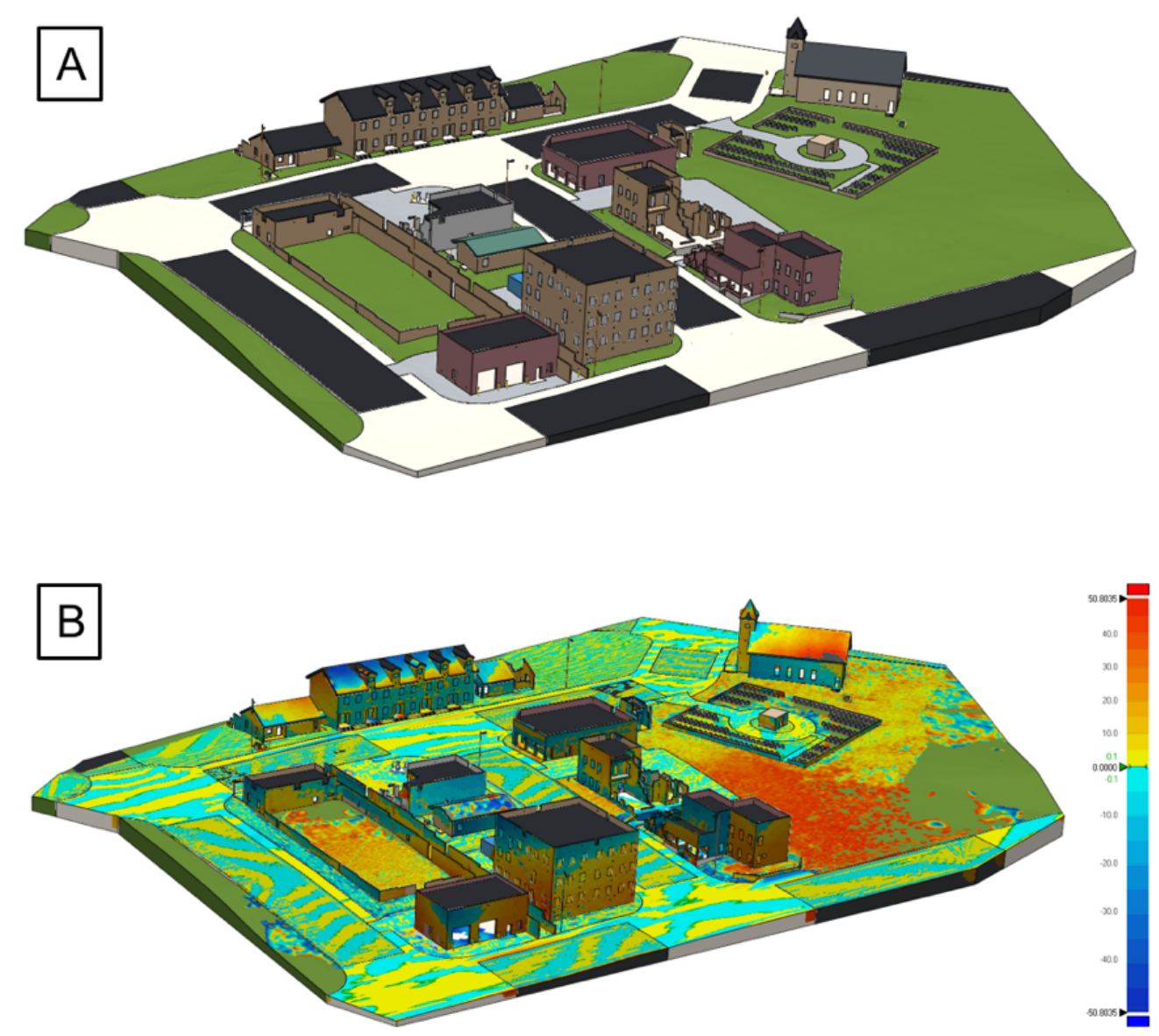

Figure 1.5.4. CAD model created of CACTF using OPTUS 1 imaging measurements. $(A)$ CAD model manually derived from ground truth point cloud. $(B)$ Spatially resolved comparison of the mismatch between the point cloud and the CAD model.

\subsection{METEOROLOGICAL AND PERSISTENT RADIATION MONITORING}

Correlated meteorology and persistent radiation data were obtained at two locations at FTIG shown in Figure 1.6.1. The primary objectives of these measurements were (1) to complement the measurement of gamma-ray backgrounds at the CACTF by monitoring area radiation with a persistent detector system and using it as a reference for anomalies, (2) to study potential weather and environment-related effects on measurements of natural gamma-ray background spectra and establish systematic uncertainties caused by them, (3) to study the variation in weather observables and correlated radiation effects over spatially separated systems, and (4) to understand how close a weather station needs to be to provide relevant information. To study these effects, two separate RSI-700 systems, each coupled with an Oak Ridge National Laboratory (ORNL) Davis Pro 2 weather system, were used. The two Davis weather systems were benchmarked at the site with co-located meteorological grade weather stations. 


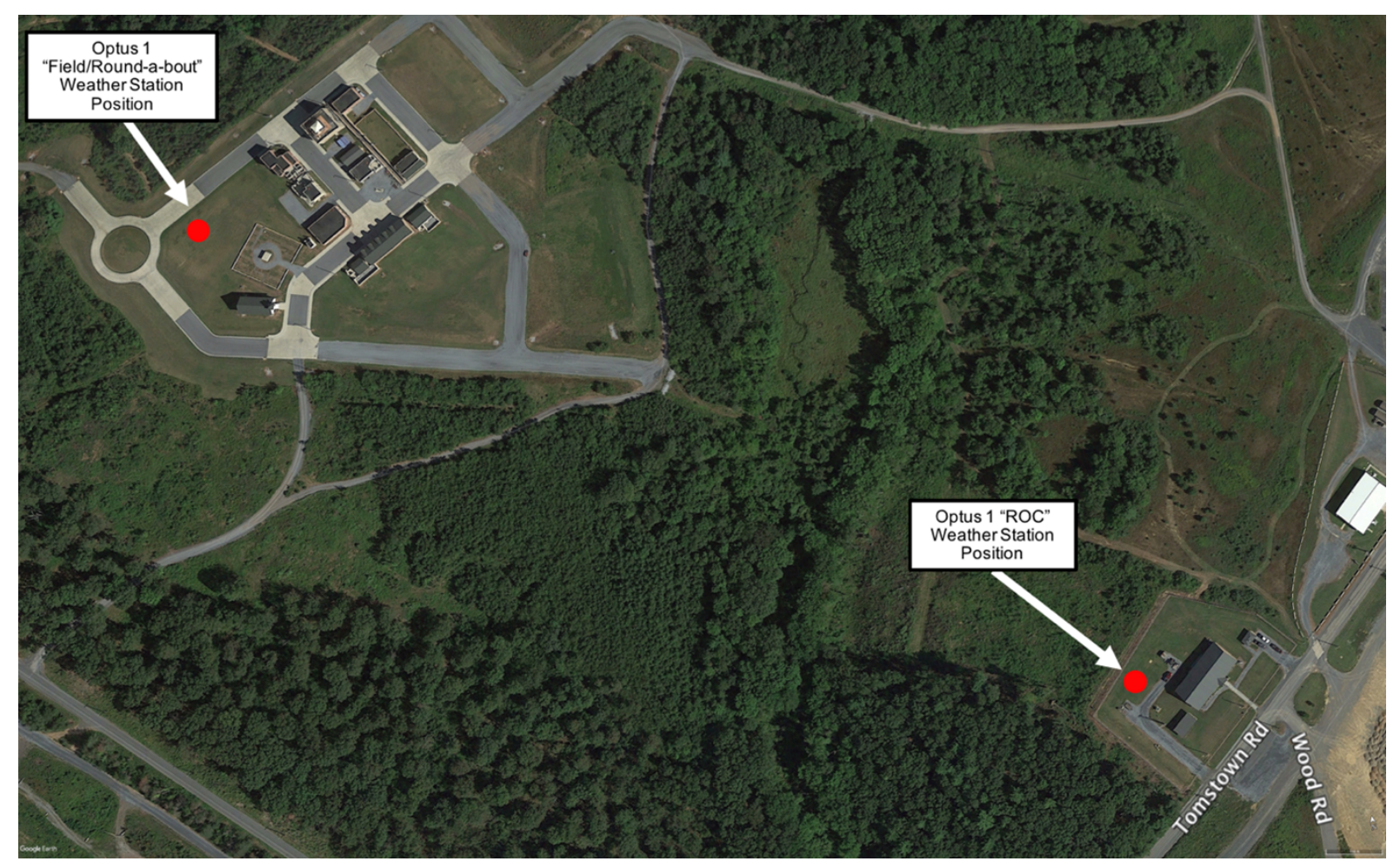

Figure 1.6.1. Weather station locations for OPTUS 1 campaign.

Data collected by the RSI $\mathrm{NaI}(\mathrm{Tl})$ detector system that was placed at the roundabout next to Davis 2 weather system came in terms of 10 large $(\sim 250 \mathrm{MB})$.csv files. Each line inside the .csv file corresponds to approximately $1 \mathrm{~s}$ of recorded data and provides raw counts per second distributed between 1,023 bins from $(0,1023)$, equivalent to 10-bit ADC. There is significant time coverage overlap between consecutively recorded .csv files; therefore, significant bookkeeping and care was taken when reading data to avoid double counting. The recorded spectra were added second-by-second in $5 \mathrm{~min}$. intervals and then correlated to the corresponding weather file readings, which reports weather parameters every 5 min. Using the recorded times from each unit, the data was correlated and combined in ROOT TTree ${ }^{2}$ structure format for further analysis. Various weather parameters were analyzed to determine possible correlation with radiation data. For example, Figure 1.6.2 shows total spectra and ${ }^{214} \mathrm{Bi},{ }^{40} \mathrm{~K}$, and ${ }^{232} \mathrm{Th}$ region of interest (ROI) counts versus time overlaid with air temperature (top) and rain rate readings (bottom).

\footnotetext{
${ }^{2}$ ROOT is a data analysis software suite developed and maintained by CERN for particle physics applications. TTree class within ROOT provides the user with an optimized method to search, analyze, and store large datasets while reducing required disk space and enhancing access speed.
} 

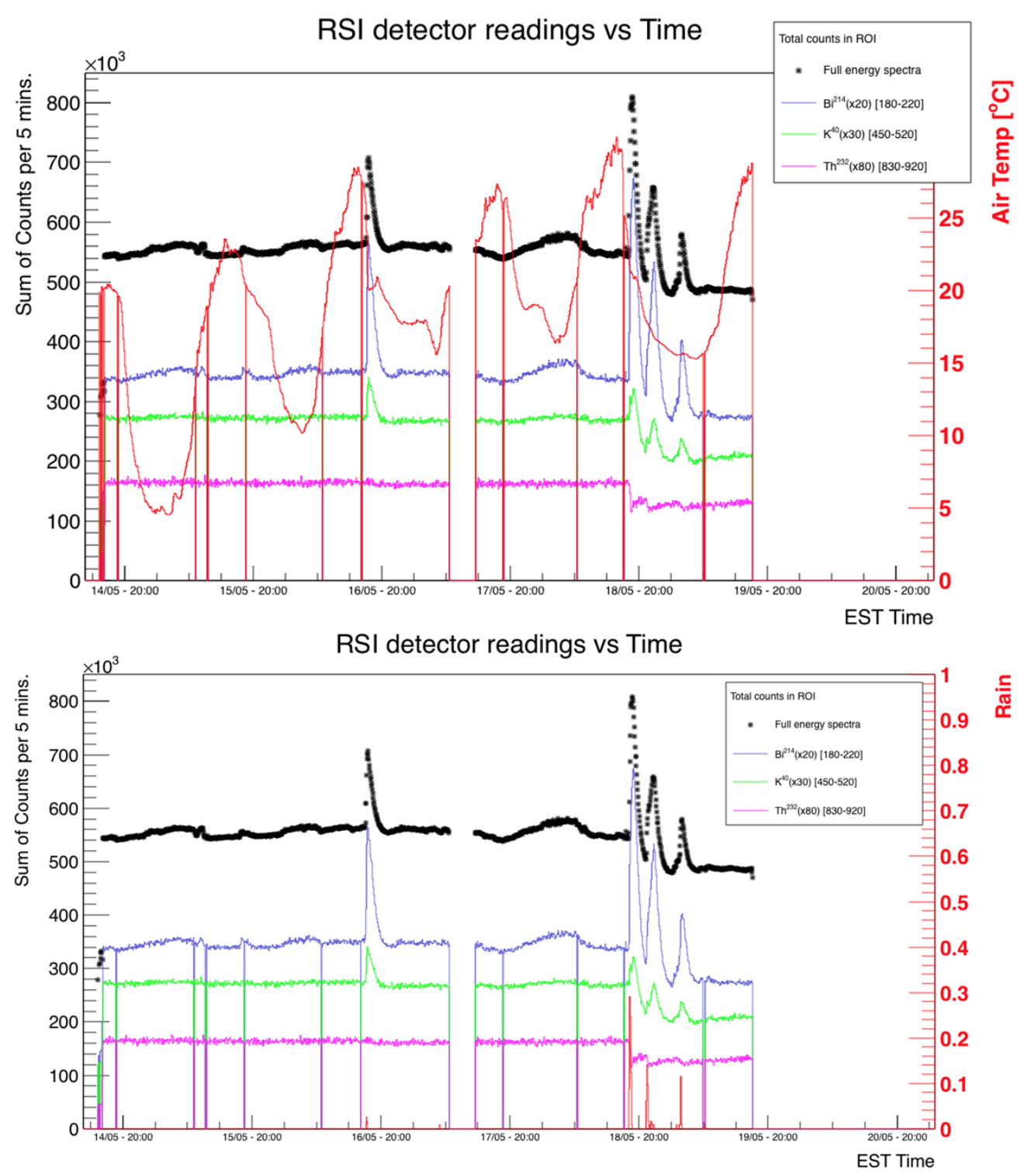

Figure 1.6.2. RSI Detector count rate versus air temperature (above) and count rate versus rainfall (below).

From the rain rate comparison in Figure 1.6.2, several spikes in the count rates can be observed during and after rainfall. There are other fluctuations in the count rate data that are caused by either natural (environmental) reasons or by the ${ }^{137} \mathrm{Cs}$ source being moved around the FTIG area. Although the rain and count rate show an obvious relationship, other environmental parameters might contribute to the count rate fluctuation, but more long-term data need to be collected to determine these correlations.

Figure 1.6.2 shows several rain events occurring on two separate days, May 16 and May 18, respectively. The rain event on May 16 was a small rain $(<0.05$ in.), but it caused the total count rate observed in the detector to rise by approximately $35 \%$ during the event. Approximately $4-5 \mathrm{~h}$ after the rain event, the count rate returned to the same as before the rain. 

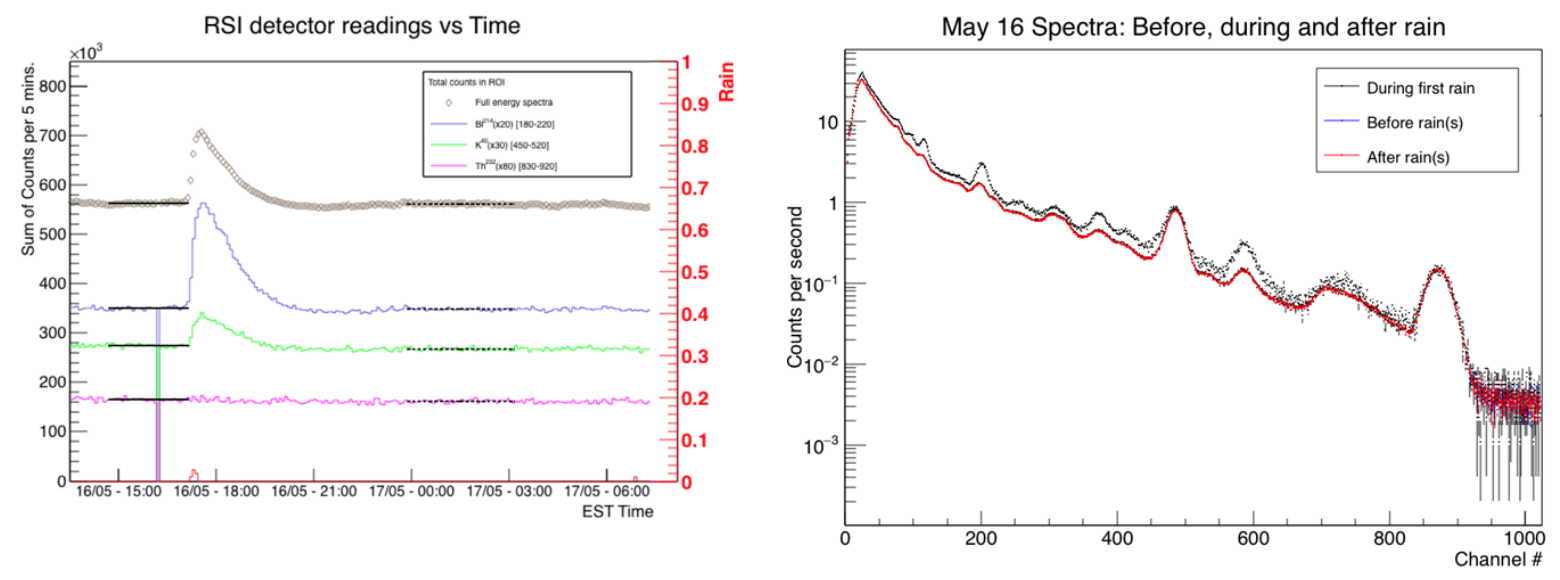

Figure 1.6.3. Spectrums collected during May 16 rain event. (Left) Counts per 5 min. versus time zoomed. (Right) Raw gamma-ray spectra before, during, and after the rain.

On May 18th, there were multiple rain events that occurred consecutively over a $12 \mathrm{~h}$ span of time. As on May 16th, an elevated count rate spike was observed during these rain events. This rain event accrued nearly $0.6 \mathrm{in}$. of rain over the $12 \mathrm{~h}$ period compared to the $<0.05 \mathrm{in}$. on the previous day. The count rate after the rain measured to be $>10 \%$ below the count rate before the rain (Figure 1.6.4). This decrease is assumed to be from the saturation of the soil below the radiation sensor to be large enough to attenuate the background counts from the soil. Although this assumption seems like a reasonable explanation, it cannot be verified without soil moisture sensors to monitor the saturation of the ground. In OPTUS 2 and MUSE 1, a soil moisture sensor was included with the weather system.
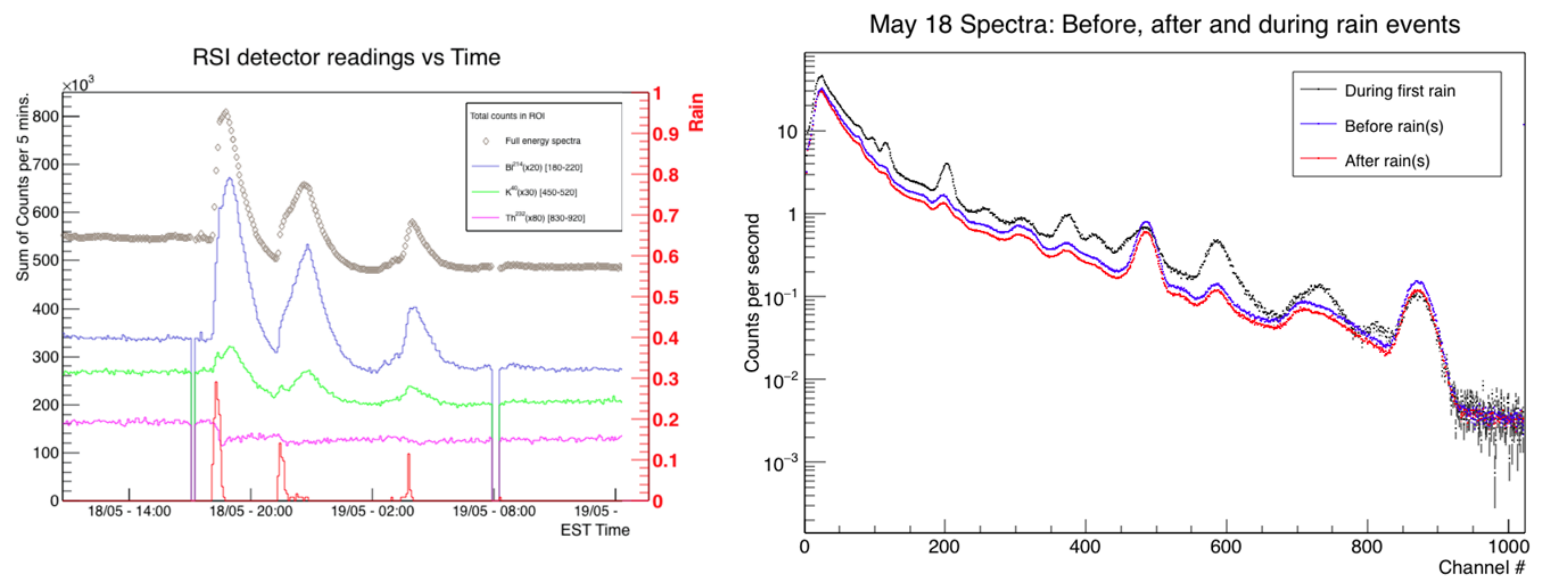

Figure 1.6.4. Spectrums collected during May 18 rain event. (Left) Counts per 5 min. versus time zoomed. (Right) Raw gamma-ray spectra before, during and after the rain. 


\section{OPTUS 2 MEASUREMENT CAMPAIGN}

The second data collection, OPTUS 2, was performed at FTIG in August 2015 and targeted to collect further refined data including those determined needed from the analysis of OPTUS 1 data. Additional shielded HPGe measurements were performed to evaluate the variation of NORM values for similar materials across the site. Some duplicate measurements were performed with the 2 in. $\times 4$ in. $\times 16$ in. $\mathrm{NaI}(\mathrm{Tl})$ detectors to correlate with static data from OPTUS 1. Further NaI(Tl) measurements also included those performed in the interior of the hotel building with and without a source for modeling validation of the hotel. LIDAR data was taken in the interior of the hotel as well to accompany the interior $\mathrm{NaI}(\mathrm{Tl})$ data for model reconstruction. From OPTUS 1, it was determined the weather patterns varied from the ROC to the CACTF. Therefore, the persistent radiation and meteorological monitoring stations were both placed on the CACTF range to monitor the radiation response due to weather phenomena that occurred near the measurements being performed. A shielded directional $\mathrm{NaI}(\mathrm{Tl})$ detector was used to determine the relative directional background source term seen by the solid angle of the detector. Neutron measurements were also taken to evaluate the importance of background neutron collection at the site for future radiation transport modeling.

\subsection{HPGe OUTDOOR BUILDING AND GROUND SURFACE MEASUREMENTS}

During OPTUS 2, 36 additional shielded HPGe measurements were taken at the FTIG MOUT facility in a similar manner to those conducted during OPTUS 1. One of the important differences was the implementation of an air blower and small wooden planks to facilitate the cooling of the detector throughout the numerous measurements taken each day at the FTIG site. A picture of one of these measurements can be seen in Figure 4.1.2, showing the lead sarcophagus elevated on the wooden planks and the air hose inserted at the top of the lead cave. In addition, a tent was added to reduce solar heating. A comprehensive list of the HPGe measurements taken at OPTUS 2 is shown in Table 2.1.1. The measurements denoted with an asterisk represent a measurement repeated from OPTUS 1, whereas the two measurements denoted with double asterisks represent the only two measurements not used in the final NORM determination analysis. H54 was taken with a different detector than all the other measurements, and so was left out of the analysis, whereas $\mathrm{H} 64$ was a measurement when a ${ }^{137} \mathrm{Cs}$ source was nearby. This leaves 34 measurements to add to the 19 from the OPTUS 1 campaign — giving 53 measurements in total.

Table 2.1.1. Comprehensive list of OPTUS 2 HPGe measurements.

\begin{tabular}{|c|c|c|c|}
\hline ID & Material & Description & File Name \\
\hline H09* & Concrete, sidewalk & 1-story shop (bar) on Church St. side & "H9_150811_1218 \\
\hline $\mathrm{H} 10^{*}$ & Asphalt & Main St., in front of 2-story office & H10_150811_1526 \\
\hline H11* & Block, light tan & $\begin{array}{l}2 \text {-story office, side facing } 1 \text {-story shop, } * \text { detector resting } \\
\text { on ground }\end{array}$ & H11_150811_1346 \\
\hline $\mathrm{H} 12 *$ & Gravel & Service station, $0.75-1$ in. thick & H12_150811_1659 \\
\hline H17* & Gravel & Between 2-story office and 1-story shop, 3 in. thick & H17_150811_1439 \\
\hline H18* & Soil & 2-story office, side facing 1-story shop & H18_150811_1615 \\
\hline $\mathrm{H} 20^{*}$ & Block, dark tan & OPS/storage building, service station side & H20_150813_0843 \\
\hline $\mathrm{H} 21^{*}$ & Concrete, sidewalk & Between OPS/storage and dumpster area & H21_150813_1006 \\
\hline $\mathrm{H} 23^{*}$ & Gravel & Dumpster area, where sidewalk should be & H23_150813_1051 \\
\hline $\mathrm{H} 24 *$ & Concrete, sidewalk & Police station, in front of main stairs & H24_150812_0848 \\
\hline
\end{tabular}


Table 2.1.1. Comprehensive list of OPTUS 2 HPGe measurements (continued).

\begin{tabular}{|c|c|c|c|}
\hline ID & Material & Description & File Name \\
\hline $\mathrm{H} 25^{*}$ & Concrete, sidewalk & 3-story hotel, in front of main stairs & H25_150812_1613 \\
\hline $\mathrm{H} 28^{*}$ & Block, grey & 3-story hotel, wall on front porch, facing inwards & H28_150813_1225 \\
\hline $\mathrm{H} 29^{*}$ & $\begin{array}{l}\text { Concrete, } \\
\text { intersection }\end{array}$ & Intersection of Main and Maple, near police station & H29_150812_0931 \\
\hline $\mathrm{H} 40$ & Asphalt & Main St., in front of OPS/storage building 12 & H40_150813_0924 \\
\hline $\mathrm{H} 42$ & Concrete, patio & Patio in front of $\mathrm{TH} 3$ & H42_150813_1433 \\
\hline $\mathrm{H} 43$ & Block, dark tan & $\begin{array}{l}2 \text {-story townhouse building } 10 \text {, outer north wall, facing } \\
\text { inwards }\end{array}$ & H43_150813_1515 \\
\hline $\mathrm{H} 44$ & Concrete, sidewalk & 2-story townhouse, in front of $\mathrm{TH} 3$ & H44_150813_1352 \\
\hline $\mathrm{H} 45$ & Asphalt & Maple St., behind police station & H45_150812_1013 \\
\hline $\mathrm{H} 46$ & Concrete, sidewalk & North of police station, on Maple St. side & H46_150812_1330 \\
\hline H47 & Soil & Soil behind/north of police station next to Maple St. & H47_150812_1408 \\
\hline $\mathrm{H} 49$ & Asphalt & Maple St., in front of hotel & H49_150812_1057 \\
\hline $\mathrm{H} 50$ & Concrete, sidewalk & North of hotel on concrete sidewalk on Maple St. & H50_150812_1139 \\
\hline H51 & Soil & Soil between hotel and sidewalk, North side (Maple St.) & H51_150812_1236 \\
\hline $\mathrm{H} 54^{* *}$ & $\begin{array}{l}\text { Concrete, } \\
\text { foundation }\end{array}$ & North of hotel, grey concrete wall (Cathy's machine) & H54_150812_1524 \\
\hline H58 & Soil & Soil between townhouse and sidewalk & H58_150813_1558 \\
\hline H59 & Soil & Soil between hotel and sidewalk, West side (Main St.) & H59_150813_1135 \\
\hline H60 & Concrete, floor & 3 -story hotel, room 105 , floor over basement & H60_150814_0937 \\
\hline H61 & $\begin{array}{l}\text { Concrete, } \\
\text { foundation }\end{array}$ & 3 -story hotel, room 105 , floor over foundation & H61_150814_1021 \\
\hline H62 & Block, grey & 3-story hotel, room 105, wall facing room 106 & H62_150814_1112 \\
\hline H63 & Concrete, floor & 3-story hotel, room 109, floor over basement & H63_140815_1205 \\
\hline $\mathrm{H} 64 * *$ & Concrete, floor & $\begin{array}{l}\text { 3-story hotel, room } 109 \text {, floor over basement, } \\
\text { with } 40 \mu \mathrm{Ci} \text { source in basement }\end{array}$ & H64_140815_1333 \\
\hline H65 & Asphalt & $\begin{array}{l}\text { Road B (between bar and residence with rubble); } \\
x=0 \mathrm{~m}, y=14 \mathrm{~m}\end{array}$ & H65_150815_0855 \\
\hline H66 & Asphalt & $\begin{array}{l}\text { Road B (between bar and residence with rubble); } \\
x=0 \mathrm{~m}, y=16 \mathrm{~m}\end{array}$ & H66_150815_0940 \\
\hline H67 & Asphalt & $\begin{array}{l}\text { Road B (between bar and residence with rubble); } \\
x=0 \mathrm{~m}, y=18 \mathrm{~m}\end{array}$ & H67_150815_1019 \\
\hline H68 & Asphalt & $\begin{array}{l}\text { Road B (between bar and residence with rubble); } \\
x=0 \mathrm{~m}, y=20 \mathrm{~m}\end{array}$ & H68_150815_1101 \\
\hline H69 & Asphalt & $\begin{array}{l}\text { Road B (between bar and residence with rubble); } \\
x=0 \mathrm{~m}, y=22 \mathrm{~m}\end{array}$ & H69_150815_1145 \\
\hline
\end{tabular}

NORM count rates for ${ }^{214} \mathrm{Bi},{ }^{40} \mathrm{~K}$, and ${ }^{232} \mathrm{Th}\left({ }^{208} \mathrm{Tl}\right)$ extracted from the HPGe measurements and those taken at OPTUS 1 are shown in Figure 2.1.1. These measurements add upon those taken in OPTUS 1, filling in many of the gaps left from this first campaign. Upon analysis of the measurements conducted at OPTUS 2, two remaining major deficiencies were identified. The first deficiency is the distinct lack of measurements of the concrete intersections, which seemed of prime importance since the NaI(Tl) data 
shows background variability in these areas. Even after OPTUS 2, there were only three measurements of the concrete intersections, one of which (H36) appeared to be an outlier. The other deficiency was with respect to the cinderblock walls, where the scientists making the measurements were not necessarily aware of the methodology that would ultimately be used to estimate the NORM concentrations in these walls. In short, measurements of an outward facing wall made the NORM determination easier and likely more accurate because less assumptions had to be made about the contribution of material on the other side of the wall contributing to the measured spectra. This brought about the desire for additional wall measurements where the detector was on the inside of the wall, facing the exterior of the building. There was also a minor concern that the few measurements of the gravel lots were of varying gravel depths, so more consistent measurements were desired of the gravel as well. More discussion on this methodology and the justification for these additional wall measurements can also be found in Section 4.1.
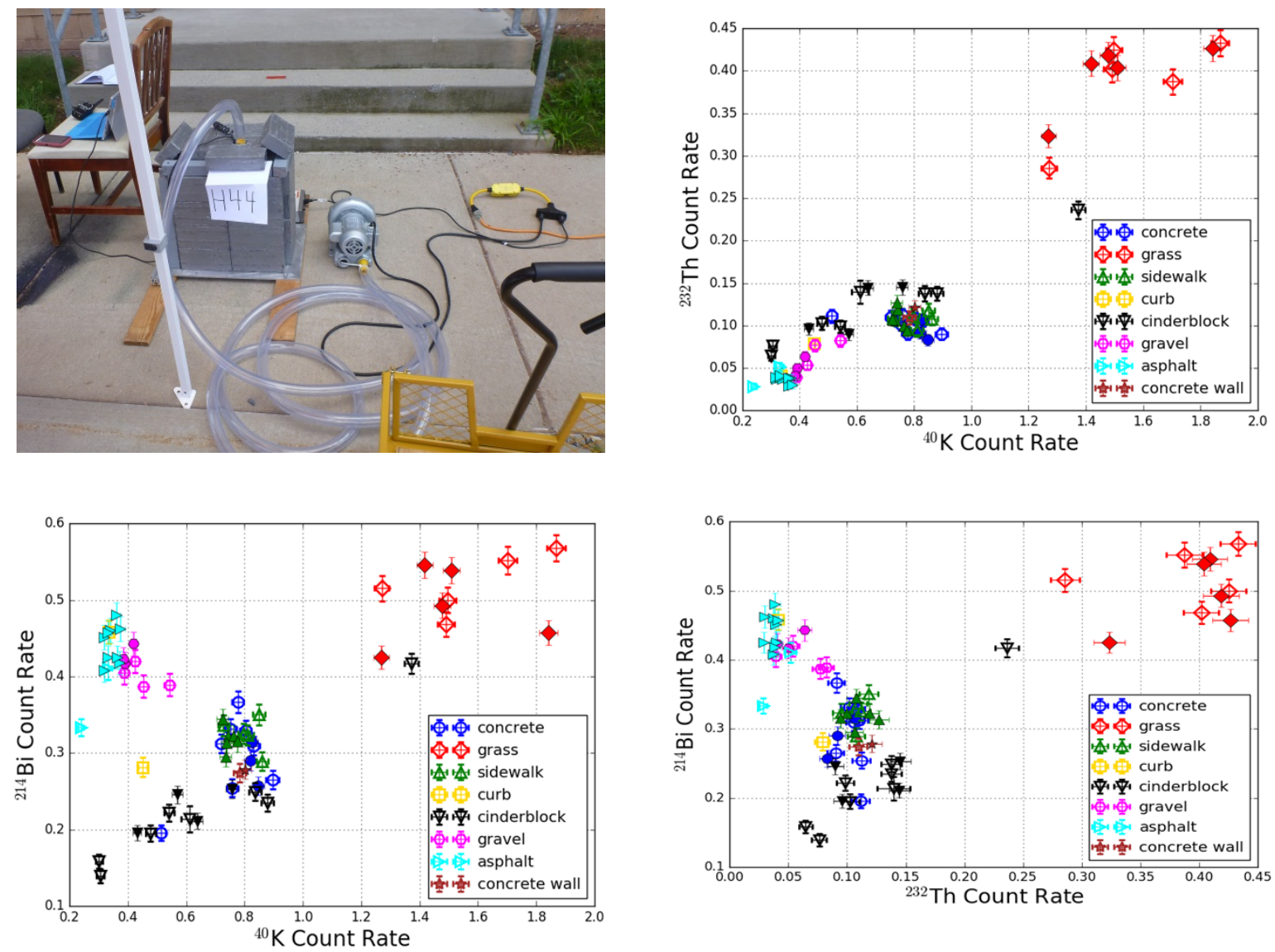

Figure 2.1.1. (Top left) A picture of one of the HPGe measurements conducted as part of the OPTUS 2. (Top right) Correlation between $1,460 \mathrm{keV}{ }^{40} \mathrm{~K}$ and $2,614 \mathrm{keV}{ }^{232} \mathrm{Th}\left({ }^{208} \mathrm{Tl}\right)$ peak areas for the OPTUS 1

measurement campaign. (Bottom left) Correlation between $1,460 \mathrm{keV}{ }^{40} \mathrm{~K}$ and $609 \mathrm{keV}{ }^{214} \mathrm{Bi}$ peak areas. (Bottom right) Correlation between $2,614 \mathrm{keV}{ }^{232} \mathrm{Th}\left({ }^{208} \mathrm{Tl}\right)$ and $609 \mathrm{keV}{ }^{214} \mathrm{Bi}$ peak areas. For all figures, open markers denote OPTUS 1 measurements and closed denote OPTUS 2. 


\subsection{NaI(TI) STATIC MEASUREMENTS}

After OPTUS 1, gain stabilization was included in the RUNE ${ }^{3}$ data acquisition system. Unfortunately, a different error, discovered after the OPTUS 2 measurement campaign, was introduced in the data acquisition system, which deleted some portions of $\mathrm{NaI}(\mathrm{Tl})$ list mode data stream. The amount of information lost was not constant over time and depended on the detector count rate. This resulted in a decreased count rate compared with OPTUS 1. See Figure 2.2.1 for the total count rates as a function of measurement location. Because of this error, this data is not suitable for further analysis. To avoid similar issues in the future, a benchmark set of laboratory measurements was created. Before each measurement campaign, the RUNE data acquisition system will be tested against these benchmarks to verify system integrity.

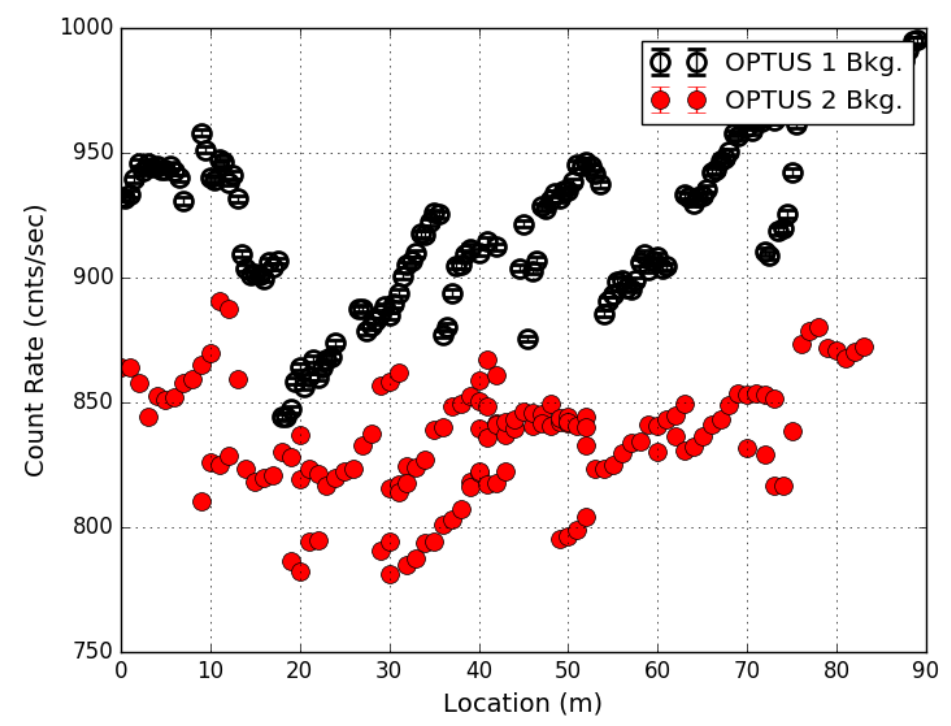

Figure 2.2.1. Static background count rate comparison for OPTUS 1 (empty black circles) and OPTUS 2 (filled red) for the same positions down Main St.

\subsection{NaI(TI) INDOOR MEASUREMENTS}

Measurements were performed throughout the interior of the hotel using the 2 in. $\times 4$ in. $\times 16$ in. $\mathrm{NaI}(\mathrm{Tl})$ detector systems. A total of 125 static measurements were performed inside the hotel. These measurements included background and source $\left(\sim 40 \mathrm{mCi}{ }^{137} \mathrm{Cs}\right)$ measurements spanning all the floors and roof of the building. The goal of these measurements was to determine the detector response with attenuation through the building material (floors and walls) to be compared to a modeled detector response inside the hotel. The various detector and source positions for each floor are shown in Figure 2.3.1.

\footnotetext{
${ }^{3}$ RUNE is a versatile toolkit developed and maintained by Oak Ridge National Laboratory. RUNE is used for radiation detection systems and wireless communications which can be employed for any vendor supplied system or any number of detection platforms simultaneously used within the field.
} 


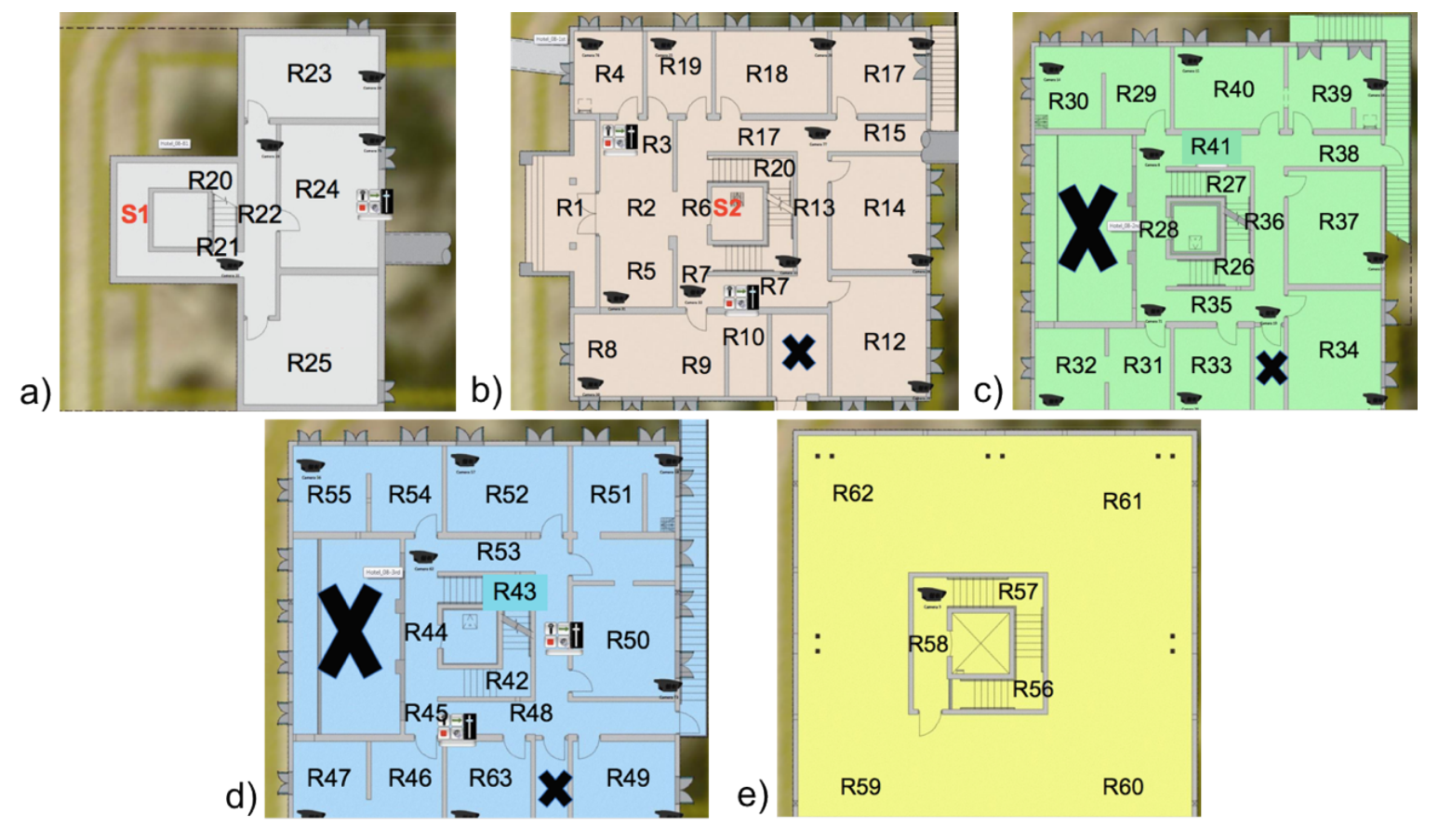

Figure 2.3.1. Detector (black) and source (red) locations for point throughout the hotel. (a) Basement, (b) first floor, (c) second floor, (d) third floor, and (e) roof. R\# denotes the sensor position, S\# denotes the source position.

As a result of the systematic error discussed in 2.2, the data obtained in the hotel cannot be used to verify the background in a model of the hotel; however, it still gives an approximation of the detector response because of a source at the various locations. Since the error is dependent on the detector count rate, the presence of a source influenced the results when the detector was near, thus measurements near the source likely have a larger error than those further away. Nonetheless, the data was analyzed to approximate the relative detector response one would see if a $\sim 40 \mathrm{mCi}$ of ${ }^{137} \mathrm{Cs}$ was placed at different locations in a model of the hotel interior. A comparison of the spectral response of the system shown in Figure 2.3.2 and Figure 2.3.3 for two of the source locations ( 1 1 and S2). Figure 2.3.2 illustrates the spectral comparison detectors placed in the stairwell locations on each floor with the source placed on the opposite side of the elevator wall to first stairwell landing in the basement (S1). Figure 2.3.3 provides the spectral comparison detectors placed near the elevator shaft on each floor with the source placed at the elevator shaft on the first floor (S2). 


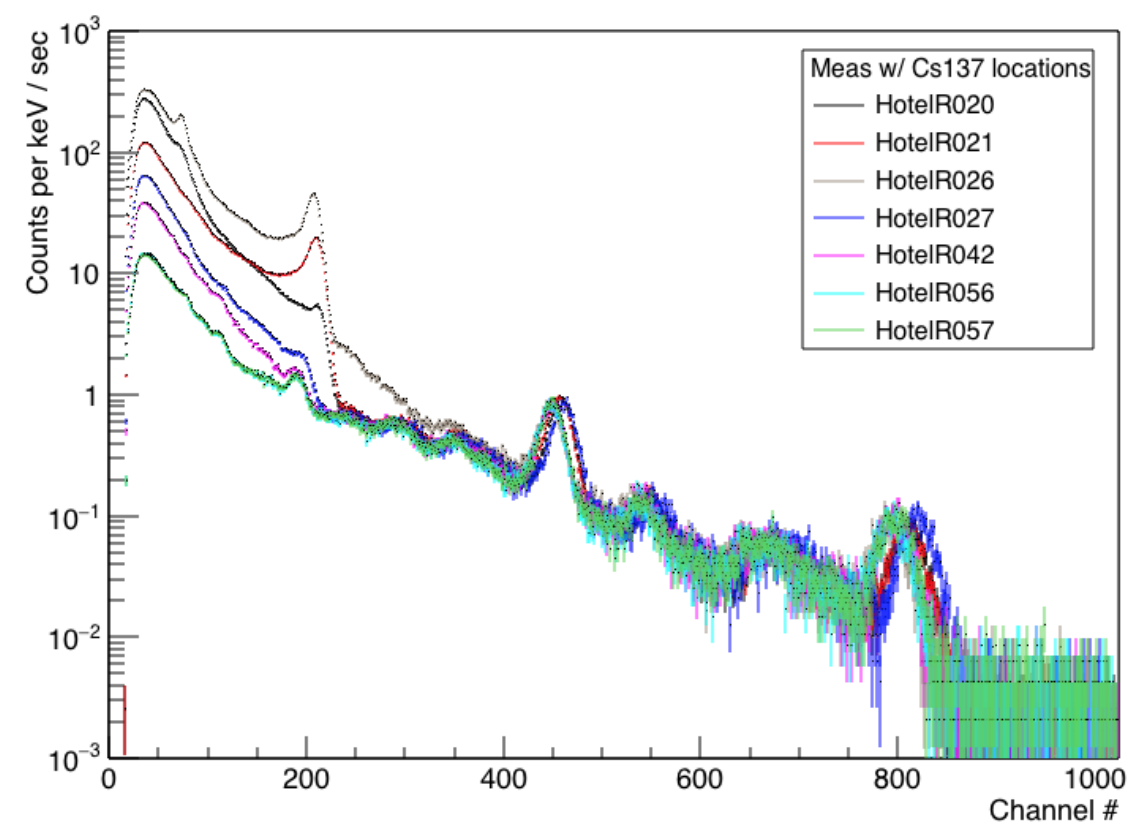

Figure 2.3.2. Stairwell detector response comparisons for source location S1.

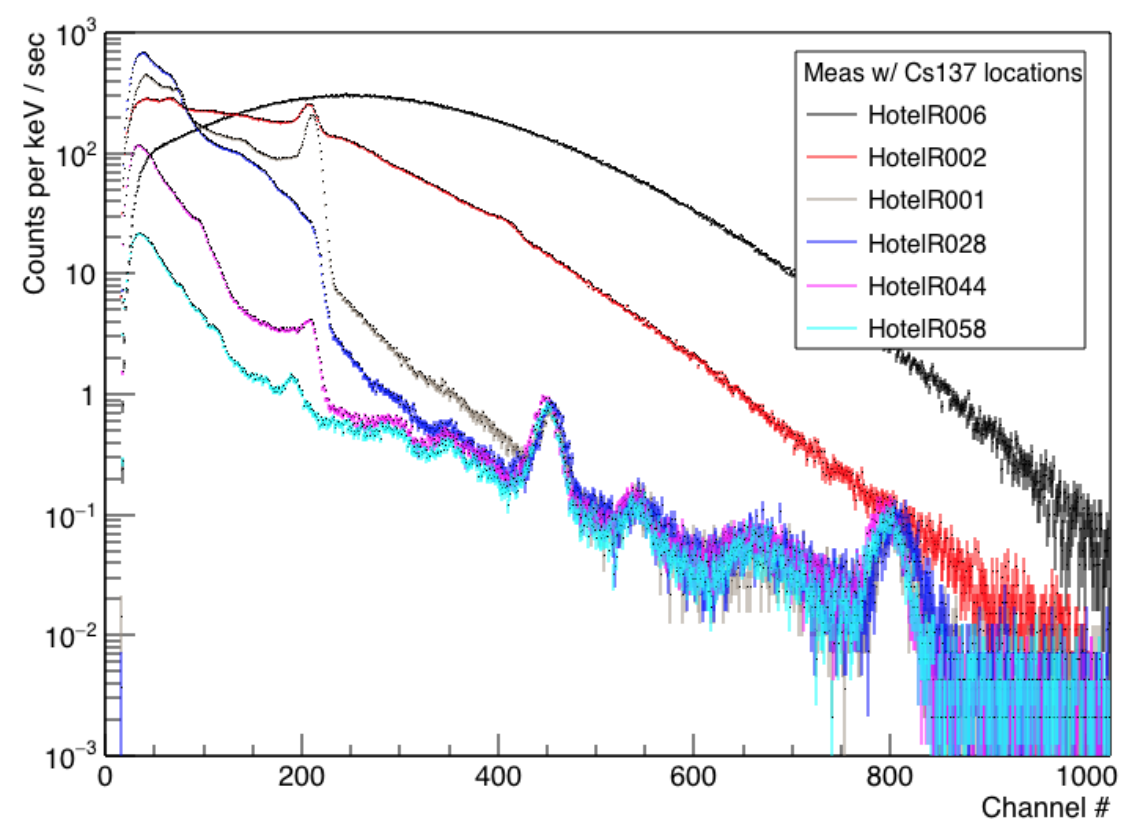

Figure 2.3.3. Near-elevator detector response comparisons for source location S2.

From Figure 2.3.2 it is evident that the when the source was placed at position $\mathrm{S} 1$ in the basement, the $662 \mathrm{keV}$ peak was prominent until the second floor (R26), and the Compton scattered events were apparent through parts of the third-floor stairwell (R42). The measurements at the stairwell near the roof level (R56 and R57) had enough building attenuation and distance from the source that their responses were equivalent to background.

The second source location (S2) was analyzed in Figure 2.3.3 to measure the attenuation of the source near the elevator shaft and shows the spectra of the detectors at locations near the elevator shaft openings 
on each floor. Placing the detector at the same location of the source (R6 and S2), it is evident that the source overwhelmed the detector, and all that is visible is pile up in the detector. The measurement on the second floor (R28) has some counts in the $662 \mathrm{keV}$ peak but is dominated by scattering from the source that resulted from the detector being placed directly above the source with reinforced concrete flooring between the two objects. The third-floor measurement (R44) has a more prominent peak at $662 \mathrm{keV}$ and less scattering in the continuum because of the additional floor between the source and detector. The fourth-floor measurement (R58) did not have any counts above background from the ${ }^{137} \mathrm{Cs}$ source.

\subsection{DIRECTIONAL NaI(TI) BACKGROUND SOURCE TERM MEASUREMENTS}

A directional detection setup was explored to evaluate an alternative approach to determine the source of background radiation. A 2 in. $\times 4$ in. $\times 16$ in. $\mathrm{NaI}(\mathrm{Tl})$ detector was surrounded with 2 in. of lead on all sides, and measurements were performed with one face of the detector open at a time as shown in Figure 2.4.1.

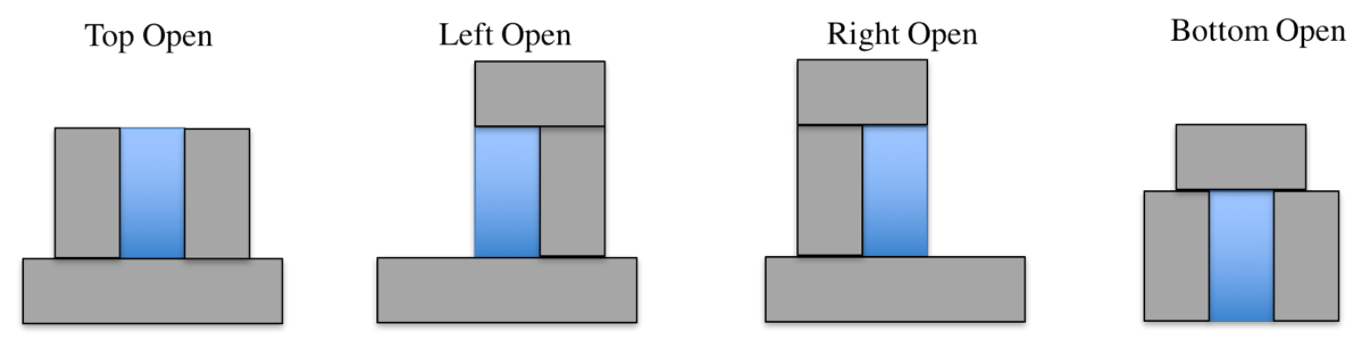

Note that this is from the view of the end of the detector without the PMT. An additional Pb Brick was used to shield the open end of the detector that is shown.

Fully shielded

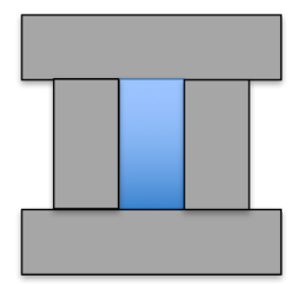

Bottom Open 3D drawing

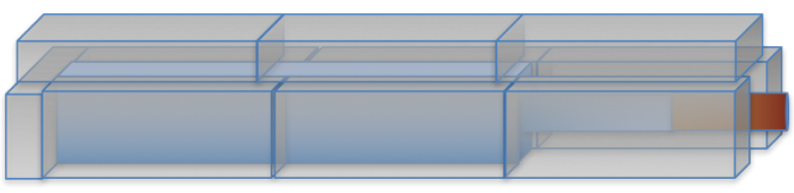

digiBase

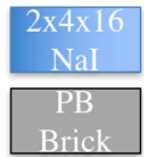

Figure 2.4.1. Configurations for the shielded NaI(TI) measurements.

Measurements were performed with each configuration for $15 \mathrm{~min}$., as shown in Figure 2.4.2, with the resulting count rates for each of the measurements shown in Table 2.4.1.

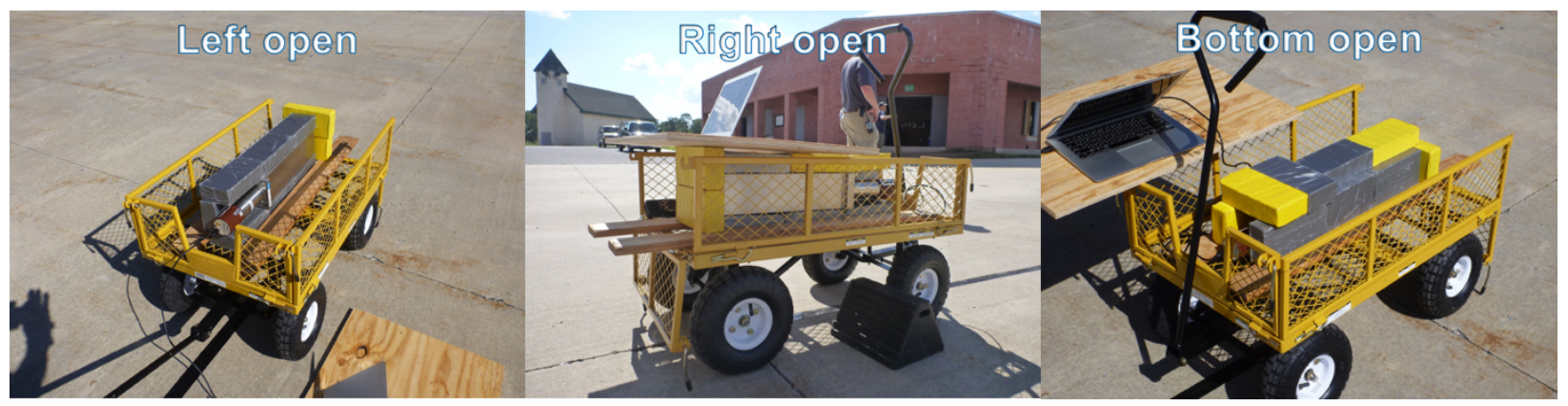

Figure 2.4.2. Measurement configuration examples for shielded directional NaI(TI) measurements. 
Table 2.4.1. Measurements with open face configurations and resulting count rates

\begin{tabular}{lc}
\hline Configuration & $\begin{array}{c}\text { Count rate } \\
\text { (cps) }\end{array}$ \\
\hline \hline Unshielded & 770.3 \\
Bottom open & 162.2 \\
Top open & 164.6 \\
Left open (toward open gravel at & 272.2 \\
$\quad$ garage) & \\
Right open (toward bar) & 296.8 \\
Tips open & 83.3 \\
\hline
\end{tabular}

The signal in the unshielded configuration is the sum of the radiation entering the crystal from each direction/face of the detector plus any intrinsic signal from detector noise (tube, etc.). The tips open configuration (i.e., bottom-right image in Figure 2.4.1) is a fair representation of the intrinsic signal from the detector, but with the tips open, it is expected to be higher than the intrinsic signal. The scatter from the lead could be included in the intrinsic signal of the shielded configurations that would not be present in the unshielded configuration.

Each open spectrum contains signal from both the open detector side and the intrinsic signal. If the open signal spectrum is summed, then the intrinsic signal is added each time, which skews the comparison with the fully unshielded configuration and will have the intrinsic component only one time. Therefore, the intrinsic signal should therefore be subtracted three times (except for the lead scattering, which should come out four times) for comparison with the unshielded configuration. A comparison of the resulting spectra from the wholly unshielded spectrum and the unshielded top, bottom, left, right, and tips is shown in Figure 2.4.3.

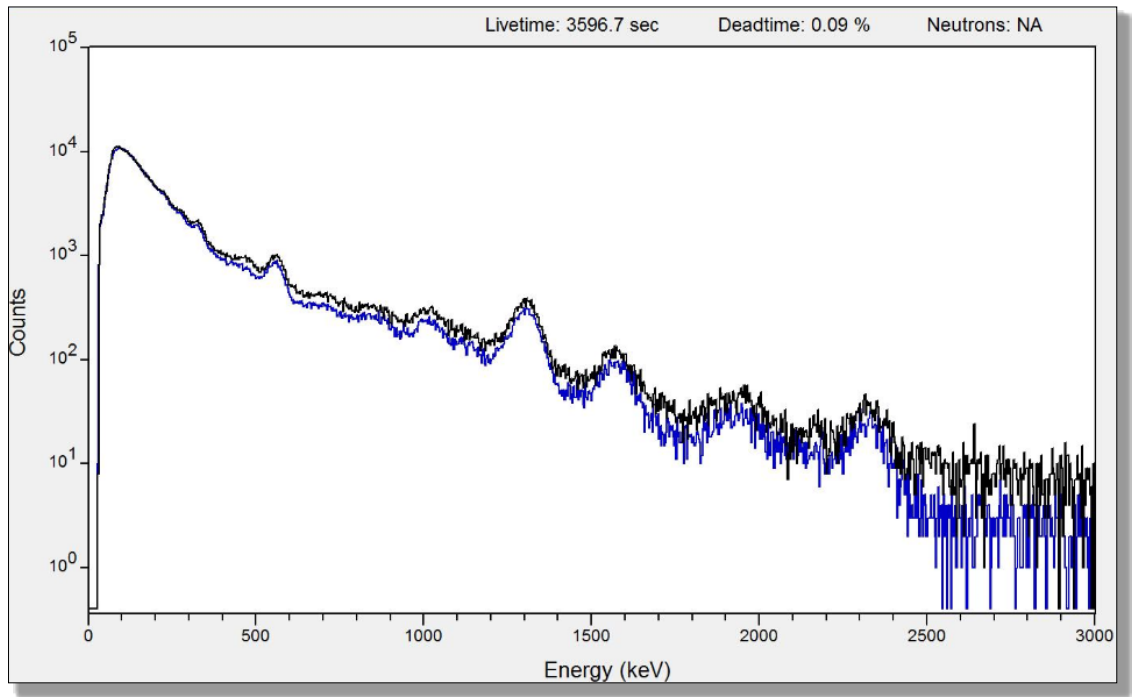

Figure 2.4.3. Comparison of the spectra from the wholly unshielded spectrum (black) and the unshielded top, bottom, left, right, and tip (blue).

If the tips are subtracted out two times, the comparison is shown in Figure 2.4.4. With the ends open, this could be close to the amount of intrinsic signal. There is very close agreement between the unshielded and summed components of the shielded configuration. 


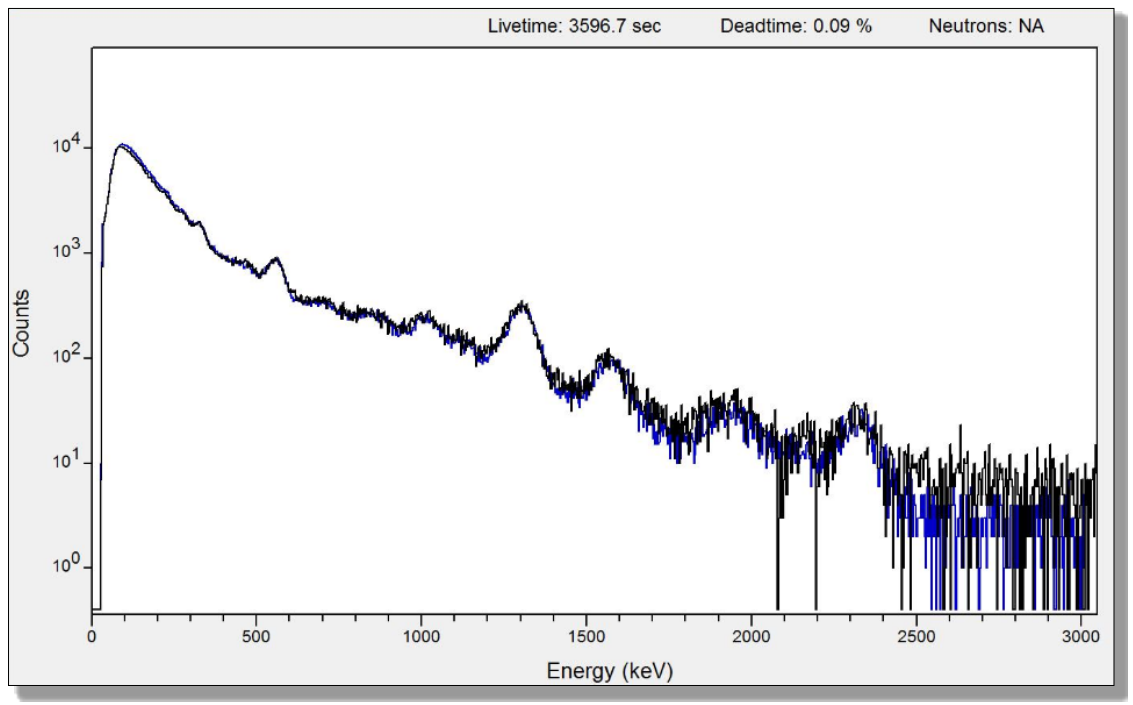

Figure 2.4.4. Comparison of the spectra from the wholly unshielded spectrum (black) and the unshielded top, bottom, left, right, and tip x 2 (blue).

From Figure 2.4.4, the sum of the signals measured from each detector face is in good agreement to the signal observed from the unshielded detector. This approach could therefore provide insight to the direction of the background contributing source at a location, but would require further investigation and testing to determine the precision and benefit of such results.

\subsection{STATIC NEUTRON MEASUREMENTS}

Static neutron background measurements using ${ }^{3} \mathrm{He}$-based ORNL detectors were performed along Main St. at the CACTF. The goal of the measurements performed with this system was to explore if additional neutron background measurements could potentially complement gamma-ray spectral measurements performed at FTIG to validate a radiation transport model. The equipment used to perform these measurements consisted of two separate PDT20A Neutron Pulse Monitoring Module ${ }^{3} \mathrm{He}$-based detector systems manufactured by Precision Data Technology Inc. Each module contained of a square panel of four $30 \mathrm{in.} \mathrm{long}{ }^{3} \mathrm{He}$ (atmosphere pressurized) fully surrounded with polyethylene. Upon neutron detection in any of ${ }^{3} \mathrm{He}$ tubes per panel, a positive (TTL) signal was sent to the data acquisition system and recorded as a gross count from the detector panel.

Measurements were performed along Main St. ( $x$ axis) in approximately $5 \mathrm{~m}$ increments with dwell times of 15 min. per location. Figure 2.5.1 shows the measurements of total neutron counts per panel per second at each position for both panels. 


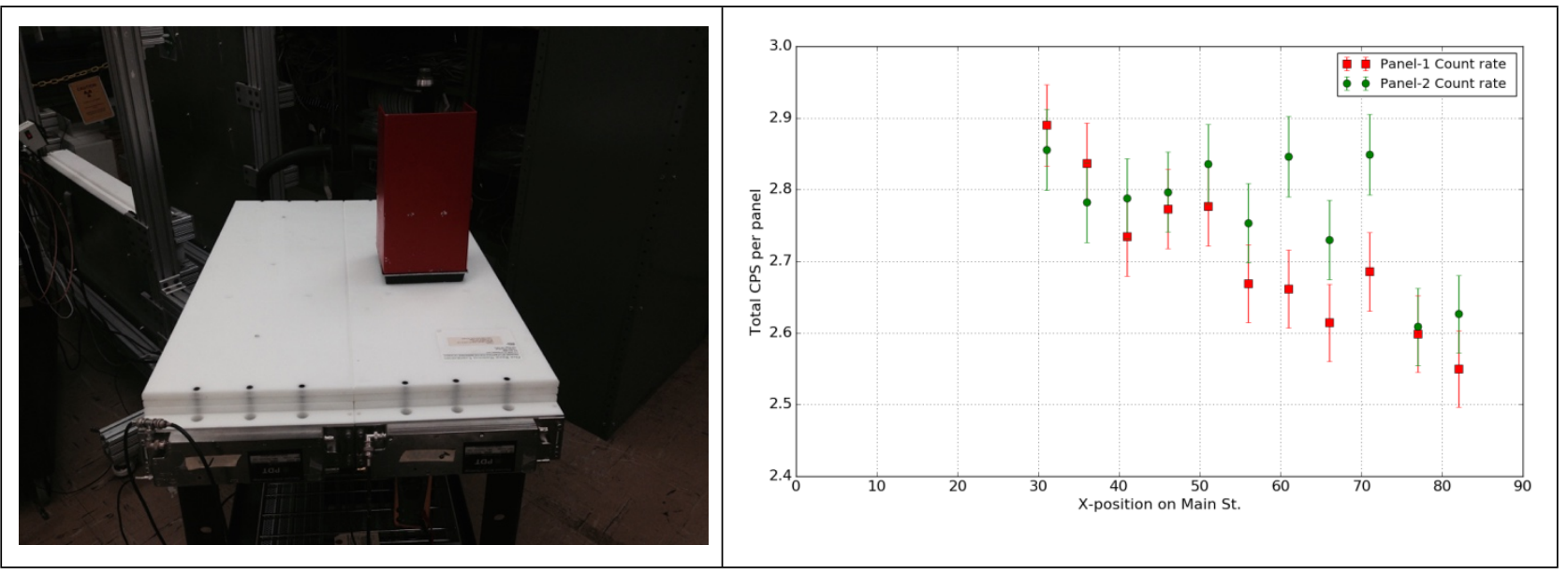

Figure 2.5.1. Static neutron detection system measurements along Main St. (Left) Photograph of ${ }^{3}$ He detection system. (Right) Total neutron counts per panel per second versus position on Main St.

A downward trend in detected neutron counts per second can be observed for both panels as the position along the $x$ axis increases. Although small, the decrease in detected neutron counts is likely a result of the presence of taller buildings, such as the hotel, and lower relative street heights at various points along the $x$ axis. These buildings shield more sky, therefore shielding more cosmic radiation from the detector system and causing a suppression in the observed background counts [1]. Further measurements and analysis of neutron data will be needed to help guide and verify future neutron transport integration into the particle transport testbed.

\subsection{GEOREFERENCING}

Similar DGPS measurements from OPTUS 1 were also performed during OPTUS 2. The $x$ axis for this measurement campaign went from $(0,0)$ to $(84,0)$, with DGPS measurements taken every $5 \mathrm{~m}$. The $y$ axis went from $(0,23)$ to $(0,-22)$, and DGPS measurements were also collected every $5 \mathrm{~m}$ increment. These measurements were performed as a cross check to verify the points measured during OPTUS 1 .

Measurements were also taken on the $x$ axis at the $17.42 \mathrm{~m}, 28.43 \mathrm{~m}, 39.59 \mathrm{~m}, 54.6 \mathrm{~m}$ and $66.75 \mathrm{~m}$ marks at the $4.5 \mathrm{~m}, 6.75 \mathrm{~m}, 9.0 \mathrm{~m},-4.5 \mathrm{~m},-6.75 \mathrm{~m}$, and $-9.0 \mathrm{~m}$ points. Measurements were also taken on the $y$ axis at the $20 \mathrm{~m}, 10 \mathrm{~m}, 0 \mathrm{~m},-10 \mathrm{~m}$, and marks at the at the $4.5 \mathrm{~m}, 6.75 \mathrm{~m}, 9.0 \mathrm{~m},-4.5 \mathrm{~m},-6.75 \mathrm{~m}$, and $-9.0 \mathrm{~m}$ points. These measurements were performed to georeference the locations where neutron ship cookie measurements were performed by Remote Sensing Laboratory (RSL). The locations of both persistent meteorology stations and neutron ship cookie calibration measurement locations (near roundabout) were also recorded. All the DGPS measurement points are shown in Figure 2.6.1. 


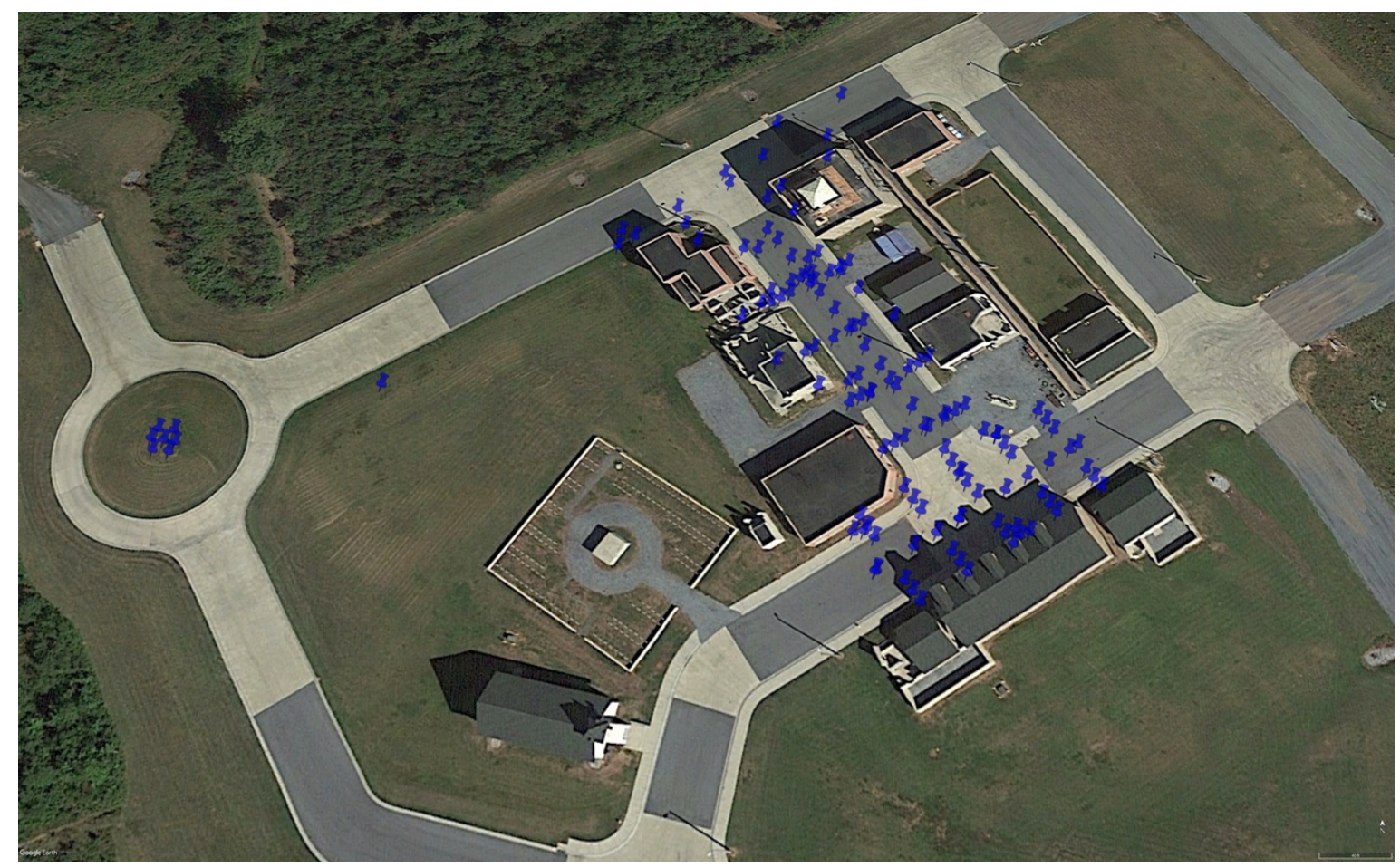

Figure 2.6.1. DGPS points for OPTUS 2 measurements.

\subsection{LIDAR MAPPING OF CACTF BUILDING INTERIORS}

The LIDAR data taken during OPTUS 2 focused on capturing the interior and exterior of the hotel building at FTIG. A total of 78 scans were conducted throughout the hotel using the same long-dwell high-fidelity Z+F Imager system as used in OPTUS 1. The individual scans were registered (i.e., stitched together) based on target overlap into a single comprehensive point cloud for each floor of the interior and the exterior of the hotel (Figure 2.7.1).

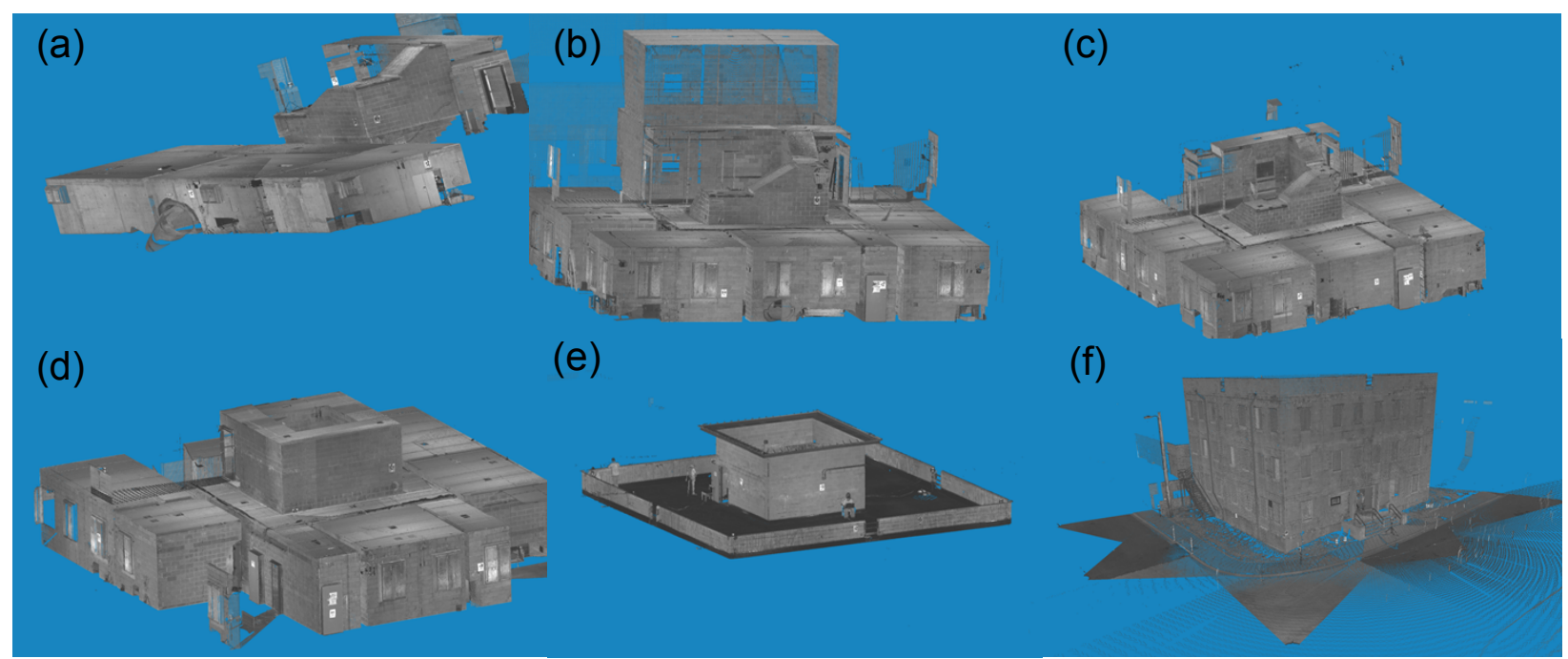

Figure 2.7.1. LIDAR-generated point clouds for the hotel on CACTF. (a) Basement, (b) First floor, (c) Second floor, (d) Third floor, (e) Roof, and (f) Hotel exterior. 
These point cloud models were then further stitched together to generate completed combined scan creating a full exterior and interior 3-D point cloud of the hotel (Figure 2.7.2).

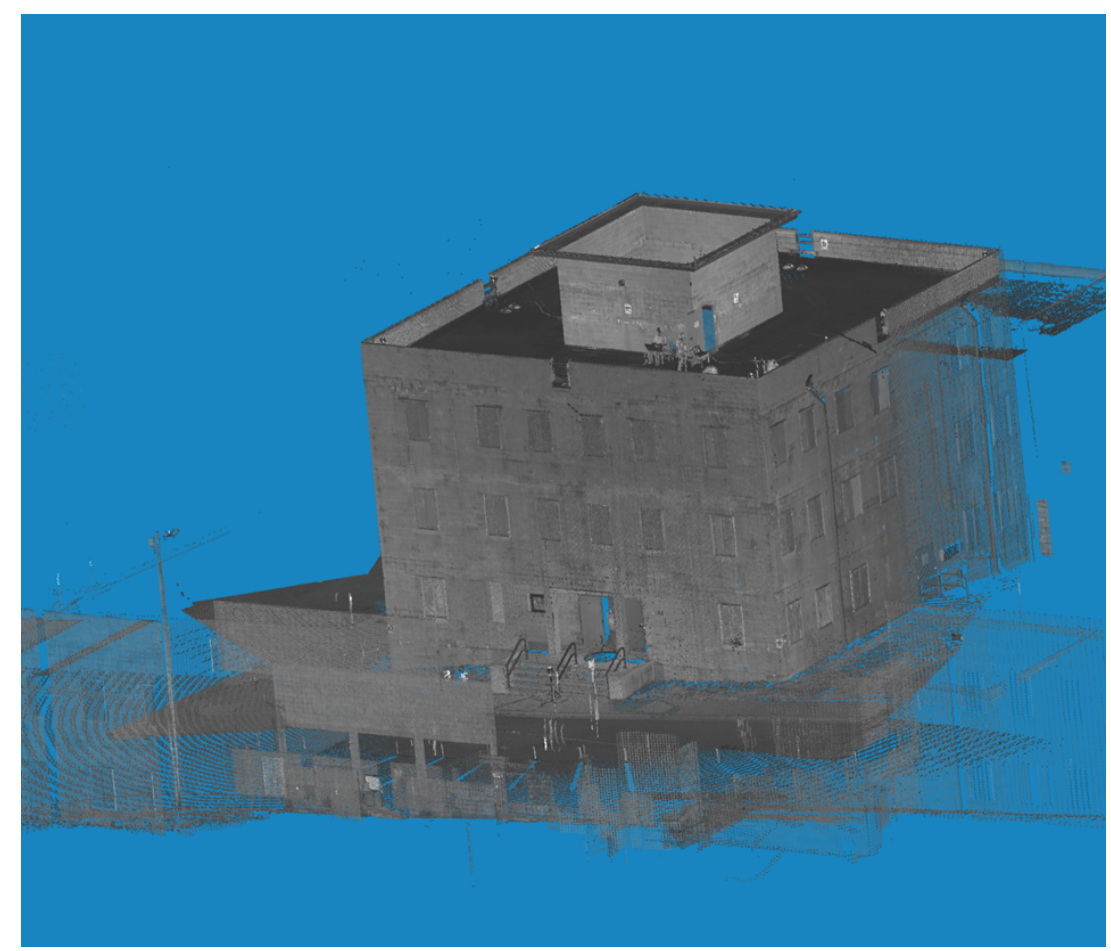

Figure 2.7.2. Combined LIDAR point cloud of interior and exterior of hotel.

This point cloud will serve as ground truth for comparison with MUSE 1 imagery taken with ORNL pedestrian LIDAR system. In addition, the analysis demonstrated fusion of the point cloud of the hotel with the point cloud of FTIG taken at the previous campaign. While proof-of-principle extraction of CAD elements using the same manual method used to generate the FTIG-wide CAD was confirmed, no comprehensive CAD model of the interior of the hotel has been built to date.

\subsection{METEOROLOGICAL AND PERSISTENT RADIATION MONITORING}

Two separate meteorological and persistent radiation monitoring systems were used during the OPTUS 2 campaign that were identical to the systems used during OPTUS 1. As with OPTUS 1, the goal of these measurements was to compliment the measurement of gamma-ray backgrounds at the CACTF by monitoring area radiation with a persistent detector system and using it as a reference for anomalies. The second station was moved from the ROC to the CACTF near the gas pumps to provide immediate local weather for both the east and west areas of Range 30. This data was also recorded to study potential weather and environment-related effects on measurements of natural gamma-ray background spectra and establish systematic uncertainties caused by them.

Data collected by the RSI NaI(Tl) detector that was placed by the FTIG roundabout next to Davis 2 weather system included five large (each $\sim 250 \mathrm{MB}$ ) .csv files. Each line inside the .csv file corresponds to approximately $1 \mathrm{~s}$ of recorded data and provides raw counts per second distributed between 1,023 bins from $(0,1023)$, equivalent to 10-bit ADC. As in the OPTUS 1 campaign analysis, spectra were added second-by-second for $5 \mathrm{~min}$. intervals and then correlated to the corresponding weather file readings, which reports weather parameters every $5 \mathrm{~min}$. Using the recorded times from each unit, the data was correlated and combined in ROOT TTree structure format for further analysis. 
For the OPTUS 2 campaign, there were serious issues with the output .csv files because of the data storage and extraction process with the RSI-700 system database. Like OPTUS 1, data had significant time overlap and duplication between recorded data, but the OPTUS 2 data had a large portion of timeline missing from extracted radiation measurements from the RSI. Figure 2.8.1 shows the RSI detector gross counts and ROI counts per 5 min. versus the time overlaid with time-equivalent temperature readings from the Davis Pro 2 weather station.

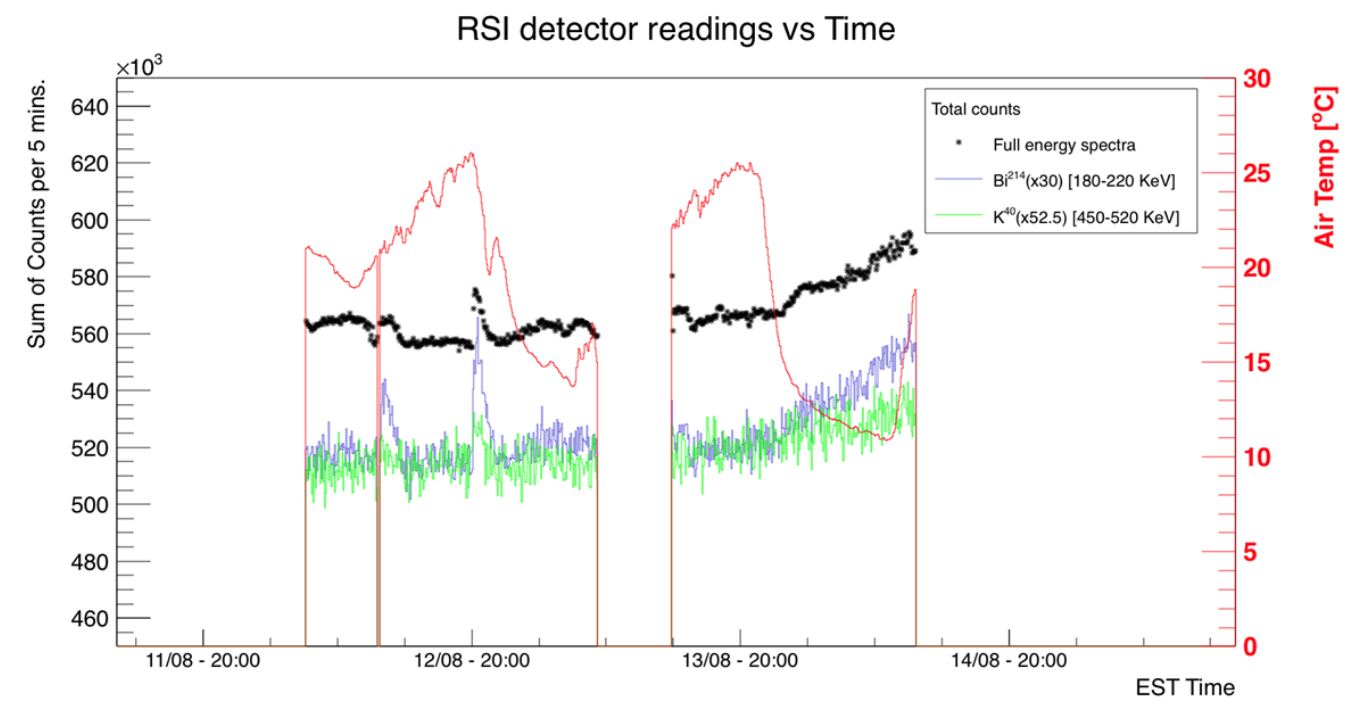

Figure 2.8.1. Detector response with two relevant ROI 5 min. counts compared to air temperature.

As shown in Figure 2.8.1, approximately only $48 \mathrm{~h}$ of radiation data was available for analysis of weather correlation. Additionally, several hours of data plotted here cannot be used because of the spectral distortion caused by ${ }^{137} \mathrm{Cs}$ movements around the range. No rain events were recorded, so there was no immediate correlation determined. However, the limited dataset will be included in the data library being compiled for correlated weather and radiation measurements.

Ideally, the persistent weather and radiation data would be collected in a single correlated database that has the various components synced during recording for easier and more accurate post-processing. After OPTUS 2, a revised persistent radiation system was developed at ORNL utilizing a 2 in. $\times 4$ in. $\times 16$ in. $\mathrm{NaI}(\mathrm{Tl})$ detector and Davis Pro 2 weather station. Both of these components, along with other environmental sensors, were later integrated with the data acquisition system used to collect the static $\mathrm{NaI}(\mathrm{Tl})$ measurements collected at FTIG. 


\section{OPTUS 3 MEASUREMENT CAMPAIGN}

The third data collection, OPTUS 3, was performed at FTIG in November 2015. This data collection was performed solely by the RSL team and aimed to collect NaI(Tl) measurements along Main St. at the CACTF using a 2 in. $\times 4$ in. $\times 16$ in. Radiation Solutions Inc. (RSI) system. This data was used to validate the virtual testbed created using geometry measurements and source terms derived from HPGe measurements taken at OPTUS 1 and 2.

\subsection{RSI NaI(TI) MEASUREMENTS}

\subsection{1 "Basic" Measurements}

At early OPTUS meetings with the sponsor and the advisory board, the emphasis on generating a tool that could assist in clearing building interiors was reduced in favor of supporting the planning of wide-area exterior (WAE) searches and intelligence-driven searches of specific buildings. For validation of the transport codes, measurements should be taken that include attenuation of exterior wall. Measurements with both source and detectors inside the CACTF Hotel were made during OPTUS 2, but no detector locations outside the hotel were used.

Typical construction practices do not fill cinderblocks. Measurements were made with a source and detector placed on opposite sides of a wall to verify that the walls are not filled with cement mortar. The positions (1) aligned with the center of a void space and (2) aligned with a central rib are shown in Figure 3.1.1. If these two measurements are similar, then the blocks are filled. If very dissimilar, the blocks are not filled. In addition to the source measurements, measurements in the same locations were made with the source removed for background correction/subtraction. These measurements were also intended to provide a check on the overall density of the wall materials used in the models, which is also important for the scatter contribution in the wide-area search measurements.
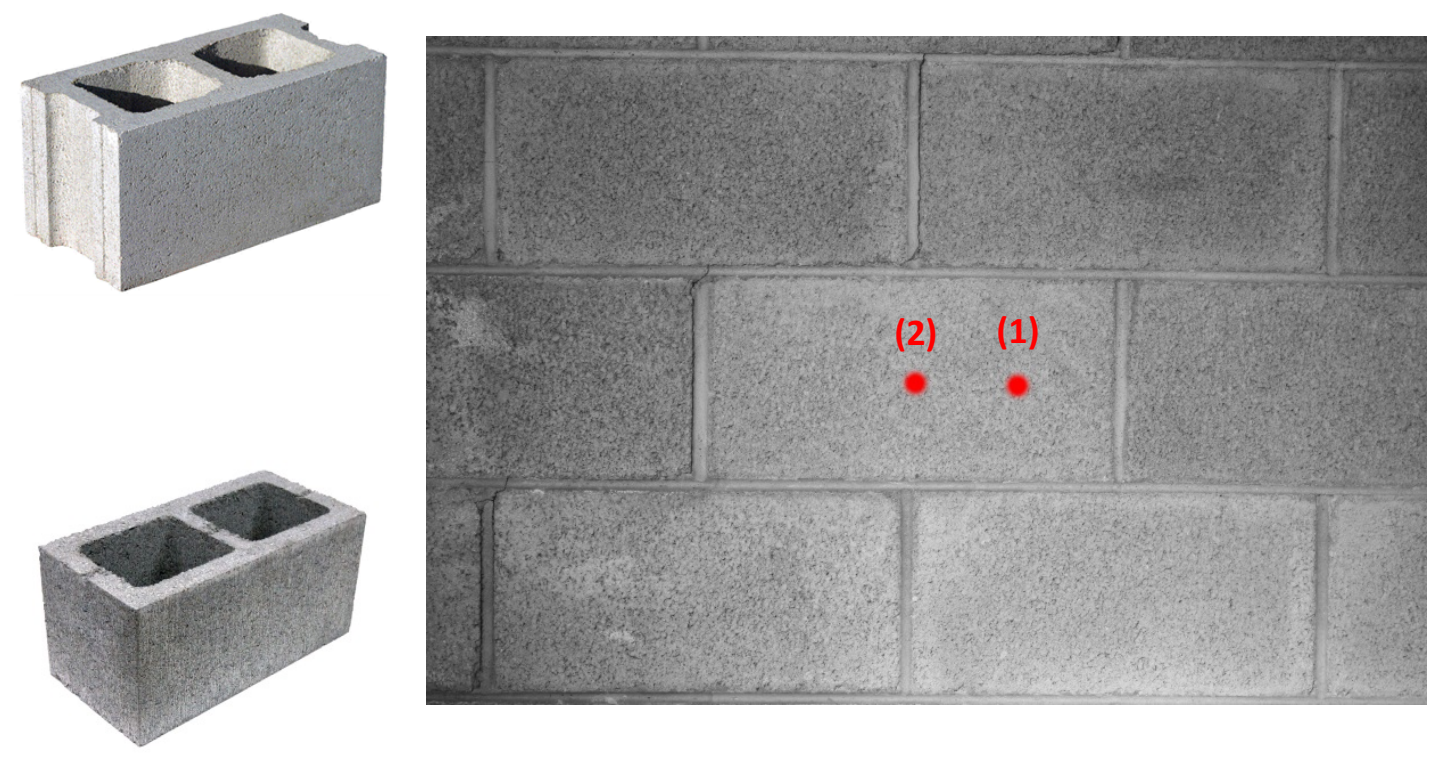

Figure 3.1.1. Example cinder block construction and measurement target locations. 
A total of six cinderblocks were measured at locations throughout the site. Care was taken to sample a wide a variety of inferred material compositions based on observation of the color of the blocks and their relative locations in the structures. Additionally, the team chose locations that were as uniform as possible in terms of the number and composition of adjacent blocks.
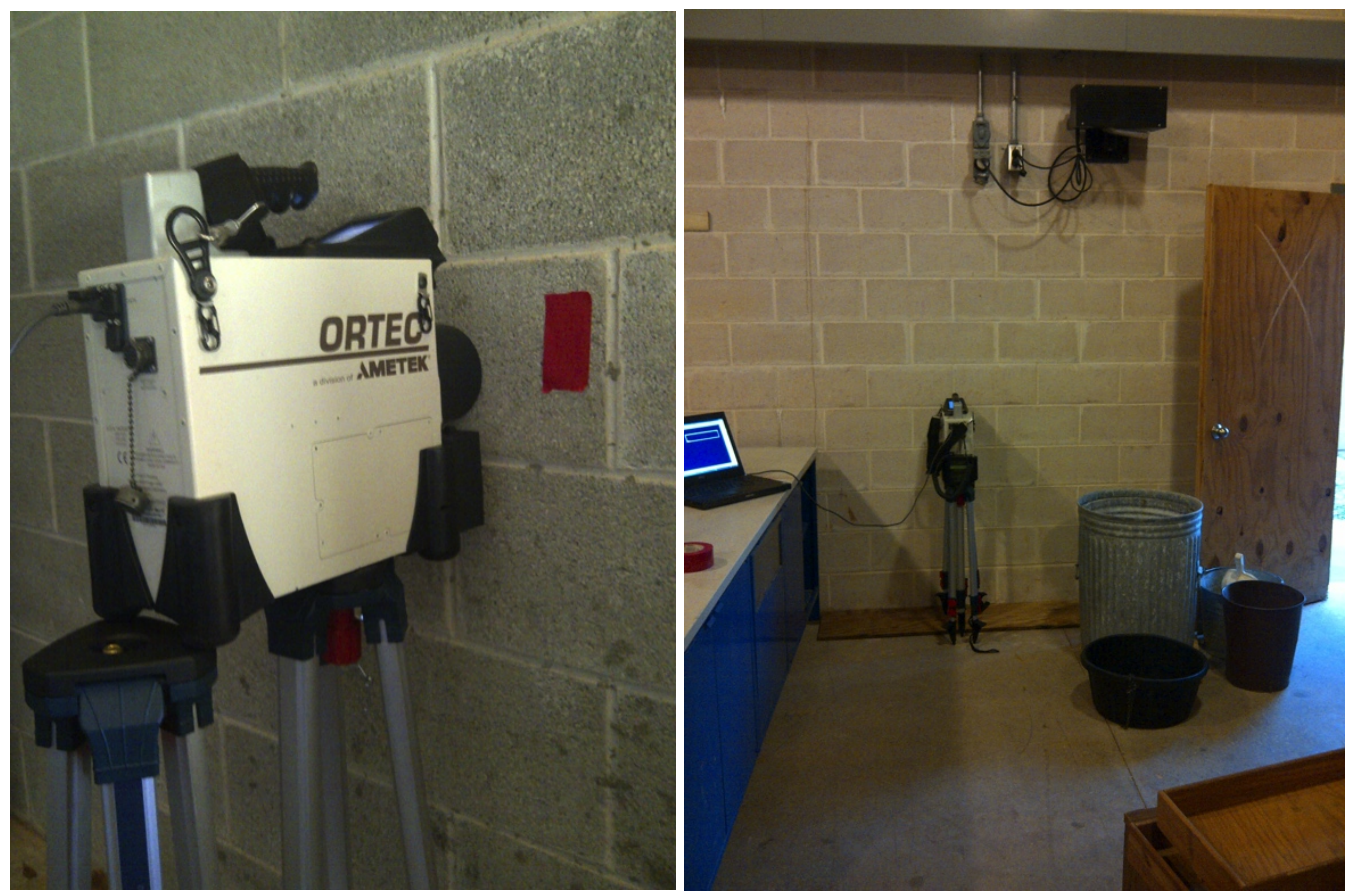

Figure 3.1.2. Illustration of cinder block measurements.

Optimal locations were typically far from building corners, windows, doors, and expansion joints. Two background and two source measurements were performed for a total of 24 basic measurements. For simplicity's sake, each measurement duration was set to $5 \mathrm{~min}$., which provided ample statistics for analysis. The source used was a $\sim 1 \mu \mathrm{Ci}{ }^{137} \mathrm{Cs}$ sealed-disk check source. A mechanically cooled RSL AMETEK Detective 100 was used for the basic measurements.

\subsubsection{Wide-area Exterior Measurements}

The purpose of the WAE measurements is to provide direct validation of the transport model to recreate measurements with sources present. These measurements, taken down the center of the road, also comport with the kinds of data that would be collected during routine radiological/nuclear search and route-clearing operations. The geometry of the WAE measurements is shown in the analysis section below. Data were collected at a total of 17 points using a pair of RSI RSX-1 detector modules. The coordinate system defined for the previous measurement sets was used. The detectors were placed on folding tables, which were given fiducial marks to ensure placement consistency among measurements. A total of 18 locations were measured. At each location, a $10 \mathrm{~min}$. background spectrum was collected, as well as a $10 \mathrm{~min}$. spectrum with the source in place. The source used was a collection of ${ }^{137} \mathrm{Cs}$ sealed-disk check sources with an aggregate activity of $81 \mu \mathrm{Ci}$. The data were recorded on laptop computers running the RSI RadAssist software. The data packages output from RadAssist contain all of the pertinent detector calibration and scaling parameters for troubleshooting. 


\subsubsection{High Interest Locations}

One of the missions CONOPS identified targeted by the OPTUS development team is the so-called highinterest location. Such a location could be the result of an intelligence-driven mission or may be indicated by a weak or indeterminate anomaly detection. The purpose of the high-interest location measurements was to produce measurements that model the collection of data from the street with a source positioned within a structure. The idea is that the OPTUS tool could potentially be used to optimize the geometry of a long-dwell measurement or the speed and approach of a transient one.

For this measurement set, a $40 \mathrm{mCi}{ }^{137} \mathrm{Cs}$ source was placed in front of the hotel elevator doors as indicated in Figure 3.1.3. Data were collected at each of the seven locations indicated with the source on the floor of the building as well as with the source elevated $1 \mathrm{~m}$ above the floor. Background data were collected for each geometry to account for any potential temporal variation of the background radiation field. A total of 28 measurements were made. The detectors used and their setup was identical to that used in the WAE measurements with 10 min. collection times.

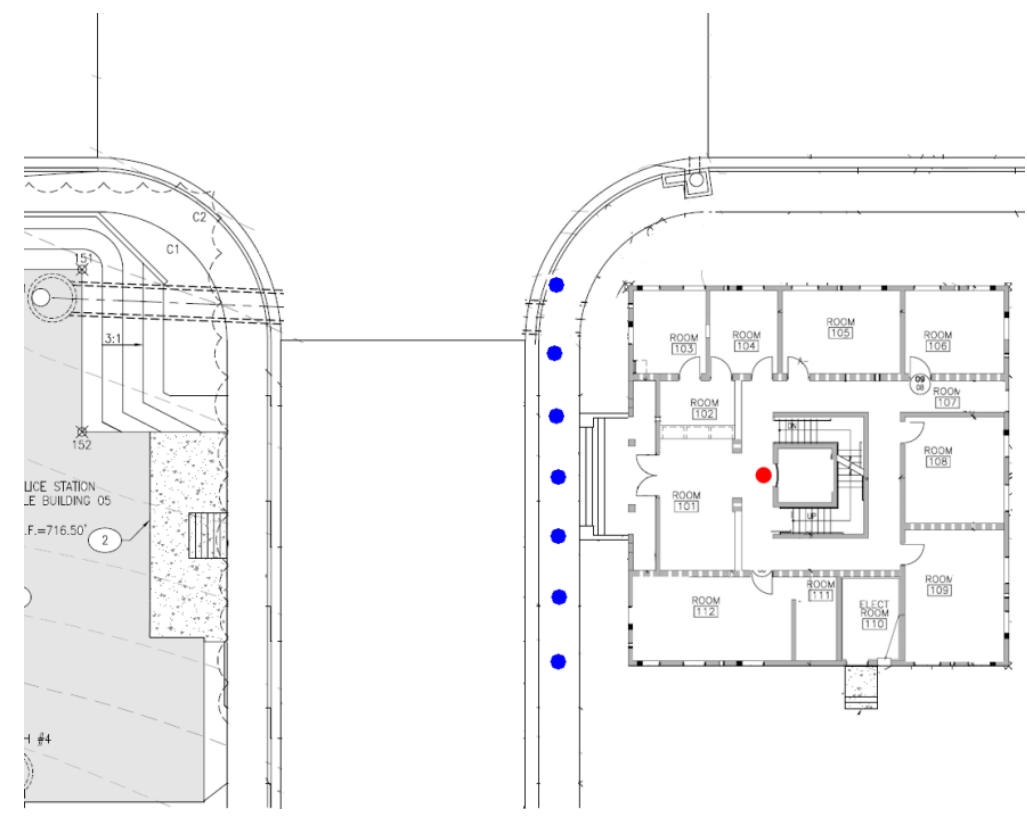

Figure 3.1.3. The high-interest location measurement geometry.

\subsection{MODELING COMPARISONS TO MEASUREMENTS}

Modeling was done for a subset of the measurements discussed above. Modeling was focused on the WAE measurements, using standard $\mathrm{NaI}(\mathrm{Tl})$ detectors in the main roadway with an $81 \mu \mathrm{Ci}$ cesium source placed in between buildings. Four source locations were used with several detector locations for each source location. For some combinations of source and detector positions, there was a direct line-of-sight between the two. In other cases, the direct line-of-sight was blocked by buildings. Distances between the source and detector varied between 12 and $35 \mathrm{~m}$. The source and detector locations are listed in Table 3.2.1 and shown in Figure 3.2.1. In Table 3.2.1, detector locations in boldface have a direct line-of-sight view of the source and locations in red had no data recorded. A photograph of two detector locations $(x=30 \mathrm{~m}$ and $x=40 \mathrm{~m})$ is provided in Figure 3.2.2. Example spectra are shown in Figure 3.2.3 with and without the cesium source present. 
Table 3.2.1. Source and detector locations $($ Bold $=$ direct line-of-sight to source $\mid$ Red $=$ no data recorded)

\begin{tabular}{l|ccc}
\hline Measurement & $\begin{array}{c}\text { Gravel area } \\
\text { between }\end{array}$ & $\begin{array}{c}\text { Distance from } \\
\text { road centerline } \\
(\mathbf{m})\end{array}$ & $\begin{array}{c}\text { Road centerline } \\
\text { detector locations } \\
(\mathbf{m})\end{array}$ \\
\hline \hline WAE 1 & Bar and office & 16.5 & $10, \mathbf{2 0}, \mathbf{3 0}, \mathbf{4 0}, 50$ \\
WAE 2 & Bar and office & 30.0 & $10,20,30,40$ \\
WAE 3 & Hotel and OPS & 11.0 & $\mathbf{3 0}, \mathbf{4 0}, 50,70, \mathbf{8 0}$ \\
WAE 4 & Hotel and OPS & 16.5 & $\mathbf{3 0}, \mathbf{4 0}, \mathbf{5 0}, \mathbf{7 0}, 80$ \\
\hline
\end{tabular}

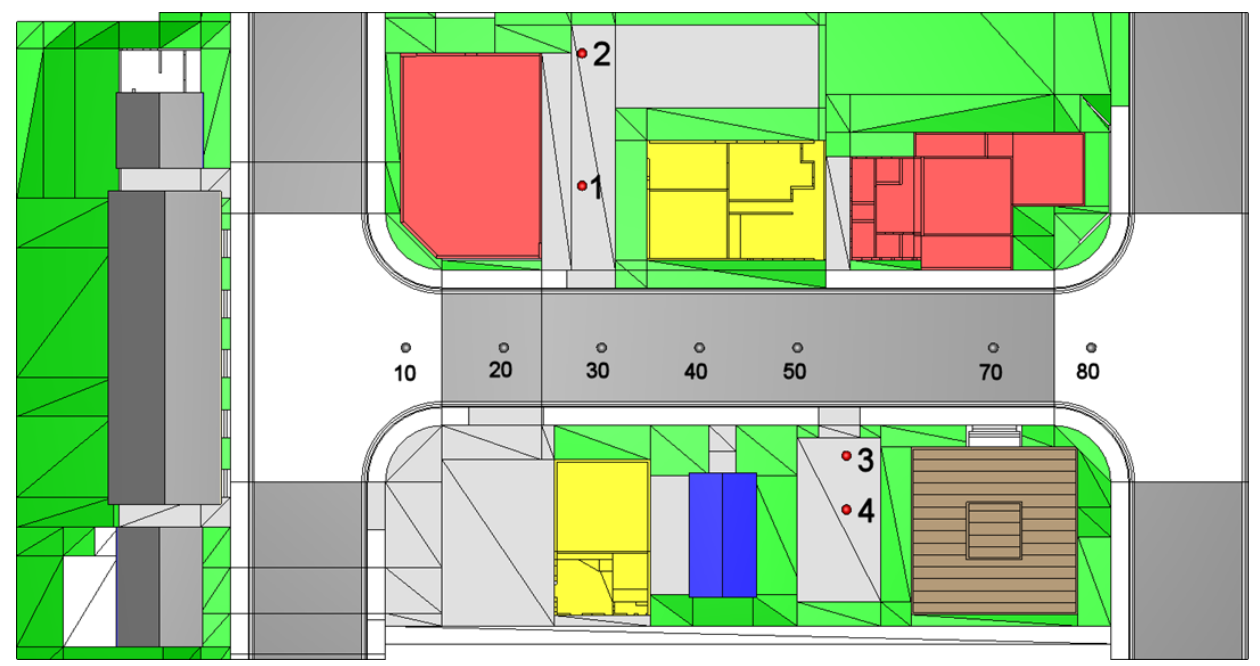

Figure 3.2.1. Overhead view of the main street of the FTIG CACTF showing the source locations (red circles, labeled 1-4) and detector locations, in meters (gray circles, labeled 10-80). 


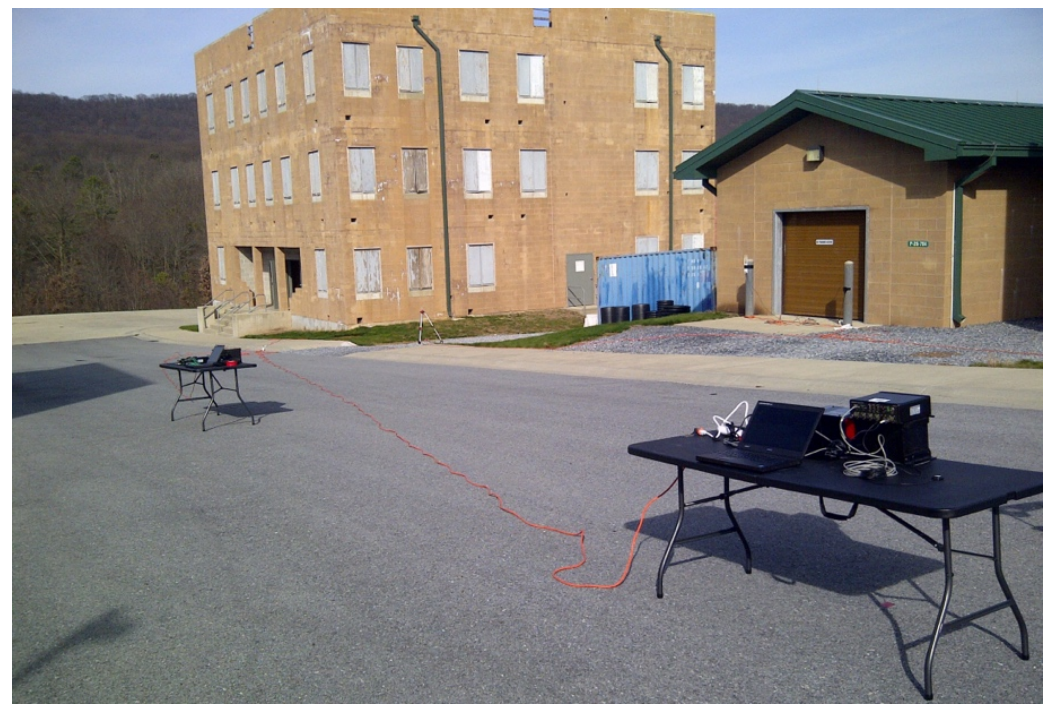

Figure 3.2.2. Photograph of two detector systems outside high-interest location.
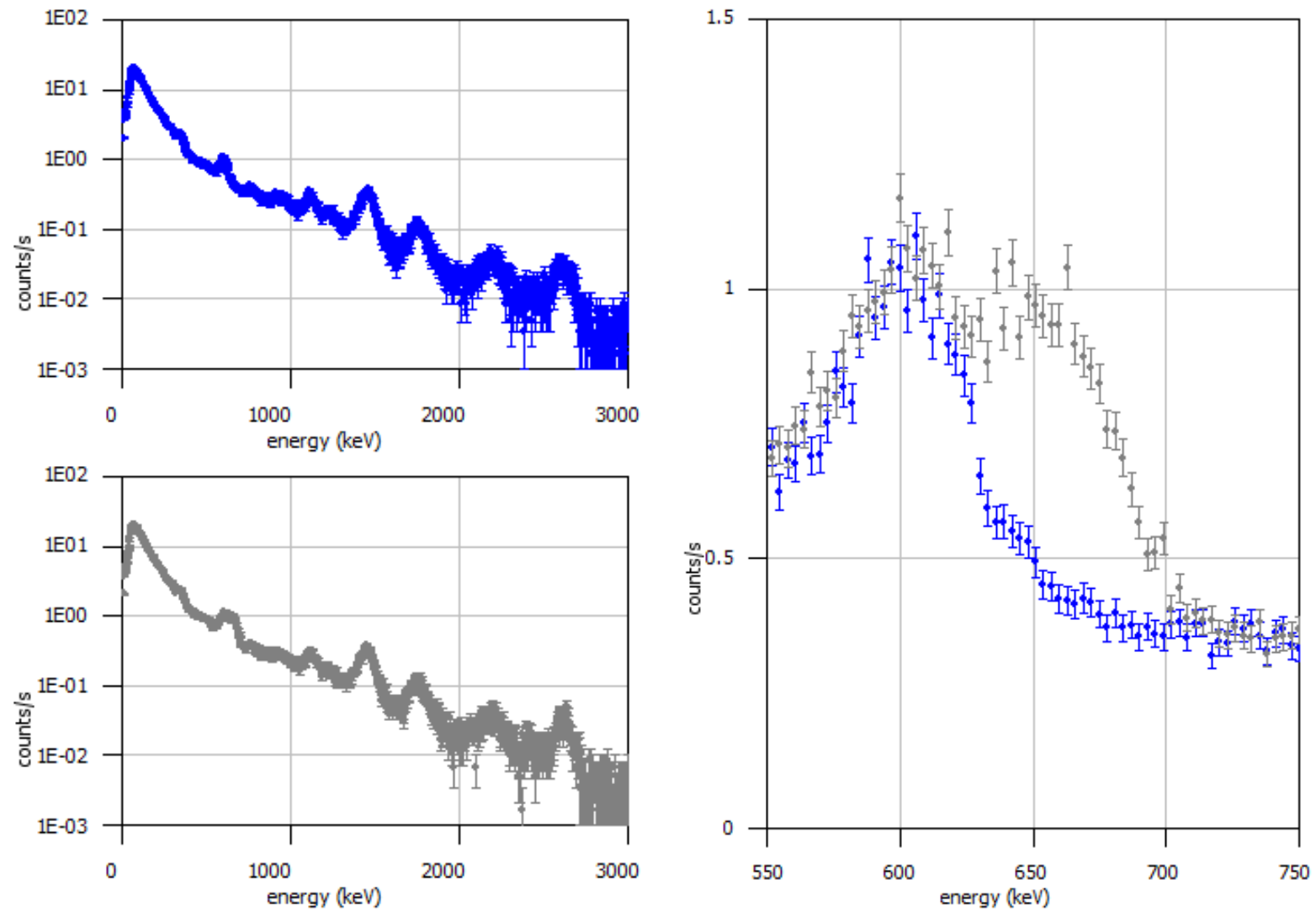

Figure 3.2.3. Measured spectra for detector location $x=30 \mathrm{~m}$. Background only (blue, upper left), with the cesium source (gray, lower left) and both spectra near the $662 \mathrm{keV}$ region (right). 


\subsubsection{Simulation Model}

A 3-D computational model of the FTIG CACTF for use in the SCALE/MAVRIC [2][3] radiation transport package was developed from the construction drawings supplied by the US National Guard. The model includes the area and buildings near Main St. If future measurements are made at other areas in the site, the model can be expanded. The current model includes four concrete intersections, six asphalt roads, a gravel road, sidewalks, curbs, soil with a rough approximation of terrain elevation, and nine buildings. Eight of buildings consist mostly of just their outer shells, but the hotel includes interior floors and walls because measurements were made inside that building during the OPTUS 1 and OPTUS 2 campaigns. The size of the model is $416 \mathrm{ft} \times 409 \mathrm{ft} \times 66.667 \mathrm{ft}(126.8 \mathrm{~m} \times 124.7 \mathrm{~m} \times 20.3 \mathrm{~m})$. The coordinates in the model are consistent with the measurements; that is, the origin is located where the centerline of Main St. intersects the centerline of the street in front of the townhouses. The $x$ axis runs along the centerline of Main St., and the $y$ axis runs along the centerline of the street in front of the townhouses. The model geometry is shown in Figure 3.2.4.

Materials in the model were assigned based on information in the construction drawings. Elemental composition and density data for each material were taken from standard materials listed in a Pacific Northwest National Laboratory report [4] for initial calculations while KUT NORM analysis was being performed.

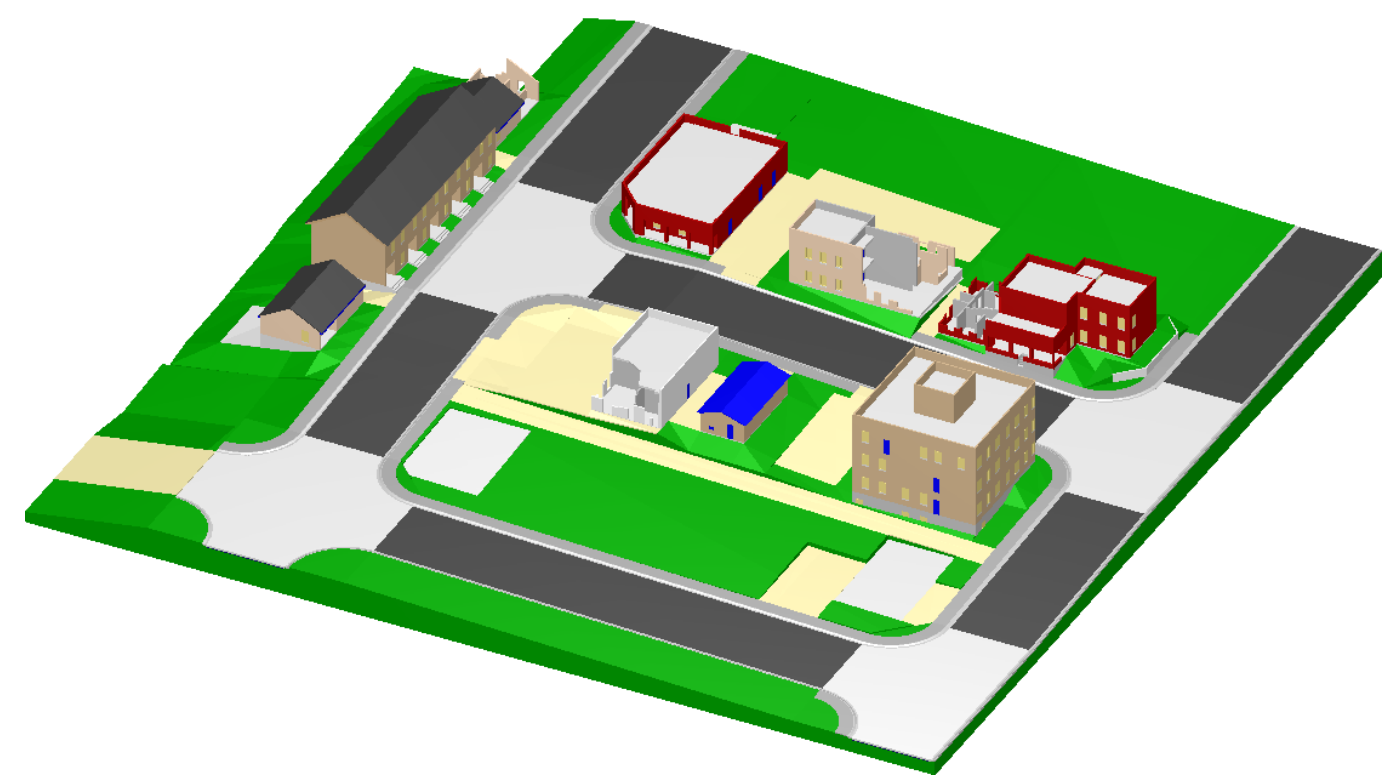

Figure 3.2.4. The full FTIG CACTF model with nine buildings, concrete intersections (white), asphalt roads (black), gravel areas (tan), and soil (green).

\subsubsection{NORM Concentration Values}

For photons in urban areas, nearly all the background is caused by the presence of NORM in the soil, roadways, and buildings. The main contributors to NORM are ${ }^{40} \mathrm{~K},{ }^{232} \mathrm{Th}$, and its daughter products and ${ }^{238} \mathrm{U} / 235 \mathrm{U}$, as well as their daughters. The proportion of background from the atmosphere (from space or radon brought in with the wind) is usually small. To model the background at the FTIG CACTF site, detailed measurements on the surfaces of the different materials of the site were taken, and the concentrations of the NORM components were derived [5], which were discussed previously with the OPTUS 1 and OPTUS 2 campaigns. The values are listed in Table 3.2.2. The geometry and materials 
under the curbs were not well known, making the determination of the concentrations difficult. Values for the curb concrete are assumed to be the average of the intersection concrete and the sidewalk concrete. There are a few other uncertainties (e.g., actual gravel depths, density, gaps) that need to be evaluated to see what impact they would have on the final values.

Table 3.2.2. Concentration of NORM for the major materials at the FTIG CACTF

\begin{tabular}{lrrrr}
\hline \multirow{1}{*}{\multicolumn{1}{c}{ Material }} & \multicolumn{4}{c}{$\begin{array}{c}\text { Concentration } \\
(\mathbf{B q} / \mathbf{k g})\end{array}$} \\
\cline { 2 - 5 } & \multicolumn{1}{c}{${ }^{\mathbf{4 0}} \mathbf{K}$} & ${ }^{{ }^{332} \mathbf{T h}}$ & ${ }^{238} \mathbf{U}$ & ${ }^{137} \mathbf{C s}$ \\
\hline \hline Asphalt & 97.54 & 3.96 & 24.34 & 0.00 \\
Cinder block, dark tan & 156.98 & 10.82 & 11.78 & 0.00 \\
Cinder block, gray & 200.05 & 7.90 & 14.07 & 0.00 \\
Cinder block, red & 192.85 & 12.17 & 13.51 & 0.00 \\
Cinder block, tan & 318.62 & 13.25 & 15.75 & 0.00 \\
Cinder block, white & 112.81 & 7.25 & 9.89 & 0.00 \\
Concrete, curb & 236.27 & 10.33 & 17.93 & 0.00 \\
Concrete, foundation & 190.17 & 9.22 & 14.81 & 0.00 \\
Concrete, intersection & 231.26 & 10.20 & 18.21 & 0.00 \\
Concrete, patio & 219.30 & 9.63 & 18.22 & 0.00 \\
Concrete, sidewalk & 241.28 & 10.45 & 17.64 & 0.00 \\
Concrete, stairs & 204.52 & 8.68 & 17.46 & 0.00 \\
Gravel & 51.00 & 3.58 & 23.02 & 0.00 \\
Soil & 412.63 & 37.75 & 25.88 & 5.74 \\
\hline
\end{tabular}

\subsubsection{Background Source Descriptions}

The energy spectra for the NORM components were computed using SCALE/ORIGEN ${ }^{4}$ [6] to accurately account for all the daughter products. The calculation assumed that the daughter products were all in transient equilibrium with the parent, which may not always be true. Radon is present in both the thorium and uranium decay sequences, and because it is a gas, it could escape from the material before it decays into the next isotope in the decay sequence. Materials processing techniques could alter the elemental ratios (called technologically enhanced NORM).

In the process of determining the NORM concentration values for the FTIG CACTF materials, the concentrations of several individual isotopes in each decay chain were determined and were consistent with the transient equilibrium assumption. If the elements below radon were at lower activities than the elements above radon, the chains could have been split into two subchains each with separate concentration values, but this split was not necessary.

Typical radiation transport codes require the user to define the geometric extent and strength of each source. The volume of the source is required to determine the strength. Because the materials with NORM sources have very large extents with difficult-to-compute volumes (soil) or consist of many small bodies (cinder block walls of buildings), a more automated approach was taken in constructing source descriptions. A $416 \times 409 \times 66$ mesh $\left(1 \mathrm{ft}^{3}\right.$ voxels $)$ was overlaid across the geometry model, and the

\footnotetext{
${ }^{4}$ SCALE is a comprehensive modeling and simulation suite for nuclear safety analysis and design developed and maintained by Oak Ridge National Laboratory. Within SCALE, the ORIGEN (Oak Ridge Isotope Generation) code calculates time-dependent concentrations, activities, and radiation source terms for radiation isotopes.
} 
fraction of each real material within each voxel was determined. With these values, the total volume of each material across the site could be found, and the total strength determined. A set of mesh-based sources, one for each material or NORM component combination, was created.

Biasing factors were applied to each of the mesh-based sources so that when used in a Monte Carlo calculation, more photons would be sampled nearest the centerline of Main St. To preserve a fair Monte Carlo game, photons sampled closer to the detector locations were weighted lower than photons sampled farther from the detectors.

\subsubsection{Monte Carlo Calculations}

A common way to efficiently simulate detector systems is to break the simulation into two steps:

(1) transport photons from the various sources to the location of the detector and determine the energydependent flux and (2) transport photons within the detector to compute the energy deposited, which goes into making the pulse-height distribution. Note that the detector is not modeled in the first step so that the same transport calculation for step 1 can be used with several different types of detectors in step 2 . The calculations in step 2 can be done once for each type of detector of interest - each is called a detector response function. After running step 1, the energy-dependent fluxes from anywhere in the transport model can be folded with one or more detector response functions, which is much more efficient than explicitly modeling individual detectors in a site-wide transport calculation.

For this project, three detector response functions were created for a 2 in. $\times 4$ in. $\times 16$ in. $\mathrm{NaI}(\mathrm{Tl})$ crystal surrounded by $1 \mathrm{~mm}$ of aluminum using Monte Carlo Neutral Particle (MCNP) [7]. Photons incident perpendicularly to the 2 in. $\times 4$ in. face (orientation 1), the 2 in. $\times 16$ in. face (orientation 2 ) and the $4 \mathrm{in}$. $\times 16$ in. (orientation 3 ) face were considered. In each orientation, the pulse-height distribution (counts/s) was determined for a given monoenergetic unit flux (photons $/ \mathrm{cm}^{2} / \mathrm{s}$ ). A short utility code was written to take an energy-dependent flux and apply one of the three response functions to determine the total pulseheight distribution. An example of an energy-dependent flux tally is shown in Figure 3.2.5, and its corresponding pulse-height tally was computed using the detector response function for the $2 \mathrm{in} . \times 16$ in. face (orientation 2) and is shown in Figure 3.2.6. 


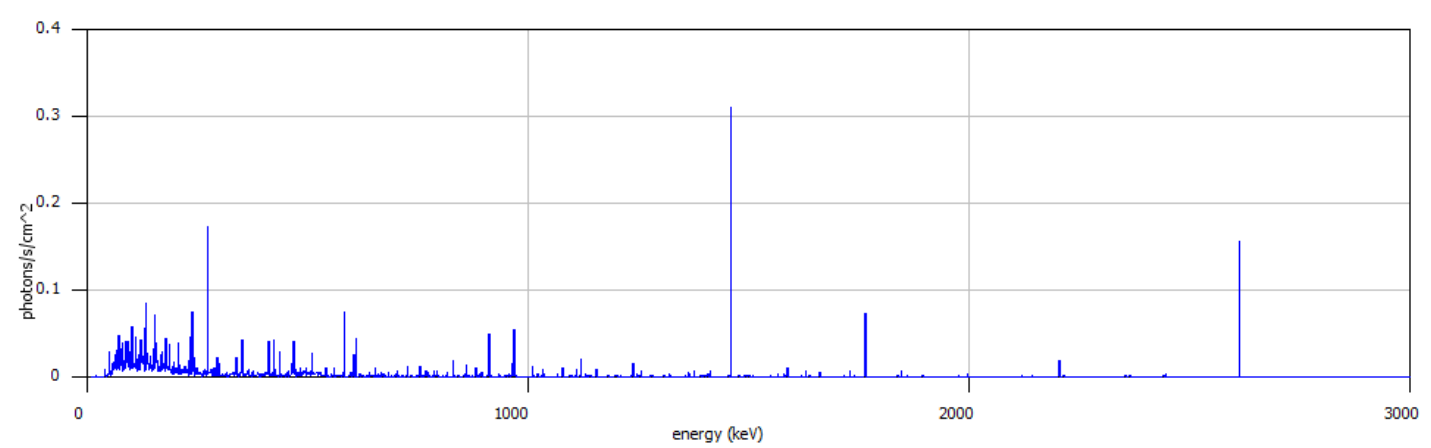

Figure 3.2.5. An example energy-dependent flux tally from SCALE/MAVRIC.

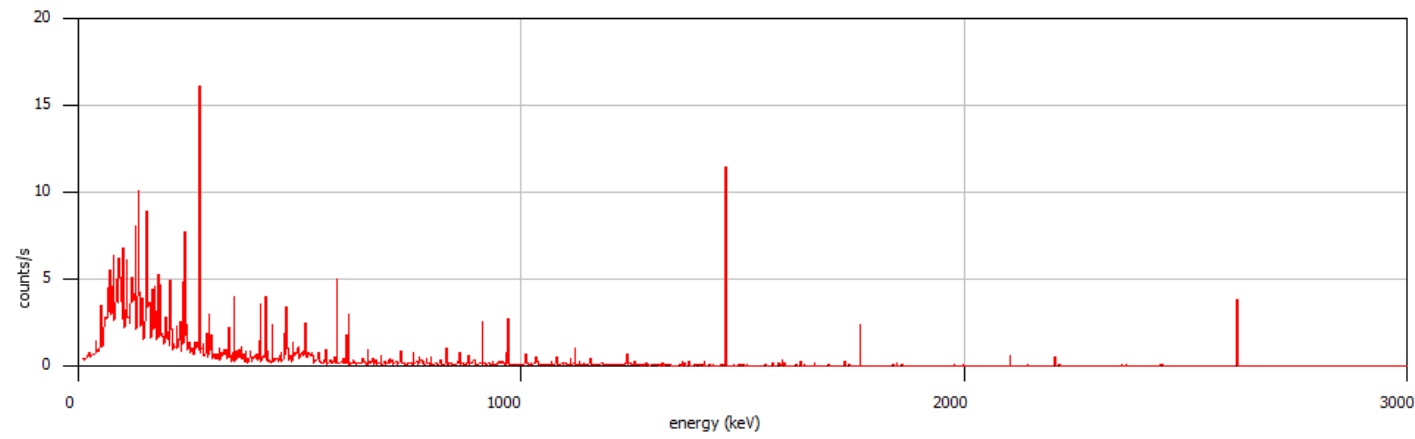

Figure 3.2.6. The pulse-height distribution from the flux computed at the detector location using the 2 in. $\times 16$ in. face response function (orientation 2 ).

Real detectors have an energy-dependent resolution that broadens out the full-energy peaks, which the results provided in Figure 3.2.5 and Figure 3.2.6 do not show. To account for this, another utility program was created to apply an energy resolution function similar to what GADRAS [8] uses. This routine uses the GADRAS equations and parameters for the full width at the half maximum (FWHM) of the distribution as a function of incident energy [8].

$$
\begin{array}{l|ll} 
& E_{\text {crit }}(\mathrm{keV}) & w(\mathrm{keV}) \\
\hline \hline \text { Non-HPGe } & \begin{array}{l}
661 \\
\text { HPGe }
\end{array} & 0.01 P_{7} E_{\text {crit }} \\
\hline \text { FWHM }= & \quad w\left(\frac{E}{E_{\text {crit }}}\right)^{P} & P_{7} \\
& \sqrt{\left[P_{6}\left(\frac{E_{\text {crit }}-E}{E_{\text {crit }}}\right)\right]^{2}+\left[w\left(\frac{E}{E_{\text {crit }}}\right)^{P}\right]_{2}} \quad E \leq E_{\text {crit }} \text { and } P_{6} \geq 0
\end{array}
$$




$$
w\left(\frac{\max \left(E, E_{\text {low }}\right)}{E_{\text {crit }}}\right) P_{8}^{\left[\frac{1}{\ln \left(1-P_{6}\right)}\right]} \quad E \leq E_{\text {crit }} \text { and } P_{6}>0
$$

The parameters $P_{6}, P_{7}$, and $P_{8}$ are supplied by the user along with $E_{\text {low }}=20 \mathrm{keV}$. For the pulse-height distribution shown in Figure 3.2.6, the predicted measured detector spectrum is shown in Figure 3.2.7 (linear plot) and in Figure 3.2.8 (semi-logarithmic plot).

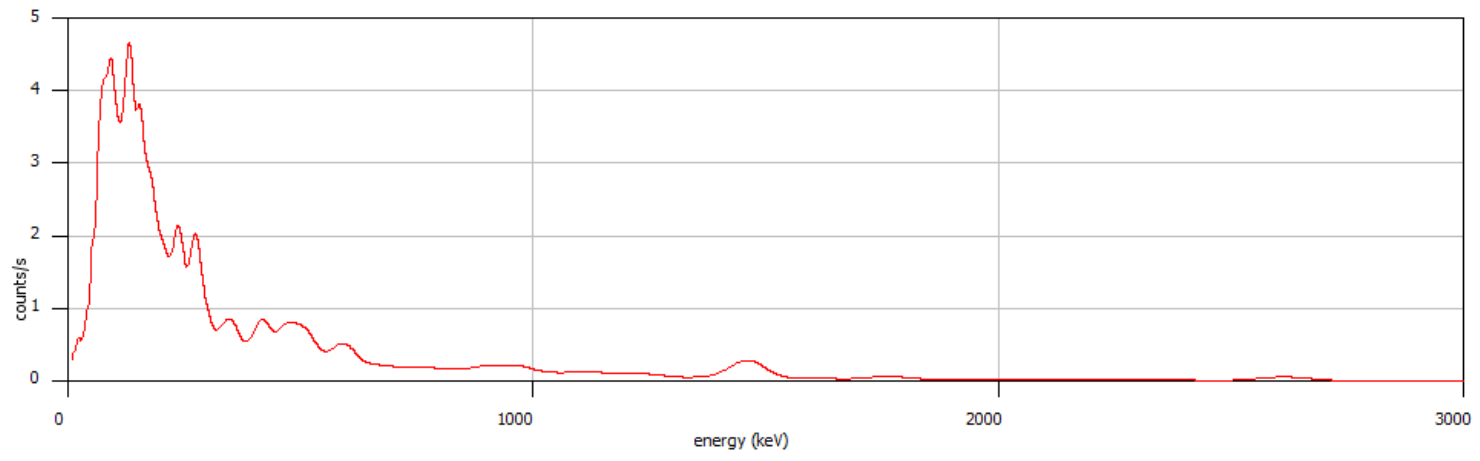

Figure 3.2.7. The predicted energy spectrum (linear scale) for a NaI(TI) (orientation 2). (GADRAS non-HPGe values $\mathrm{P6}=0, \mathrm{P7}=7.5$, and $\mathrm{P8}=0.7$ )

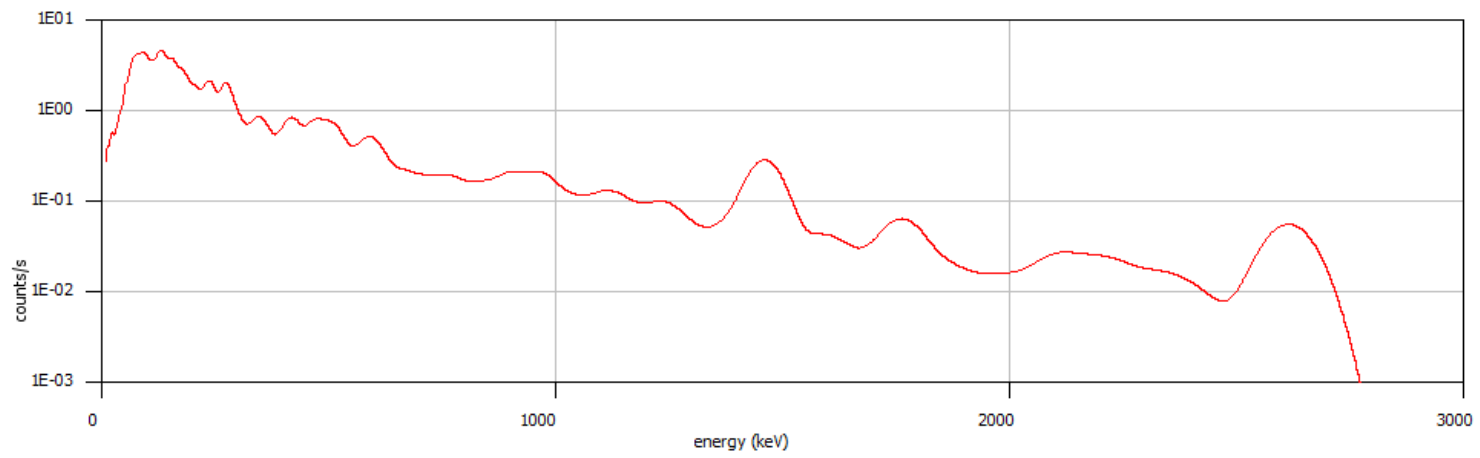

Figure 3.2.8. Predicted energy spectrum (logarithmic scale) for a NaI(TI) (orientation 2). (GADRAS non-HPGe values $\mathrm{P6}=0, \mathrm{P} 7=7.5$, and $\mathrm{P8}=0.7$ )

\subsubsection{Monte Carlo Background Calculations}

For the background simulations, SCALE/MAVRIC was run using 16 separate inputs that required $18 \mathrm{~h}$ each and were then averaged together (poor man's parallel). The energy-dependent flux was tallied at each of the experimental detector locations. After the Monte Carlo, the average flux tally for each detector location was processed with the detector response function for both orientation 2 and orientation 3 of the $\mathrm{NaI}(\mathrm{Tl})$ detector to determine the pulse-height distributions. Energy resolution broadening was then applied to the pulse-height distributions to create the final predicted detector spectra. The simulated 
responses for the seven detector locations were compared to the corresponding experimental measurement values. The detector location at $\mathrm{x}=30 \mathrm{~m}$ is shown in Figure 3.2.9 (linear scale) and Figure 3.2.10 (logarithmic scale). The other detector locations appeared very similar to the results for $\mathrm{x}=30$.

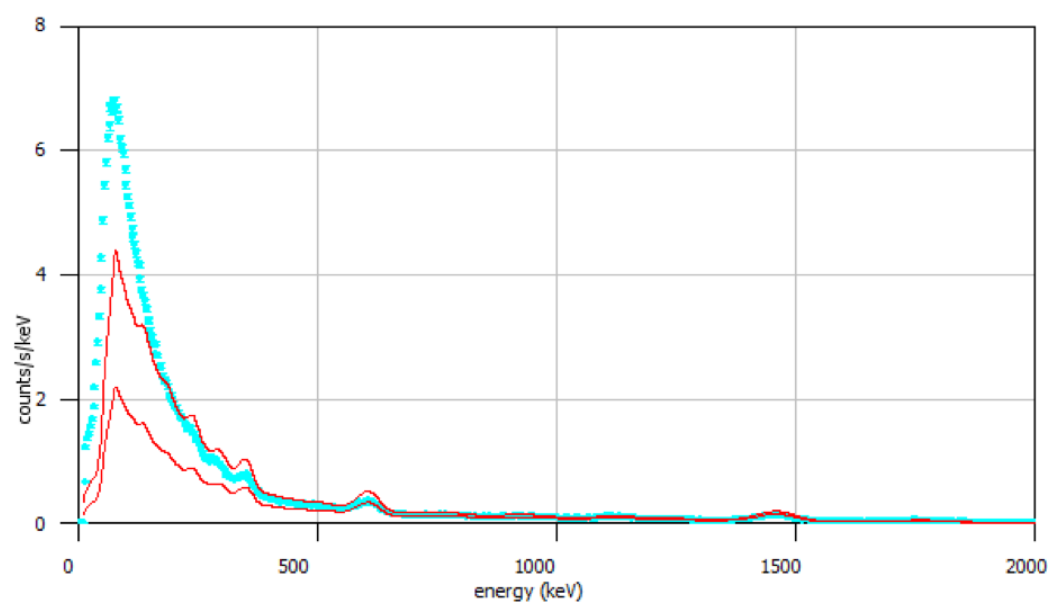

Figure 3.2.9. Simulated and measured background spectra (linear scale) at $x=30 \mathrm{~m}$. Measurements (cyan) include the $1 \sigma$ uncertainties and the simulated results (red) show two detector responses (the $2 \mathrm{in} . \times 16 \mathrm{in}$. face is the lower red line and the 4 in. $\times 16$ in. face is the upper red line).

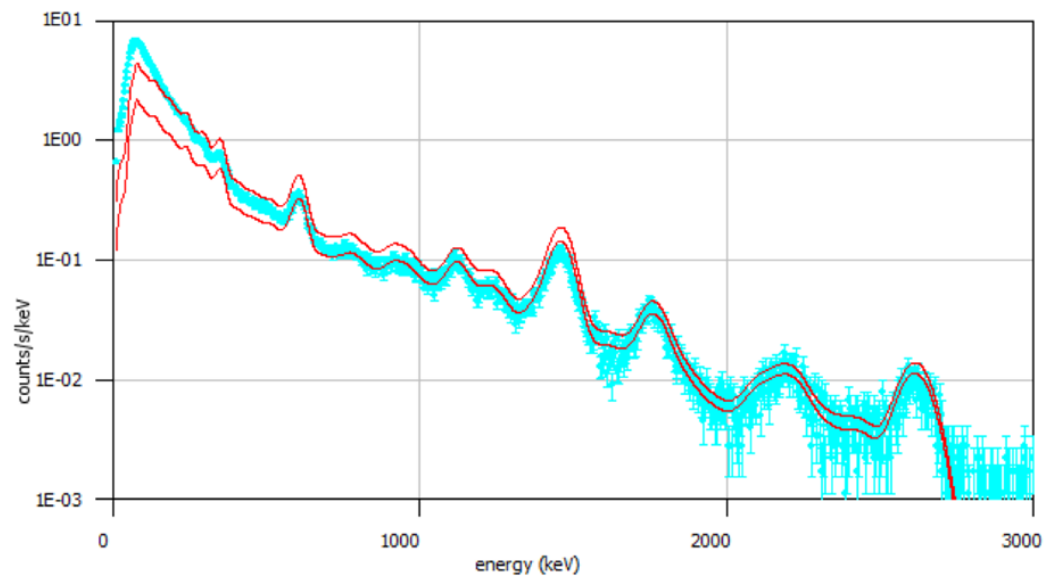

Figure 3.2.10. Simulated and measured background spectra (logarithmic scale) at $x=30 \mathrm{~m}$. Measurements (cyan) include the $1-\sigma$ uncertainties and the simulated results (red) show two detector responses (the 2 in. $\times 16$ in. face is the lower red line and the $14 \mathrm{in.} \times 16 \mathrm{in}$. face is the upper red line).

\subsubsection{Monte Carlo Threat Source Calculations}

For each of the four source locations, SCALE/MAVRIC was run using two separate inputs that required $20 \mathrm{~h}$ each and were subsequently averaged together. The only source included in the simulations was the threat source $(81 \mu \mathrm{Ci}$ of cesium). As described above, the NORM background source was simulated 
separately and can be added to the threat source results to produce the total predicted spectrum from both the threat source and the background.

Equivalent to the background calculations, the energy-dependent flux tallies computed by MAVRIC had two detector response functions (orientation 2 and orientation 3) applied to create pulse-height distributions, and then were broadened to account for detector energy resolution.

Plots showing the comparison of the predicted total detector spectrum (with two detector responses) and the actual measured detector spectrum with the threat source present are shown in Figure 3.2.11 and Figure 3.2.12 for the first source position. The two detectors positions shown are examples of where the direct line-of-sight is blocked (Figure 3.2.11), and the detector has a clear view of the source (Figure 3.2.12). Each plot shows the MAVRIC prediction of background and the total count with the cesium. In Figure 3.2.11, these two predictions are the same because there is no line-of-sight.
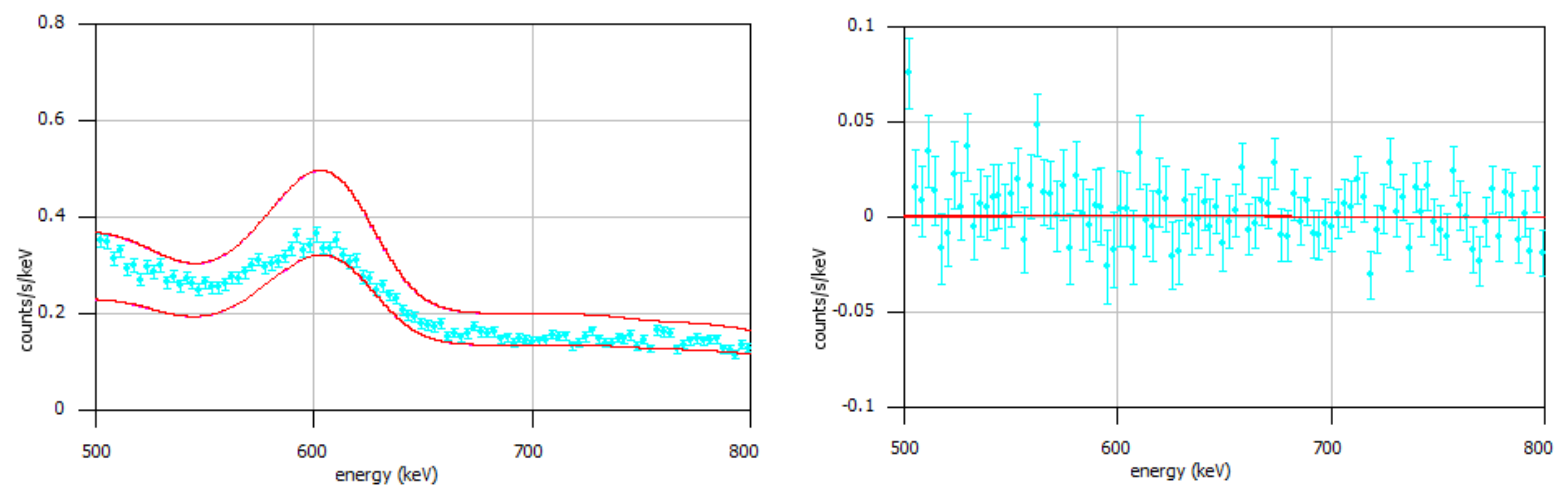

Figure 3.2.11. Comparison of measured (cyan) and simulated (red) detector responses for WAE1 at $10 \mathrm{~m}$. (Left) the full spectrum with cesium source present and simulated background (magenta). (Right) Background-subtracted spectra and the two simulated responses.
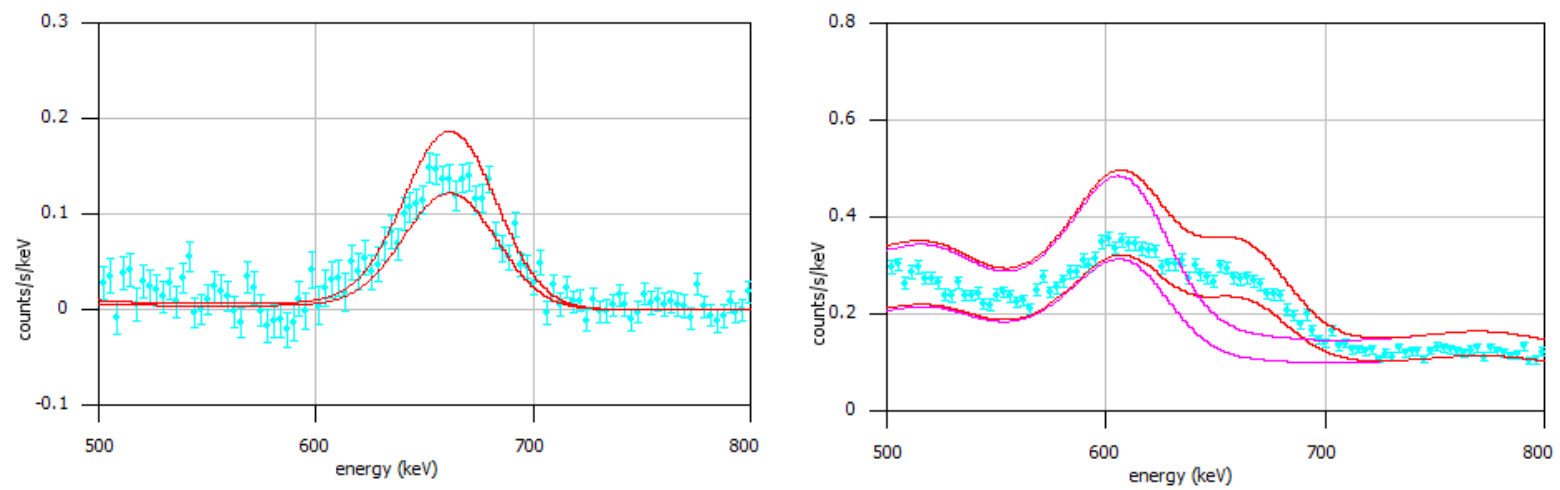

Figure 3.2.12. Comparison of measured (cyan) and simulated (red) detector responses for WAE1 at 20 m. (Left) The full spectrum with cesium source present and simulated background (magenta). (Right) The background-subtracted spectra and the two simulated responses.

\subsubsection{Summary and Next Steps}

MAVRIC was able to compute detailed energy-dependent flux tallies for both the NORM background sources and the threat sources. Assuming the average of the two predicted detector responses matches the actual measured detector spectrum, all of the simulations of NORM background compare very well to measurements in the $1.5-3 \mathrm{MeV}$ range, overpredict the potassium peak at $1.46 \mathrm{MeV}$, slightly overpredict 
between $500 \mathrm{keV}$ and $1.4 \mathrm{MeV}$, and underpredict below $500 \mathrm{keV}$ (by a factor of 2 at $80 \mathrm{keV}$ ). Sensitivity studies need to be done to determine which factors could make such impacts. Possible factors include:

1. the extent of the model that only included Main St. and features near Main St., leaving out the large, square kilometer-sized area around Main St., and a large extent of air may need to be included for skyshine, which impacts the low-energy portion of the spectrum;

2. the presence of any equipment near the detector, which would cause peak energies to be overpredicted and the lower-than-peak scattered component to be underpredicted;

3. if the assumptions regarding material compositions and densities are incorrect, which could change scattering to a small degree; and

4. the assumption that the HPGe measurements were perfectly shielded may have assigned too much source to the material being examined, which could cause the NORM concentration values to be overpredicted.

Some computational studies should be able to determine how these items impact the overall background prediction. Further simulation and analysis is required.

The predictions of the cesium peaks over background matched quite well for source position 1,2 , and 4 . For source position 3, the simulations overpredict the number of cesium peak counts. For the down scattered portion of the spectrum, which would depend on the model geometry and materials more than the peak, the uncertainties in the measured values after background subtraction are too large to make a judgment on a comparison. An example is shown in Figure 3.2.13. Using coarser bins for the background-subtracted measured data, as in Figure 3.2.14, the simulations may be overpredicting the fullenergy peak and underpredicting the low-energy portion of the spectrum. Item 2 from the above list could cause this behavior.

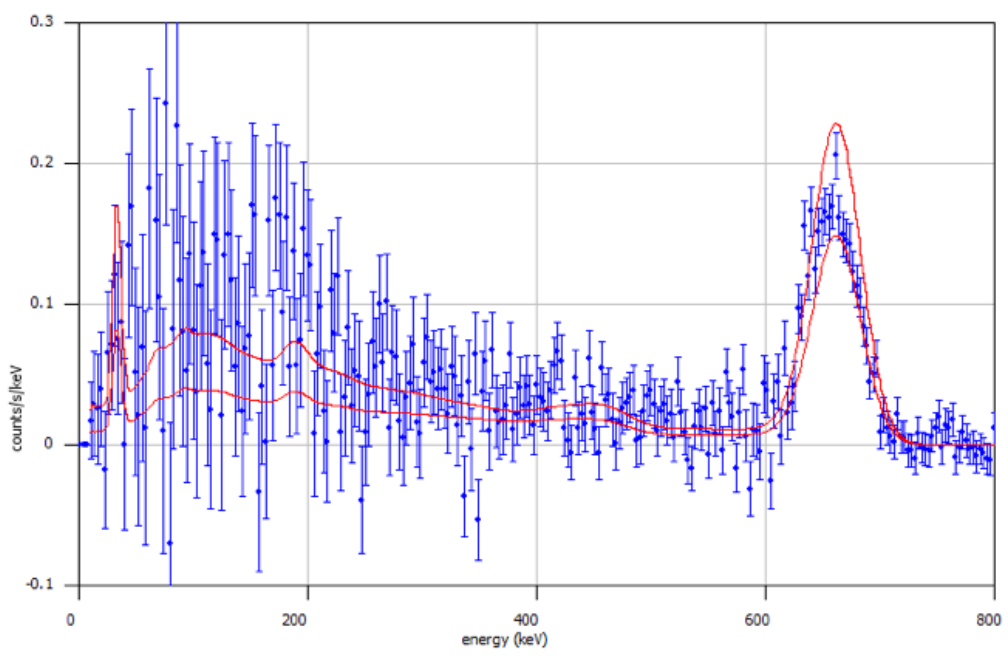

Figure 3.2.13. Comparison of simulated (red) and measured values of background subtracted (blue) counts for source position 1 and detector location $x=30 \mathrm{~m}$. 


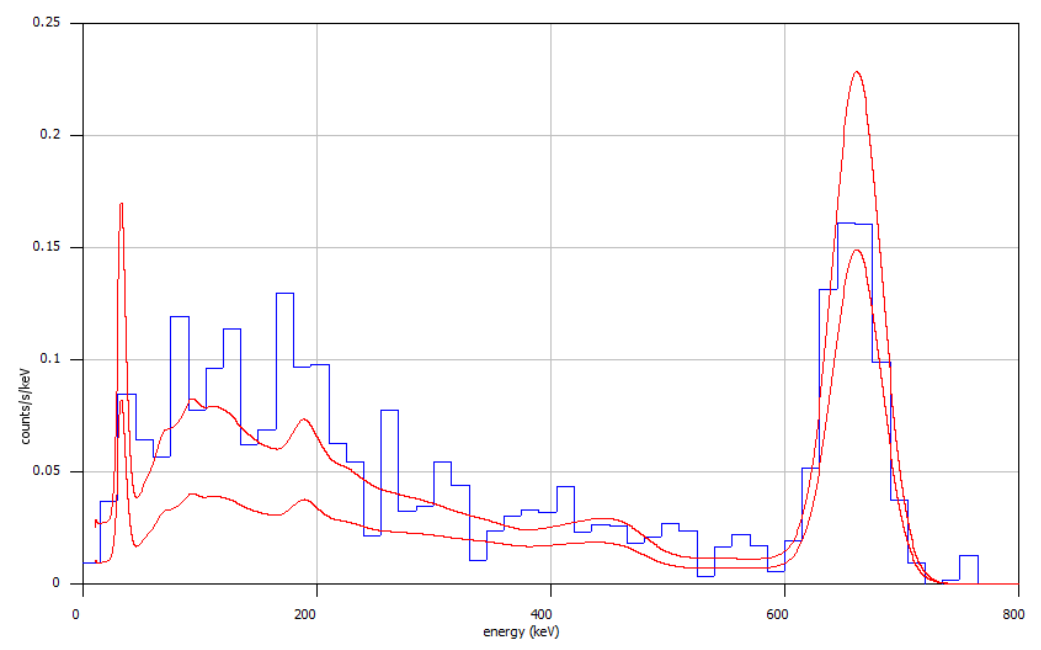

Figure 3.2.14. Comparison of simulated (red) and measured values of background subtracted (blue) counts for source position 1 and detector location $x=30 \mathrm{~m}$. This is the same data as Figure 3.2.13 but with larger bins for measured data. 



\section{MUSE 1 MEASUREMENT CAMPAIGN}

The final data collection at FTIG (MUSE 1) was performed in September 2016. This data collection aimed to measure a variety of radiation sources including special nuclear material (SNM). Because of logistical issues, no radiation sources arrived on-site; however, a smaller set of measurements were still performed because travel to the site had already taken place. These measurements included additional HPGe measurements to address materials lacking measurements and anomalies in previous data. Static $\mathrm{NaI}(\mathrm{Tl})$ background measurements were performed with the cart setups as before as well as controlled dynamic measurements with unmanned ground vehicles using lessons learned from OPTUS 1, 2 and 3. Additional LIDAR data was taken with a different LIDAR unit for comparison to the previous ground truth measurements. An unmanned aerial vehicle (UAV) was also equipped with various optical cameras used to collect stills, video, and hyperspectral data to be used for geometry reconstruction. RadMAP was used to perform static and dynamic measurements across the range site. In addition to the OPTUS/MUSE measurements, additional measurements were performed with multiple backpack platforms being developed through other agencies (Domestic Nuclear Detection Office [DNDO]/ Wearable Intelligent Nuclear Detection [WIND], Defense Threat Reduction Agency [DTRA]/Enhanced Mapping System [EMAPS], and AIPT/NA84).

\subsection{HPGe OUTDOOR BUILDING AND GROUND SURFACE MEASUREMENTS}

During MUSE 1, 16 more HPGe measurements were performed to bridge the remaining gaps perceived to exist by the ORNL research team. Specifically, the goal was to obtain more measurements of the concrete intersections, gravel lots, and more easily interpretable measurements of the cinderblock walls found throughout the FTIG site. A list of the HPGe measurements conducted as a part of the MUSE campaign is shown in Table 4.1.1. The three soil measurements were taken at the very periphery of the site to determine how much the NORM concentration differs from soil found within the interior of the site. This was of particular concern because modeling the effects of skyshine depends on the sources around the site. All of the MUSE measurements were used in the final NORM determination analysis, bringing the total number of HPGe NORM measurements to 70. Separated by material, this includes asphalt (13), soil (12), gravel (7), intersections (5), sidewalks (9), foundations/patios (11), and cinder block walls (13).

Table 4.1.1. Comprehensive List of MUSE HPGe Measurements.

\begin{tabular}{|c|c|c|c|}
\hline ID & Material & Description & File Name \\
\hline H71 & Soil & Soil behind townhouse, down hill & Runix08-H71_Soil-2016-09-01T12.24.38.631 \\
\hline $\mathrm{H} 72$ & Block, light tan & $\begin{array}{l}\text { 1-story residence building } 07 \mathrm{~b} \text {, outer east } \\
\text { wall, facing outwards }\end{array}$ & $\begin{array}{l}\text { Runix08-H72_Wall_Bldg_07B-2016-09- } \\
\text { 01T14.04.06.834 }\end{array}$ \\
\hline $\mathrm{H} 73$ & $\begin{array}{l}\text { Concrete, } \\
\text { intersection }\end{array}$ & Intersection of Main and Church, center & $\begin{array}{l}\text { Runix08-H73_Concrete-2016-09- } \\
01 T 15.19 .32 .518\end{array}$ \\
\hline H74 & Block, dark tan & $\begin{array}{l}\text { 2-story townhouse building } 10 \text {, outer } \\
\text { south wall, facing outwards }\end{array}$ & $\begin{array}{l}\text { Runix08-H74_Wall_Bldg10-2016-09- } \\
\text { 01T17.59.28.262 }\end{array}$ \\
\hline H75 & Soil & Soil behind police station & Runix08-H75_Soil-2016-09-02T08.51.48.347 \\
\hline H76 & Block, red & $\begin{array}{l}\text { Police station rubble building } 05 \text {, outer } \\
\text { west wall, facing outwards }\end{array}$ & $\begin{array}{l}\text { Runix08-H76_Wall_Bldg05-2016-09- } \\
\text { 02T09.59.40.907 }\end{array}$ \\
\hline H77 & $\begin{array}{l}\text { Concrete, } \\
\text { intersection }\end{array}$ & Intersection of Main and Maple, center & $\begin{array}{l}\text { Runix08-H77_Concrete-2016-09- } \\
\text { 02T11.17.07.915 }\end{array}$ \\
\hline $\mathrm{H} 78$ & $\begin{array}{l}\text { Concrete, } \\
\text { intersection }\end{array}$ & Intersection of Maple and Road 4, center & $\begin{array}{l}\text { Runix08-H78_Concrete-2016-09- } \\
\text { 02T12.20.19.472 }\end{array}$ \\
\hline H79 & Soil & Soil east of site, beyond road 4 & Runix08-H79_Soil-2016-09-02T13.15.35.637 \\
\hline
\end{tabular}


Table 4.1.1. Comprehensive List of MUSE HPGe Measurements. (continued)

\begin{tabular}{|c|c|c|c|}
\hline ID & Material & Description & File Name \\
\hline $\mathrm{H} 80$ & Block, dark tan & $\begin{array}{l}\text { Half-wall outdoors near road } 4 \text {, outer } \\
\text { east wall, facing outwards }\end{array}$ & Runix08-H80_Wall-2016-09-02T14.20.30.704 \\
\hline $\mathrm{H} 81$ & Block, light tan & $\begin{array}{l}\text { 1-story store building } 01 \mathrm{a} \text {, outer east } \\
\text { wall, facing outwards }\end{array}$ & $\begin{array}{l}\text { Runix08-H81_Wall_Bldg_01A-2016-09- } \\
\text { 02T15.19.59.529 }\end{array}$ \\
\hline $\mathrm{H} 82$ & Gravel & $\begin{array}{l}\text { Between 2-story office bldg. } 02 \text { and 1- } \\
\text { story shop bldg. 01B }\end{array}$ & $\begin{array}{l}\text { Runix08-H82_Gravel-2016-09- } \\
02 \mathrm{~T} 16.29 .21 .565\end{array}$ \\
\hline $\mathrm{H} 83$ & Gravel & Service station, near pumps & $\begin{array}{l}\text { Runix08-H83_Gravel-2016-09- } \\
02 \mathrm{~T} 17.22 .30 .736\end{array}$ \\
\hline $\mathrm{H} 84$ & Asphalt & Main St., in front of bar & $\begin{array}{l}\text { Runix08-H84_Asphalt-2016-09- } \\
\text { 03T08.06.11.170 }\end{array}$ \\
\hline H85 & Gravel & $\begin{array}{l}\text { Between OPS/storage bldg. } 12 \text { and hotel } \\
\text { bldg. } 08 \text {, center }\end{array}$ & $\begin{array}{l}\text { Runix08-H85_Gravel-2016-09- } \\
\text { 03T08.58.36.186 }\end{array}$ \\
\hline H86 & Asphalt & Main St., in front of hotel & $\begin{array}{l}\text { Runix08-H86_Asphalt-2016-09- } \\
\text { 03T09.50.39.012 }\end{array}$ \\
\hline
\end{tabular}

After the campaign, the combined HPGe measurements from all the OPTUS and MUSE campaigns was reanalyzed, and count rates from the most prominent gamma-ray peaks were determined. An example spectrum highlighting some of these peaks is illustrated in Figure 4.1.1. These count rates were used in conjunction with detector responses predicted from numerous MCNP6 simulations to make determinations about the concentration of NORM in all the various materials found throughout the FTIG site. The following discussion briefly describes the methodology followed to reach these final NORM predictions along with the final results and is reproduced from Refs. [9] and [10].

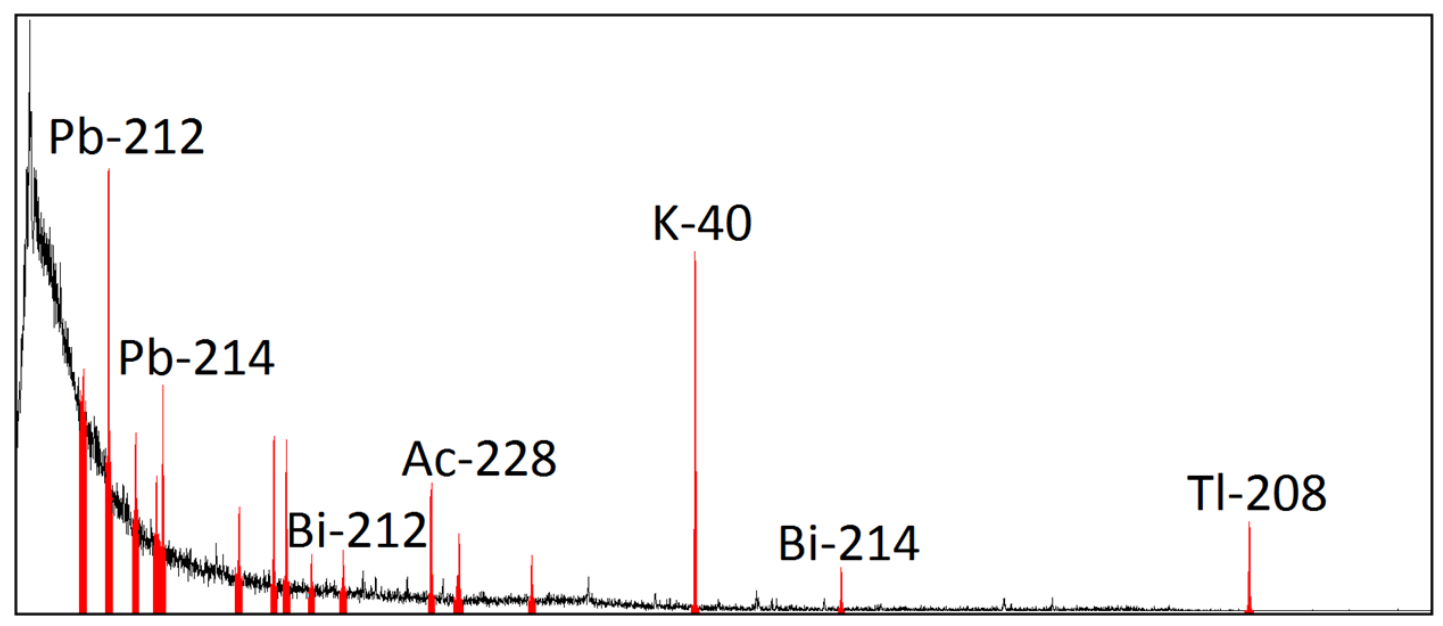

Figure 4.1.1. An example spectrum obtained from a soil measurement with regions containing peaks of interest highlighted in red.

To calculate the concentration of NORM in a given material, the following equation was implemented, which expresses the full-energy peak count rate in a detector as:

$$
C(E)=K * g * R(E)
$$


where:
$C(E)$ is Count rate of peak at energy $\mathrm{E}(\mathrm{cps})$,
$K$ is the activity concentration $(\mathrm{Bq} / \mathrm{kg})$,
$g$ is the branching ratio ( $\gamma /$ decay), and
$R(E)$ Is the detector response at energy $\mathrm{E}$ (counts per $\gamma / \mathrm{kg}$ ).

Twenty different peaks (from 186.2 to $2614.5 \mathrm{keV}$ ) were used to estimate the NORM concentrations of the primary parent constituents $\left({ }^{40} \mathrm{~K},{ }^{238} \mathrm{U}\right.$, and $\left.{ }^{232} \mathrm{Th}\right)$ in conjunction with nuclear data and the detector response predicted using MCNP6 simulations. Secular equilibrium of the parents and daughters was assumed, and variance weighted averages were implemented when multiple peaks were used to estimate daughter and parent activities. The detailed MCNP6 models that were used to estimate the detector response from a distributed photon source were critical in order to translate the measured gamma spectra into estimates of activity concentrations (of potassium, uranium, and thorium) in soil, concrete, asphalt, etc. A comparison of the detector to the HPGe model is shown in Figure 4.1.2.

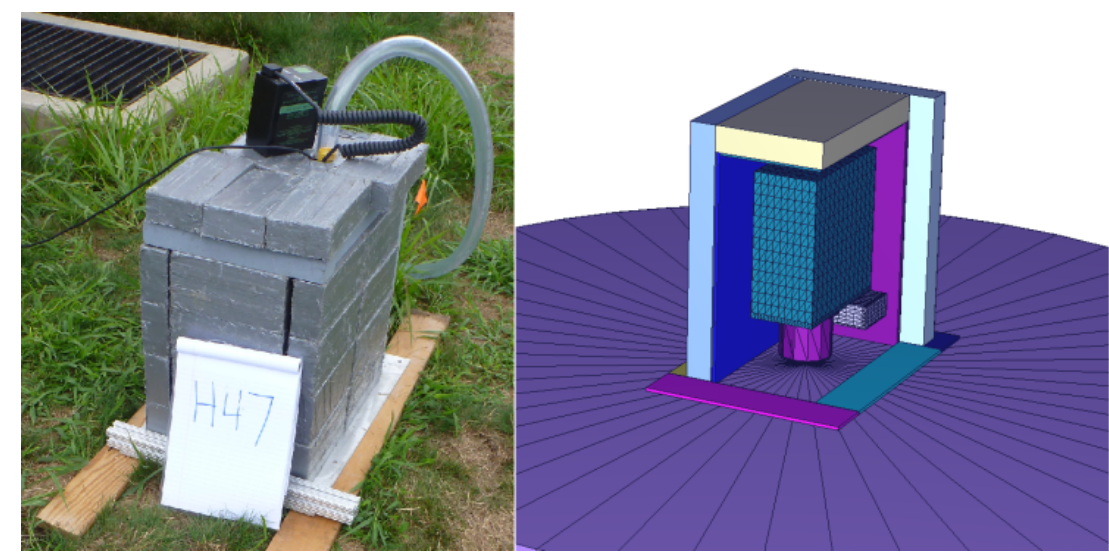

Figure 4.1.2. Comparison of the ORTEC EX-100T detector surrounded by lead for measurement H47 (left) to the MCNP6 model developed for this effort (right).

Preliminary simulations included the replication of controlled measurements of standard sources in a lab setting (to establish confidence in the model) and soil geometries with various depths to ensure that enough ground was simulated. Once confidence in the model was established and the response was determined for simple single-layer geometries, NORM concentrations were calculated for soil, concrete, and asphalt. Using the predicted NORM concentrations as a source in the same MCNP6 models, synthetic data was generated to ensure internal consistency.

Once the single-layer NORM concentrations were determined, these values were used as a source in twolayer geometries (e.g., gravel and sidewalk), and for the more complicated wall geometries. By estimating the contribution coming from below the gravel or sidewalk or beyond a wall, this contribution could be subtracted from the measurement, and the remaining signal could be attributed to the material being examined. Agreement of the activity predicted from various peaks originating from the same nuclide was used to ensure this method was working correctly. For example, there was better agreement of the activity predicted from the 583.2 and $2,614.5 \mathrm{keV}^{208} \mathrm{Tl}$ peaks in the sidewalk when the asphalt pavement source was used beneath the thin concrete layer as compared to when soil was assumed to be under the sidewalk. Because these two photons are attenuated very differently by the sidewalk above, their relative agreement 
can be used as a check. When soil was used in this case (containing more thorium), too many counts were subtracted from the $2,614.5 \mathrm{keV}$ peak, which resulted in a large disagreement in the predicted ${ }^{232} \mathrm{Th}$ concentration in the sidewalk.

Figure 4.1.3 illustrates one of the measurements that was modeled using this method with an example of a wall at the FTIG site composed of concrete masonry units (CMUs) where the ground on the other side of the detector consisted of soil, a sidewalk, and an asphalt roadway. [5]

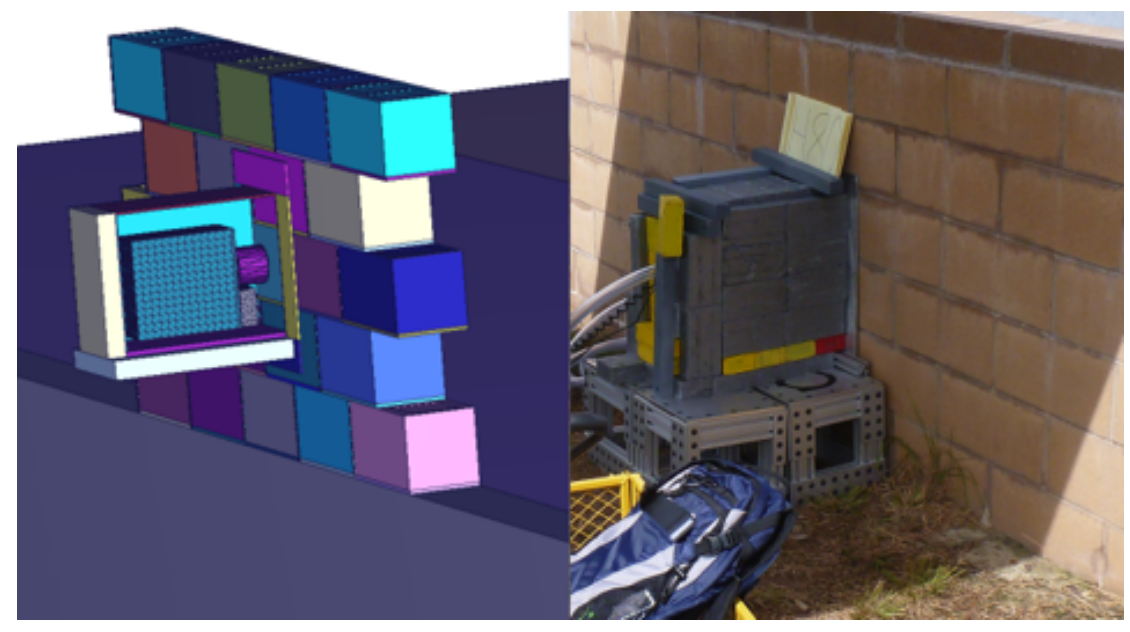

Figure 4.1.3. Example of an MCNP6 simulation designed to predict the contribution from background sources beyond a CMU wall (left) and the actual experimental setup (right).

Figure 4.1.4 illustrates the full distributed source used to predict the contribution to the spectrum measured by the HPGe detector from the soil, sidewalk, and asphalt roadway beyond the CMU wall.

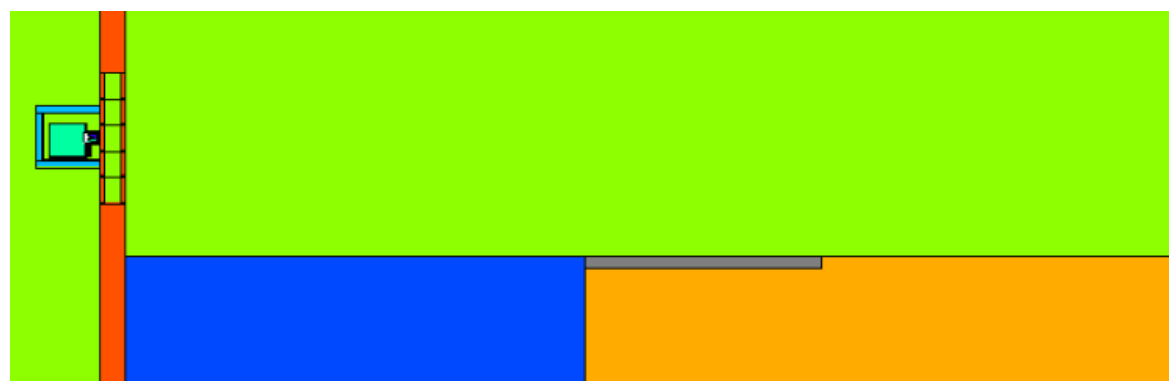

Figure 4.1.4. A side view of the MCNP6 simulation geometry used to estimate the detector response through a CMU wall (red, left) from a distributed source in soil (blue), sidewalk (grey), and asphalt pavement (orange).

These photon sources were generated using ORIGEN to predict the full gamma source from $1 \mathrm{~Bq}$ of each parent nuclide with all its daughters in secular equilibrium. This photon spectrum (with $1 \mathrm{keV}$ bins) was then scaled appropriately using the predicted NORM concentrations for each material and inserted in the MCNP6 input as a gamma source. The ADVANTG [10] code was then implemented to bias the source and generate appropriate weight windows to improve the statistics of the final MCNP6 detector response calculation.

Once the spectra from the various measurements were analyzed, the response function for each geometry was characterized, and any contributions from sources beneath or beyond the material were estimated, the activities of all relevant nuclides could be calculated. The results from all 70 measurements are displayed in Table 4.1.2, as an average for each material type. The uncertainties given in Table 4.1.2 represent the 
sample standard deviation for the collection of measurements for a given material type. This uncertainty illustrates the variability of the NORM concentration across the site, as represented by a finite number of measurements (given in parentheses). Because there was only one available measurement of a red CMU wall, the uncertainties listed for these values are estimates of the uncertainty of the NORM concentration arising from uncertainty in peak areas, nuclear data, and the predicted response function. In general, the typical propagation of errors for the other values would result in smaller uncertainties than those displayed - some of which would be misleading because of the variability of the NORM values across the site.

Table 4.1.2. Calculated NORM activity concentrations for various materials at FTIG (Bq/kg).

\begin{tabular}{l|cccc}
\hline $\begin{array}{l}\text { Material (No. of } \\
\text { Measurements) }\end{array}$ & ${ }^{40} \mathbf{K}$ & ${ }^{238} \mathbf{U}$ & ${ }^{232} \mathbf{T h}$ & ${ }^{137} \mathbf{C s}^{*}$ \\
\hline \hline Soil (12) & $420 \pm 55$ & $26.5 \pm 2.4$ & $37.7 \pm 3.4$ & $5.4 \pm 2.1$ \\
Intersections (5) & $227 \pm 8$ & $15.5 \pm 1.5$ & $9.6 \pm 0.6$ & - \\
Sidewalks (9) & $230 \pm 15$ & $16.2 \pm 1.2$ & $10.9 \pm 0.4$ & - \\
Foundations (11) & $200 \pm 26$ & $16.3 \pm 1.4$ & $9.8 \pm 0.6$ & - \\
Asphalt (13) & $91 \pm 6$ & $23.7 \pm 0.8$ & $3.7 \pm 0.5$ & - \\
Gravel (7) & $139 \pm 34$ & $20.0 \pm 2.9$ & $4.6 \pm 1.5$ & - \\
Light Tan CMU (4) & $239 \pm 25$ & $13.4 \pm 0.9$ & $9.3 \pm 1.6$ & - \\
Dark Tan CMU (4) & $122 \pm 55$ & $9.2 \pm 2.0$ & $8.0 \pm 3.0$ & - \\
White CMU (2) & $72 \pm 5$ & $8.2 \pm 0.3$ & $4.7 \pm 0.3$ & - \\
Grey CMU (2) & $165 \pm 8$ & $12.8 \pm 0.4$ & $5.7 \pm 0.5$ & - \\
Red CMU (1)** & $103 \pm 9$ & $10.2 \pm 0.6$ & $5.7 \pm 0.5$ & - \\
\hline * Detectable amounts of ${ }^{137}$ Cs were only found in soil. \\
** Uncertainty represents a single measurement, not uncertainty in value across the \\
FTIG site.
\end{tabular}

Figure 4.1.5 displays the data in the form of a box plot, which shows all of the measurements split into quartiles, with the box representing the center $50 \%$ and the outer ticks demarking the smallest and largest measurements. It is apparent that the three concrete measurements (intersections, foundations, and sidewalks) show similar values and trends. The asphalt pavement and gravel NORM values also show some similarities, although there is significant spread to the gravel values. This is likely from a variety of factors, including a large amount of variability in the depth of the gravel and material beneath the gravel lots. The wall values also have a lot of spread partially because there were intrinsic differences in NORM concentrations as a function of type (Table 4.1.2). 


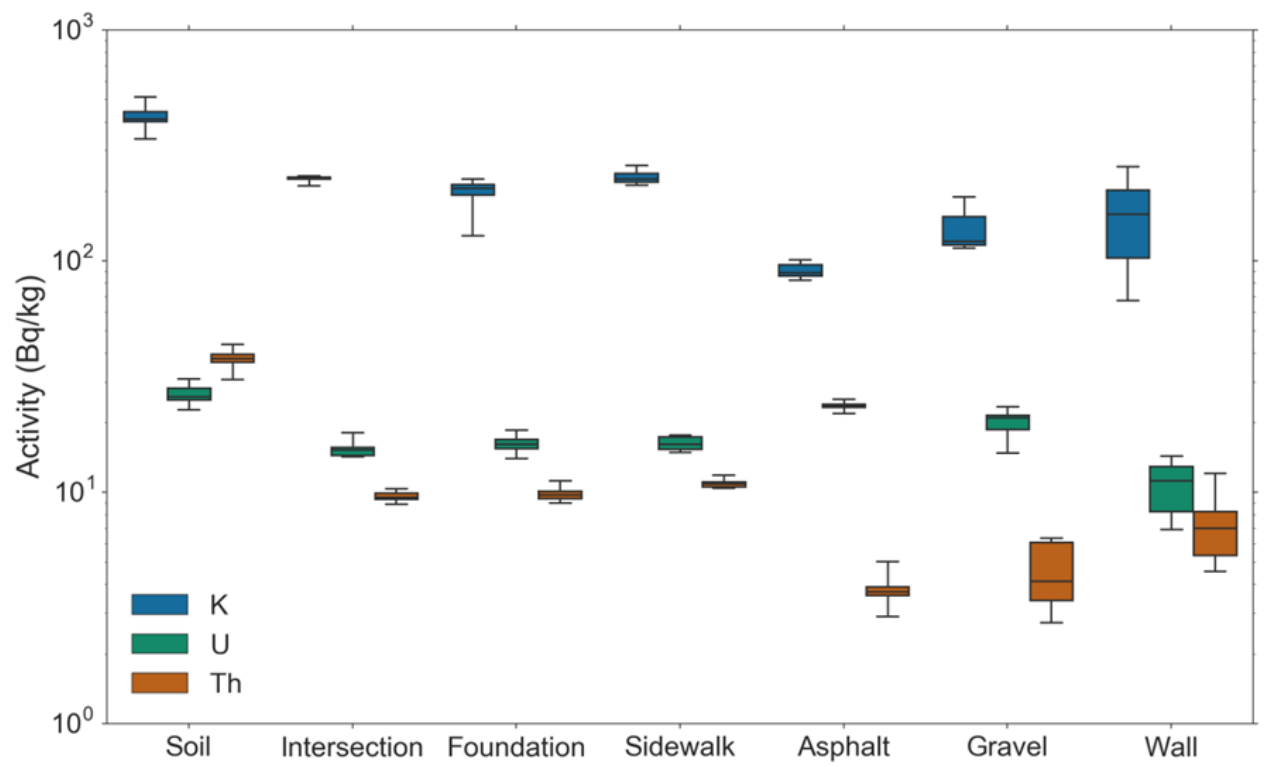

Figure 4.1.5. NORM concentrations of 70 material measurements across FTIG. Note that the ticks are not error bars but represent the bounds of the determined NORM values for each material based on individual measurements.

Aside from agreement with worldwide accepted NORM values and internal consistency, synthetic data was generated from the determined concentrations to establish confidence in the final NORM values. In general, the synthetic data generated from the MCNP6 simulations matched to within less than 10\% for most of the full-energy peaks and to within $\sim 12 \%$ of the entire spectra from 200 to $3,000 \mathrm{keV}$. The synthetic data consistently underpredicted the spectra below $200 \mathrm{keV}$, which was expected because none of the environment behind the lead cave was modeled. Radiation attenuated through or leaking around the lead bricks from buildings, the ground, and skyshine would tend to add to this low-energy continuum. This discrepancy could also be partially attributed to cosmic ray interaction with the lead shielding itself, resulting in a low-energy cascade.

\subsection{NaI(TI) STATIC BACKGROUND MEASUREMENTS}

Only background measurements were taken during the MUSE 1 measurement campaign because no industrial, medical, or SNM sources were available. Ninety-two static measurements, $1 \mathrm{~m}$ apart, were taken down Main St. during MUSE 1 with gain stabilization and a highly refined data acquisition system using lessons learned from OPTUS 1 and OPTUS 2. The energy scaled total count rates, as a function of position can be seen in the upper left portion of Figure 4.2.1 for MUSE 1 (filled blue circles) and OPTUS 1 (empty black circles). The count rates in MUSE 1 are lower than those measured in OPTUS 1. The reason for this discrepancy is not known but may be attributed to the lack of gain stabilization and calibration information in the OPTUS 1 campaign. NORM peak areas, shown in the upper right portion of Figure 4.2.1, are similar for both OPTUS 1 and MUSE 1.

The MUSE 1 static measurements show an anomaly between nearest neighbor measurements in that fluctuations, both increase and decrease, are present between measurements. During the campaign, measurements were originally taken $2 \mathrm{~m}$ apart in the morning of Day 2 and then the gaps were filled in the afternoon to produce $1 \mathrm{~m}$ increments. During this time, there was a systematic variation in the total count rate, which is confirmed by a nearby radiation detector connected to a weather station. See Section 4.6 for more information. 
Before the MUSE 1 measurement campaign, the Monte Carlo detector response calculations were performed, using NORM concentrations measured in OPTUS 1 and 2 and validated by $\mathrm{NaI}(\mathrm{Tl})$ measurements taken in OPTUS 3. A comparison of these calculations and the MUSE 1 measurements are shown in the lower left portion of Figure 4.2.1. The overall magnitude of the $\mathrm{NaI}(\mathrm{Tl})$ measurements and Monte Carlo simulations are different, but the change in count rate as a function of position is similar. For positions greater than $80 \mathrm{~m}$, Monte Carlo and static measurements diverge. This is because of edge effects of the Monte Carlo model, which ends just after the $90 \mathrm{~m}$ position. Further refinements to the Monte Carlo models are underway, including adding in skyshine and improving detector response calculations. These static measurements provide a high-quality benchmark for future Monte Carlo detector response calculations.
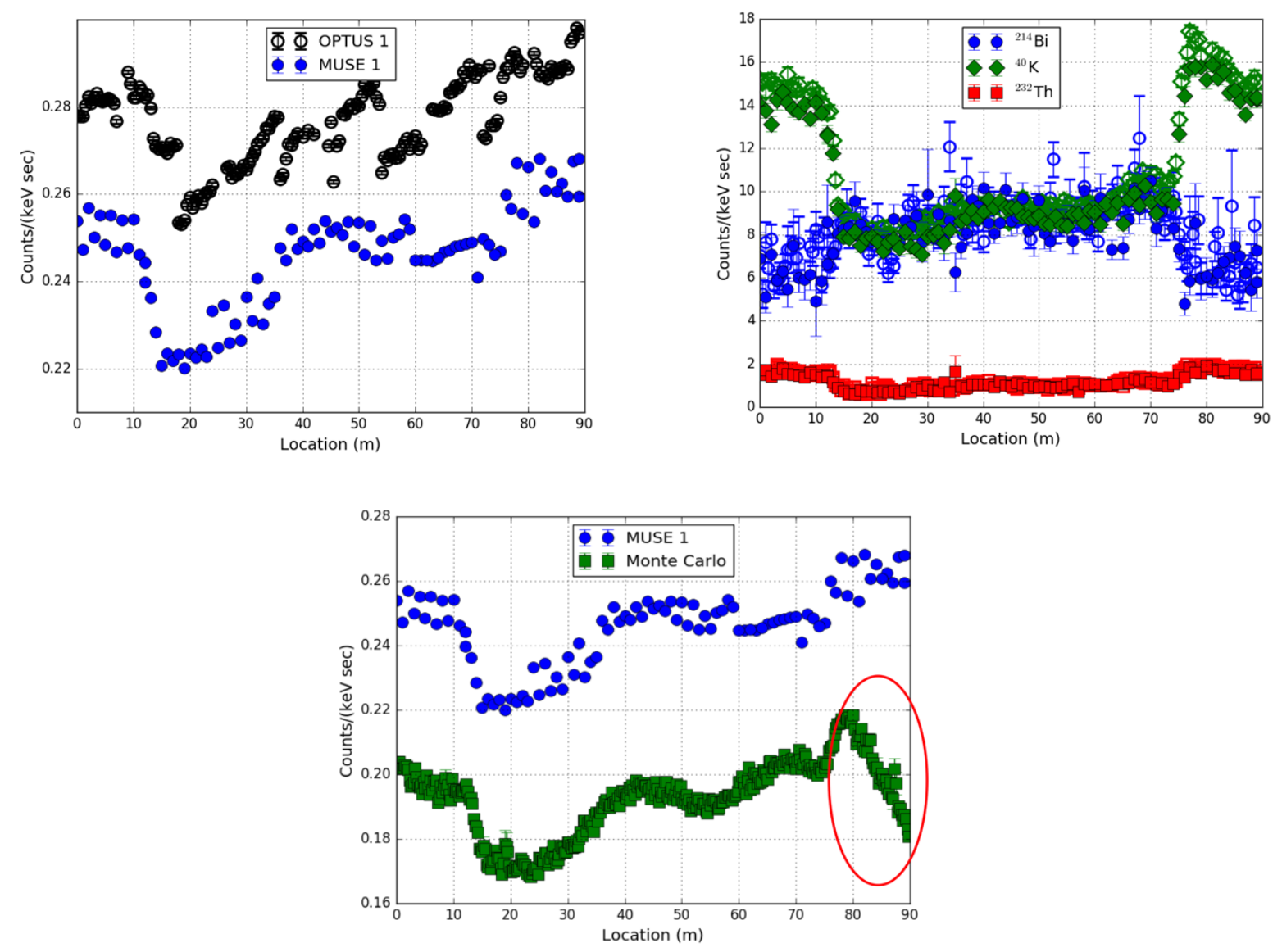

Figure 4.2.1. OPTUS 1 and MUSE 1 measurement comparisons. (Top left) MUSE 1 static measurements compared with OPTUS 1 measurements. (Top right) ${ }^{214} \mathrm{Bi},{ }^{40} \mathrm{~K}$, and ${ }^{232} \mathrm{Th}$ peak areas for OPTUS 1 (empty markers) and MUSE 1 (filled markers). (Bottom) MUSE 1 static measurements and Monte Carlo particle transport detector response function calculations. Although the predicted Monte Carlo response functions closely track the variance in the static measurements, the magnitude is less.

\subsection{NaI(TI) DYNAMIC BACKGROUND MEASUREMENTS}

In addition to static measurements, dynamic measurements down Main St. were taken using a robot pulling the $\mathrm{NaI}(\mathrm{Tl})$ cart moving at 2.8 and $1.4 \mathrm{mph}$. See the left portion of Figure 4.3.1 for an example 
dynamic measurement path. This data is used to validate the sampling and interpolation methodology described in Section 0. The right of Figure 4.3.1 shows the MUSE 1 static, dynamic and synthetic total count rates for the $2.8 \mathrm{mph}$ measurements.
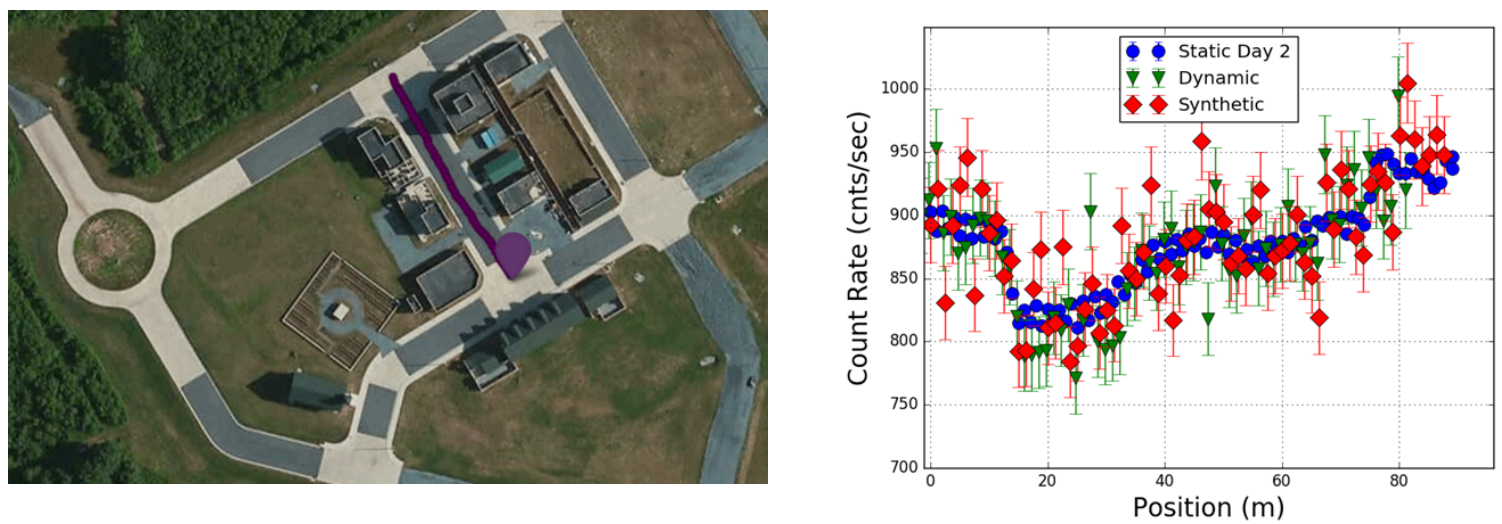

Figure 4.3.1. MUSE 1 static, dynamic, and synthetic data comparison. (Left) Path of moving 2 in. $\times 4$ in. $\times$ 16 in. NaI(Tl) detector down Main St. at $1.23 \mathrm{~m} / \mathrm{s}$. (Right) Total count rate of static measurements, dynamic measurement and synthetic data generated from the static data.

To understand the effect of systematic variations in the background as a function of position on generated synthetic data, many synthetic datasets were created using varying amounts of static measurements. The right side of Figure 4.3.2 show the total count rates for static, dynamic and synthetic datasets for synthetic datasets based on every other static measurement, every 24,48 and 64 static measurements. These correspond to 2,24, 48, and $64 \mathrm{~m}$ distances between static measurements used to generate synthetic data. It should be noted that the first and last static measurements were always used, meaning that the difference between the next to last and last static measurement location used may be less than other locations. This choice was made to provide interpolated detector response calculations across the entire measurement path. 

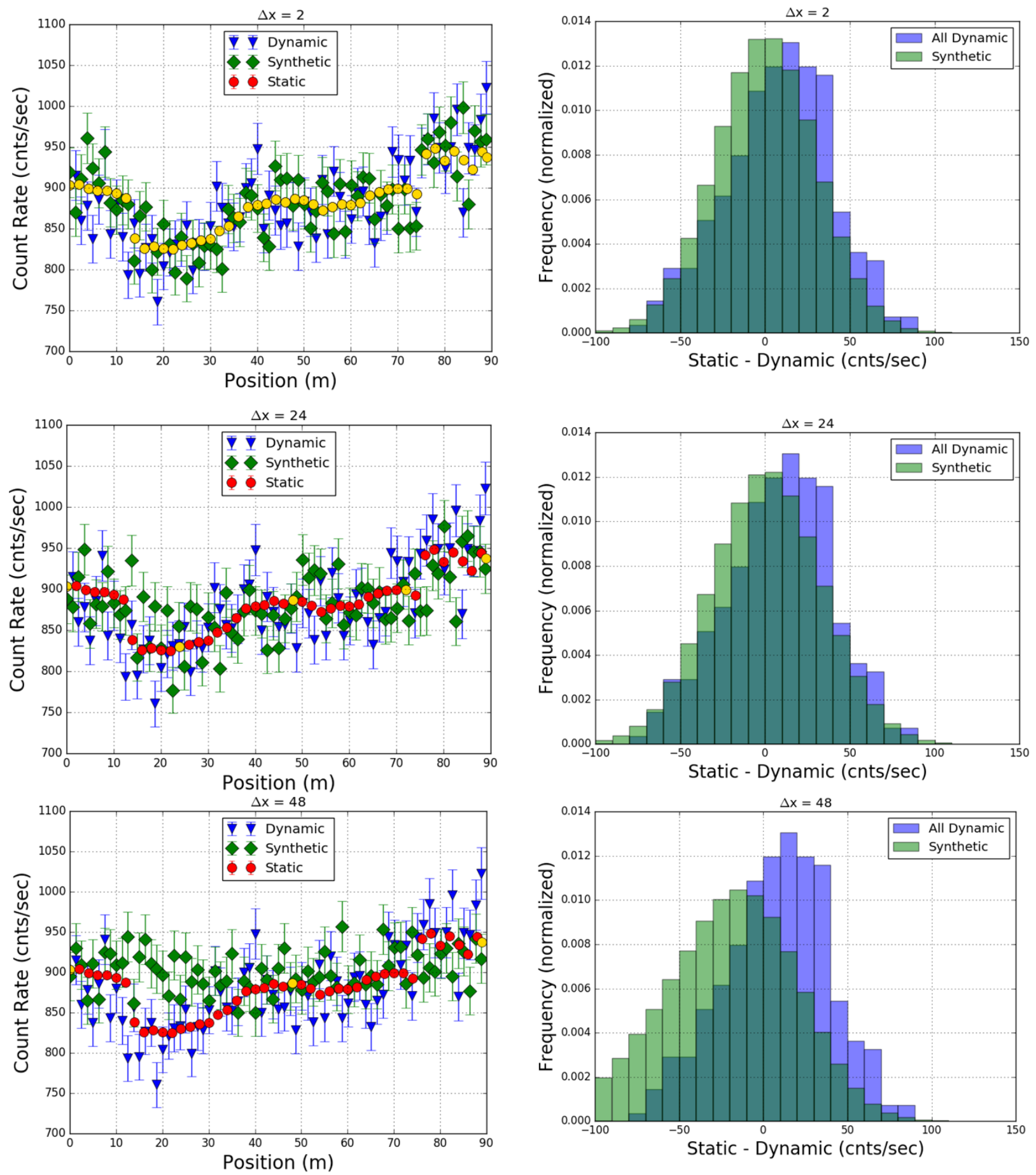

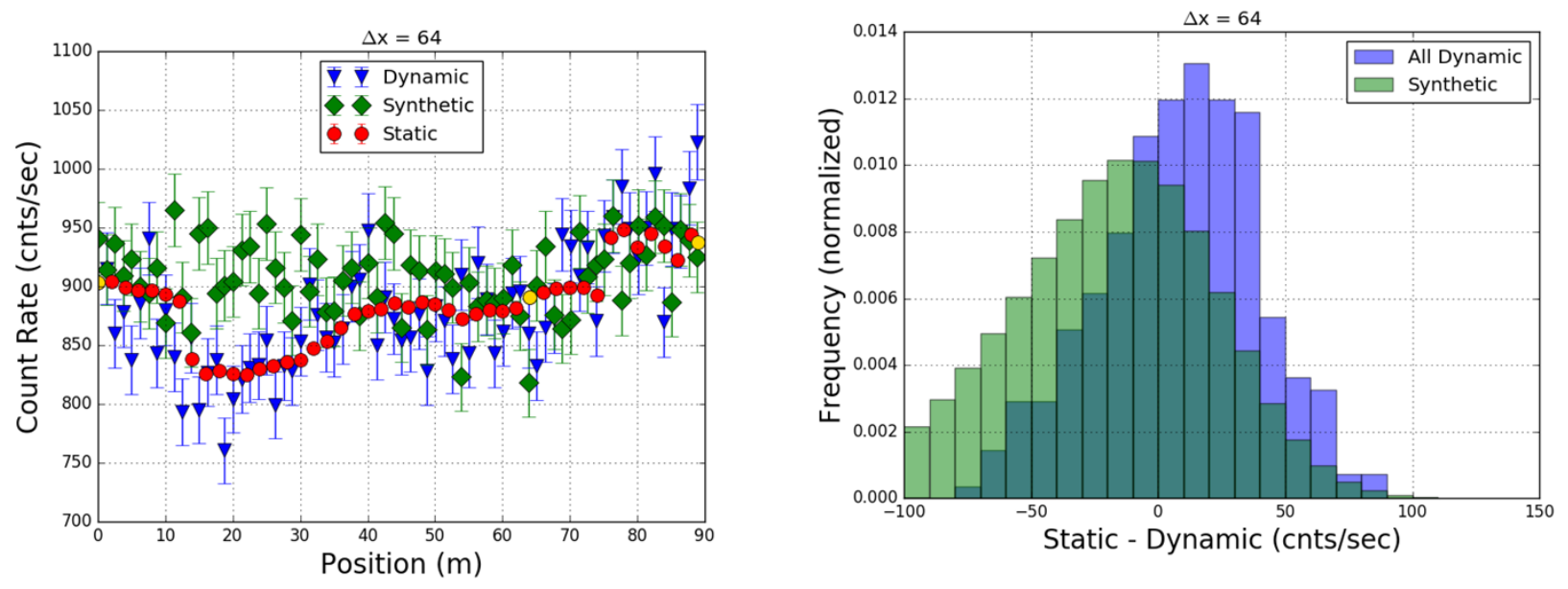

Figure 4.3.2. MUSE 1 measured static and dynamic datasets compared to calculated synthetic data. (Left) Comparison of static, dynamic and synthetic datasets. Gold markers within the red dataset denote static measurements used for synthetic dataset generation. (Right) Normalized histogram of difference between dynamic and synthetic datasets using varying amounts of static measurements in synthetic data generation.

The right column of Figure 4.3.2 shows the histogram difference between dynamic and static measurements and synthetic and static measurements. The width of each distribution arises from statistical variations in short measurements, but the means are very similar around 0 , or no difference. The dynamic means are systematically larger. This difference may be attributed to a small change in measurement geometry between static and dynamic measurements. The mean (marker) and standard deviation (error bar) for all difference histograms simulated synthetic data generated from every other measurement to every 64 measurements is shown in Figure 4.3.3. After the difference between measurements reaches $32 \mathrm{~m}$ (or every 32 measurements), there is an increase in the difference between static and dynamic measurements due to the shape in the measured background as a function of position along Main St.

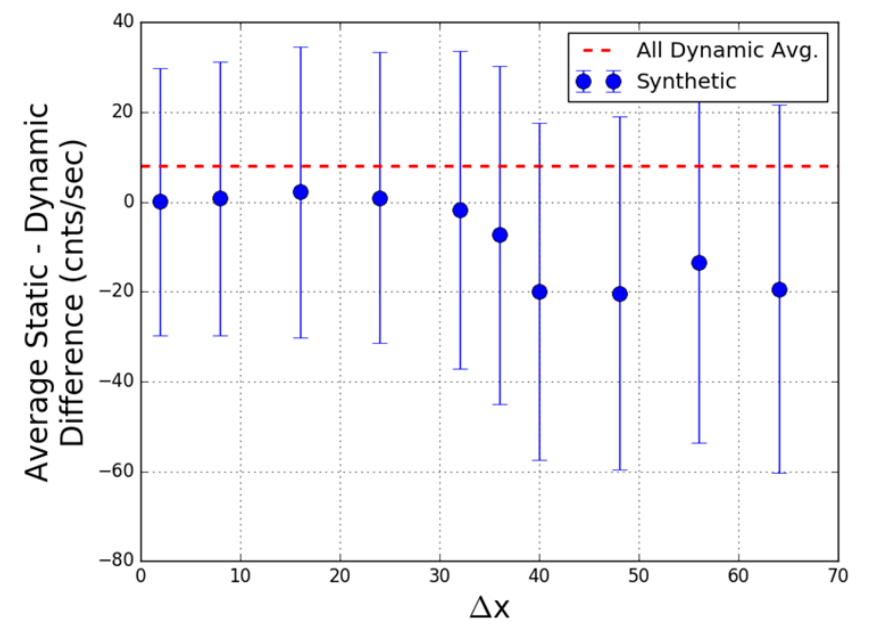

Figure 4.3.3. Average static/dynamic difference with 1- $\sigma$ error. After changing the difference between positions used for interpolation is $>\mathbf{3 0} \mathbf{~ m}$, a shift in the synthetic difference mean is observed. For an environment with larger background variations, this distance will decrease. 


\subsection{GEOREFERENCING}

For the MUSE 1 campaign, static measurements were performed along the centerline of Main St. Because of time constraints and equipment errors, DGPS points were not able to be taken with centimeter accuracy for each measurement. However, the reference point for $(0,0)$ at the intersection of the concrete joint of Main St. and Church St. was used as a known location. Using a measuring tape, the 92 measurement points were marked in increments of $1 \mathrm{~m}$ along the center of Main St. as was done in previous campaigns. In addition to the measurement locations of the static data taken, dynamic data points were also georeferenced. To monitor the location of the robot and cart, they were placed at point $(0,0)$, and the motor encoder data was used to verify a constant speed from the starting point to the ending point along Main St. The HPGe measurement locations were documented with photographs and relative GPS locations as shown in Figure 4.4.1.

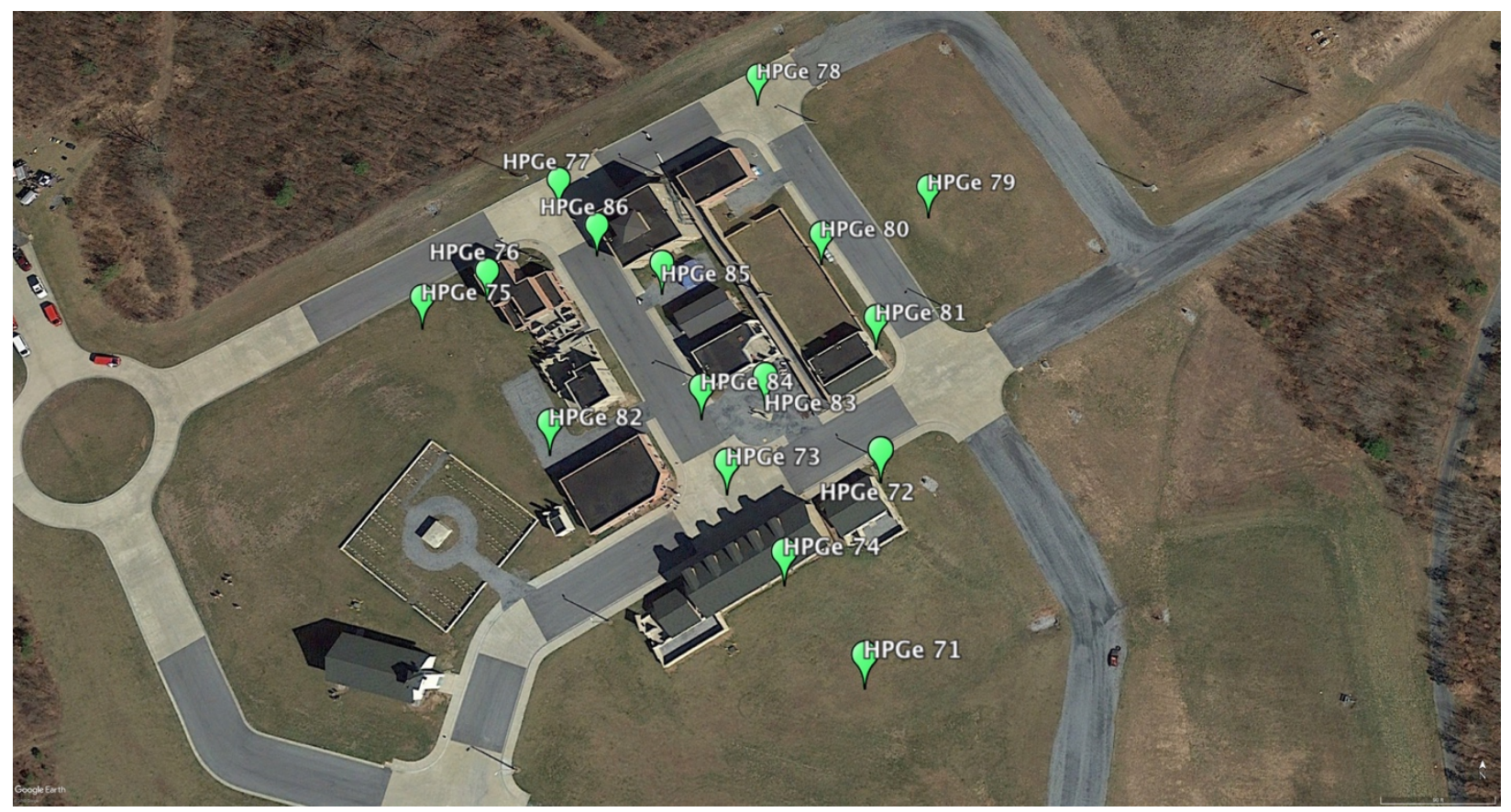

Figure 4.4.1. Locations of MUSE 1 HPGe measurements.

\subsection{LIDAR/VISUAL MAPPING OF CACTF BUILDING EXTERIORS}

LIDAR and imagery data of FTIG were taken by three platforms fielded by ORNL. Analysis of the data - including construction and model generation - are a part of the fiscal year (FY) 2017 scope of tasking. Details of sensory data acquired by the three platforms are discussed below.

\subsubsection{Unmanned Aerial System (UAS)}

A total of 10 flights were conducted around the FTIG area varying flight path, altitude, time of day, motion in the scene, and camera sensor parameters. Sensors included a visible high pixel count point-andshoot camera as well as a low-resolution thermal imager. 


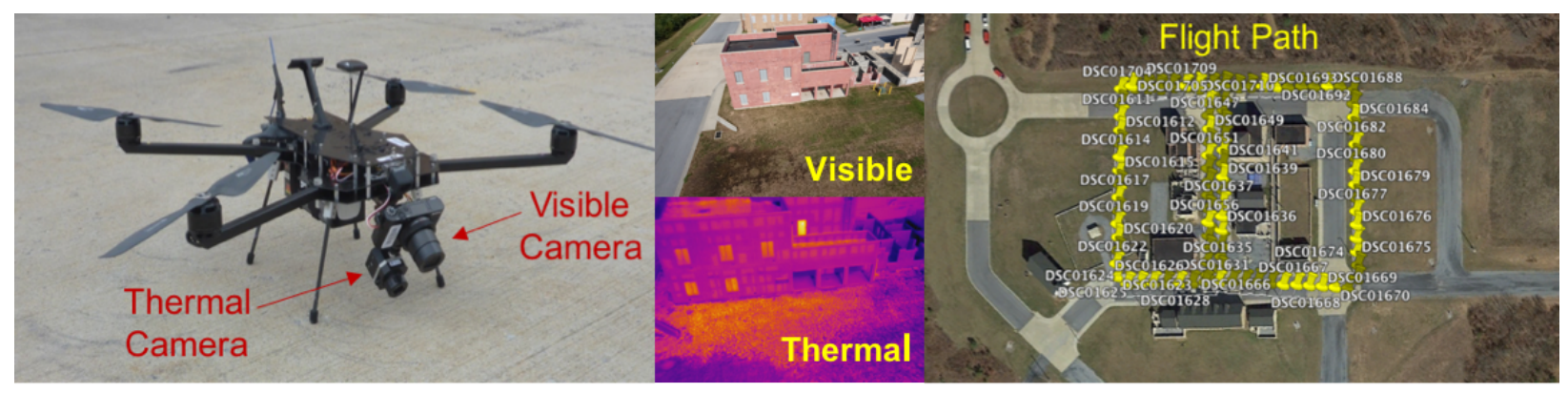

Figure 4.5.1. UAS visible and thermal imaging system. (Left) UAS system and sensors used for data acquisition. (Middle) Example of visual sensor data collected with the UAS. (Right) Example of a flight plan used for a collection. Alternate paths followed the perimeter of FTIG.

Using the visual imagery captured from the UAS, five point clouds with position and coloring were stitched together using structure-from-motion software (Figure 4.5.2). A comparison of the generated point clouds with the ground truth LIDAR from the FY 2015 campaign has been conducted and is shown in Figure 4.5.3. Fidelity of geometry capture for these flights has been found to be within a few inches of ground truth for most of the generated points. Close-in flights show tighter agreement with ground truth than flights at a larger standoff distance.

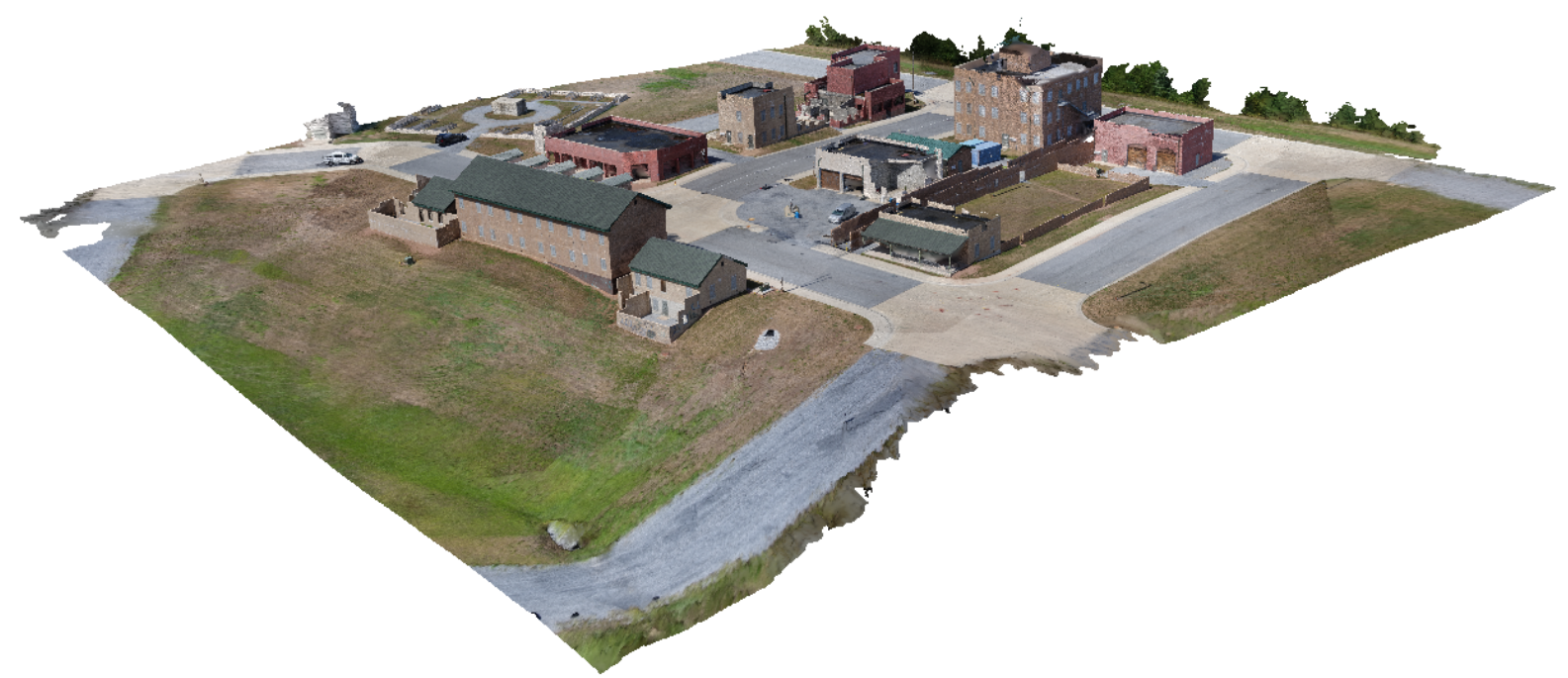

Figure 4.5.2. Point cloud generated from a combination of visible photographs and UAS platform. 

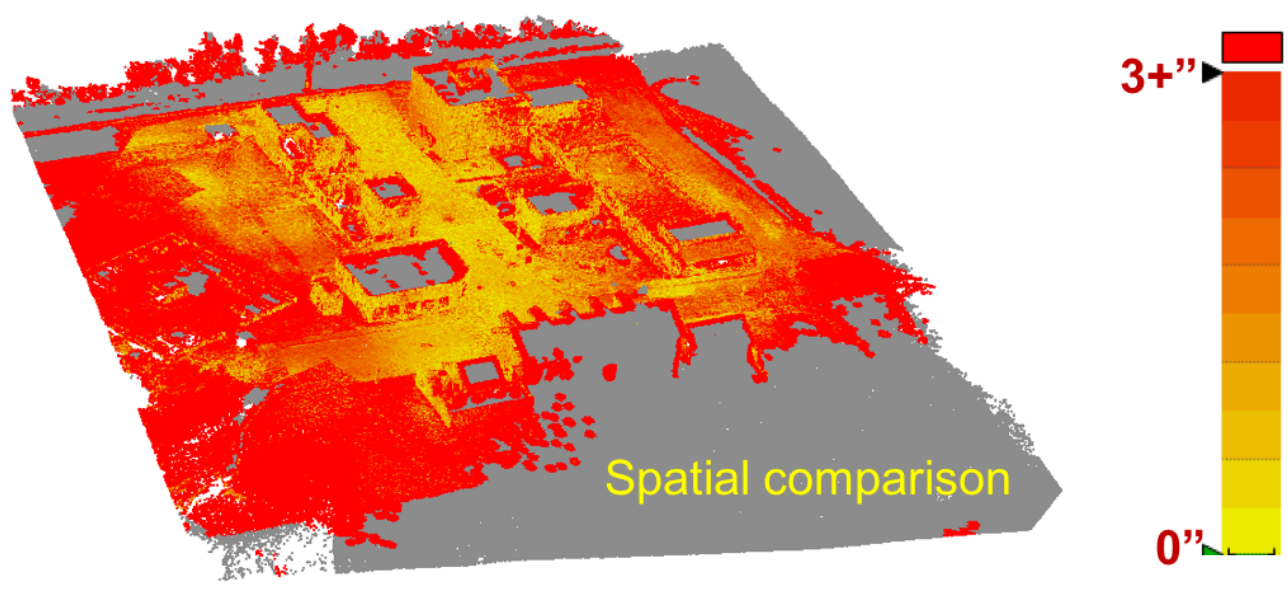

Figure 4.5.3. Comparison of UAS point cloud versus point cloud from long-dwell LIDAR collected during FY 2015 campaigns.

\subsubsection{Terrestrial Robot}

About a dozen runs were made along the streets of FTIG to capture the exterior of buildings with a focus on the Main St. area. Onboard sensors included a pair of wide lens cameras for spherical $360^{\circ}$ images and a single VLP-16 LIDAR unit (Figure 4.5.4). Various combinations of sensors were live during the runs, and auxiliary navigational and odometry data was measured from the motor encoders on the robot.

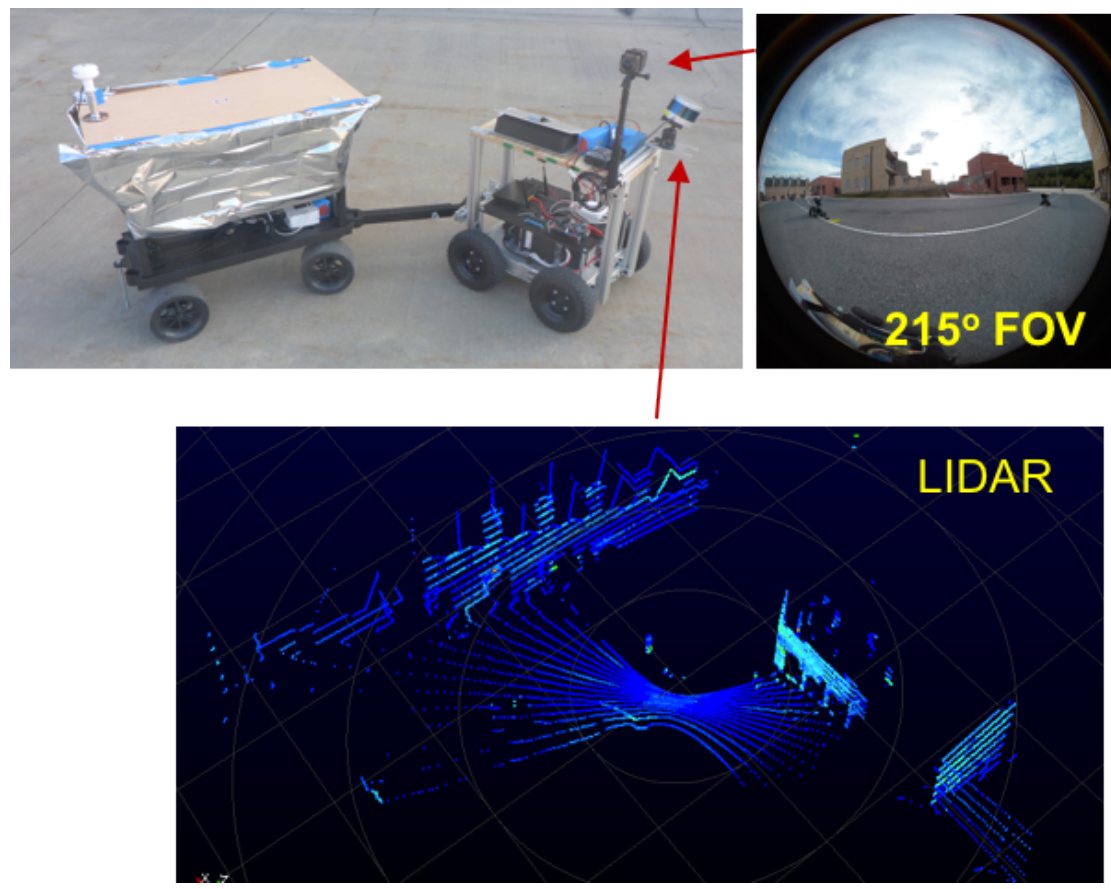

Figure 4.5.4. Terrestrial robot acquisition system with LIDAR and $360^{\circ}$ camera system coupled to radiation detection cart.

Initial point clouds have been constructed using the imagery and the LIDAR as standalone sources.

Imagery processed in the same manner as the UAS flights demonstrated poor registration (i.e., incomplete point clouds), likely caused by interference from the sun and sky. Initial LIDAR stitching was performed 
using the iterative closest point method (without navigational measurement support) and was found to be partially successful over short spans of measurements. Development aimed at improving the registration algorithms for stitching the LIDAR sources using tandem inertial measurement unit (IMU) data is currently underway. This will serve as the geometry backbone of the model. Visible photographs will then be projected onto the LIDAR-derived geometry for color texturing.

\subsubsection{Terrestrial Pedestrian System}

Two passes were performed in the interior of the hotel and a portion of the townhouse using a pedestrian system composed of a mounted LIDAR, point-and-shoot camera, and the $360^{\circ}$ camera on a platform that was carried by a human. This data has yet to be analyzed, but the team anticipates the developments with the terrestrial robot will translate to stitching point clouds on this system. Although no navigational system (e.g., IMU) was present, it will be possible to generate fictitious IMU data using the crude iterative closest point algorithm. This localization data will then feed into a more robust simultaneous localization and mapping algorithm for geometry creation. Stitching a geometry using imagery alone will be attempted, but it is anticipated that the best geometries will likely result from imagery coloring the LIDAR-derived geometry as in the terrestrial robot platform.

\subsection{METEOROLOGICAL AND PERSISTENT RADIATION MONITORING}

The MUSE 1 campaign used two new weather/persistent radiation monitoring (WPRM) stations developed at ORNL with the following features:

- Two separate Davis Vantage Pro weather stations with additional soil moisture sensors to study effects of precipitation;

- Autonomous persistent radiation monitoring detector with a 2 in. $\times 4$ in. $\times 16$ in. $\mathrm{NaI}(\mathrm{Tl})$ crystal(s) paired with ORTEC MCA DigiBase;

- Raw ${ }^{40} \mathrm{~K}$ peak tracking-based active gain stabilization;

- Weather data time synchronized with persistent gamma spectral data and conveniently stored in a .sqlite3 database; and,

- Easily accessible from data acquisition system used for other $\mathrm{NaI}(\mathrm{Tl})$ measurements.

Both weather monitoring stations were equipped with a single Davis weather station and a single autonomous persistent radiation monitoring detector system as shown in Figure 4.6.1. 


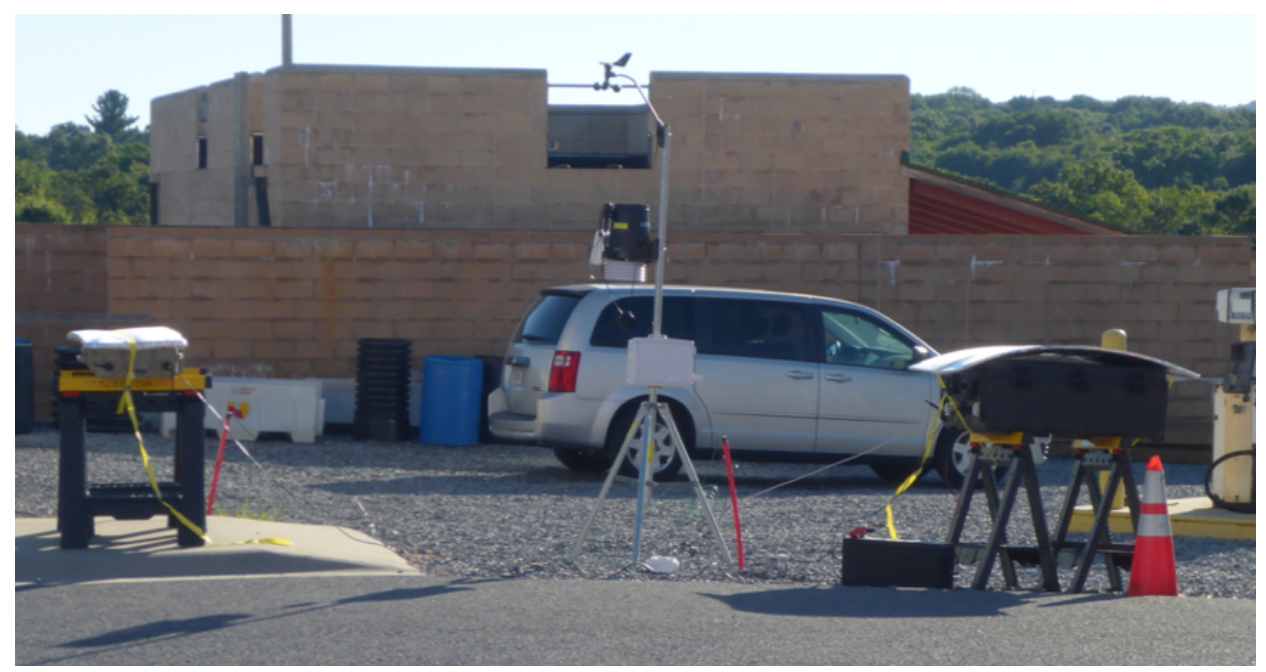

Figure 4.6.1. WPRM-2 with Davis weather station and two NaI(Tl) detectors.

The WPRM-1 was placed near the roundabout at the CACTF, whereas the WPRM-2 station was placed near the garage/gas station at the corner of Main St. and Church St. as shown in Figure 4.6.2.

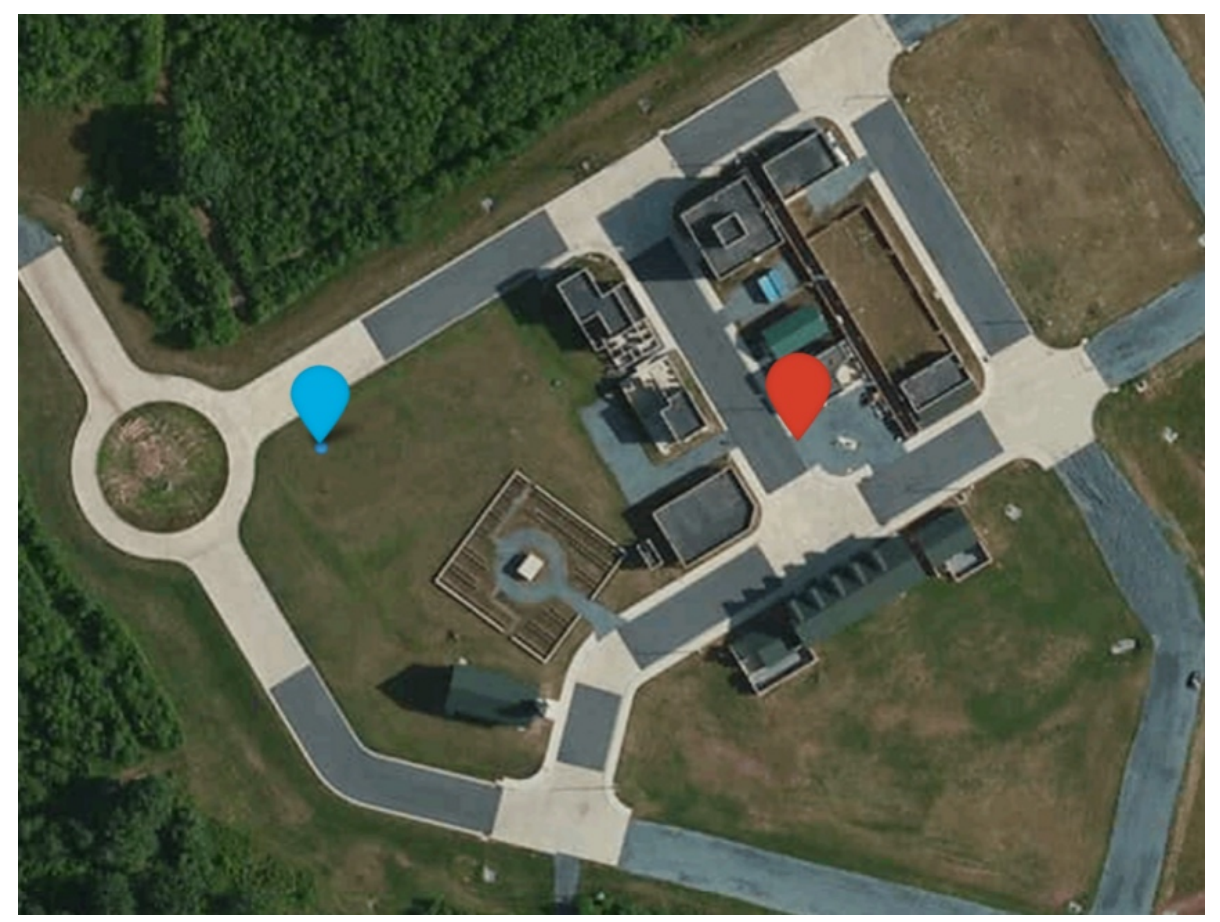

Figure 4.6.2. Persistent weather monitoring station locations.

As with the OPTUS 1 and 2 measurements, the goal of these measurements was to compliment the measurement of gamma-ray backgrounds at the CACTF by monitoring area radiation with a persistent detector system and using it as a reference for anomalies. Both WPRM stations were fully set up and connected to the data acquisition system around 1:00 p.m. on the first day of campaign. The WPRM-1 station successfully ran for the entire time during the campaign and collected total of $40 \mathrm{~h}$ of data. On the other hand, the WPRM-2 ran only $18 \mathrm{~h}$ before it ran out of main battery power, and the backup battery was not delivered as a result of logistical errors. 
One of the main goals of the measurements with ORNL WPRM stations was to provide an independent reference for main radiation measurements at the range, such as static measurements at the Main St. For the MUSE 1 campaign, static Main St. measurements were performed in two separate takes on Day 2 of the campaign approximately $3 \mathrm{~h}$ apart. Static background spectra measurements at position markers with even $x$-coordinates were performed between 12:00-2:00 p.m. and at position markers with odd were performed between 4:00-6:00 p.m. A side-by-side comparison of the count rates between these two measurements, shown in Figure 4.6.3, reveals $\sim 1.5 \%$ systematic difference in total gamma-ray spectra counts per second between these two sets of measurements.
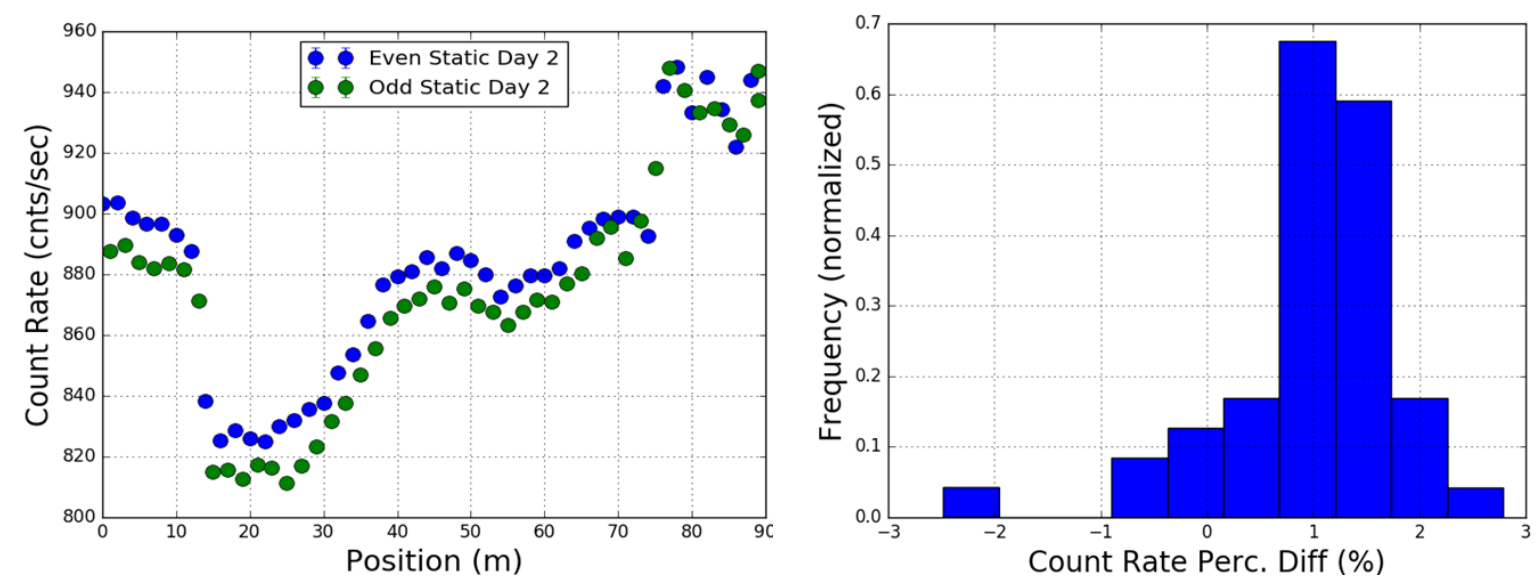

Figure 4.6.3. MUSE 1 static measurement comparison along Main St. (Left) Static background comparison along Main St. for different times of the day and (right) the count rate difference between measurement times.

Separately, full spectra counts per second recorded by persistent radiation monitoring detector as a function of time, shown in Figure 4.6.4, also shows a decrease by approximately the same percentage amount between the times corresponding to these two sets of measurements. This independent measurement confirms that radiological conditions changed slightly between these time periods. 


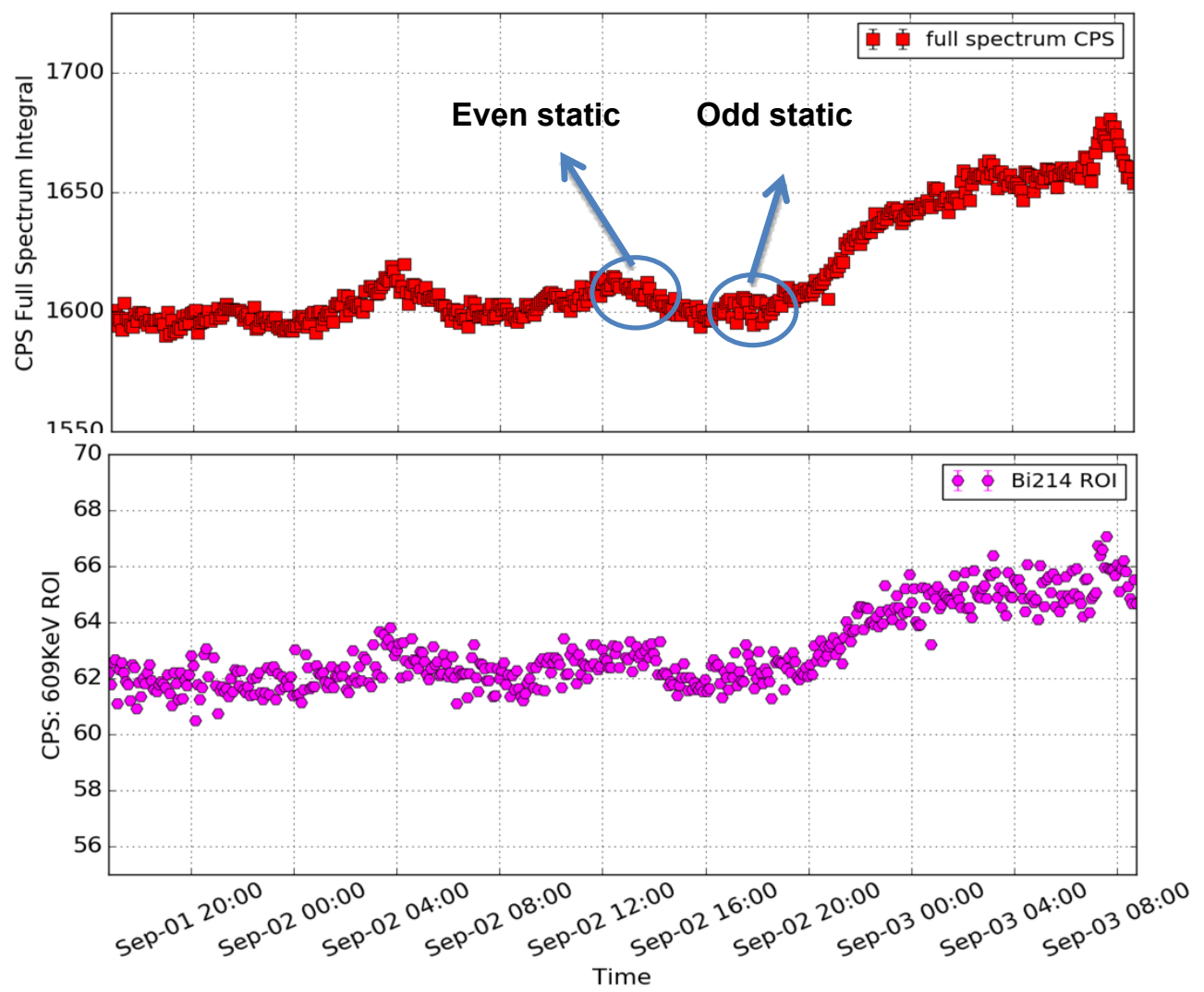

Figure 4.6.4. (Above) Persistent background monitor gross count rates versus time and (below) count rates versus time for the ${ }^{214} \mathrm{Bi}$ ROI.

The lower portion of Figure 4.6.4, shows the time-dependent counts per second integral within $609 \mathrm{keV}$ for an ROI with an almost identical trend as the full spectra counts per second as function of time distribution. On the other hand, Figure 4.6.5 shows counts per second integrals within ${ }^{40} \mathrm{~K}$ and ${ }^{232} \mathrm{Th}$ ROIs in the time interval between 12:00 p.m. and 6:00 p.m. and does not reveal any change in detected levels of ${ }^{40} \mathrm{~K}$ or ${ }^{232} \mathrm{Th}$.

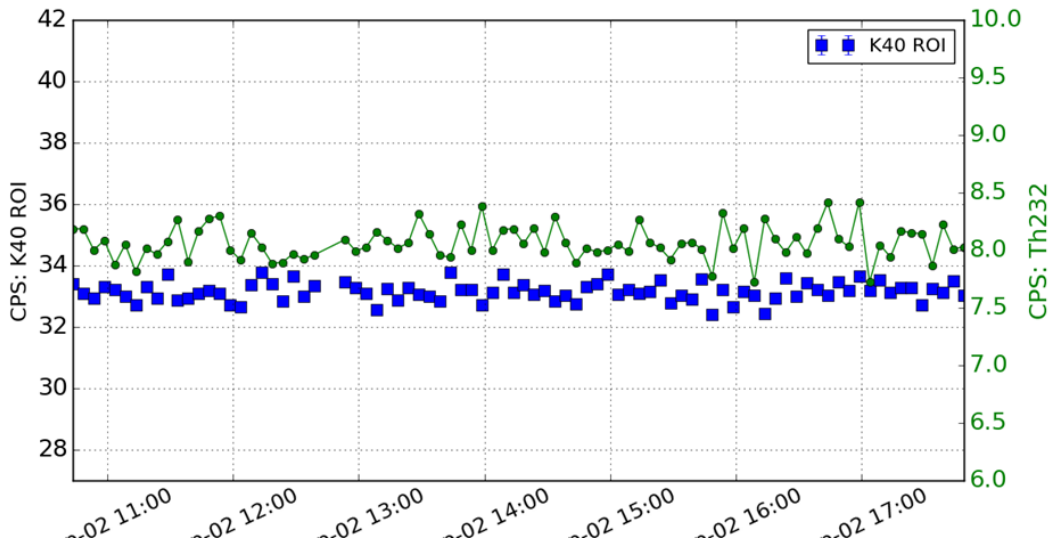

Figure 4.6.5. Count rates versus time for ${ }^{40} \mathrm{~K}$ ROI and ${ }^{232} \mathrm{Th}$ ROI.

To establish the environmental event(s) or observable(s) that may be responsible for the observed gammaray variations, each weather variable is overlaid with spectral information in Figure 4.6.6. 


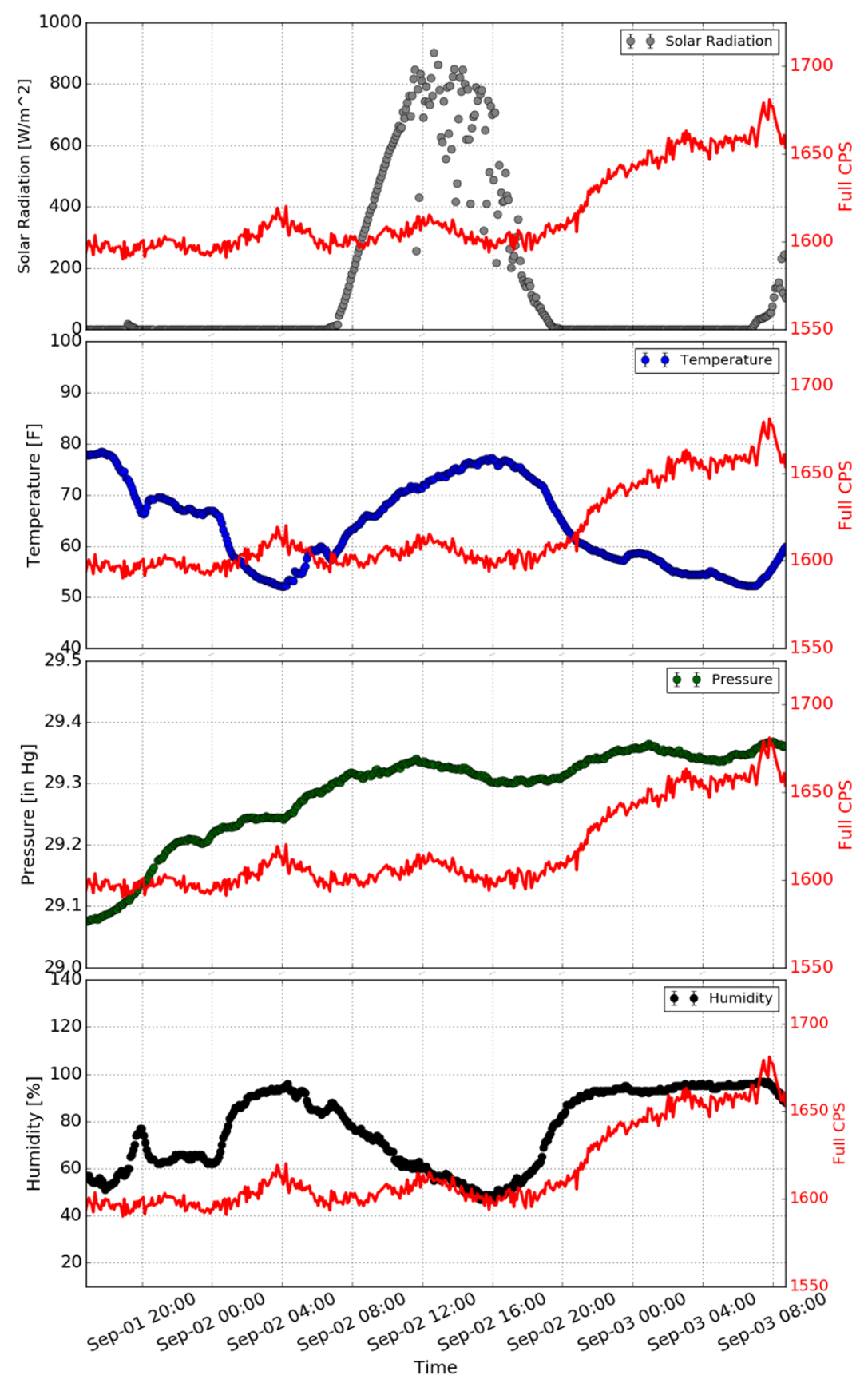

Figure 4.6.6. Comparison of environmental variables and count rate over time for persistent monitoring station.

Persistent radiation monitoring data compared to key weather observables as a function of time reveals no obvious correlation with one single weather variable. However, it hints that there is a correlation of complicated multivariate nature. Scatter plots, shown in Figure 4.6.7, provide no obvious correlation to the radiation counts observed, but trends seem to be visible for temperature, pressure, and humidity. On this short time scale, the change in gamma background appears to coincide with the change in several weather variables. The observed correlation, however, is nontrivial and further studies are underway to analyze these effects over a large time scale. 

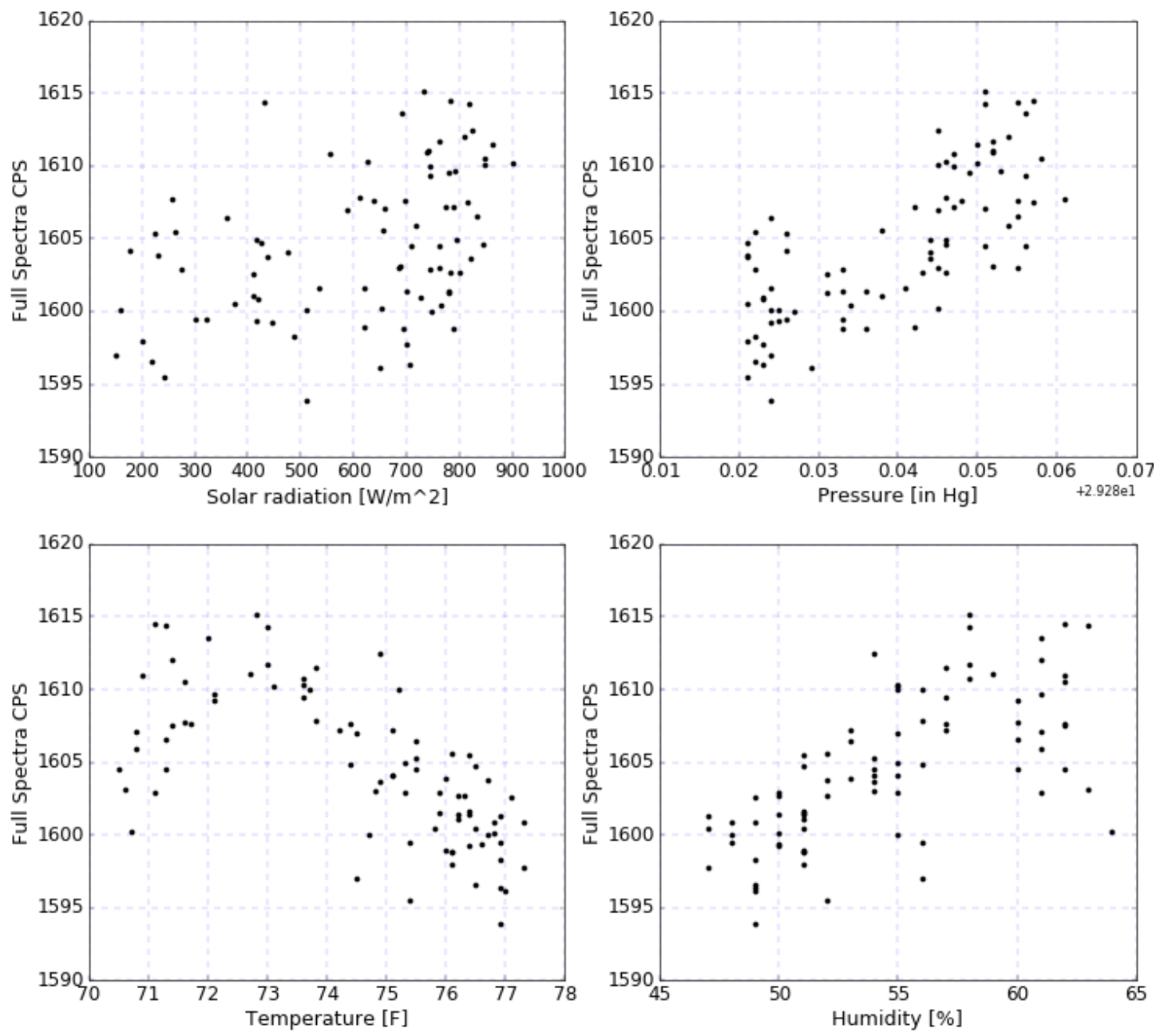

Figure 4.6.7. Count rate versus weather variables between 12:00 p.m. and 6:00 p.m. on September 2.

\subsection{RadMAP MEASUREMENTS}

The Radiological Multi-sensor Analysis Platform (RadMAP) is a multisensor system fielded on a $30 \mathrm{ft}-$ long truck. A schematic of the system is shown as Figure 4.7.1. RadMAP has one hundred 2 in. $\times 4$ in. $\times$ 4 in. $\mathrm{NaI}(\mathrm{Tl})$ detectors that are positioned in a $10 \times 10$ array behind a lead coded mask, 14 mechanically cooled HPGe detectors, and 16 EJ-309 liquid scintillator detectors. All these radiation sensors are configured to "view" out the starboard side of the truck's trailer, although there is no shielding beyond detector readout systems to prevent detection of radiation incident from the port side. For contextual sensors, there are three additional starboard-facing cameras: a charge coupling device (CCD) camera for image overlay and two hyperspectral imagery (HSI) cameras, which are sensitive to wavelengths of 400 $900 \mathrm{~nm}$ and $900-1,700 \mathrm{~nm}$. Mounted on the front of the truck trailer are two $3 \pi$ Ladybug camera systems, two 32-beam Velodyne LIDAR systems, a weather station, and an antenna for the GPS/IMU system. The LIDAR and Ladybug cameras are mounted on the front corners to provide good imagery coverage of both sides of the system. A more thorough description of the RadMAP system can be found in Ref. [11].

RadMAP collections are summarized in Section 4.7; an overview of the RadMAP radiation detector findings is provided in Subsections of 4.7.1; the results of the weather data are provided in Section 4.7.2; the anomaly algorithms and an off-site anomaly encounter are described in Section 4.7.3; the results of system localization and mapping using various contextual sensors is described in Section 4.7.5; a 
comparison between RadMAP radiological data and those obtained with the sport utility vehicle (SUV)borne RSI system is provided in Section 4.7.6; a brief overview of RadMAP analyses discussing the efforts of combining radiological and contextual data is provided in Section 4.7.7; and finally, Section 4.7.8 summarizes the status of analyses performed on the HSI data.
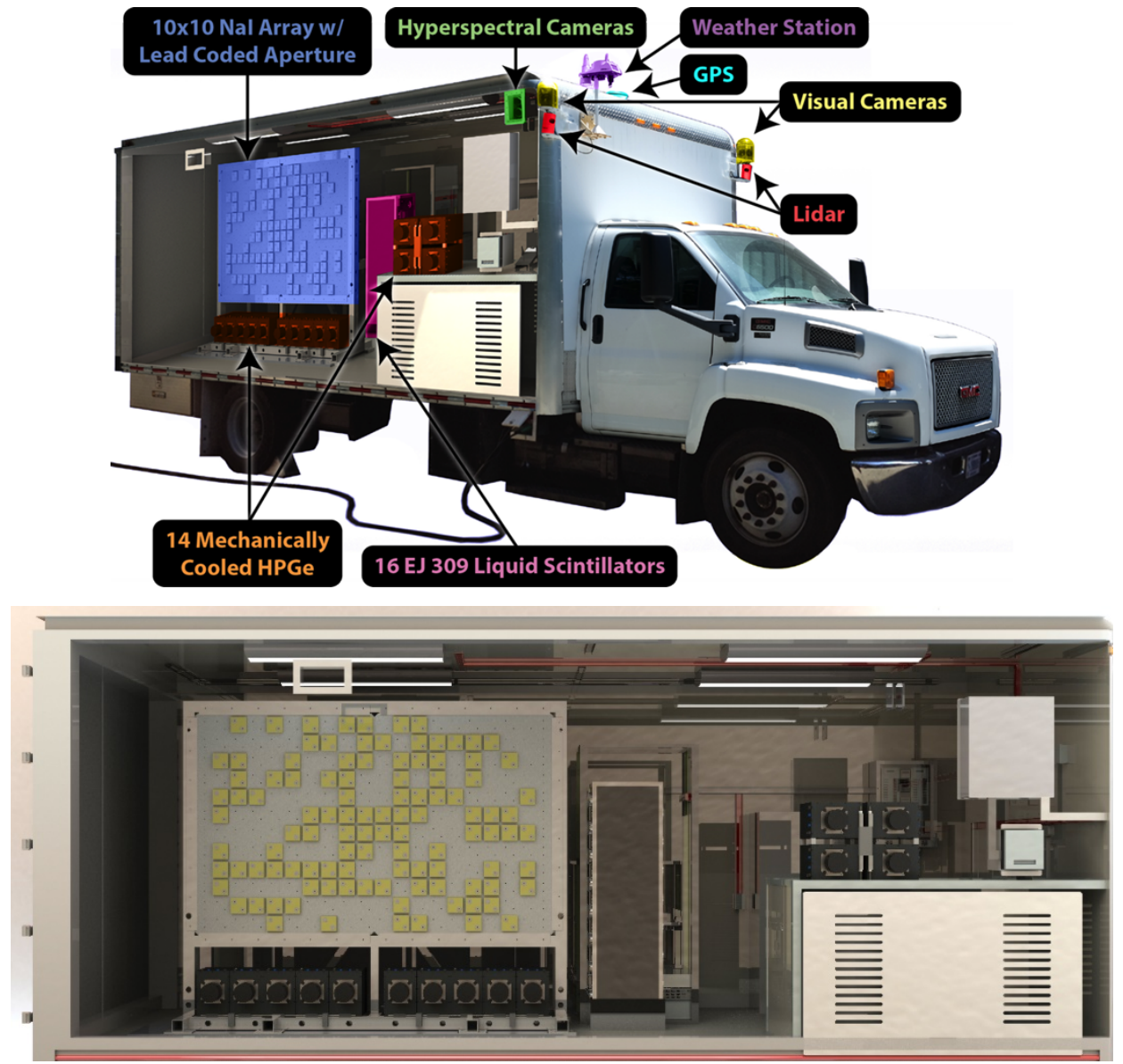

Figure 4.7.1. Illustration of RadMAP system with an overlaid drawing indicating the positions of the fielded sensors.

The RadMAP system performed data collections over September 1-3, 2016. The collections included $3 \mathrm{~h}$ of dynamic collections with NA-84 RSI mobile system following for comparison. Dynamic runs were made at 5, 10, and $15 \mathrm{mph}$ on loops through Main St. One hour of static measurements on Church St. were completed. The RadMAP team conducted four HSI collections against all roads on Range 30. Ten hours of static measurements on Main St. were completed. Additional dynamic runs on Main St. with clutter vehicles and an accompanying NA-84 mobile system following for comparison were made. A summary of measurements is provided in Table 4.7.1. 
Table 4.7.1. A summary of RadMAP measurements performed during MUSE-1 campaign.

\begin{tabular}{rlccll}
\hline Date & $\begin{array}{c}\text { Dynamic } \\
\text { meas. }\end{array}$ & $\begin{array}{c}\text { RSI } \\
\text { trailing }\end{array}$ & $\begin{array}{c}\text { Static } \\
\text { meas. }\end{array}$ & \multicolumn{1}{c}{ HSI survey } & Additional Comments \\
\hline \hline $9 / 1 / 2016$ & 90 laps & 49 laps & 3 & $\begin{array}{l}1: 00 \text { p.m., mixed clouds } \\
\text { full-site survey }\end{array}$ & \\
$9 / 2 / 2016$ & $\begin{array}{l}\text { 4 HSI site } \\
\text { surveys }\end{array}$ & - & $39+2$ & $\begin{array}{l}\text { Sunny, full-site surveys } \\
\text { at 9:00 a.m., 1:00 p.m., } \\
3: 00 \text { p.m., and 6:00 p.m. }\end{array}$ & $\begin{array}{l}\text { Provided "vehicle clutter" for UAV } \\
\text { measurements. Static measurements } \\
\text { of field with and without obstructing } \\
\text { vehicle. }\end{array}$ \\
$9 / 3 / 2016$ & 46 laps & 15 laps & 3 & Sunny morning surveys & $\begin{array}{l}\text { Added parked vehicles on Main St. } \\
\text { after site surveys. Performed } \\
\text { dynamic measurement clutter } \\
\text { experiments. }\end{array}$ \\
\hline
\end{tabular}

A top-down view of the path of the first 52-lap survey conducted by RadMAP on September 1 is overlaid on satellite imagery in the left side of Figure 4.7.2. Also shown on the right side of the figure is a 3-D perspective view of the surveys, where the $z$-dimension and coloring indicate time. This 52-lap survey took $60 \mathrm{~min}$. to complete. During this survey, an SUV-borne RSI system trailed the RadMAP system beginning after the second lap. The first set of laps were oriented to go uphill along Main St. at nominal speeds of 5, 10, and $15 \mathrm{mph}$ for 10 laps at $5 \mathrm{mph}$ and to go 7 laps at 10 and $15 \mathrm{mph}$. These loops were clockwise around loop A. Afterward, five downhill, counter-clockwise $5 \mathrm{mph}$ laps along loop A were conducted, with RadMAP still in the west lane, which is equivalent to measurements in the left-lane of a one-way road. Observed speed profiles along Main St. for these collections are shown in Figure 4.7.3. Next, RadMAP collected 23 downhill (clockwise) loop B laps at 5, 10 and $15 \mathrm{mph}$ followed by 10 counter-clockwise loop B laps. The team then conducted six counter-clockwise laps of the exterior loop of the facility at $10 \mathrm{mph}$. After lunch, the team repeated the uphill loop A measurements at 10 then $5 \mathrm{mph}$ with fewer vehicles on Main St. The team proceeded to perform another 12 counter-clockwise outer loops of the facility, followed by three static measurements along Church St. (the southern street) (Figure 4.7.4). The entirety of the day's activities is logged in Table 4.7.2. Times are listed in Pacific daylight time (PDT).
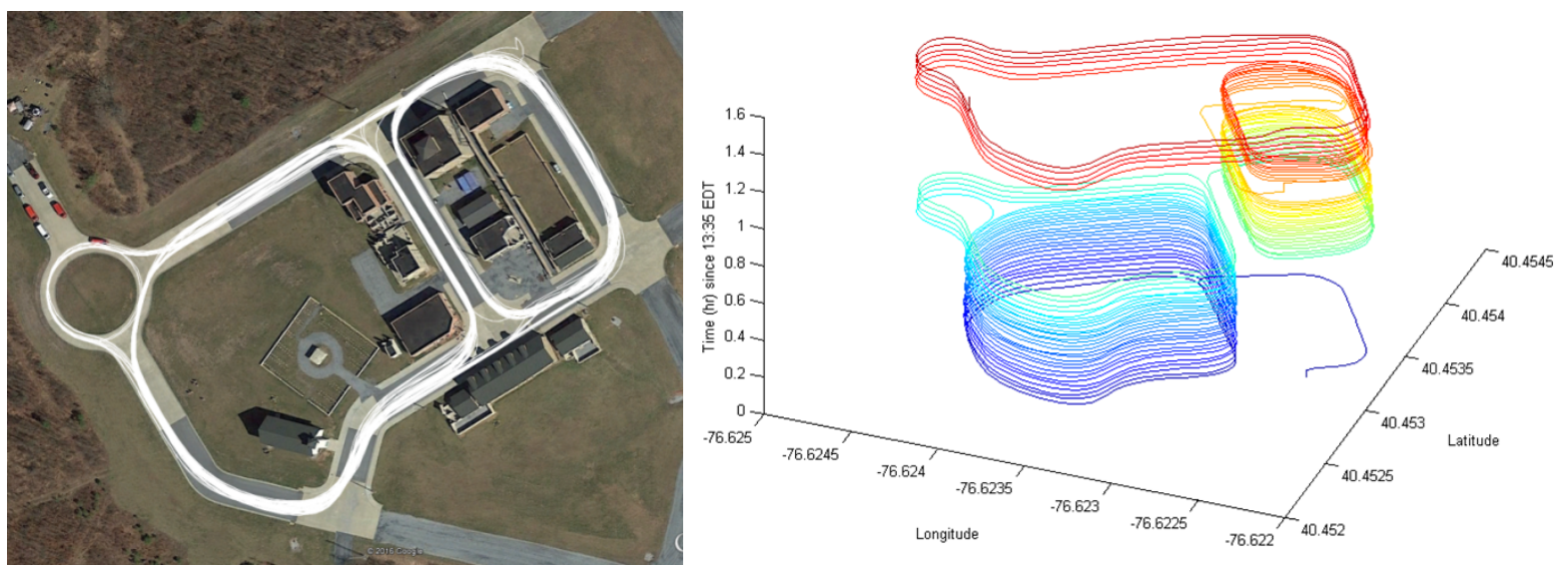

Figure 4.7.2. RadMAP dynamic measurements through FTIG. (Left) Top-down view of RadMAP Day 1 survey paths. (Right) Perspective view of corresponding survey where the $z$-dimension and coloring represent time. 


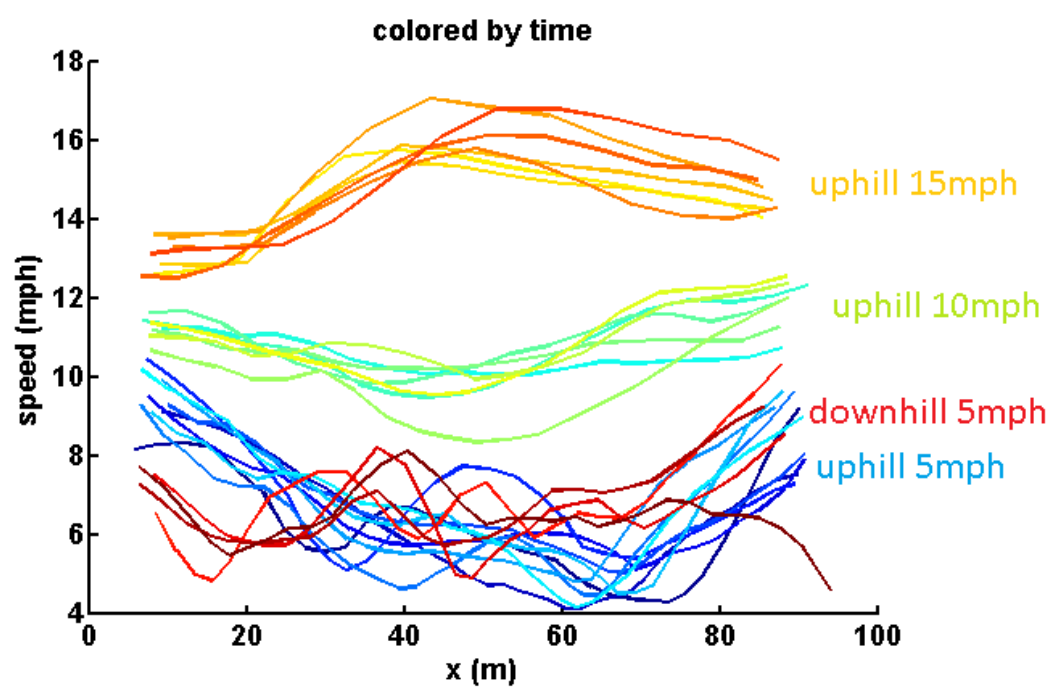

Figure 4.7.3. RadMAP Speed profiles along Main St. for initial 29 loops. The curves are colorized by relative time, with the first 10 uphill nominal $5 \mathrm{mph}$ loops in blue, then seven $10 \mathrm{mph}$ loops in green-yellow, then uphill $15 \mathrm{mph}$ in yellow/red, followed by the downhill $5 \mathrm{mph}$ loops in red. The abscissa is linear distance along Main St., relative to a point approximately at the center of the northern intersection.

Figure 4.7.4 indicates the positions of the static measurements taken by RadMAP during Days 1 and 2 . For all static measurements, the truck was oriented so that the starboard side was facing the nearest building, as would be expected during operation on a typical two-way street. Each static measurement was at least $10 \mathrm{~min}$. in duration, but due to other ongoing measurement activities, RadMAP was on some occasions asked to not move, resulting in some measurements extending longer. Time-perspective summaries of Days 2 and 3 RadMAP paths are shown as Figure 4.7.5.
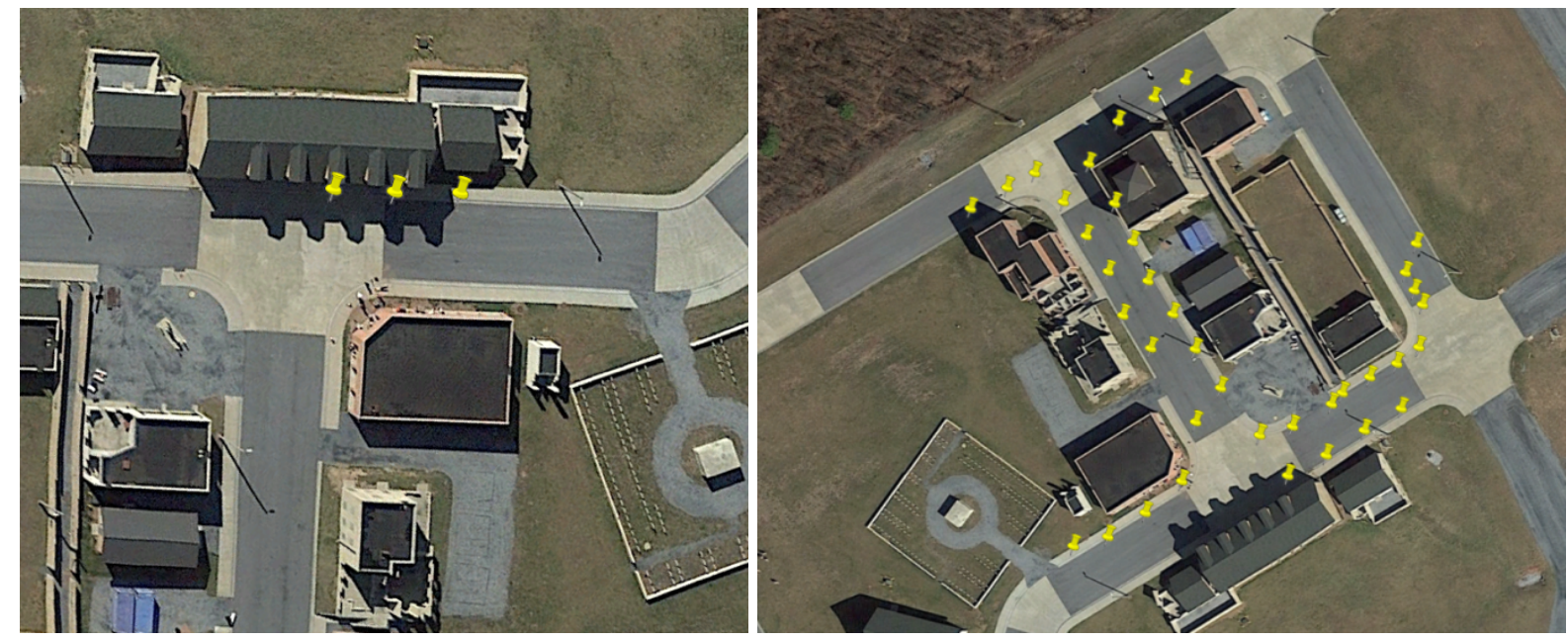

Figure 4.7.4. RadMAP static measurement locations. (Left) Locations of Day 1 static measurements. (Right) Day 2 static measurement positions. 
Table 4.7.2. Summary of RadMAP Day 1 collections.

\begin{tabular}{ccccccccc}
\hline Start & Stop & $\begin{array}{c}\text { Speed } \\
\text { (mph) }\end{array}$ & Main St. & Dir. & Loop & Side & No. & Notes \\
\hline \hline $10: 39$ & $10: 52$ & 5 & Uphill & CW & A & R & 10 & Some cars parked on Main St. \\
$10: 52$ & $10: 59$ & 10 & Uphill & CW & A & R & 7 & Pass 5, closer to 8 mph \\
$10: 59$ & $11: 05$ & 15 & Uphill & CW & A & R & 7 & - \\
$11: 07$ & $11: 14$ & 5 & Downhill & CCW & A & L & 5 & Added ORNL RUNE system, \\
$11: 14$ & $11: 21$ & 5 & Downhill & CW & B & R & 8 & - \\
$11: 22$ & $11: 27$ & 10 & Downhill & CW & B & R & 7 & - \\
$11: 27$ & $11: 33$ & 15 & Downhill & CW & B & R & 8 & 1st, was off, b/t 3\&4 was \\
$11: 38$ & $11: 42$ & 5 & Uphill & CCW & B & L & 5 & mph \\
$11: 43$ & $11: 46$ & 10 & Uphill & CCW & B & L & 5 & - \\
$11: 47$ & $11: 56$ & 10 & N/A & CCW & Outer & R & 6 & - \\
$12: 30$ & $12: 33$ & 10 & Uphill & CW & A & R & 5 & - \\
$12: 33$ & $12: 40$ & 5 & Uphill & CW & A & R & 5 & - \\
$13: 00$ & $13: 10$ & 5 & N/A & CCW & Outer & R & 7 & Van moved after pass 3 3 wassed" \\
$13: 11$ & $13: 17$ & 10 & N/A & CCW & Outer & R & 5 & - \\
$13: 17$ & $14: 11$ & 0 & N/A & N/A & N/A & R & 3 & Static measurements along \\
\hline
\end{tabular}

*Times in Pacific daylight time (PDT).
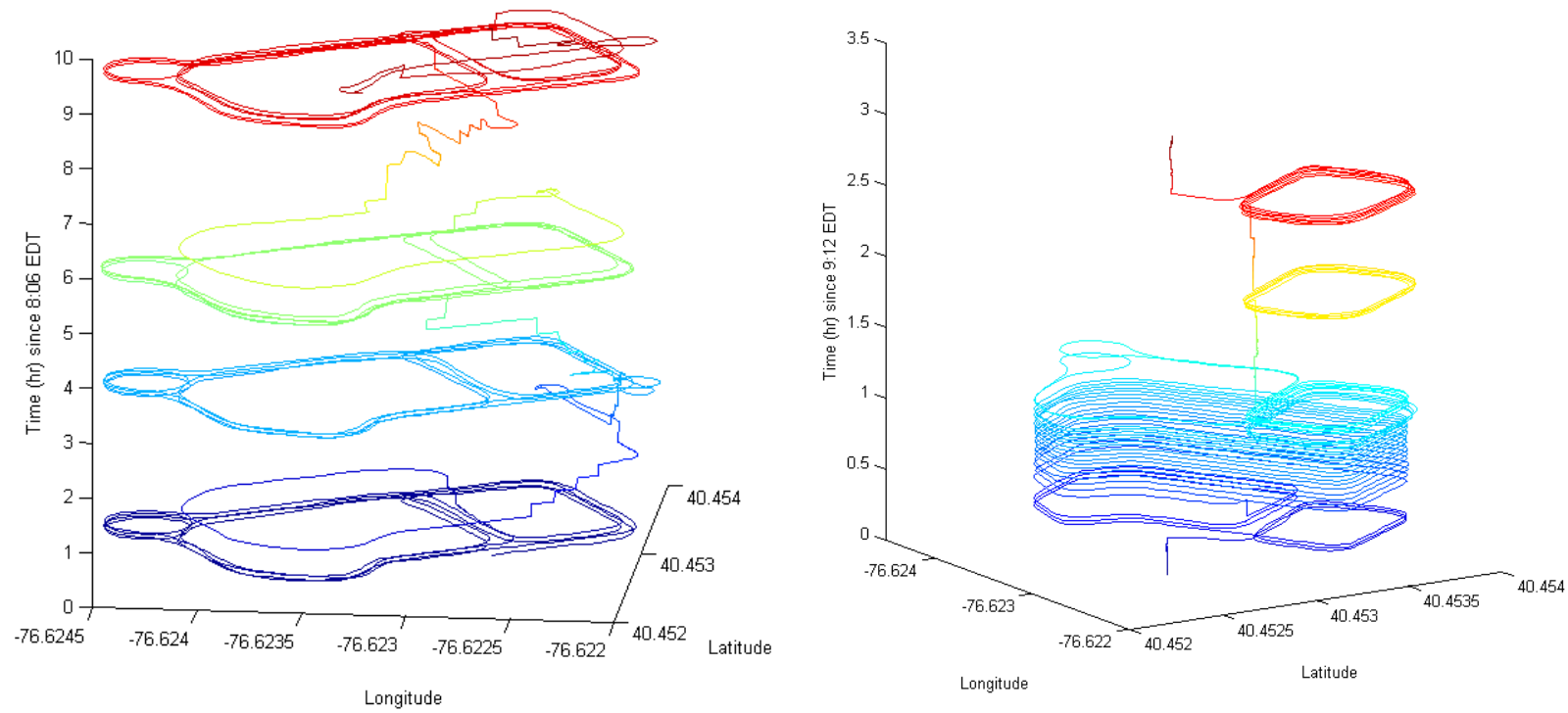

Figure 4.7.5. RadMAP loops through FTIG. (Left) RadMAP Day 2 time-perspective path. (Right) RadMAP Day 3 time-perspective path. 
During Day 2, RadMAP concentrated on performing static measurements, primarily while not interfering with other ongoing measurement activities, particularly on Main St. The locations of the static measurements are indicated in the right image of Figure 4.7.4 and are summarized in Table 4.7.3. RadMAP also performed full-site HSI surveys at 8:00 a.m., 11:00 a.m., and 4:00 p.m. local time. For each of these surveys, RadMAP HSI cameras observed each façade at least twice. Lastly, RadMAP also participated in a drive-around near 1:00 p.m. local time for the purpose of providing moving clutter for UAV-collected imagery. During these measurements, additional HSI and survey data were collected. At the end of Day 2, the RadMAP system was left operating as the team returned to the hotel. On entering the hotel parking lot, large ${ }^{137} \mathrm{Cs}$ anomaly alarms were triggered. These are described in Section 4.7.4.

Day 3 measurements included completing additional outer loop measurements, performing the final three static measurements at locations on Main St., and conducting an extra set of 31 clutter measurement surveys that were unplanned. These measurements were all performed at nominal $10 \mathrm{mph}$ speeds, with RadMAP driving on the right side of Main St. in both the uphill and downhill directions. The first measurements were low clutter and no vehicles were parked on Main St. For the medium clutter measurements, a cargo van and a Jeep were parked on the west side of Main St., near the north end of block. After completion of the medium clutter measurements, the faux fire hydrant and the Jeep were both moved (approximately $10 \mathrm{~cm}$ and $1 \mathrm{~m}$, respectively) and the loop A medium clutter surveys were repeated. Finally, a total of five vehicles were parked on Main St. and five high clutter loop A

measurements were conducted. The Day 3 RadMAP measurements are summarized in Table 4.7.4. At the end of the campaign, RadMAP was left operational when driven offsite and was parked two parking spots away from the ${ }^{137} \mathrm{Cs}$-laden vehicle.

Table 4.7.3. Summary of RadMAP Day 2 collections.

\begin{tabular}{|c|c|c|c|c|c|c|c|c|c|}
\hline Start & Stop & \multirow{2}{*}{\multicolumn{3}{|c|}{$\begin{array}{cc}\text { Location } & \text { Notes } \\
2 \times \text { entire site HSI survey }\end{array}$}} & Start & Stop & Location & & Notes \\
\hline $5: 13$ & $5: 24$ & & & & $10: 22$ & $10: 33$ & $\mathrm{RH}$ & 2 & - \\
\hline & & \multicolumn{3}{|c|}{$\begin{array}{r}2 \times \text { entire site HSI survey } \\
\text { RME }=\text { RadMAP }\end{array}$} & & & & & \\
\hline $5: 28$ & $5: 38$ & RME & 5 & $\ldots$ & $10: 38$ & $10: 48$ & $\mathrm{RP}$ & 1 & Police station \\
\hline $5: 39$ & $5: 49$ & RME & 6 & Main st, E side" & $10: 49$ & $10: 59$ & $\mathrm{RP}$ & 2 & - \\
\hline $5: 53$ & $6: 04$ & $\mathrm{RC}$ & 4 & $\mathrm{RC}=$ Church St. & $11: 26$ & $11: 37$ & $\mathrm{RP}$ & 3 & - \\
\hline $6: 05$ & $6: 15$ & $\mathrm{RC}$ & 5 & - & $11: 40$ & $11: 50$ & RMW & 5 & Main St. west side \\
\hline $6: 17$ & $6: 27$ & $\mathrm{RC}$ & 6 & - & $11: 54$ & 12:04 & RMW & 6 & RaspberryPi CCD crashed \\
\hline $6: 28$ & $6: 38$ & $\mathrm{RC}$ & 7 & - & $12: 06$ & $12: 16$ & RMW & 7 & - \\
\hline $6: 39$ & $6: 49$ & \multicolumn{3}{|c|}{ Utility truck field } & $12: 18$ & $12: 30$ & RMW & 3 & - \\
\hline $6: 50$ & $7: 01$ & \multicolumn{3}{|c|}{ No utility truck, field } & $12: 32$ & $12: 43$ & RMW & 2 & - \\
\hline $7: 03$ & $7: 13$ & $\mathrm{RR}$ & 1 & $\mathrm{RR}=$ Restaurant & $12: 45$ & $12: 55$ & RMW & 1 & - \\
\hline $7: 15$ & $7: 27$ & $\mathrm{RR}$ & 2 & - & $12: 57$ & 13:08 & $\mathrm{RME}$ & 1 & - \\
\hline $7: 28$ & $7: 50$ & $\mathrm{RR}$ & 3 & - & $13: 10$ & $13: 21$ & RME & 7 & - \\
\hline $7: 50$ & $8: 00$ & \multicolumn{3}{|c|}{ HSI Survey } & $13: 32$ & $13: 46$ & \multicolumn{3}{|c|}{$2 \times$ entire site HSI survey } \\
\hline $8: 03$ & $8: 13$ & $\mathrm{RR}$ & 5 & - & $13: 47$ & $13: 57$ & RA & 2 & Alleyway \\
\hline $8: 16$ & $8: 26$ & $\mathrm{RR}$ & 6 & - & $13: 59$ & $14: 12$ & $\mathrm{RB}$ & 4 & - \\
\hline $8: 28$ & $8: 38$ & $\mathrm{RR}$ & 7 & - & $14: 19$ & $14: 29$ & RSS & 2 & - \\
\hline $8: 39$ & $8: 49$ & $\mathrm{RR}$ & 8 & - & $14: 31$ & $14: 41$ & RMM & & - \\
\hline $8: 51$ & $9: 01$ & RA & 1 & Alleyway & $14: 43$ & $14: 53$ & $\mathrm{RB}$ & 1 & - \\
\hline $9: 05$ & $9: 20$ & RSS & 1 & Service station & $14: 56$ & 15:06 & RME & 2 & - \\
\hline $9: 22$ & $9: 32$ & $\mathrm{RB}$ & 3 & Bar & $15: 08$ & $15: 18$ & RME & 3 & Lots of activity near truck \\
\hline $9: 33$ & $9: 55$ & $\mathrm{RB}$ & 2 & - & $15: 19$ & $15: 29$ & RME & 4 & - \\
\hline
\end{tabular}




\begin{tabular}{cclc|cll} 
9:56 & 10:06 & \multicolumn{2}{l|}{ Clutter driving to provide } & $15: 31$ & $14: 46$ & RMW 4 \\
& & UAS dynamic clutter & $15: 46$ & $16: 05$ & Return to hotel \\
10:09 & $10: 19$ & RH $\quad 1$ & hotel & $16: 05$ & $16: 10$ & Identified 137Cs in hotel lot \\
\hline
\end{tabular}

*Times in Pacific daylight time (PDT).

Table 4.7.4. Summary of RadMAP Day 3 collections.

\begin{tabular}{cccccccll}
\hline Start & Stop & $\begin{array}{c}\text { Speed } \\
(\mathbf{m p h})\end{array}$ & Main St. & Dir. & Loop & Side & No. & Notes \\
\hline \hline $6: 25$ & $6: 29$ & 10 & Downhill & CW & B & R & 5 & Low clutter \\
$6: 29$ & $6: 36$ & 10 & Uphill & CW & A & R & 5 & Low clutter \\
$6: 43$ & $6: 55$ & 5 & N/A & CW & Outer & R & 5 & - \\
$6: 55$ & $7: 05$ & 10 & N/A & CW & Outer & R & 5 & - \\
$7: 05$ & $7: 12$ & 15 & N/A & CW & Outer & R & 5 & - \\
$7: 12$ & $7: 17$ & 10 & Uphill & CW & A & R & 5 & Medium clutter \\
$7: 17$ & $7: 22$ & 10 & Downhill & CW & B & R & 6 & Medium clutter \\
$8: 06$ & $8: 09$ & 10 & Uphill & CW & A & R & 5 & Shifted fire hydrant and Jeep \\
$8: 46$ & $8: 51$ & 10 & Uphill & CW & A & R & 5 & High clutter \\
$8: 52$ & $9: 29$ & 0 & N/A & N/A & N/A & R & 3 & Three static measurements on \\
\hline
\end{tabular}

*Times in Pacific daylight time (PDT).

The position of the RadMAP truck during each static measurement was determined by dividing each segment of roadway into the fewest number of positions that would yield less than $10 \mathrm{~m}$ regular spacing between measurement positions. The positions were then marked on the roadway, and RadMAP was positioned so that the front of the access steps was aligned to within an inch of the marking. RadMAP was also positioned to the right side of the roadway so that the tires were resting on the curb piece of roadway, where present. Otherwise, the truck was within $10 \mathrm{~cm}$ of the right side of the pavement. Figure 4.7.6 provides several photographs indicating RadMAP positioning.

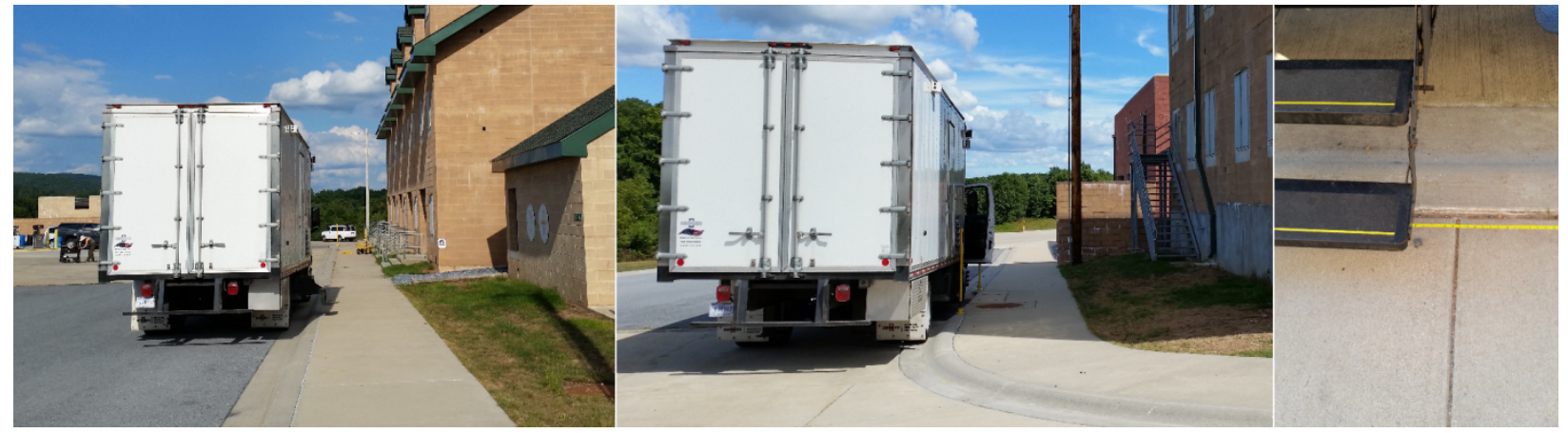

Figure 4.7.6. Photograph of RadMAP static measurement process. (Left) Measurement RC1, (center) measurement RH1, (left) photograph of step being measured for positioning. The left and center photographs are indicative the left-right range of RadMAP positioning relative to the roadway curbs (where present).

\subsubsection{RadMAP Radiological Data}

RadMAP operated ninety-nine 2 in. $\times 4$ in. $\times 4$ in. NaI(Tl) detectors, 13 HPGe interchangeable detector modules (IDM) detectors, and 16 1.5-L EJ-309 liquid scintillators (LS) for fast neutron detection. The 
radiation detection systems were predominantly stable throughout the course of measurement campaign, although the liquid scintillators appeared to become unstable and shut off after many hours of measurements. This will be discussed in more detail below. A detailed view of the RadMAP interior is shown as Figure 4.7.6. The $\mathrm{NaI}(\mathrm{Tl})$ detectors are arranged in a $10 \times 10$ array where the 4 in. $\times 4$ in. crystal faces are pointed horizontally, perpendicular to the direction of travel. The array has a 1 in.-thick lead coded mask positioned starboard of the array, and each crystal is read-out by photo-multiplier tubes mounted on the port face of the crystals. The dimension of the $\mathrm{NaI}(\mathrm{Tl})$ array is approximately $1 \mathrm{~m} \times 1 \mathrm{~m}$ and the lead mask's dimensions are $1.8 \mathrm{~m} \times 1.2 \mathrm{~m}$. The $\mathrm{NaI}(\mathrm{Tl})$ array is centered in the longer horizontal direction of the lead mask, and the bottoms of the arrays are aligned. Nine IDM detectors (numbered 0-9, but the first one malfunctioned and did not join the campaign) are positioned near the floor of the RadMAP truck, beneath the lead mask array and on top of a layer of 2 in. thick lead. The lead is 8 in.wide and extends the entire $\sim 1.8 \mathrm{~m}$ length of the IDM floor array. The IDMs were all positioned approximately $2.5 \mathrm{ft}$ from the starboard wall of RadMAP and are facing starboard. An additional four IDM detectors (numbered 10-13) are arranged in a $2 \times 2$ configuration atop the generator compartment closer to the front of the truck. These detectors also face starboard, but they nearly about the starboard wall of RadMAP. The EJ-309 detectors are oriented horizontally facing starboard and are stacked vertically in two columns of eight detectors each. Each detector is a 5 in. diameter by 5 in.-long packaged in an aluminum cylinder and coupled to a photo-multiplier tube on the port end of the cylinder. The LS array is positioned to the fore of the lead coded mask. To the port of the LS array is the computer rack that contains the main data acquisition and storage servers. A schematic of the detector arrangement is provided in Figure 4.7.1; however, one of the IDMs was not fielded for the MUSE-1 campaign due to malfunction. All radiation detectors are read-out and integrated using VME-based Struck digitizers. Listmode energy and time data are stored for the gamma-ray detectors and digitized waveforms are stored for the LS detectors.

\subsubsection{Dynamic Gamma-ray Detector Measurements}

An example $\mathrm{NaI}(\mathrm{Tl})$ array spectrum is provided in the left side of Figure 4.7.7. The data are summed across all 99 operational detectors, and were binned into $3 \mathrm{keV}$-wide energy bins. The measurement is for the first approximately $3 \mathrm{~h}$ of data from Day 1 . The observed $80 \mathrm{keV} \mathrm{FWHM} \mathrm{energy} \mathrm{resolution} \mathrm{at}$ $1,460 \mathrm{keV}$ is consistent with historical RadMAP NaI(Tl) array performance of $8.1 \% \mathrm{FWHM}$ at $662 \mathrm{keV}$. Inspecting individual detectors indicated there is some variability in individual $\mathrm{NaI}(\mathrm{Tl})$ detector performance, but no detector stands out as an outlier. Likewise, variable energy calibrations do not appear to be contributing to the observed energy resolution of the summed array.
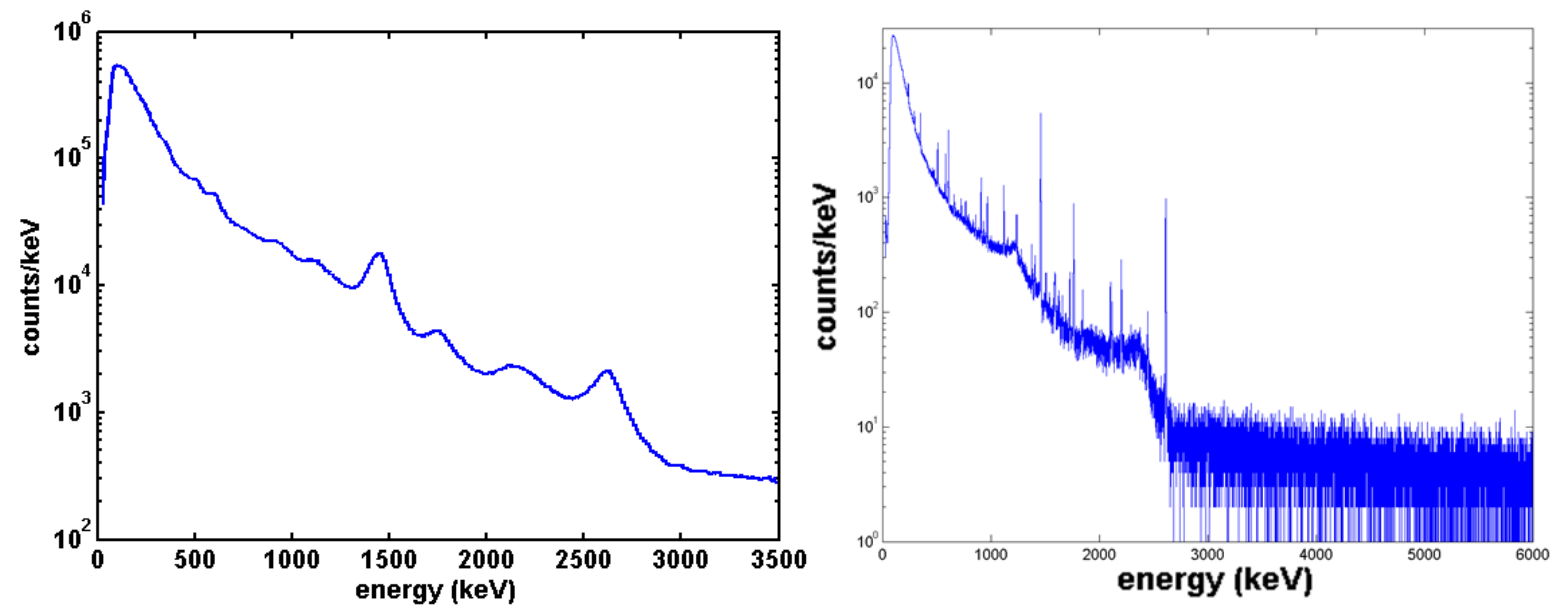

Figure 4.7.7. Example 3 h dynamic RadMAP spectrum collected Day 1. (Left) RadMAP summed NaI(TI) array spectrum from $\sim 3 \mathrm{~h}$ of Day 1 dynamic measurements.(Right) Same data for IDM array. 
The summed IDM array spectrum is provided in the right side of Figure 4.7.7, each energy bin is $1 \mathrm{keV}-$ wide. Clearly, the IDM array enables resolution of significantly more individual discrete gamma-ray transitions. Despite this, the energy resolution of the IDM array is at present limited by the BNC readout system, which easily integrates into the RadMAP data acquisition system, but bypasses the IDM active noise dampening circuitry, resulting in worse energy resolution than could be otherwise achieved. The $\mathrm{NaI}(\mathrm{Tl})$ array generates a spectrally integrated count rate that is approximately 12 times that of the IDMs. The IDMs performed quite well although on Day 1, two detectors (numbers 2 and 13) showed intermittent low-energy noise, most likely associated with the cooling system. The effects of the noise could be removed during Day 1 by only considering registered events of greater than $70 \mathrm{keV}$. During Day 2, the performance of detector 13 deteriorated, and the detector ceased functionality during the runs. This detector was omitted from Days 2 and 3.

Of particular interest is the radiological variability that was observed across the site. The four plots in Figure 4.7.8 indicate, as a function of position and time (the vertical axis) for the morning loops (e.g., 10:39-11:33) in the Day 1 measurements, the gross count rate (top left), the ${ }^{40} \mathrm{~K}$ peak rate (top right), the uranium-series ROI rate (bottom left), and the ${ }^{208} \mathrm{Tl}$ peak rate (bottom rate), each integrated into $1 \mathrm{~s}$ intervals for the $\mathrm{NaI}(\mathrm{Tl})$ array. Although determining the gross count rate is self-explanatory, describing the derivation of the other rates is worth describing in more detail. The peak rate of ${ }^{208} \mathrm{Tl}$ is determined by summing the energy region from 2450 to $2,800 \mathrm{keV}$ after subtracting a sum attributed to the cosmic continuum. The cosmic continuum is estimated by first fitting the energy region above $3,050 \mathrm{keV}$ as a power series. Then, it is assumed that the cosmic spectrum is unchanged, so for an observed rate above $3,050 \mathrm{keV}$, a constant fraction of that rate is assumed between 2,450 and 2,800 keV and subtracted from the ROI integral. A similar exercise is conducted for the uranium series ROI, which was selected to extend from 1,720 to $2,400 \mathrm{keV}$; however, a portion of the ROI contribution is attributed to ${ }^{208} \mathrm{Tl}$ photons. This is accomplished by using a simulation of $2,615 \mathrm{keV}$ photons incident on a $2 \mathrm{in} . \times 4 \mathrm{in} . \times$ 4 in. $\mathrm{NaI}(\mathrm{Tl})$ crystal, applying an energy resolution function, and noting the ratio of energy depositions between $1,720-2,420 \mathrm{keV}$, relative to that above $2,650 \mathrm{keV}$, and subsequently multiplying the ${ }^{208} \mathrm{Tl}$ peak rate by this ratio to obtain the attribute photons in the ROI. The cosmic fraction is similarly subtracted, resulting in an estimated uranium series rate. For ${ }^{40} \mathrm{~K}$, the continuum surrounding the $1,460 \mathrm{keV}$ peak is fit as a second order polynomial, and the fraction of the peak ROI $(1,295-1,570 \mathrm{keV})$ from background is estimated by scaling (according to the fit background continuum polynomial) the integral in regions $1,150-1,295 \mathrm{keV}$ and $1,570-1,670 \mathrm{keV}$. Although these methods supply reasonable estimates at the true rates because of the low numbers of counts in a $1 \mathrm{~s}$ spectrum, they are strongly impacted by statistical uncertainties. The ${ }^{40} \mathrm{~K}$, uranium ROI, and ${ }^{208} \mathrm{Tl}$ median statistical error fractions for the $1 \mathrm{~s}$ intervals are $26 \%, 27 \%$, and $31 \%$, respectively, compared to $1 \%$ for gross counts. 

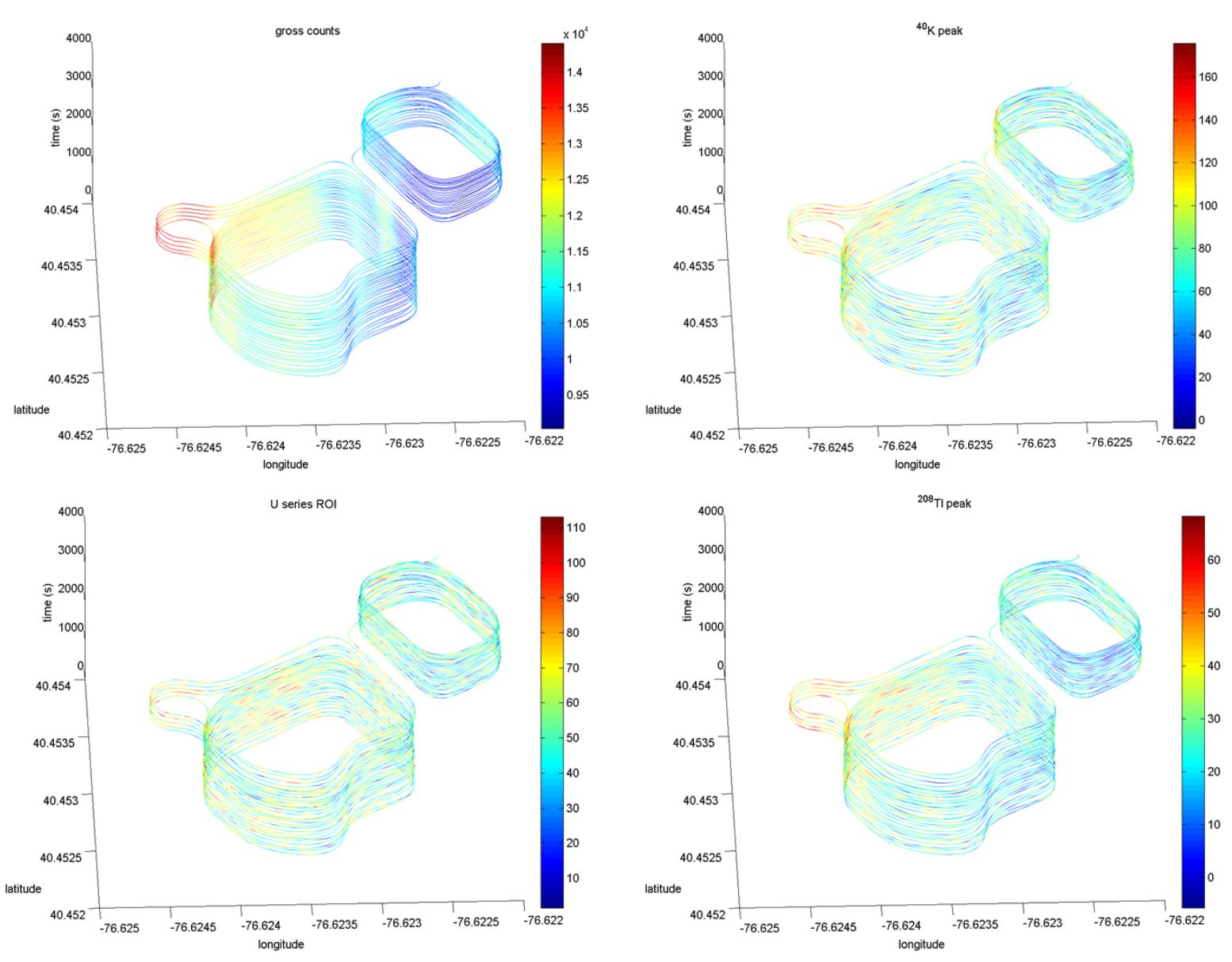

Figure 4.7.8. Time- and position-dependent $1 \mathrm{~s}$ count rates from RadMAP NaI(TI) array. (Top left) Gross counts, (Top right) ${ }^{40} \mathrm{~K}$ peak ROI, (Bottom left) uranium ROI 1,720-2,450 keV, and (Bottom right) ${ }^{208} \mathrm{Tl}$ peak ROI. For the ROI, events attributed to background were subtracted as described in the text.

From Figure 4.7.8, one can assert that the gross count rate observed at a particular location appears to stay consistent as a function of time. Because of counting statistics, it is less clear what can be asserted for the region of interest fits. This has motived an effort to more robustly decompose an observed background into physically relevant components, which is an ongoing effort. Instead, one may aggregate observed count rates spatially. This is accomplished by discretizing the measured region ( $3 \mathrm{~m} \times 3 \mathrm{~m}$, in this case), and each time the vehicle's GPS reports being within a grid point, the time duration within the grid is summed, the counts rates updated, and plotted (Figure 4.7.8). In order to increase counting statistics, only the bins with at least $10 \mathrm{~s}$ of data are collected. The duration of measurement within the grid and the aggregated rates for potassium, uranium, and thorium for measurement times $>10 \mathrm{~s}$ are shown in Figure 4.7.9. Here, the statistical error within a single spatial bin is no more than $10 \%$. A spatial aggregation of gross counts is also shown, but all grid points are shown because of the excellent statistics of the gross spectrum. From this perspective, the ${ }^{208} \mathrm{Tl}$ and uranium ROI rates appear to most closely mimic the gross count rate (although uranium appears to show slightly reduced rates on the west side of the survey area), whereas the ${ }^{40} \mathrm{~K}$ ROI show some deviations, particularly on the west corner of loop B and near the Church St. apartment building. 

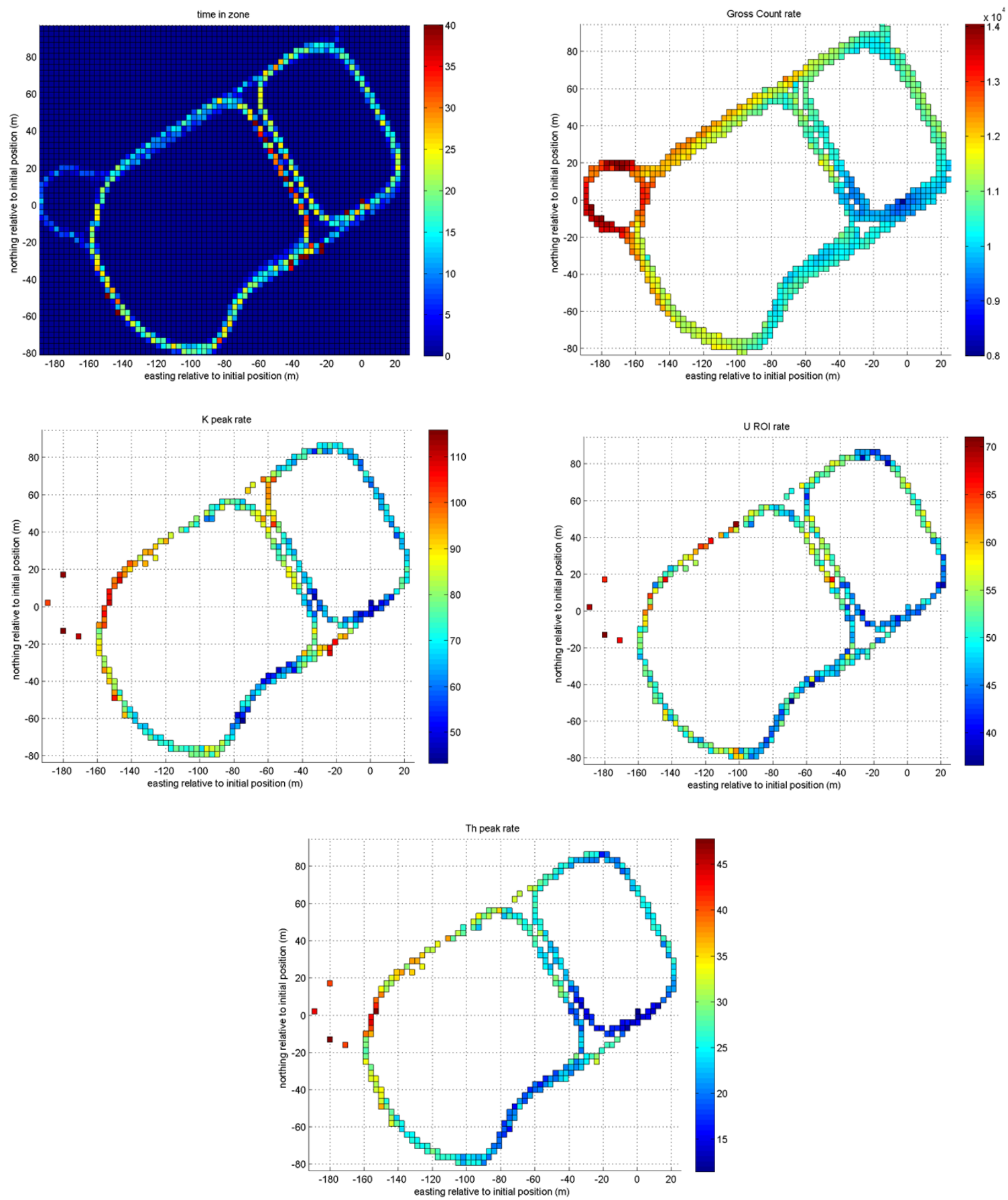

Figure 4.7.9. Spatially aggregated $\mathrm{NaI}(\mathrm{Tl})$ RadMAP array rates into a $3 \mathrm{~m}$ grid. The top left figure indicates the time spent in each grid point (and is cropped at $40 \mathrm{~s}$ ). Grid points with times greater than $10 \mathrm{~s}$ were considered for potassium (middle left), uranium (middle right) and thorium (bottom), but all times were considered for gross counts (top right).

Similar analyses were performed on the HPGe data, although far fewer counts were registered and better energy resolution provides better confidence that particular energy windows correspond to particular isotopes. As such, a cosmic continuum was fit and used as the only background subtraction for the ${ }^{208} \mathrm{Tl}$ peak, but uranium and potassium peaks were fit using a 3-window method. Four uranium-series ${ }^{214} \mathrm{Bi}$ 
peaks were integrated: 2,204, 2,448, 1,765, and $609 \mathrm{keV}$. All of these peaks are also found in airborne radon. Only 2,615 and 1,460 keV were integrated for ${ }^{208} \mathrm{Tl}$ and ${ }^{40} \mathrm{~K}$, respectively. The colorized temporalspatial rates for the gross counts $>70 \mathrm{keV}$ and the three components are shown in Figure 4.7.10. Mean rates are $1.0,3.2,4.6$, and $830 \mathrm{cps}$ for ${ }^{208} \mathrm{Tl}$, the four ${ }^{214} \mathrm{Bi},{ }^{40} \mathrm{~K}$, and gross counts, respectively. Clearly, statistical variability is a dominant feature in the three component spectra, whereas the gross counts plot is still fairly robust.
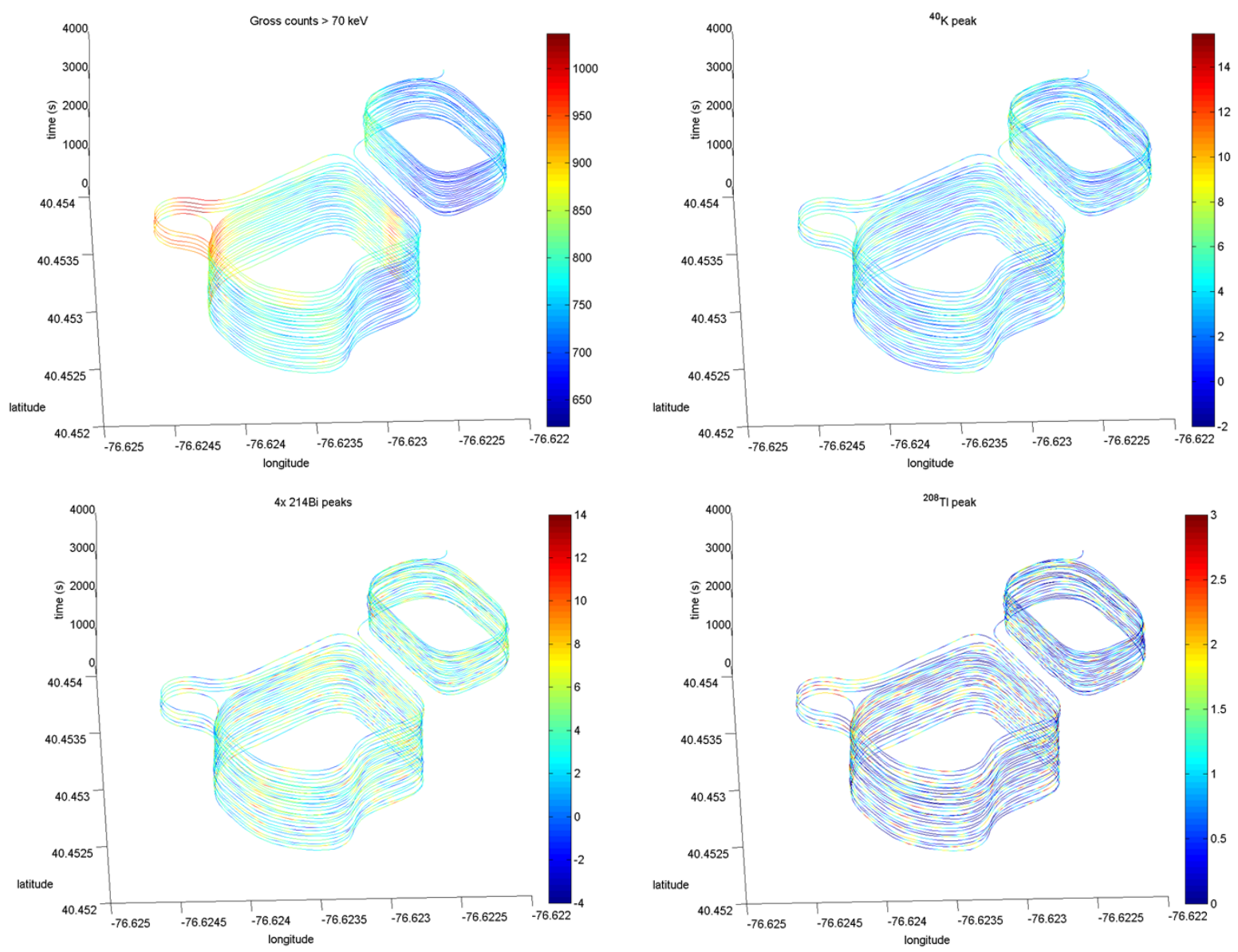

Figure 4.7.10. Time-and position-dependent $1 \mathrm{~s}$ count rates for the RadMAP IDM HPGe array. (Top left) Gross counts $>70 \mathrm{keV}$, (top right) ${ }^{40} \mathrm{~K}$ peak, (bottom left) four ${ }^{214} \mathrm{Bi}$ peaks due to uranium-series decays, and (bottom right) ${ }^{208} \mathrm{Tl}$ peak.

Spatial aggregations were conducted with the rates shown in Figure 4.7 .10 in a $3 \mathrm{~m}$ grid, analogously to the descriptions for the $\mathrm{NaI}(\mathrm{Tl})$ array. The resulting distributions are shown in Figure 4.7.11; however, the time-in-zone array is not shown again because it is identical to that shown in Figure 4.7.9. Statistical uncertainties in the spatial bins are less than $5 \%$ for gross counts but are $10 \%-25 \%$ for potassium, and worse for uranium and thorium. 

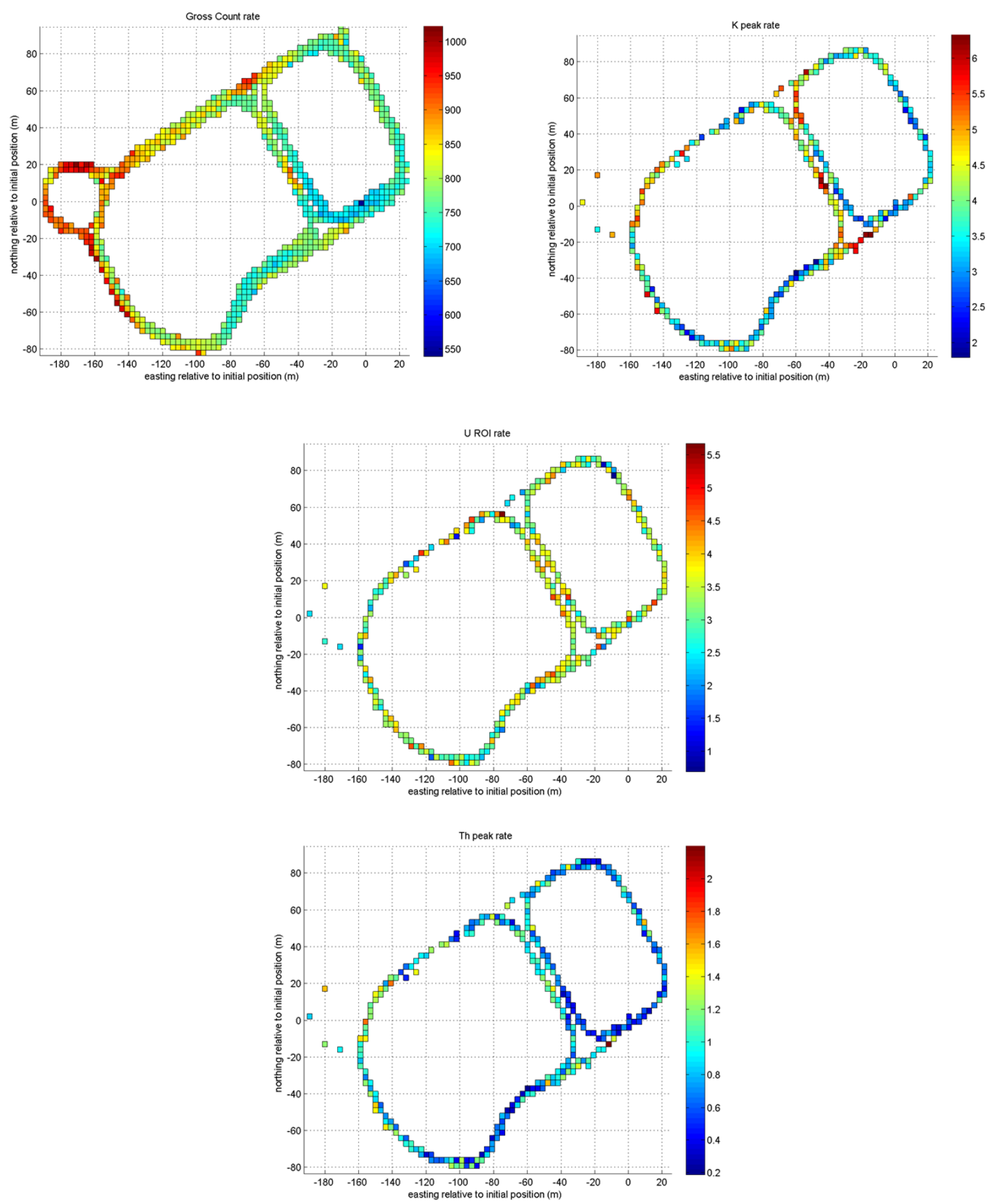

Figure 4.7.11. Spatially aggregated RadMAP IDM array rates into a $3 \mathrm{~m}$ grid. Only grid points with times greater than $10 \mathrm{~s}$ were considered for ${ }^{40} \mathrm{~K}$ (top right), four ${ }^{214} \mathrm{Bi}$ peak due to the uranium-series decays (bottom left) and ${ }^{208} \mathrm{Tl}$ due to thorium decay (bottom right). All times were considered for gross counts, which had a $70 \mathrm{keV}$ threshold applied (top left).

The graphics in Figure 4.7.11 continue to show similar trends to those shown for the NaI(Tl) array, particularly that most spatially aggregated rates mimic the gross count trends. The strongest exception is 
the potassium rate near the Church St. apartment building. However, the uranium data appear to poorly match the gross counts trend, which may be indicative of radon presence and because the $\mathrm{NaI}(\mathrm{Tl})$ analysis may still include some thorium series data in the.

The final analysis performed with respect to the behavior of the RadMAP NaI(T1) and IDM arrays was to directly consider the lap-to-lap consistency of the measured radiological signature. Given the limited counting statistics, gross counts will be the basis of this analysis. For each of the Main St. uphill loops, the distance along Main St. compared to the center of the northern intersection served as a reference. By interpolating GPS-reported positions along the Main St. dimension (which is called $x$ in this analysis), the approximate times corresponding to each $2 \mathrm{~m}$ interval along $x$ were identified and the list-mode $\mathrm{NaI}(\mathrm{Tl})$ and HPGe data were binned according to those times. Then for each pass, the count rate, $N / d t$, was calculated as a function of $x$. Here, $N$ is the number of gross counts during a $2 \mathrm{~m}$ interval, and $d t$ is the GPS-interpolated time interval during which the vehicle was within that $2 \mathrm{~m}$ interval. These values and their statistical errors are shown in Figure 4.7.12 for both the $\mathrm{NaI}(\mathrm{Tl})$ (left) and HPGe (right). Also shown as the heavy black line is the weighted mean rate versus $x$, where each contribution was weighted by $1 / \sigma^{2}$, where $\sigma(x)=\operatorname{sqrt}(N) / d t$. Also shown are histograms of the relative deviation between each observation and the weighted mean. These are expected to follow a Gaussian distribution, which is overlaid in red.
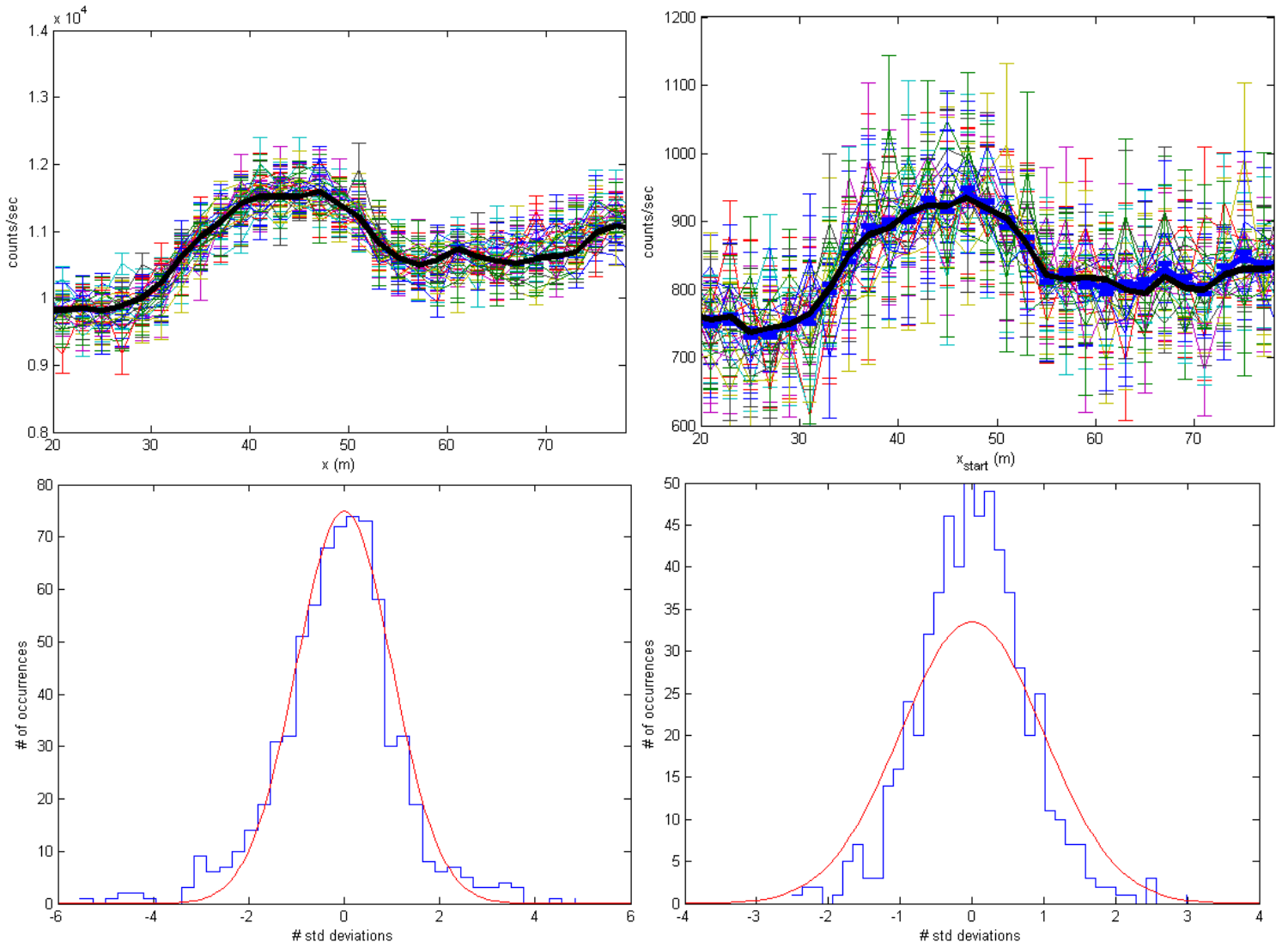

Figure 4.7.12. Comparison of RadMAP NaI(TI) and IDM array. (Left) RadMAP NaI(TI) and IDM (Right) array gross count rates for each uphill, east-side loop along Main St. binned into $2 \mathbf{m}$ bins along the dimension of Main St.Each loop is shown as a separate color with error bars, and the weighted mean rate is shown as the heavy black line. (Below) Histograms of the relative deviations from the weighted mean of each $\mathbf{2} \mathbf{m}$ measurement are shown. Also shown in red are the expected Gaussian distributions. 
From this analysis, the $\mathrm{NaI}(\mathrm{Tl})$ system appears to be providing consistent results for the vast majority of passes, although a handful of slight outliers were observed. Conversely, the HPGe array indicated less variability point-to-point than would be expected. This observed reduction in variability may be because the HPGe array is extended spatially along the length of the RadMAP trailer. This likely creates some correlation between adjacent observations that effectively reduce variability.

Overall, the gamma-ray detection systems functioned well during the data collection campaign providing good statistics at varying velocities, while also demonstrating stability for consecutive passes. The majority of the site demonstrated a level of spectroscopic homogeneity (in that spectra appear similar in shape but vary in magnitude), but there were a couple of regions that indicated heightened ${ }^{40} \mathrm{~K}$ rates, particularly the measurements near the apartment building on Church St.

\subsubsection{Dynamic Neutron Detector Measurements}

The EJ-309 liquid scintillator LS neutron detector array was constructed, installed, and calibrated in collaboration with Lawrence Berkeley National Laboratory and Sandia National Labs. Sandia continues to maintain the array and has led all previous analyses of data generated by the neutron array. However, for the purposes of this report, it is worthwhile to document the functionality of the detector array during the RadMAP campaign at the MOUT facility. Data generated by the LS array are saved as waveforms, digitized at $250 \mathrm{MHz}$ sampling frequency. The LS system operated throughout the campaign with the exception of the final hour of the Day 1 measurements and the final $100 \mathrm{~min}$. of Day 2 when the system went down unintentionally and was not brought back online until the following day. Gross count rates across Day 1 measurements for the 16 different detectors are shown in Figure 4.7.13.
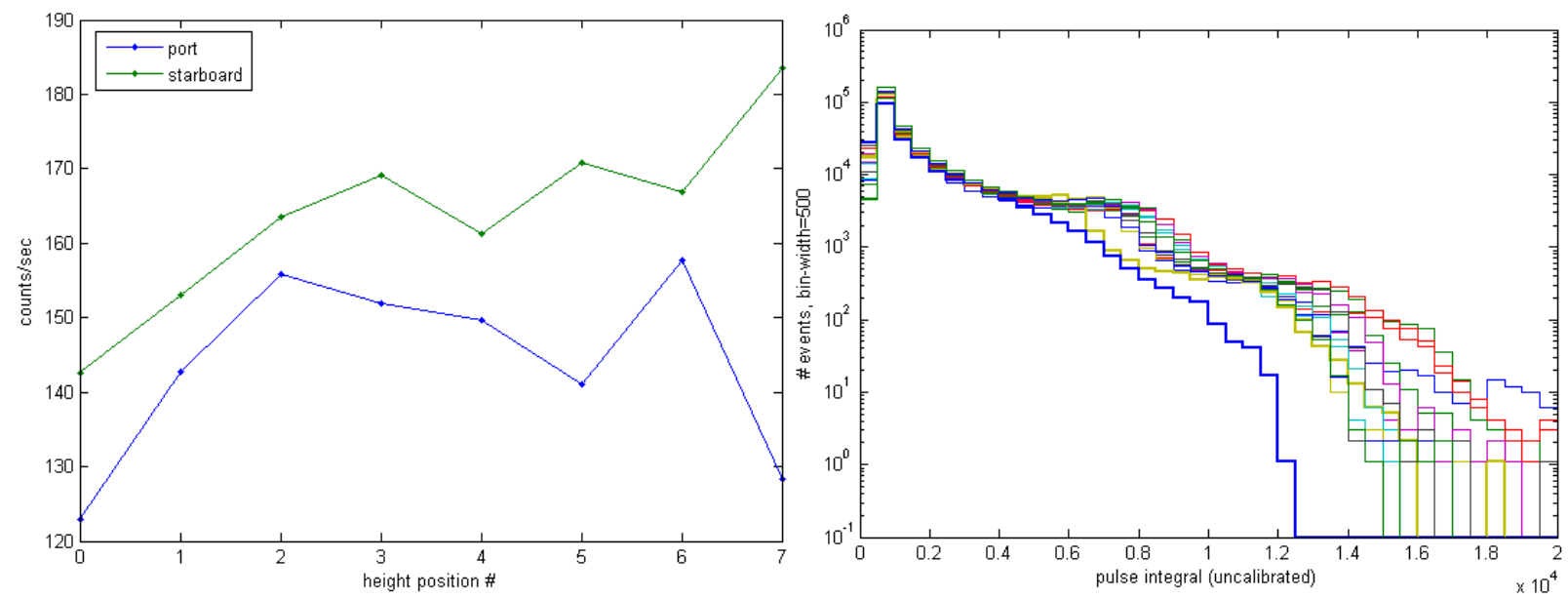

Figure 4.7.13. Liquid scintillator neutron detector Day 1 measurements. (Left) Gross LS count rates for 16 detectors. Height position 0 corresponds to the bottom of the array and 7 corresponds to the top. (Right)

Gross pulse integral spectra for the $\mathbf{1 6}$ detectors. The detector in the top height position on the port side (\#7) is demonstrating qualitatively different behavior from the other 15 detectors, including lower gain and worsened energy resolution.

For the entire set of LS events, two convolutional pulse integrations are performed, one with a short integration window (28 ns) and the latter with a longer window (400 ns). The ratio of the maximum values of these two integrals for each pulse is plotted against the long integral for all 16 detectors in the left side of Figure 4.7.14 for a $30 \mathrm{~min}$. interval during data collection activities on September 1. On the right plot of Figure 4.7.14, two digitized waveforms are presented. The waveforms have comparable 
widths, but the red wave form, which was categorized as the result of a gamma ray, has a larger fullheight, whereas the blue waveform, which was categorized as a neutron event, generally has a larger amplitude after sample number 15 as is more evident in the inset. The long integral and the long-short ratio are 3,075 and 1.35 for the indicated neutron waveform compared to 4,687 and 1.12 for the gammaray event.
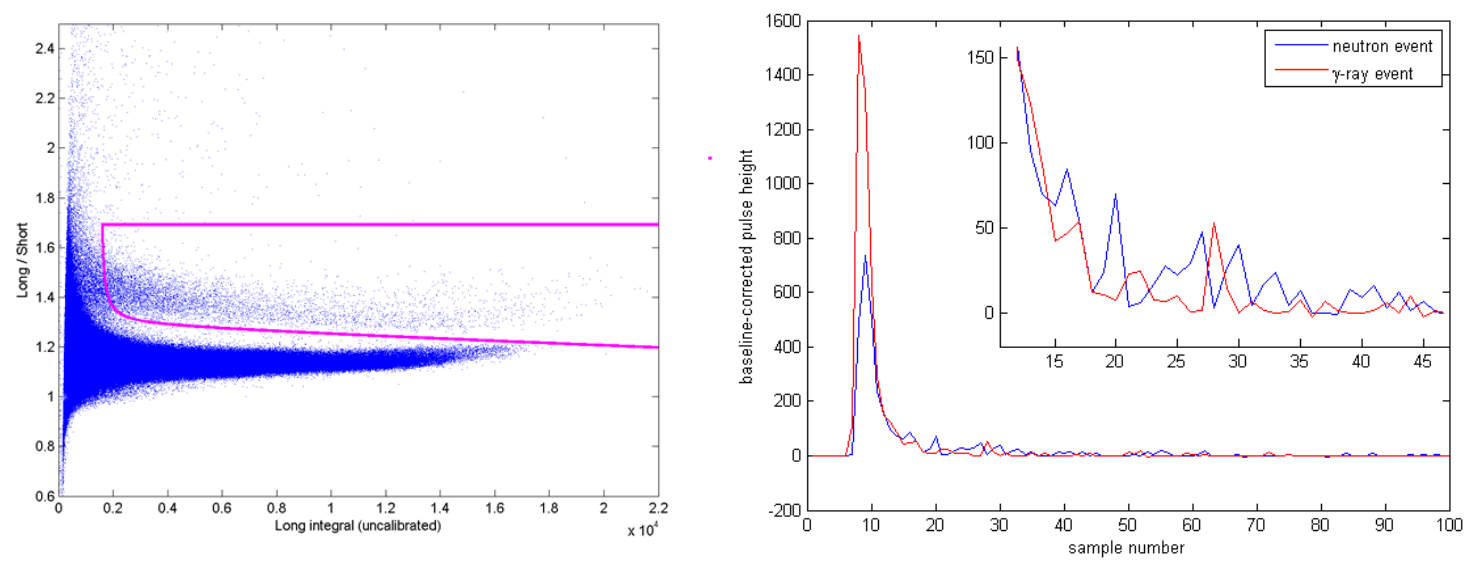

Figure 4.7.14. PSD plots for 28 and 400 ns pulse integrations across RadMAP 16 LS detectors. (Left) PSD plot of the ratio of the maximum $400 \mathrm{~ns}$ pulse integration to a $28 \mathrm{~ns}$ pulse integration vs the $400 \mathrm{~ns}$ integral for all

16 detectors during the first 15 laps during Day 1 . The region surrounded by magenta corresponds to the events that were attributed as neutrons. (Right) Two digitized events; blue waveform categorized as a neutron event and red categorized as gamma event.

The right section in Figure 4.7.15 indicates neutron count rates over a $30 \mathrm{~min}$. time period while the RadMAP system was conducting laps during Day 1. The time-bins in this plot are 20.09 wide. The neutron rate appears to vary in time, although disentangling that variation from inherent statistical variations is not trivial. It is also of interest to inspect the distribution of the $1 \mathrm{~s}$ neutron count rates, which are more relevant because of the truck velocity. The frequency of neutron count rates in $1 \mathrm{~s}$ intervals are shown as a histogram in the right plot of Figure 4.7.15. Also plotted is a Poisson distribution whose mean is the observed mean neutron count rate during the $30 \mathrm{~min}$. interval. The distributions are qualitatively similar, although the observed rates include significantly higher incidence of 0 neutron counts in a $1 \mathrm{~s}$ interval than would be anticipated by the Poisson distribution. 

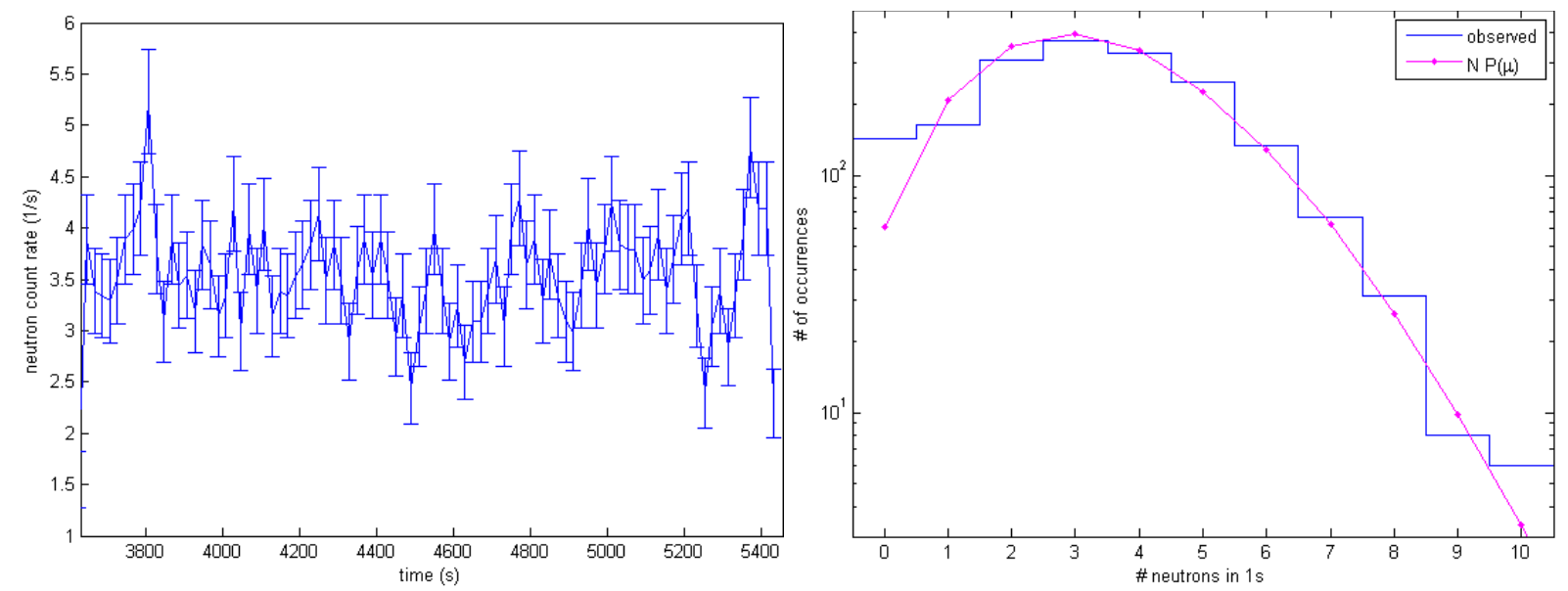

Figure 4.7.15. RadMAP neutron count rate as a function of time during Day 1. (Left) The summed neutron count rate across the 16 detector array binned in $20 \mathrm{~s}$ bins for times 60-90 min. into the Day 1 laps.(Right)A histogram of the observed neutron count rates during the same period binned into $1 \mathrm{~s}$ intervals. Also plotted in magenta is a Poisson distribution with the same mean, normalized by the number of observed $1 \mathrm{~s}$ intervals.

Given the statistical uncertainty evident in the observed rates, the neutron counts are spatially aggregated in an attempt to gain better insight as to whether there is statistically significant spatial variability in observed neutron count rates across the site. Similar to what was performed on the IDM and NaI(Tl) arrays, the data were aggregated spatially and only those bins in which at least $10 \mathrm{~s}$ of data were collected are subsequently considered. Given $10 \mathrm{~s}$ of data, the fractional error would still remain $17 \%$, which is indicative of the difficulty obtaining good statistics while operating a mobile neutron detector. Despite this, spatially aggregated neutron count rate for all Day 1 measurements is presented where data were obtained for at least $10 \mathrm{~s}$ in the left plot in Figure 4.7.16. There is very little to suggest statistically significant spatial variability in neutron rates across the site. The statistical deviation of these spatially aggregated count rates are also binned and plotted in the right plot of Figure 4.7.16. As expected, much of the data appear to follow a Gaussian distribution, implying that the observed variability is due to statistics alone. However, two spatial points show appreciable deviation (i.e., one 7- $\sigma$ below, and another 4- $\sigma$ above the mean). These two points correspond to locations where the RadMAP parked for a substantial period (15 and $30 \mathrm{~min}$., respectively) and in attempt to increase counting statistics. The two locations are circled in red in the left image of Figure 4.7.16. Although their observed mean neutron rates fit well within the range observed at FTIG, they do demonstrate statistically appreciable variability from the mean. This indicates that neutron flux spatial variability may exist, but the dynamic measurements are unable to resolve them. 

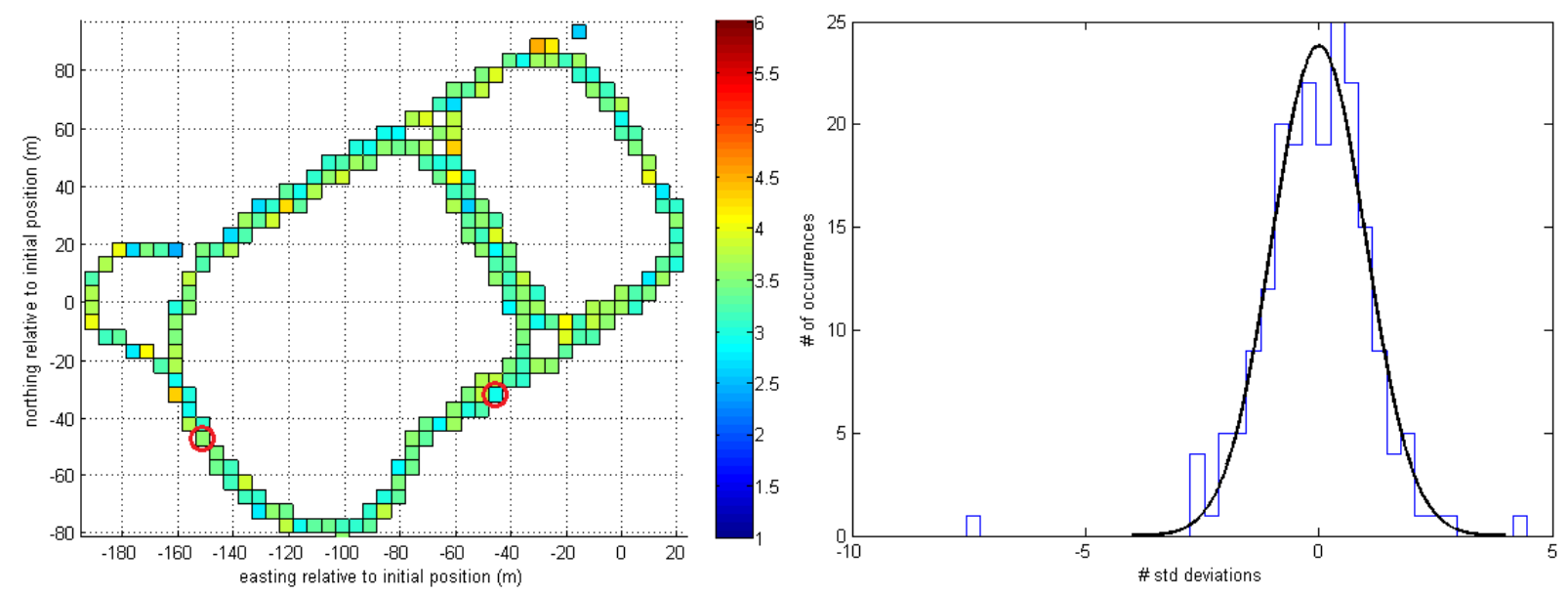

Figure 4.7.16. Dynamic RadMAP neutron measurements collected of FTIG. (Left) Spatially aggregated neutron count rates across the Day 1 measurements for pixels wherein greater than $10 \mathrm{~s}$ of data were collected.Two circled pixels correspond to the two locations that deviated statistically significantly from the mean because of the longer measurement intervals at these positions. (Right) Histogram of the deviation from the mean of the observed neutron count rate in each pixel in terms of number of standard deviations. Plotted in black is a Gaussian distribution that represents statistical variability.

\subsubsection{Static Measurements}

Static measurements were conducted primarily during Day 2, although some occurred on Days 1 and 3. Analyses were conducted to check that there were no radiological changes during each static measurement. This was accomplished by examining the gross count rates, as well as the potassium/uranium and thorium rates, as a function of time and comparing the observed variability to a null hypothesis that these rates should vary according to a Gaussian distribution with mean and variance of the observed average count rate. $\mathrm{NaI}(\mathrm{Tl})$ gross counts are specifically highlighted because it has the smallest fractional statistical error, implying that any changes would be most easily observed. Histograms of the deviation of observed gross count rates, normalized by standard deviation for the $\mathrm{NaI}(\mathrm{Tl})$ array, are plotted a long with a Gaussian distribution in the left plot of Figure 4.7.17. The observed rates follow a Gaussian trend with the exception of the data indicated in green. This run consisted of the field measurements that occurred between 6:39 a.m. and 7:01 a.m. PDT as indicated in Table 4.7.3. In this measurement, RadMAP remained in the same location but was parked adjacent a utility truck, which drove away from its position after approximately $10 \mathrm{~min}$. of measurement. Clearly, the truck had a significant impact on the measurement. The time-dependence of this particular measurement is shown as the right plot of Figure 4.7.17. The data included in this measurement was also separated into two static measurements whose behavior are also included in the left plot. 

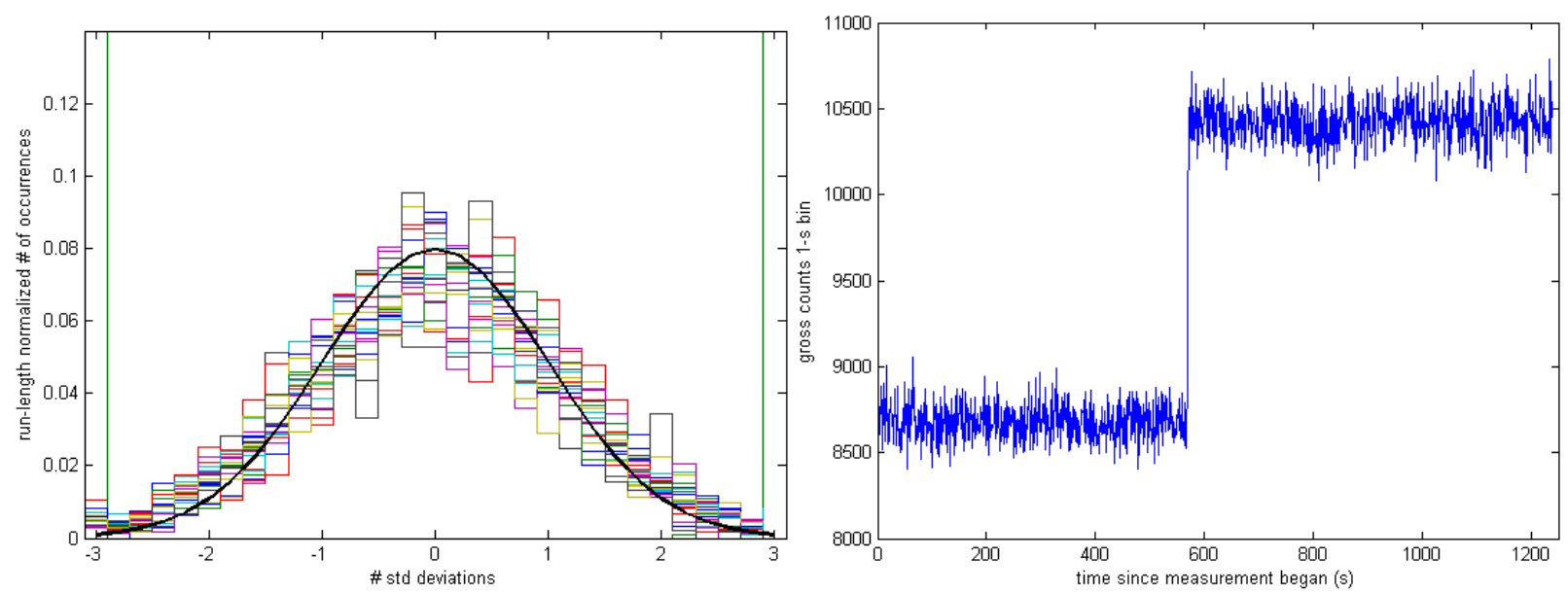

Figure 4.7.17. RadMAP static NaI(Tl) Day 2 measurements. (Left) A series of histograms of the deviation normalized $\mathrm{NaI}(\mathrm{Tl})$ gross count rates relative to the measurement mean for 20 Day 2 static measurements as well as the combination of the with- and without-truck measurements of the northern field (green).(Right) The time series of $\mathrm{NaI}(\mathrm{Tl})$ gross counts for the pair of with- and without-truck measurements. The truck was moved approximately $590 \mathrm{~s}$ into the measurement.

With the excellent statistics afforded by the static measurements, the count rates due to potassium, uranium, and thorium can directly be assessed by only integrating peak regions of interest in the HPGe data. The observed count rates in the HPGe data mirror the trend indicated in the left plot of Figure 4.7.17, but by integrating peaks, the HPGe gross counts as a function of static measurement position is obtained (Figure 4.7.18, top image) and potassium, uranium, and thorium rates as a fraction of the gross count rate. As discussed previously, 3-window ROI fitting was used for the $1,460 \mathrm{keV}$ peak for potassium and uranium was constructed as the sum of four peaks $(609,1,765,2,204$, and 2,448 keV) integrated using a 3-window ROI, and thallium used all events above $2,640 \mathrm{keV}$ that were not attributed to a power series fit of the cosmic continuum.

For the gross counts, certain phenomena are worth highlighting again. Of utmost interest is the fact that the lowest gross count rate was observed when RadMAP was parked adjacent the utility truck. When the truck left, and RadMAP was positioned overlooking a field, the count rate returned to within the site's normal rate. The lowest gross count rate measurements also correspond to the locations indicating the lowest potassium and thorium total rates, which can be explained by the fact that there are fewer objects within the near field resulting in both lower count rates and a higher fraction of observed signal being due to down-scattered radiation from the far field. The contrapositive, particularly observations along the west side of Main St. appear to hold as well. Notably, potassium and thorium fractions follow one another in many locations, but there are exceptions, particularly along Church St. and near the police station (measured on North St.). These observations indicate that although correlating gross counts to observed fractions of subtended solid angle could provide some insight (Section 4.7.6), by necessity there will be some additional insight that can only be obtained by examining particular spectral phenomena.

The neutron rates, derived via PSD of the data generated by Sandia's LS detector array was also examined to ascertain whether there were location-specific variabilities that could be observed despite the Poisson variability. The mean rates for each of the first 31 Day 2 static measurement locations, along with the statistical error caused by counting statics, are plotted in Figure 4.7.19. Thereafter, the neutron system exhibited anomalous behavior before going offline for the remainder of the day. The figure suggests that some appreciable neutron flux variability may be present across the site. A widely known cause of energetic neutron count rate variability is atmospheric pressure variations, which are examined in the remainder of this section. 

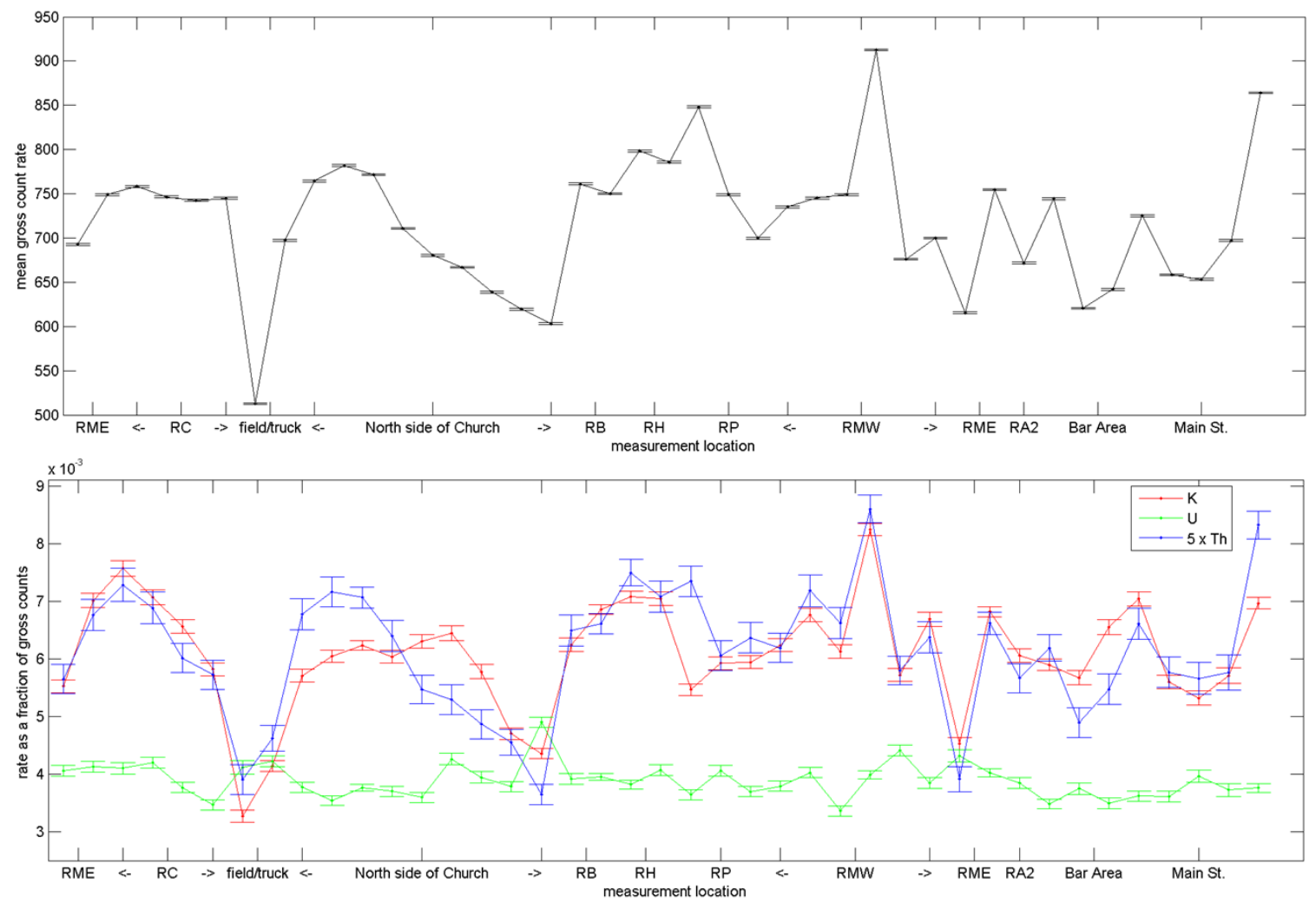

Figure 4.7.18. RadMAP integrated Day 2 gross counts from HPGe IDM array. (Top) IDM array gross counts as a function of Day 2 static measurement location. Locations are indicated on the abscissa.(Bottom) IDM Fractional rates due to integrated peaks. For the Tl-208 (Th) peak, the rate was multiplied by five to facilitate comparisons.

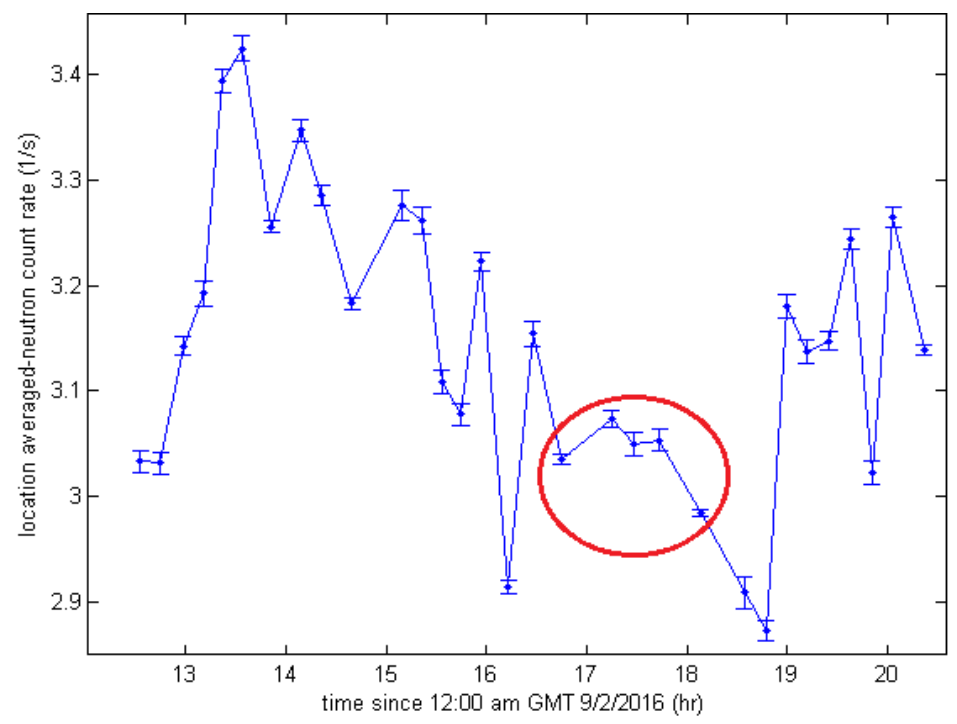

Figure 4.7.19. RadMAP derived static LS neutron count rate. The circled measurement times are shown in further detail in Figure 4.7.20 
Rather than averaging the observed neutron rate during the entirety of a static measurement, $250 \mathrm{~s}$ running averages are used, where each independent point (i.e., $250 \mathrm{~s}$ apart) has a Poisson error of 0.1 $(1 / \mathrm{s})$. The rates are also only shown for the portion of each measurement that is entirely static (e.g., the first and last $250 \mathrm{~s}$ of the running average is truncated for each static measurement). The running average neutron count rates are plotted for measurement locations 18-22 in the top of Figure 4.7.20, which correspond to the bar (RB), hotel (RH) and police station (RP) measurement locations in Figure 4.7.18 and to the circled points in Figure 4.7.19. These static locations were selected for inspection because measurements 18 and 22 were the longest-duration static measurements on Day 2. This facilitates observation of trends, particularly, upon application of the running average and truncation. From this analysis, the neutron count rate is observed to vary with time at some static locations and that the variation is both larger than expected from statistical considerations and does not appear to correlate with pressure. These observations cast doubt about the stability of the neutron detection system.

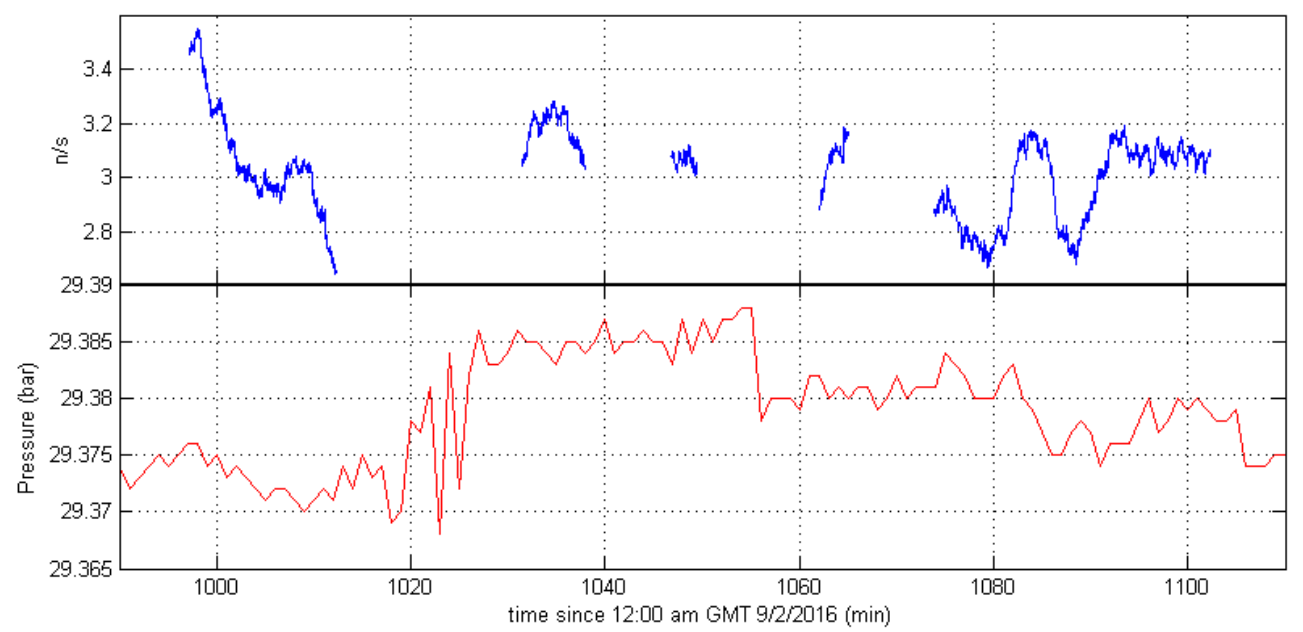

Figure 4.7.20. RadMAP Day 2 LS array static measurements. The statistical error associated with each independent data point (e.g., ones separated by 250 s) ranges between 0.10 and 0.12 . (Below) Observed pressure readings during the same measurement interval.

\subsubsection{RadMAP Weather Data}

During the measurement campaign, RadMAP fielded a Davis Vantage Vue 6250 weather station that collected temperature, barometric pressure, humidity, and rainfall data at $1 \mathrm{~min}$. frequencies. The weather system operated continuously during the entirety of September 1 into September 2 and was promptly shutdown after the collection activities on September 2 before being restarted on September 3 . The observed temperature, dew point, and pressure are shown in Figure 4.7.21 for the entirety of the campaign. Diurnal temperature variations are observed as are substantial swings in pressure. However, the pressure variability is foremost a result of the site's elevation of $760 \mathrm{ft}$, whereas the location where RadMAP was parked each evening was at $450 \mathrm{ft}$. A $1 \%$ pressure change every $300 \mathrm{ft}$ is expected. During single-day measurements, pressure changes of $\sim 0.15 \%$ were observed. Trends observed on-site by the RadMAP weather station closely resemble those observed by the stationary systems. It is also worth noting that the RadMAP system was not static during the entire measurement campaign. 


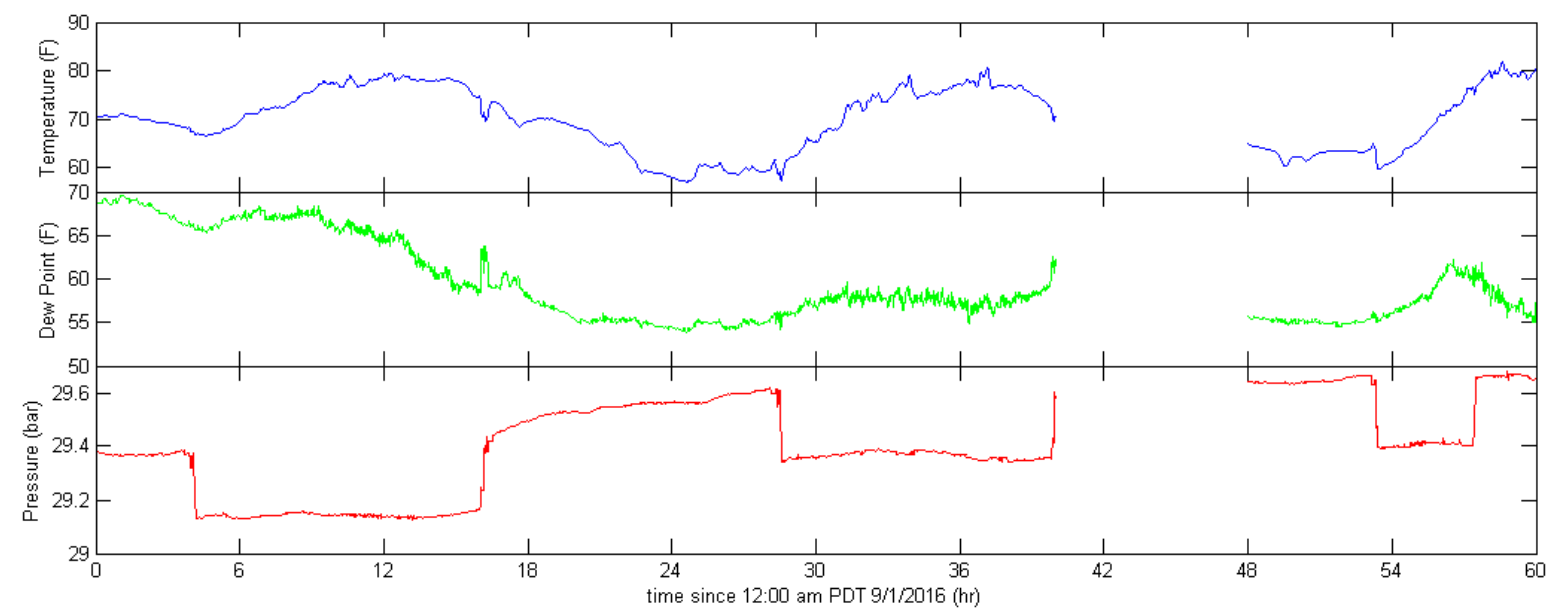

Figure 4.7.21. RadMAP MUSE 1 observed weather data.

\subsubsection{RadMAP Anomaly Algorithms}

RadMAP continuously operates two anomaly algorithms on $2 \mathrm{~s}$ spectra generated by both the $\mathrm{NaI}(\mathrm{Tl})$ array and the HPGe array. The algorithms and its characterizations are described in Ref. [12]. To summarize, the 3-window algorithm uses an energy range above and below selected peaks of interest to infer the most probable rate within the peak energy window. The inference was trained on a large set of background data collected within the San Francisco Bay Area. Observed peak ROI rates are divided by the FWHM that was observed during the training. This then produces an anomaly metric that roughly compares how well each 3-window range compares to the training set. The proprietary Poisson clutter split (PCS) algorithm is also trained using the same background dataset and produces an anomaly metric that is intended to correspond to the likelihood that an observed spectrum contains the indicated isotope compared with the likelihood it does not contain the isotope. PCS differs from the 3-window algorithm in that it also uses full-spectral analysis and has a description of the variability that was encountered during training encoded within its background. The algorithm's output detection metrics for the isotopes are listed in Table 4.7.5. 
Table 4.7.5. RadMAP algorithm anomaly list by isotope.

\begin{tabular}{|c|c|c|c|c|}
\hline \multicolumn{2}{|c|}{ 3-window algorithm isotope list } & \multicolumn{3}{|c|}{ PCS algorithm isotope list } \\
\hline 1. Positrons & $13 .{ }^{60} \mathrm{Co}(1332 \mathrm{keV})$ & 1. ${ }^{201} \mathrm{Tl}$ & $12 .{ }^{40} \mathrm{~K}$ & $23 .{ }^{232} \mathrm{Th}$ \\
\hline 2. ${ }^{99 \mathrm{~m}} \mathrm{Tc}$ & 14. ${ }^{137} \mathrm{Cs}$ & 2. ${ }^{60} \mathrm{Co}$ & 13. ${ }^{133} \mathrm{Ba}$ & 24. Depleted uranium \\
\hline 3. ${ }^{131} \mathrm{I}$ & $15 .{ }^{238} \mathrm{U}$ & 3. ${ }^{57} \mathrm{Co}$ & 14. ${ }^{240} \mathrm{Pu}$ & 25. ${ }^{237} \mathrm{~Np}$ \\
\hline 4. ${ }^{67} \mathrm{Ga}$ & 16. ${ }^{235} \mathrm{U}$ & 4. ${ }^{233} \mathrm{U}$ & 15. ${ }^{131} \mathrm{I}$ & 26. ${ }^{241} \mathrm{Am}$ \\
\hline 5. ${ }^{201} \mathrm{Tl}$ & 17. Neutrons_H & 5. ${ }^{54} \mathrm{Mn}$ & 16. ${ }^{228} \mathrm{Th}$ & 27. LEU \\
\hline 6. ${ }^{192} \operatorname{Ir}^{*}$ & 18. Neutrons_Fe & 6. ${ }^{67} \mathrm{Ga}$ & $17 .{ }^{235} \mathrm{U}$ & $28 .{ }^{22} \mathrm{Na}$ \\
\hline 7. ${ }^{241} \mathrm{Am}$ & 19. ${ }^{7} \mathrm{Be}$ & 7. ${ }^{68} \mathrm{Ge}$ & 18. ${ }^{238} \mathrm{U}$ & \\
\hline 8. ${ }^{133} \mathrm{Ba}$ & $20 .{ }^{40} \mathrm{~K}$ & 8. ${ }^{137} \mathrm{Cs}$ & 19. ${ }^{133} \mathrm{Sn}$ & \\
\hline 10. ${ }^{57} \mathrm{Co}$ & 21. ${ }^{214} \mathrm{Bi}$ & 9. ${ }^{109} \mathrm{Cd}$ & 20. ${ }^{226} \mathrm{Ra}$ & \\
\hline $11 .{ }^{60} \mathrm{Co}$ & 22. ${ }^{208} \mathrm{Tl}$ & 10. ${ }^{99 \mathrm{~m}} \mathrm{Tc}$ & 21. ${ }^{90} \mathrm{Sr}$ & \\
\hline 12. ${ }^{60} \mathrm{Co}(11$ & & $11 .{ }^{88} \mathrm{Y}$ & 22. ${ }^{239} \mathrm{Pu}$ & \\
\hline
\end{tabular}

Time-series of the 3-window algorithm alarm scores are shown for Day 2 in Figure 4.7.22. Near $t=40 \mathrm{~h}$, RadMAP was returning to the hotel when it passed a ${ }^{137} \mathrm{Cs}$ source that was within regulatory control and was packaged in U.S. Department of Transportation compliant container for shipping. That source registered a substantial ${ }^{137} \mathrm{Cs}$ anomaly score and RadMAP circled to stop in front of the source to acquire further data. The time series for that encounter are expanded in the right image of Figure 4.7.22.

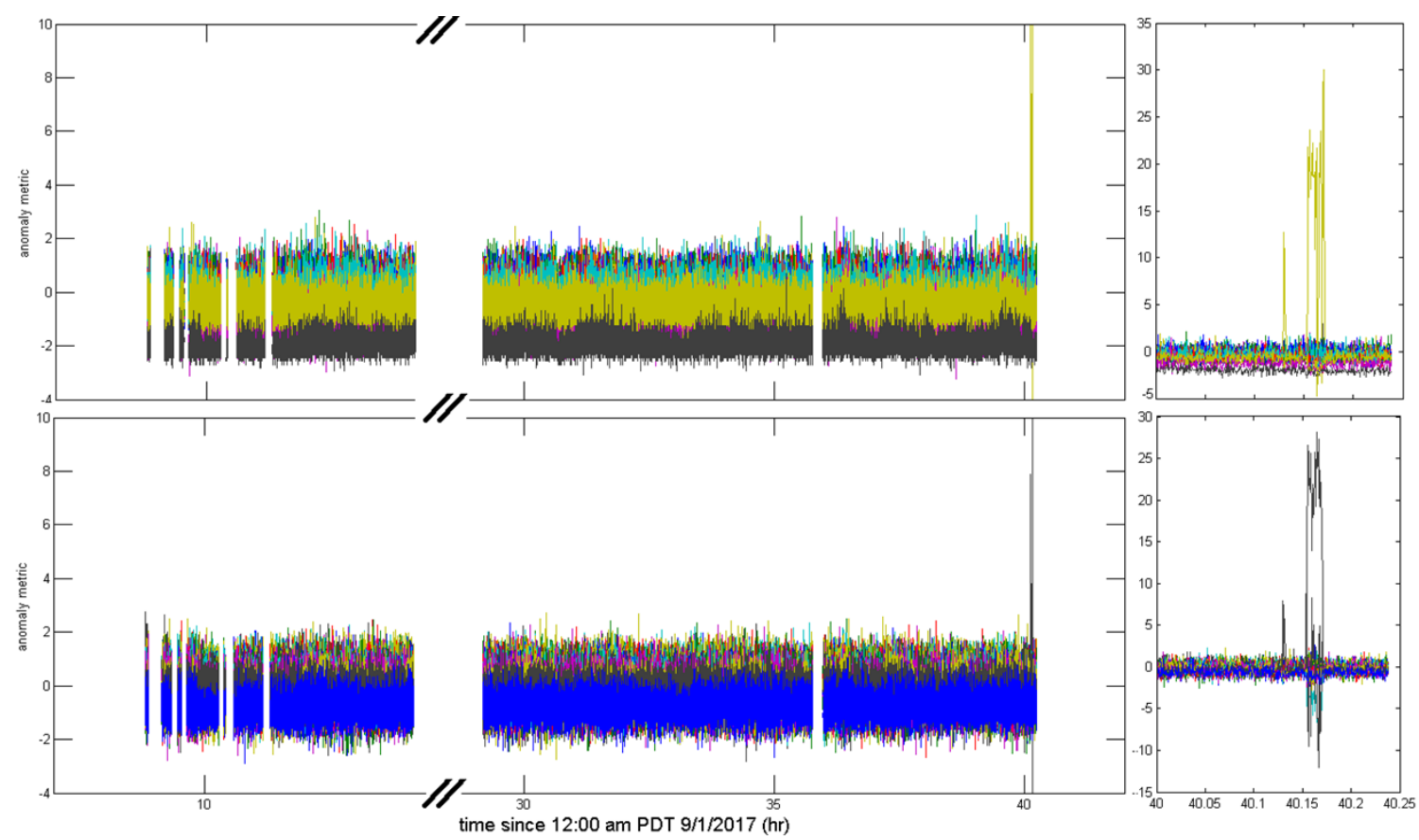

Figure 4.7.22. RadMAP time-dependent anomaly measurements for both HPGe and NaI(TI) arrays. Timedependent anomaly metrics for (above) 21 isotopes as measured by the HPGe 3-window anomaly algorithm and (below) 22 isotopes as measured by the $\mathrm{NaI}(\mathrm{Tl}) 3$-window anomaly algorithm. 
The spatial dependence of the ${ }^{137} \mathrm{Cs}$ encounter is shown in the left side of the plot in Figure 4.7.23. RadMAP's approaches close to the source produced large increases in anomaly scores. Each ${ }^{137} \mathrm{Cs}$ anomaly score for the indicated set was also plotted versus nominal distance from the source in the right plot. In general, scores generated during measurements with distance above $20 \mathrm{~m}$ were not larger than the baseline, whereas many scores below $10 \mathrm{~m}$ indicate substantial score increases. The initial pass-by when RadMAP drove approximately $13 \mathrm{~m}$ from the source did not result in an appreciable alarm metric change. Instead, it was the PCS alarm metric that prompted further investigation. The NaI(Tl) array also appeared to produce more reliable anomaly scores than the algorithm operating on the HPGe array, likely due to insufficient statistics within the peak regions.
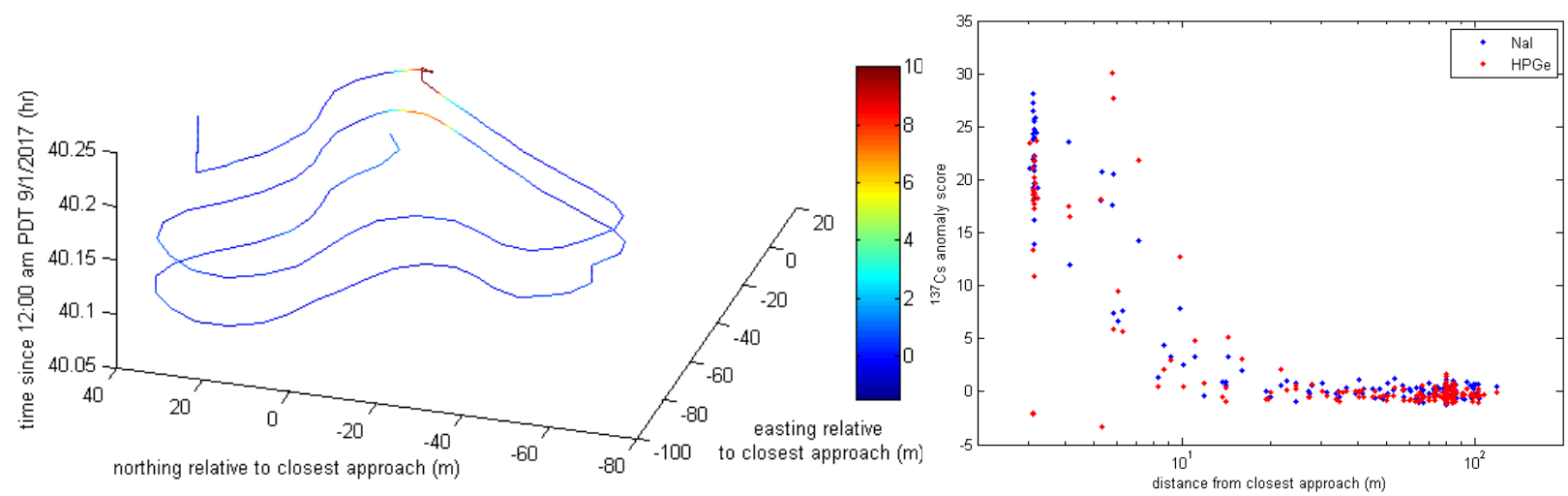

Figure 4.7.23. RadMAP 3-D map of anomaly measurement with algorithm score. (Left) 3-D plot of RadMAP location versus time, colorized by ${ }^{137} \mathrm{Cs} \mathrm{NaI}(\mathrm{Tl}) 3$-window algorithm anomaly score.Note, the color map was limited to 10, instead of the maximum observed score of 26. (Right) $\mathrm{HPGe}$ (red) and $\mathrm{NaI}(\mathrm{Tl})$ (blue) ${ }^{137} \mathrm{Cs} 3-$ window algorithm anomaly scores vs distance relative to the approximate location of the ${ }^{137} \mathrm{Cs}$ source.

Excluding the time of the encounter (after $t=40.1 \mathrm{~h}$ ), the NaI(Tl) 3-window algorithms output scores exhibited very little time-dependent variability except statistical variations. Scores from the $\mathrm{NaI}(\mathrm{Tl})$ array were quite Gaussian in behavior and the HPGe scores resembled mean-shifted Poisson distributions, although both systems demonstrated mean shifts away from zero because of spectral differences between the Pennsylvania region and the San Francisco Bay Area. The mean scores and standard deviations of the isotope anomaly scores are shown in the left plot in Figure 4.7.24. Whereas the 3-window algorithm metric values vary with a standard deviation near 0.5 , the PCS algorithm metrics depend on the resolution to which the algorithm is sensitive and can be substantially lower. The PCS mean values for the 28 PCS isotopes during the $2.5 \mathrm{~h}$ portion of the Day 2 measurement preceding the ${ }^{137} \mathrm{Cs}$ encounter are shown in the right plot in Figure 4.7.24 as solid lines, and the associated standard deviations are shown as dashed lines. For ${ }^{137} \mathrm{Cs} \mathrm{NaI}$, the mean value was $4.4 \mathrm{e}-3$ with a standard deviation of $1.6 \mathrm{e}-2$, which caused anomaly metric values near 1 to appear very significant. 

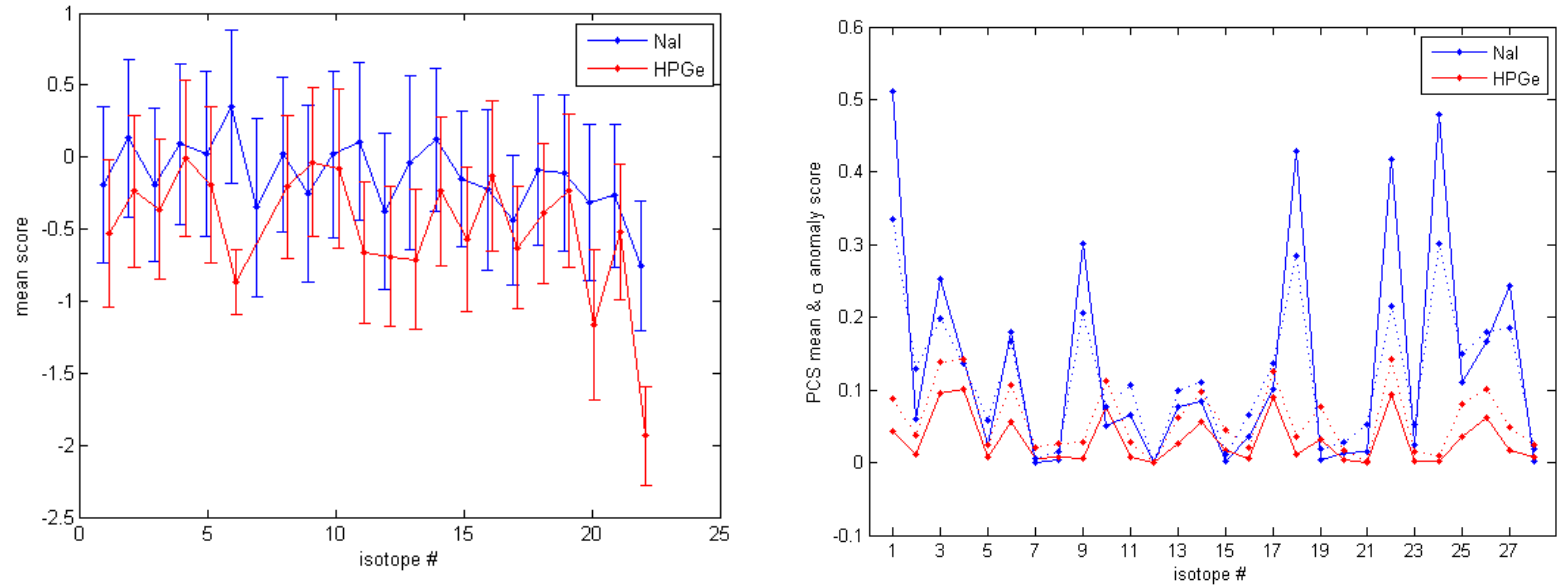

Figure 4.7.24. RadMAP scoring metric values for 3-window and PCS algorithms. Mean and standard deviation anomaly metric values for the 3-window algorithm (left) and for the PCS algorithm (right) plotted as a function of the isotope index given in Table 4.7.5.The dashed lines in the right plot correspond to the standard deviations of the values. For the 3-window algorithm, all Day 1 and Day 2 data were used except for the ${ }^{137}$ Cs encounters. For the PCS algorithm, only the $4 \mathrm{~h}$ preceding the ${ }^{137}$ Cs encounter are shown.

The significance of the ${ }^{137} \mathrm{Cs}$ PCS alarm metric is what enabled the RadMAP operators to observe the source. In the left plot in Figure 4.7.25, the time- and spatial-dependent PCS NaI(Tl) ${ }^{137} \mathrm{Cs}$ scores are shown. The initial pass-by the source was substantial enough to trigger a response. In the right plot, the PCS scores versus standoff are shown. The $\mathrm{NaI}(\mathrm{Tl})$ score that was observed at a standoff near $13 \mathrm{~m}$ triggered further investigation.
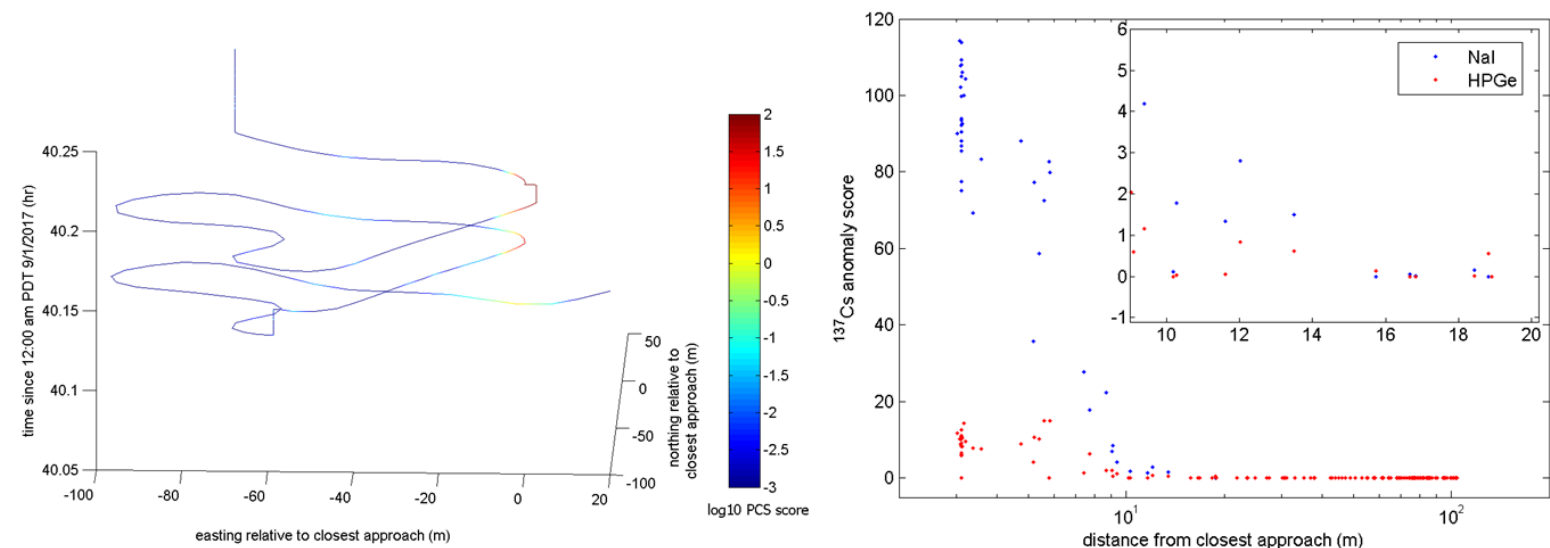

Figure 4.7.25. RadMAP path and algorithm metric during ${ }^{137} \mathrm{Cs}$ anomaly. (Left) Logarithms of NaI(TI) 137Cs PCS anomaly metrics plotted against time and position.(Right) ${ }^{137} \mathrm{Cs}$ PCS anomaly metrics for both the $\mathrm{NaI}(\mathrm{TI})$ and HPGe arrays versus approximate standoff distance from the source. Additional NaI(Tl) points are not visible above $\sim 20 \mathrm{~m}$ because they are covered by the corresponding point for the HPGe array.

Despite the fact that the PCS $\mathrm{NaI}(\mathrm{Tl})$ anomaly metric proved most sensitive in finding this ${ }^{137} \mathrm{Cs}$ source, the experience in Ref. [12] indicates that the HPGe array should be nearly as sensitive. One likely reason that this was not observed here is that both the background environment and the performance of the HPGe array differed substantially from the training sets. This is expected to be particularly true for the HPGe 
array, which was trained using data that was collected by the set of HPGe detectors that were previously fielded by RadMAP. The PCS performance is indicative of how advanced algorithms can benefit from substantial training data, but even without the availability of such data, their performance can be better than simpler algorithms.

\subsubsection{RadMAP Localization and Mapping}

The RadMAP system generates in real-time a GPS-based time series of system location outputs as well as IMU accelerometer outputs. In post-processing, RadMAP localization is derived using commercial software that combines GPS, IMU, and nearby reference station(s) to produce the inertial navigation system (INS) solution that has been considered to be most accurate. However, at FTIG, the nearest reference system was approximately 30 miles away, which is a larger offset than had ever been investigated with RadMAP, and the INS system was inadvertently set to write data at a lower frequency than was standard. This resulted in some difficulties getting the commercial software to provide solutions, and although this was resolved with assistance from the vendor, the quality of the solution may be worse than what had been observed in the San Francisco Bay Area RadMAP campaigns.

In post-processing, Lawrence Berkeley National Laboratory also operated a simultaneous localization and mapping algorithm (SLAM) that used the IMU and LIDAR data as inputs and generated a 3-D point cloud of the surveyed region while simultaneously deriving the positioning and orientation of the RadMAP system within the point cloud. Example point clouds are shown in Figure 4.7.26. Figure 4.7.27 also demonstrates synchronization between the LIDAR-derived point cloud and the video data by showing a single scan of LIDAR points in red, with color brightness indicating distance, overlaid on a stitched video still-frame. Clearly, the LIDAR does not cover as broad of a field of view (FOV) as the video; however, the LIDAR's coverage is sufficient to generate good representations of the scene near street level. Conversely, the tops of the buildings rendered in Figure 4.7.26 are mostly missing. 

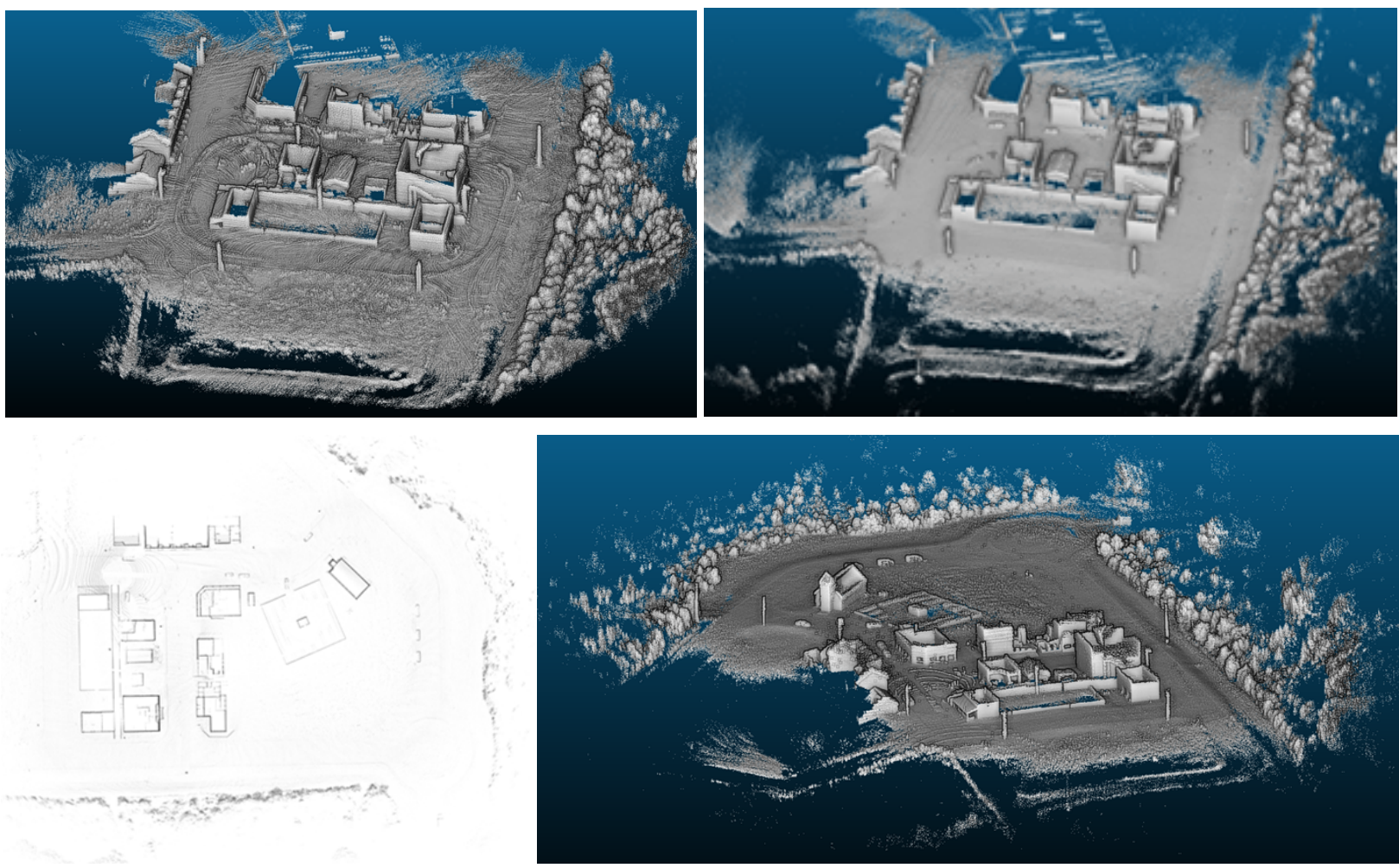

Figure 4.7.26. SLAM-derived point clouds of the FTIG CACTF facility. (Top left) Raw point cloud before SLAM self-consistency checks designed to removed transient features. (Top right) Same dataset after transient removal. (Bottom left) Top-down view of CACTF facility generated from $10 \mathrm{~min}$. of RadMAP collection. (Bottom right) Same dataset in perspective view.

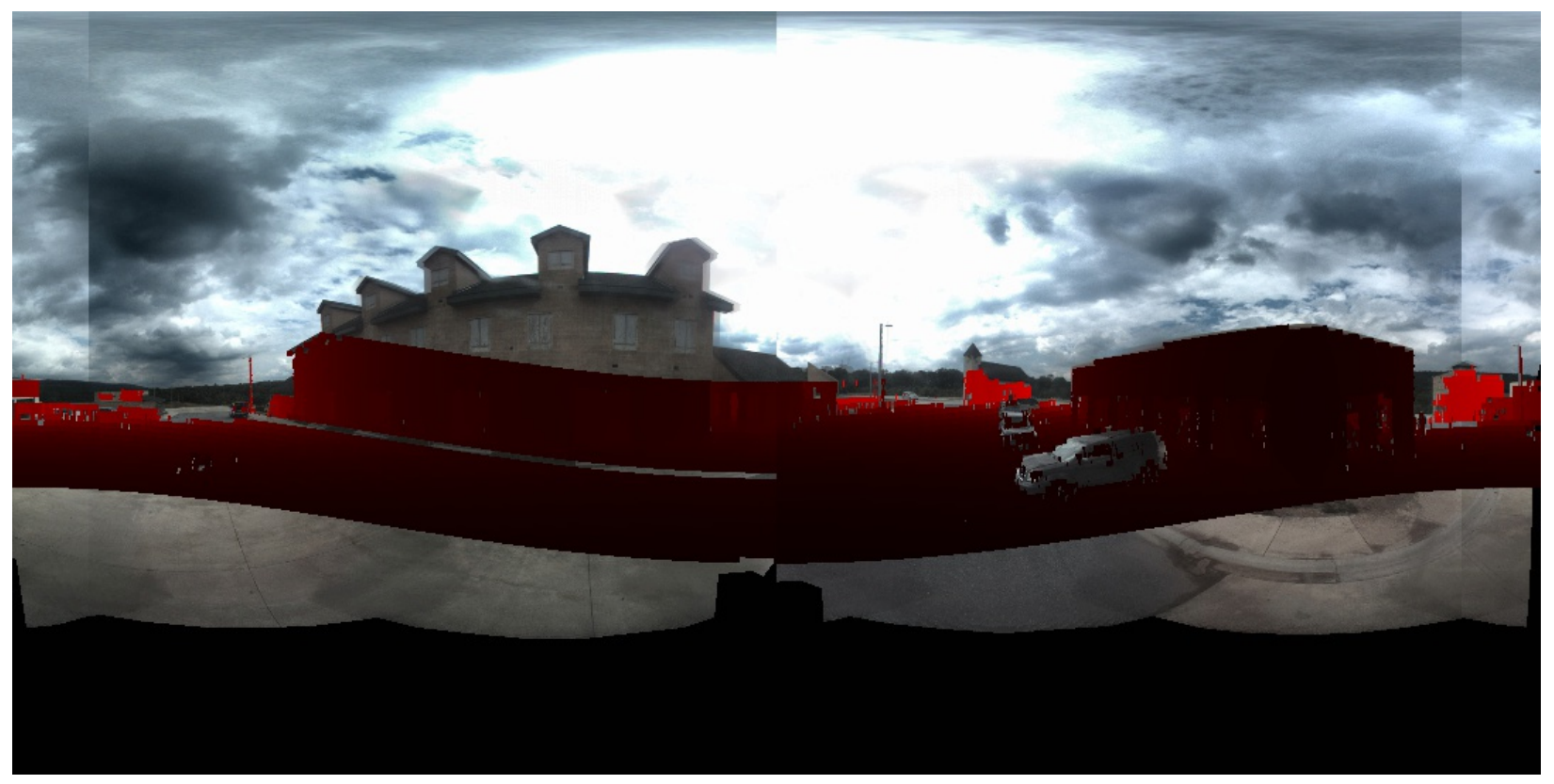

Figure 4.7.27. LIDAR values overlaid on RadMAP Ladybug $4 \pi$ still-frame image. 
Using the combination of accelerometers and LIDAR scans, the SLAM algorithm also provides odometry outputs, which may be compared to the localization information provided by the GPS and by the GPS/IMU. However, the datum in which the SLAM odometry is generated is only relative to the initial position. Therefore, the algorithm begins by determining the most probable initial position and heading of the system and subsequently updating the SLAM odometry map. This is accomplished by iterating across the initial position and heading of the SLAM odometry and selecting the position and rotation values that minimize the sum of the distances (on the horizontal plane) between each SLAM odometry point and the GPS or INS-based localization solution, interpolated in time to the SLAM time binning. This was achieved for an approximately 8 min. dataset comprising a Day 1 site survey. The best solution for the SLAM start position and rotation values differed slightly between comparisons to GPS and to the GPS/IMU solution. Those differences were $\Delta$ (easting) $=70 \mathrm{~cm}, \Delta$ (northing) $=40 \mathrm{~cm}$, and $\Delta(\theta)=0.175^{\circ}$. Minimizing the L1 distances instead of L2 distances showed an even smaller impact on optimal starting parameters. The left plot in Figure 4.7.28 shows the time-dependent horizontal distances between the best SLAM solutions for the INS and GPS and the corresponding solutions. The vertical black line near $160 \mathrm{~s}$ indicates that only times after that point were considered to minimize the residual because before then, the vehicle was not moving and producing stable, but possibly biased, GPS-based solutions. The right plot shows the time-dependent altitude profiles for the INS solution and for the best-fit SLAM solution. The same datasets are also plotted as a function of position and time in Figure 4.7.29.

Presumably, the SLAM solution is most precise when easily identifiable static features such as building corners are within the FOV of the LIDAR system, and it is least accurate when the LIDAR only has fields and trees within its FOV. This implies that the INS solution, which produces smaller residuals along Main St., does perform better than the GPS solution for the majority of the measurement, which is the anticipated result. However, the INS solution appears to initialize poorly, and both the GPS and INS solutions appear to be inaccurate during the stationary portion of this dataset. That the SLAM solution differs consistently from the INS solution in the northwest portion of the site which is to be expected because the LIDAR point cloud comprises the fewest quality landmarks for tracking.
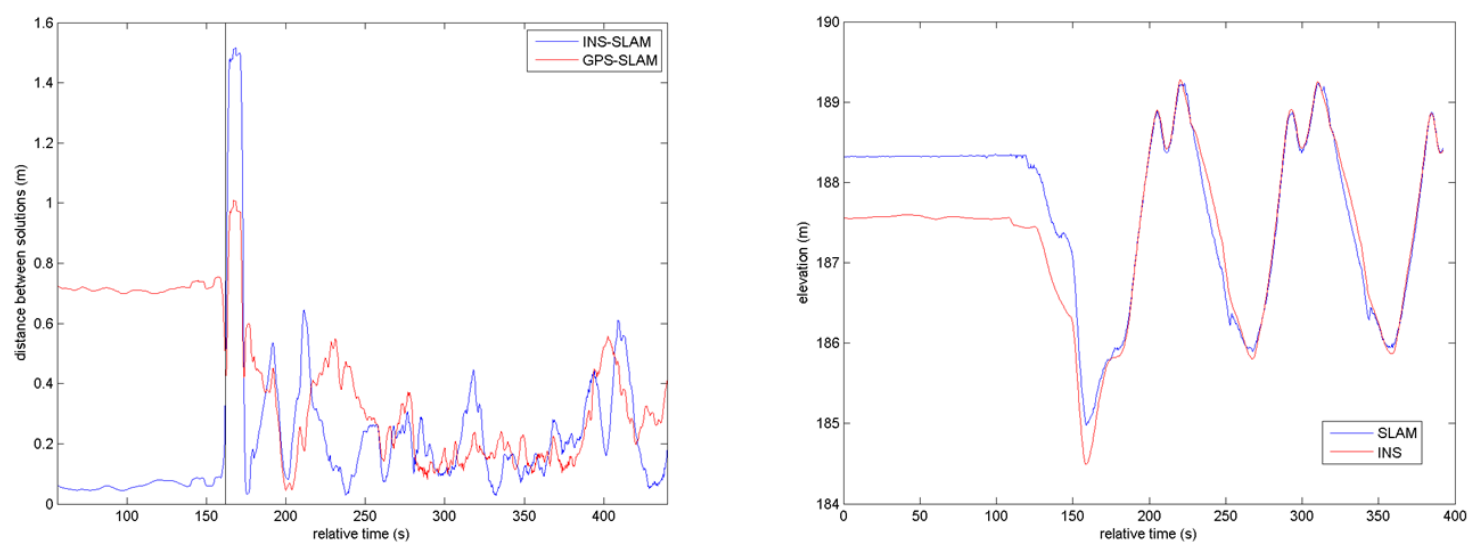

Figure 4.7.28. Analysis of RadMAP SLAM odometry algorithm. (Left) Horizontal distances between the INS and GPS solutions and the SLAM solutions that produce the minimal distance residuals after the time indicated by the black vertical line.(Right) Time-series elevations of the best SLAM solution and the INS solution. 


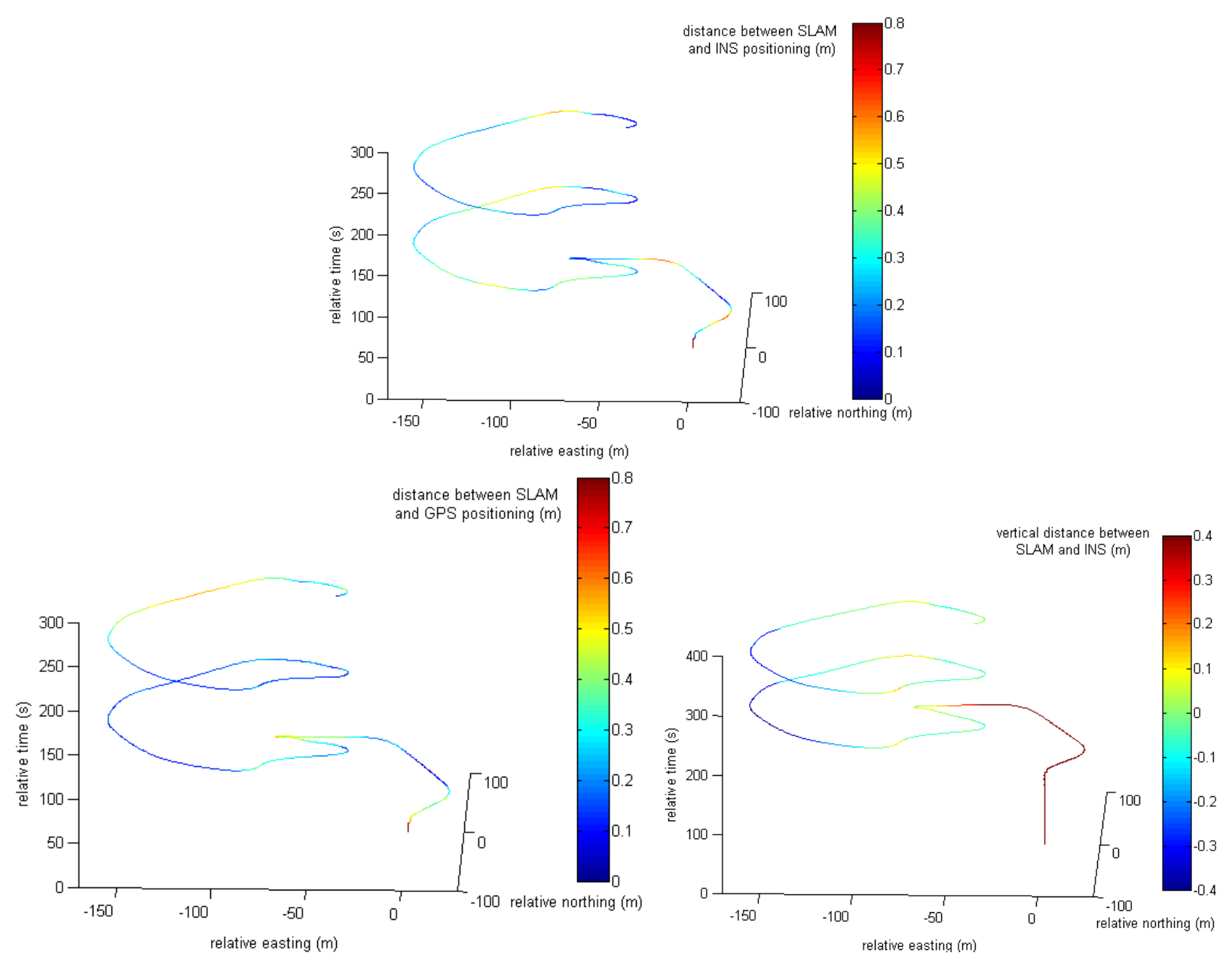

Figure 4.7.29. RadMAP path during SLAM algorithm measurements. Note, the maximum values of the color axes have been limited to highlight other features.

\subsubsection{RadMAP-RSI Comparison}

During a portion of the Day 1 laps (and again on Day 3), RSL trailed RadMAP in a vehicle that was configured in a manner typically used for radiological search and survey missions. The RSL system consisted of a Radiation Sensing Inc. (RSI) NaI(Tl)-based radiation detection system that is read-out at $1 \mathrm{~Hz}$ and a GPS unit located in the trunk of an SUV. The RSI readout generates spectra without performing coincident summing across the individual detector modules. The RadMAP spectra were similarly generated, although the list-mode data also enables different methods of analysis. The left plot in Figure 4.7.30 compares the spectra generated by the RSI system to those generated from the RadMAP $\mathrm{NaI}(\mathrm{Tl})$ array data during the same period. The RSI system has appreciably better resolution, which is most likely because the RadMAP NaI(Tl) crystals are older and less protected from the environment, which over the years can result in hydroscopic degradation of the crystal surfaces, reducing light output, and worsening resolution. The RadMAP system generated 7.4 times as many events during the concurrent measurements, so the RSI spectrum shown is scaled by 7.4. It is also evident that a larger fraction of the RSI events are low-energy. If a threshold of $400 \mathrm{keV}$ is applied, the RadMAP system generated 11.3 times as many events as the RSI system. The RSI system spectrum, scaled by 11.3 , is also shown in the plot. With this scaling, the spectral similarity above $400 \mathrm{keV}$ between the two systems is evident, despite the better energy resolution of the RSI system. 

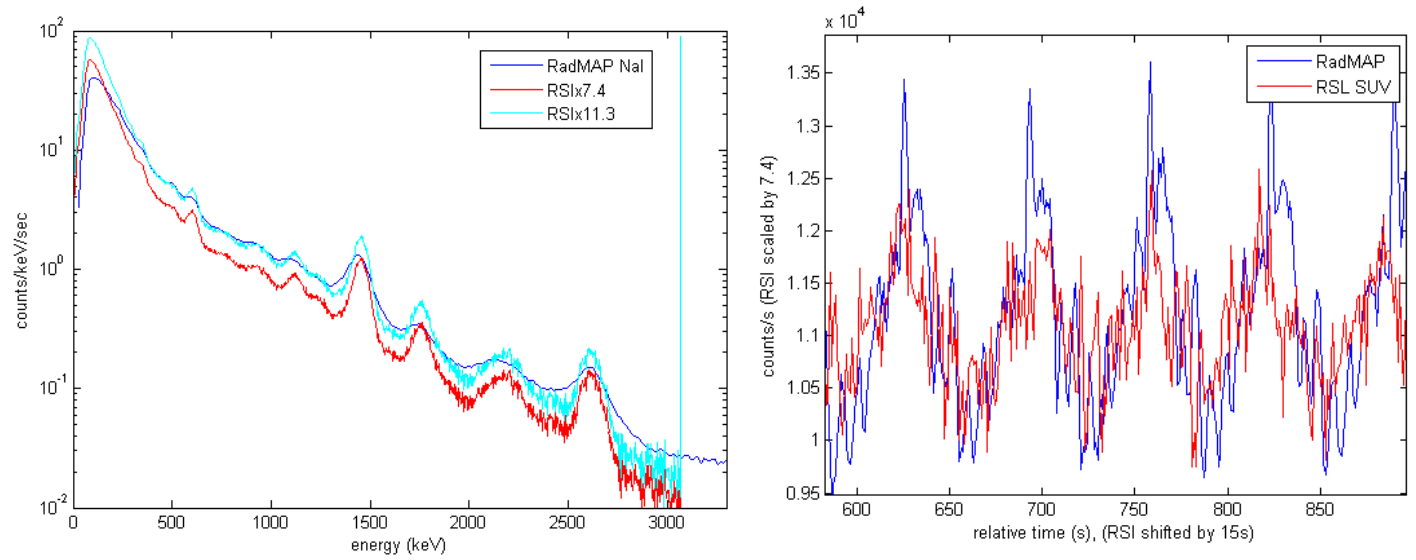

Figure 4.7.30. RadMAP and RSI total gross counts comparisons.
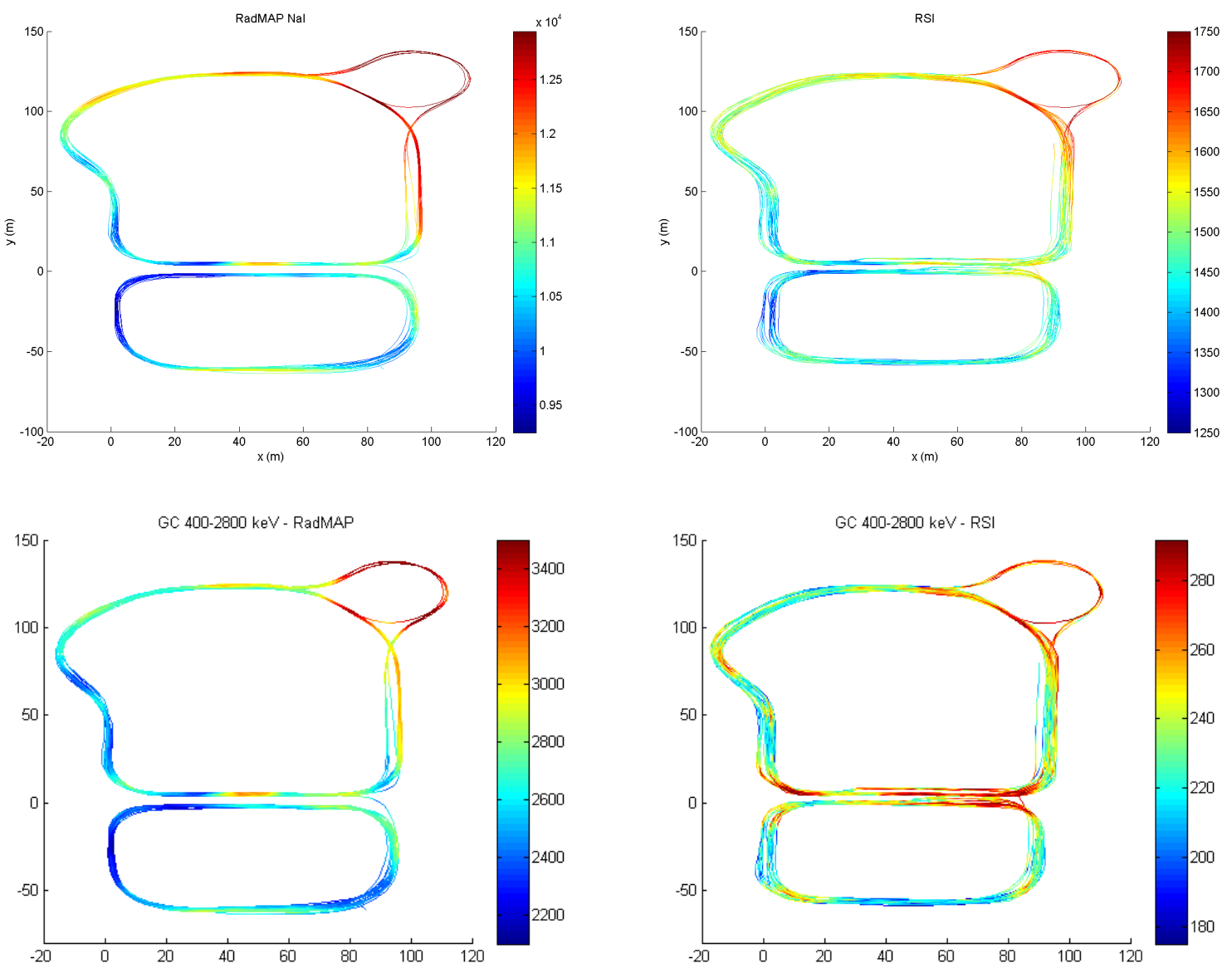

Figure 4.7.31. RadMAP and RSL gross count comparison along the path through FTIG. RadMAP system (left) and the RSL system (right). The top figures are for all energies and the bottom figures are for energies between 400 and $2,800 \mathrm{keV}$. 
The lower plots in Figure 4.7.31 show the positioning of the systems colorized by gross count and colorize rates between 400 and 2,800 keV. Two obvious differences between the RadMAP and the RSL system stand out. The first is that RadMAP rates increase relatively more in the traffic circle than the RSL system, whereas the RSL system appears to produce relatively stronger responses to the hotel during downhill loops and to the church in both directions. The second difference is that the RadMAP system odometry indicates substantially less lateral scatter between laps, particularly along Main St. This is due to the fact that the RadMAP INS positioning results are more accurate and precise than those provided by the GPS fielded on the RSL system. The difference in positioning system performance makes comparisons between the two systems more difficult because the spatial binning (i.e., histogram) method that was used in Section 4.7.2 will produce inaccurate results for the RSL system. However, along Main St., where the measurement activities were focused, dimensionality of the positioning uncertainty by only comparing positions was reduced in the dimension along the roadway, $\mathrm{x}_{\mathrm{M}}$. The RSL $1 \mathrm{~s}$ time bin is then determined, which is the time interval in which the RadMAP system was previously closest to the RSLreported positions. Using those times, the RadMAP list-mode data is binned and compared to the RSI count rates. These comparisons are shown in Figure 4.7.32 for Day 1 laps that the RSL system conducted while trailing RadMAP. The observed rate scaling for the full spectrum $(7.4 \times)$ and for the $400-2,800 \mathrm{keV}$ $(11.3 \times)$ range were applied to the RSI system to plot alongside the RadMAP data. The ordinates are kept equal for the full spectrum plots, and the $400-2,800 \mathrm{keV}$ plots also all have the same range, which is same fractional range as for the full spectrum (i.e., Rate $_{\max }=1.9$ Rate $_{\min }$ ). Throughout, the better statistics generated by RadMAP are evident, but the rate distributions across the figures appear to trend very similarly. However, the RSL system demonstrates an appreciable uptick in count rates in the $\mathrm{x}=0-15 \mathrm{~m}$ range that RadMAP does not. This may be due to the fact that the RSI detectors in the RSL system are both closer to the ground and less shielded from ground emissions than the RadMAP detectors. The 0 $15 \mathrm{~m}$ range corresponds to the observed rate increase by $\mathrm{NaI}(\mathrm{Tl})$ static detectors and shielded HPGe detectors that were described earlier in this report.

Both spectroscopically and with respect to time-dependence, the RadMAP system generates radiological data that are qualitatively very similar to the RSL-fielded capability. However, the RSI detectors have better energy resolution, whereas RadMAP generates appreciably better counting statistics. In some instances radiological changes from the roadway composition will not influence RadMAP as much as the RSL system. 

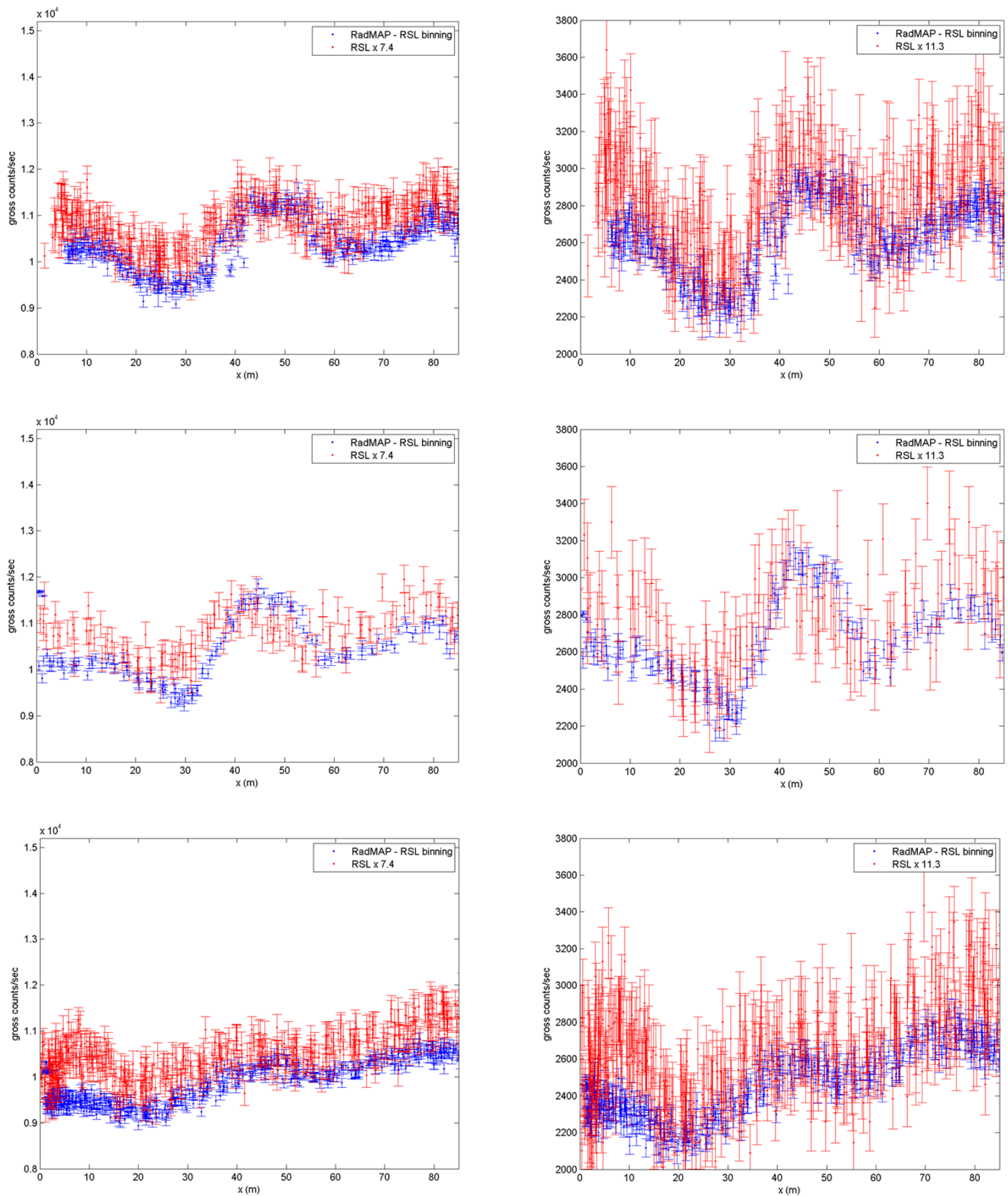

Figure 4.7.32. RadMAP and RSI gross count and algorithm comparison along Main St. Comparisons between (left) gross counts for the RadMAP and RSI systems along Main St. for (top) the loop A uphill laps, (middle) the loop A downhill laps, and (bottom) the loop B downhill laps.(Right) Same data except in the energy range of $400-2,800 \mathrm{keV}$. Note, for full spectrum gross counts, RSI data is scaled by 7.4 , and for the $400-2,800 \mathrm{keV}$ energy range RSI is scaled by 11.3 . 


\subsubsection{RadMAP Radiological Inversion Progress}

A primary goal for fielding RadMAP as part of the MUSE-1 measurement campaign was to collect data in a ground-truth environment such that the Lawrence Berkeley National Laboratory team could establish and test a means of performing a radiological inversion, where each surface that is measured within a scene is attributed an expected emission signature that is derived from a combination of the observed radiological signatures and the known measurement geometry. The intention is to accomplish this spectroscopically, but for an initial example, the method relating to gross counts is summarized below.

First, the observed geometric scene must be digitized and segmented. The LIDAR-based SLAM algorithm described in Section 4.7.5 effectively provides both a digital map and the orientation of the RadMAP truck within that map. Video imagery is used to segment the scenes. Segmentation is important because scenes must be discretized and by using segmentation, the discrete voxels for each large object can be grouped together to improve statistics, thereby making an ill-posed problem more tractable.

Segmentation begins by creating a single image that covers the entirety of the solid angle surrounding RadMAP $(4 \pi)$ out of the separate Ladybug cameras. Each Ladybug system has five cameras that are first stitched to generate $4 \pi$ images; however, because there is not a down-looking camera on the Ladybugs, there is a blind region directly below the cameras. Concurrent panoramic images from each Ladybug system are shown in Figure 4.7.33. The corner of the truck bed is visible in each image, as are roadways and buildings in the CACTF facility.

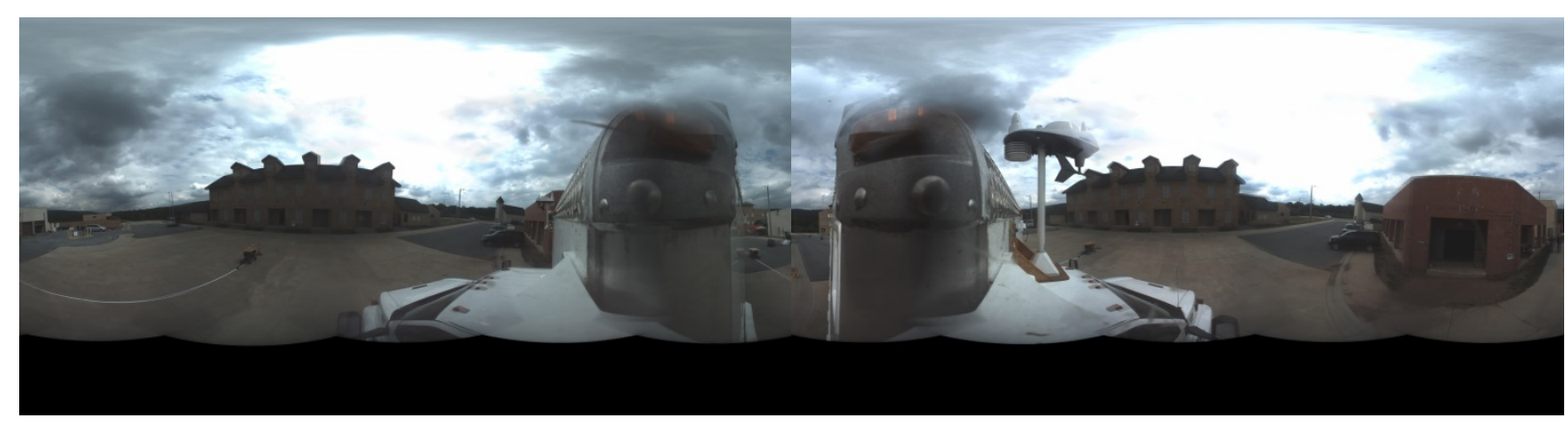

Figure 4.7.33: RadMAP panoramic image from port and starboard Ladybug cameras.

The two panoramic video images are stitched together to make a single image such as the one in the left image of Figure 4.7.34. The images show the forward-facing aspect of the still-frame as the center of the image and the backward-facing portion of the image is on both the left and right sides of the image. Likewise, straight up is the top of the image and straight down is the bottom of the image. Due to the different positions of the two Ladybug systems, there is a certain amount of parallax visible in the center of the image, particularly where the image content is nearest the cameras, which is usually the roadway directly in front of RadMAP. The stitched panoramic images are then automatically segmented using the simple linear iterative clustering (SLIC) algorithm with the goal of facilitating the selection of object boundaries as shown in the right image in Figure 4.7.35. 


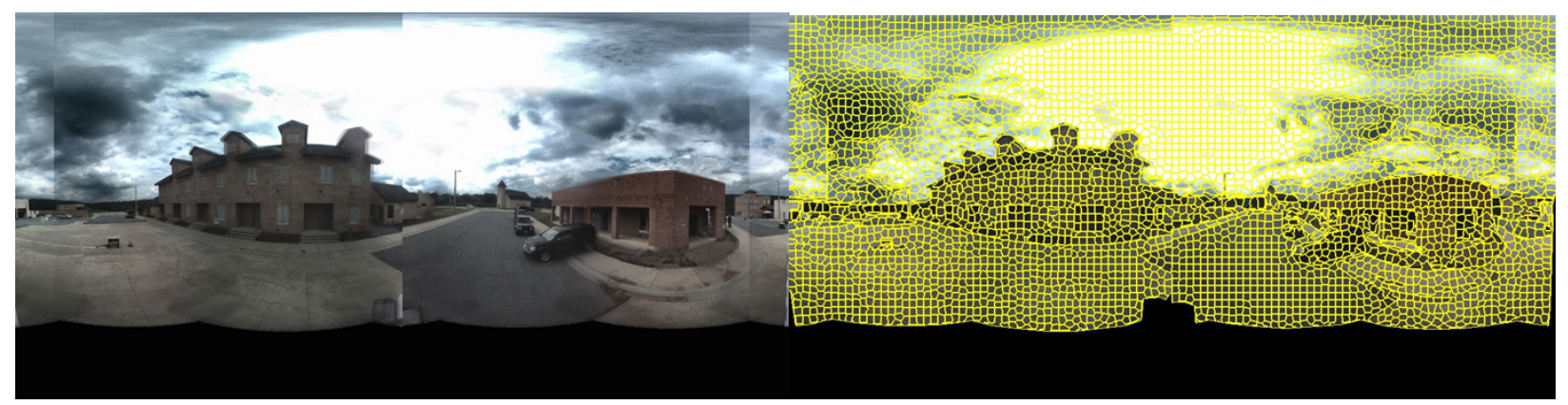

Figure 4.7.34. RadMAP imaging system automatic segmentation methodology. (Left) Stitched panoramic image.(Right) The same image with SLIC automatic segmentation edges overlaid.

For training, a subset of the data is manually labeled. The top-left image of Figure 4.7.35was labelled as indicated in the top-right image. The automatic segmentation was applied to the top-left image and those segments that contained a mixture of classes were omitted from the training set (and are colored black) in the image in the bottom left of the figure. The training dataset is used to train a random forest classifier, which then produced the image shown in the bottom-right of the figure.
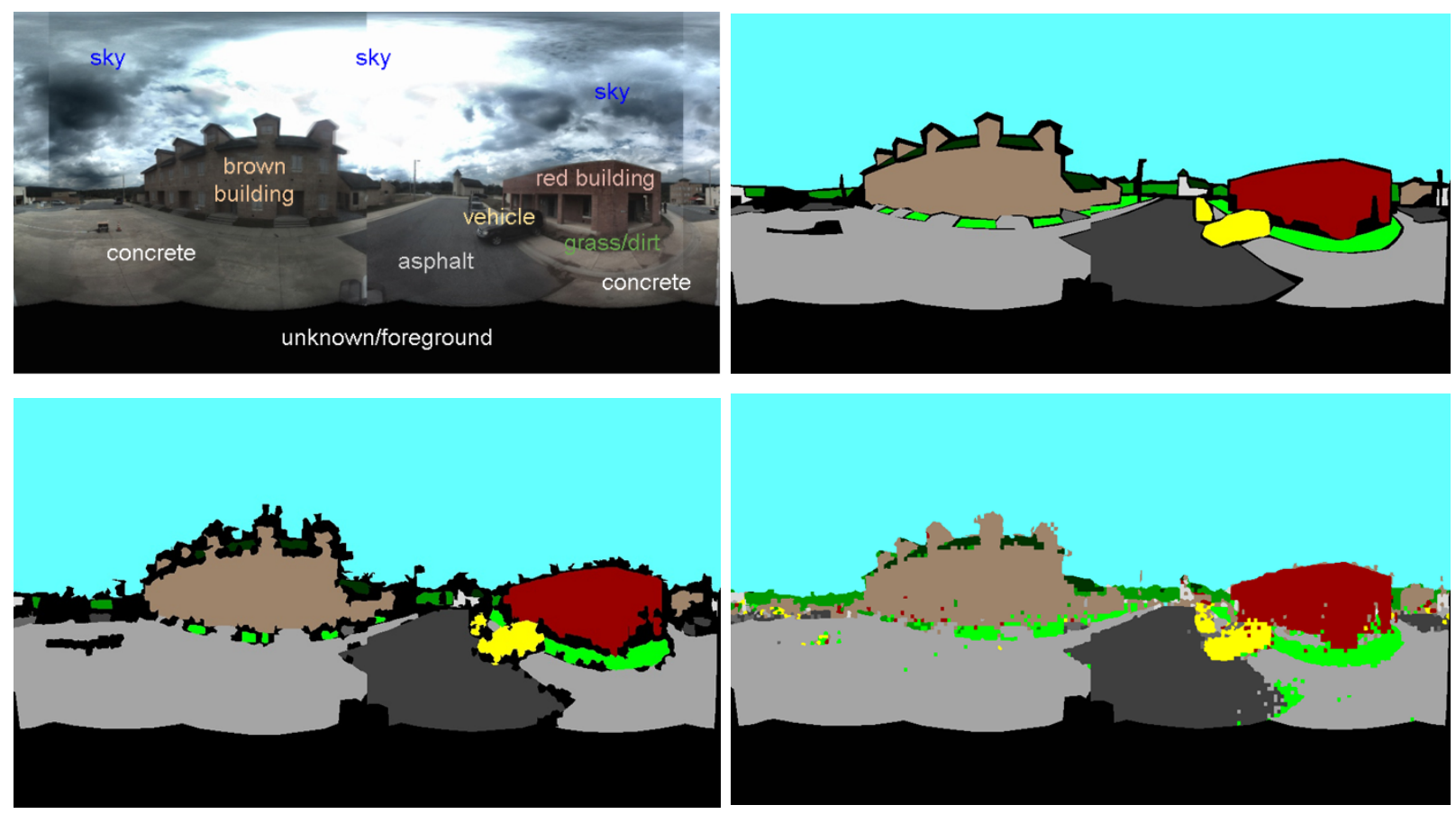

Figure 4.7.35. Illustration of training image system with materials. (Top left) Panoramic image with overlaid manual labeling of objects within image.(Top right) product of manual labeling. (Bottom left) The segmented version of the labelled image, those segments with a mixture of labels are omitted from the training dataset and colored black. (Bottom right) the output of the training algorithm on this same frame.

The random forest classifier was then applied to segments within a 3-Hz sampling of the stitched panoramic still frames, this produces a time-dependent function of the fraction of the solid angle that is subtended by each of the identified classes. The contents of the foreground, or the area beneath the camera system FOV, are also estimated by comparing adjacent still-frames. This is indicated in the top image of Figure 4.7.36. The second image also uses adjacent frames to impose stability on the classification outputs within the FOV. 

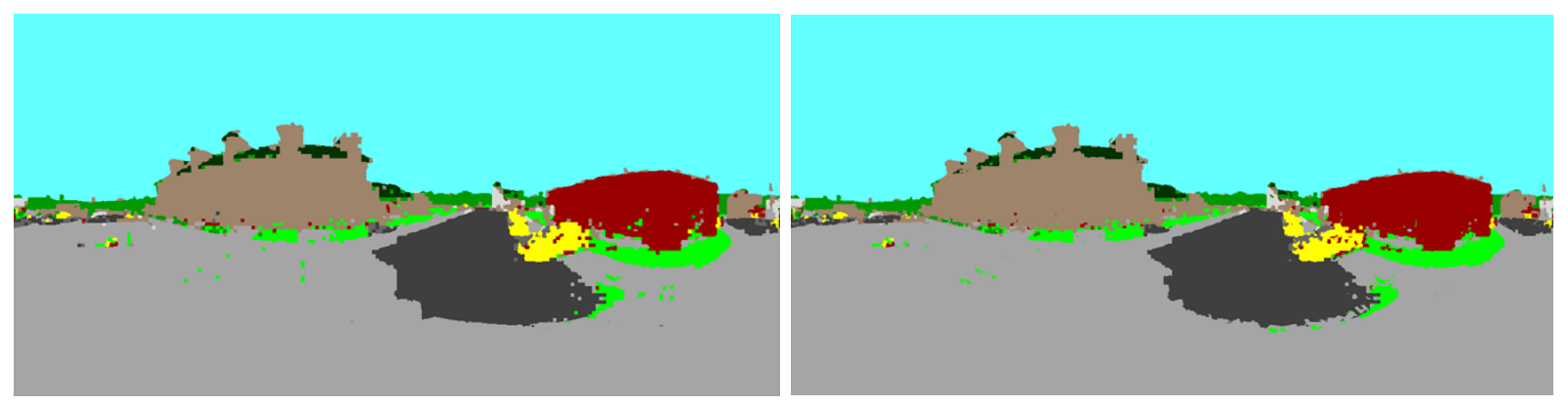

Figure 4.7.36. Illustration of pre- and post-foreground estimation for imaging system. (Left) Same still-frame as in Figure 4.7.35 with estimated foreground content.(Right) Same data with frame-by-frame classification stability algorithm applied.

A single measurement, $y$, can be considered the sum over the response to all image segments, $i$, where the contribution of each segment is the product of the activity per area $\left(a_{i}\right)$, solid angle $\left(\Delta \Omega_{i}\right)$, detector effective area, $A_{i}$, and air attenuation due to a distance $\left(r_{i}\right)$ and an attenuation coefficient, $\mu$. By assuming that each class, $C$, represents the same per-area activity $\left(a_{C}\right)$, the first expression is rewritten below as a sum over segments within each class. Then the "response" due to a single class can be calculated as a time-dependent function, $x_{C}$. For now, air attenuation has been ignored $(\mu=0)$, and a cosine functional form has been assumed for $A$.

$$
\begin{aligned}
y & =\sum_{i} a_{i} \Delta \Omega_{i} A\left(\hat{r_{i}}\right) e^{-\mu r_{i}} \\
& =\sum_{C} a_{C} \sum_{k \in C} \Delta \Omega_{k} A\left(\hat{r_{k}}\right) e^{-\mu r_{k}} \\
& \equiv \sum_{C} a_{C} x_{C}
\end{aligned}
$$




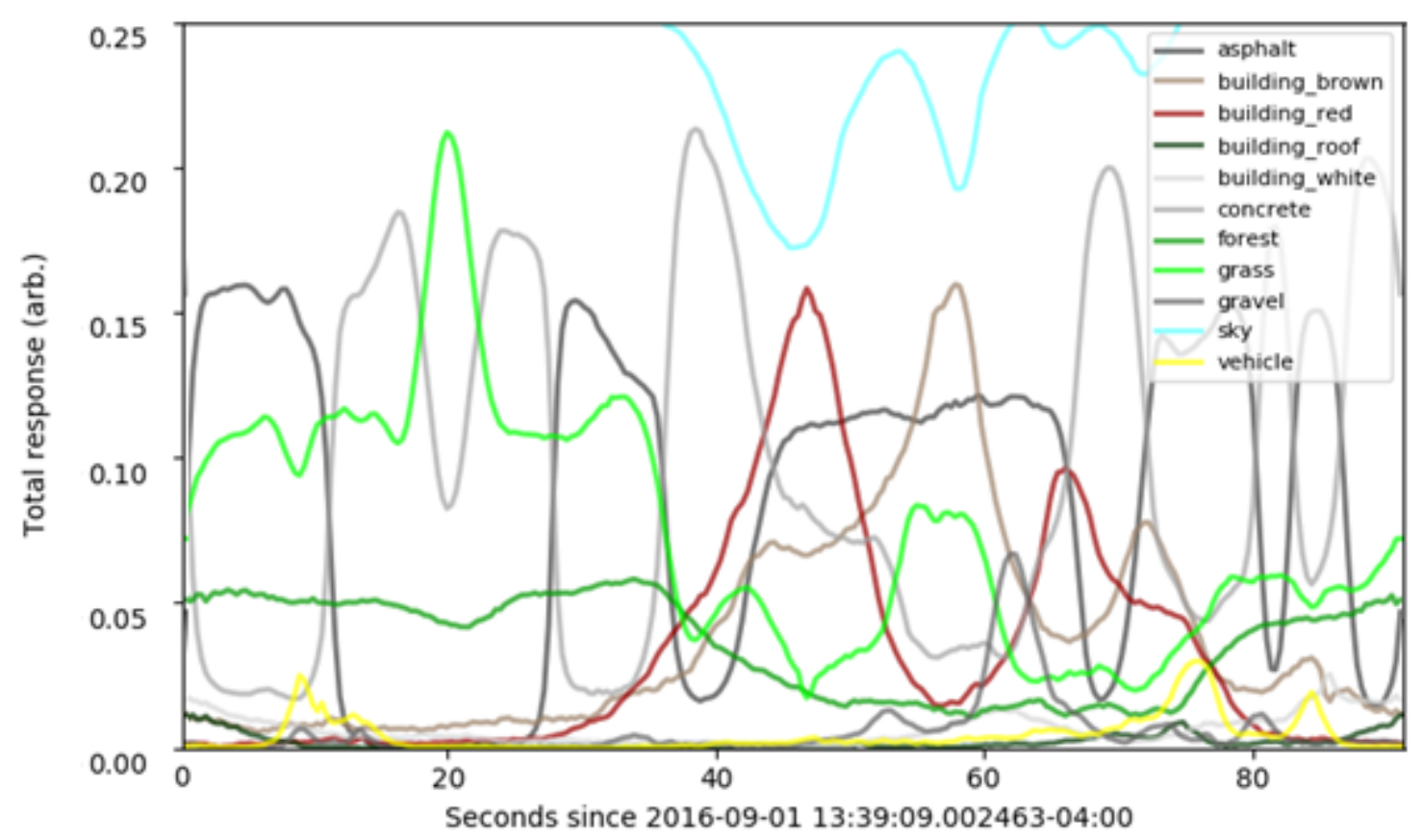

Figure 4.7.37. Response for the indicated classes versus time for a single loop A lap through the FTIG facility.

The best values for $a_{C}$ are then determined through least-square minimization, resulting in the relative activities indicated below. The relative rates scale quite similarly to the HPGe-observed ${ }^{40} \mathrm{~K}$ rates summarized in Section 4.1.

$$
\begin{aligned}
y & =44200 x_{\text {grass }} \\
& +27000 x_{\text {concrete }} \\
& +26000 x_{\text {asphalt }} \\
& +16800 x_{\text {gravel }} \\
& +14700 x_{\text {red }} \\
& +14200 x_{\text {brown }} \\
& +7300 x_{\text {white }}
\end{aligned}
$$




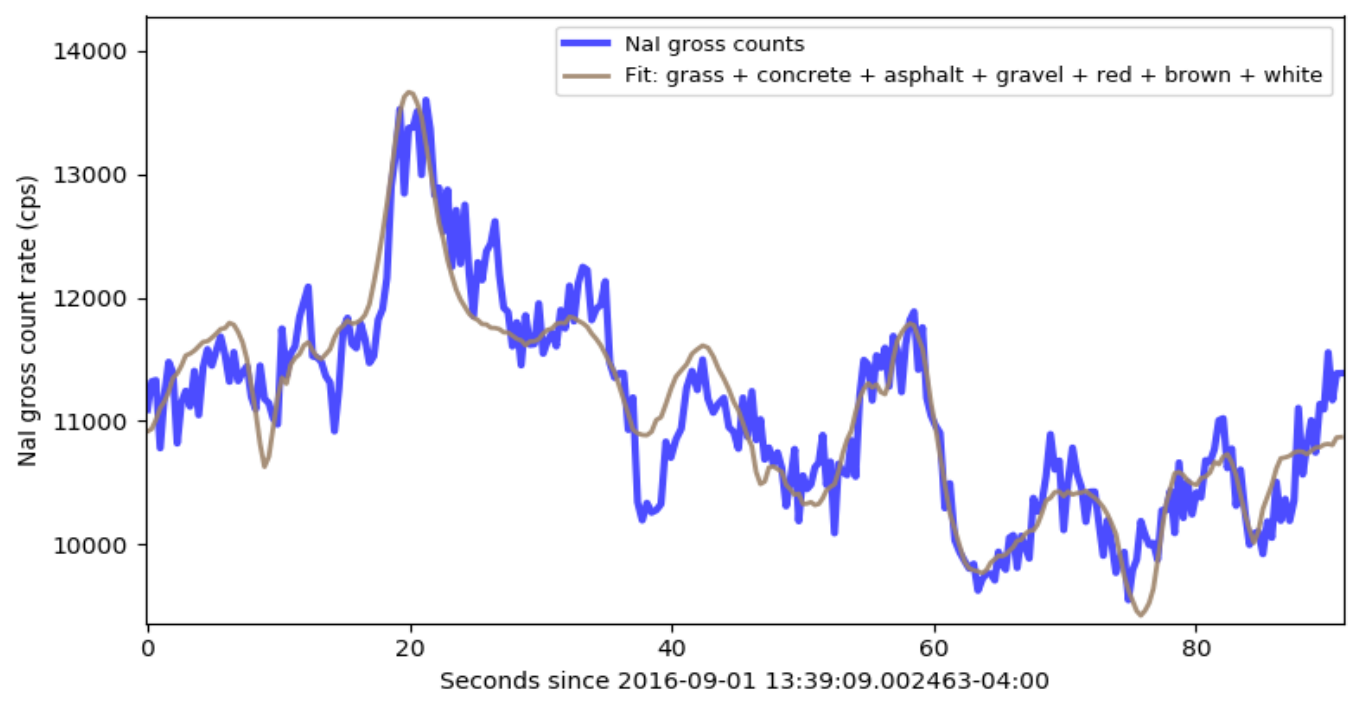

Figure 4.7.38. Best-fit response of measured background and calculated from imaging.

The plan forward is to incorporate measured and simulated detector response function, rather than simple approximations and to use LIDAR-derived data to incorporate true ranges to enable air attenuation calculations. Future efforts will also perform spectroscopic decompositions, wherein each observed gamma-ray energy is probabilistically attributed to natural background components, rather than just gross counts. Using this additional information along with analyzing multiple passes and static measurements, a better understanding of the precision and accuracy of the radiological inversion process can be as a function of various driving conditions.

\subsubsection{RadMAP Hyperspectral Data Collection}

Throughout RadMAP measurements at CACTF, the hyperspectral imagery (HSI) cameras were operational. The cameras are described in some detail in Ref [12], but to summarize, there are two cameras that both record one-dimensional images onto planar CCDs. The second dimension on the CCD records different wavelengths using a prism to diffract the incident light. The cameras are oriented to collect those images along the approximate roll axis of the truck (i.e., vertically), the translation of the truck enables a second dimension to be imaged, although when the truck turns, the suspension system results in small roll changes that distort the raw observed data and are visible in the images shown below. This distortion can be corrected for through integration of the odometry data of the system, but that is not shown here.

One HSI camera, the Resonon Pixa II or 2i, collects light into 240 wavelength channels between 380 and $900 \mathrm{~nm}$ and into 640 spatial bins that span $43.5^{\circ}$. The near infrared (NIR) camera is a Resonon that collects 145 wavelength channels between 900 and $1,700 \mathrm{~nm}$ and into 320 spatial pixels spanning $90^{\circ}$. Both cameras are triggered by hardware at a $45 \mathrm{~Hz}$ rate. Typical mean spectra are shown in Figure 4.7.39. 

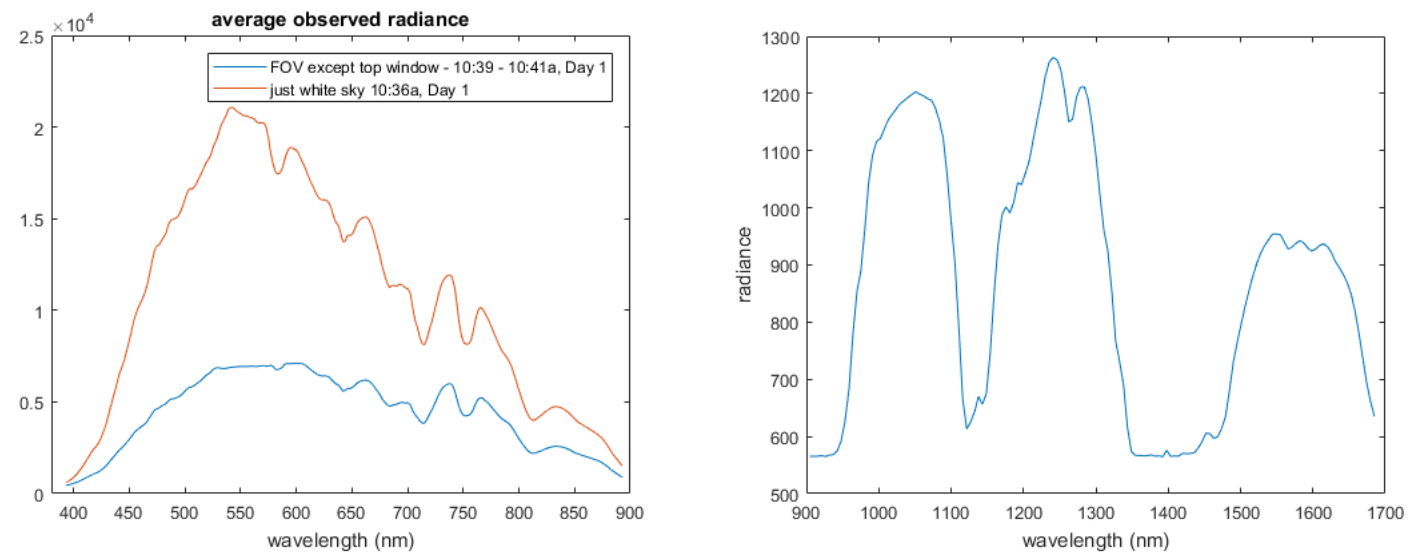

Figure 4.7.39. Example mean spectrum observed in the hyperspectral imaging system. (Left) $2 \mathrm{i}$ camera mean spectrum in the FOV and the mean white-sky spectrum.(Right) the NIR camera mean spectrum in the sky portion of the FOV.

Because the cameras are not uniformly sensitive and the illumination of measured surfaces can vary, typically the observed radiance is converted into reflectance by normalizing each observed spectrum by a nominal white spectrum. The two sky spectra in Figure 4.7.39 were used as the normalizing spectra; however, the NIR camera demonstrated nonuniform sensitivity across the height/roll dimension of the CCD. In attempt to correct for this nonuniformity, the average relative sensitivity of each vertical pixel was estimated and a "mean white image" was generated for the entire CCD. The overall mean NIR image is shown in the left plot of Figure 4.7.40, and the estimated white background image is shown in the right plot.
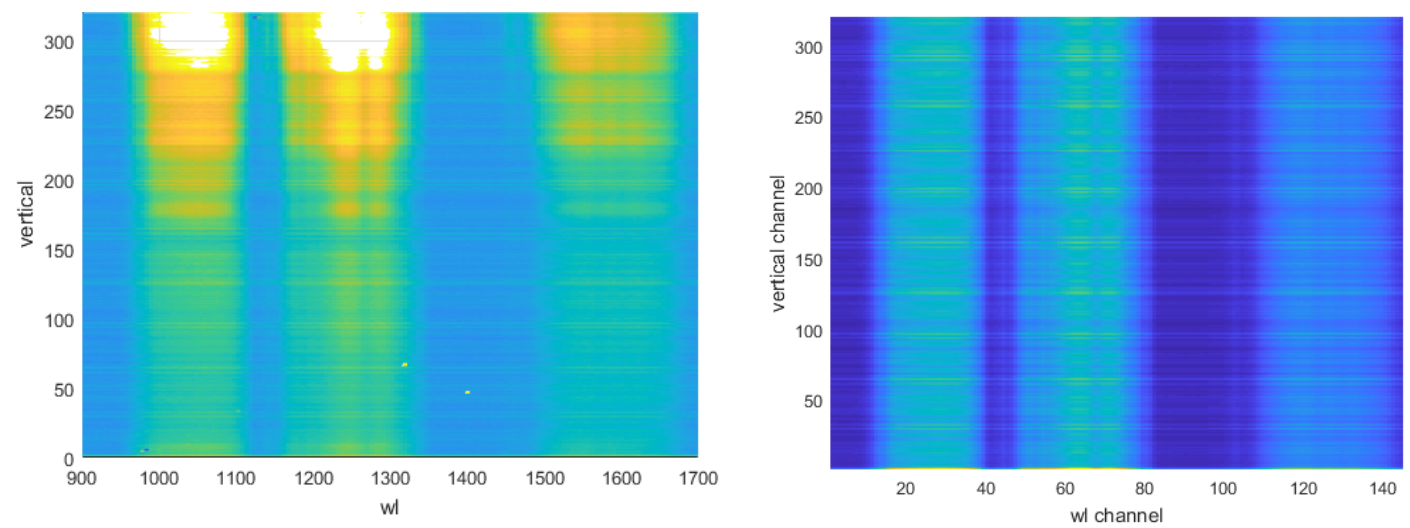

Figure 4.7.40. RadMAP NIR system output image. Mean image from 5,000 frames of NIR data while the system is moving is shown in the left figure. The brightest channels have consistent view of the mostly cloudy

sky. They were selected to create the mean white spectrum shown in the right side of Figure 4.7.39. This function was cross multiplied by the relative responsiveness of the vertical pixels as estimated from the bands of uniform responsiveness in the left figure to generate the right figure.

The average reflectance of the combined average image is shown as the left plot in Figure 4.7.41. The value at a wavelength of $904.8 \mathrm{~nm}$ is highlighted to indicate the smooth transition of the function between the two cameras. 

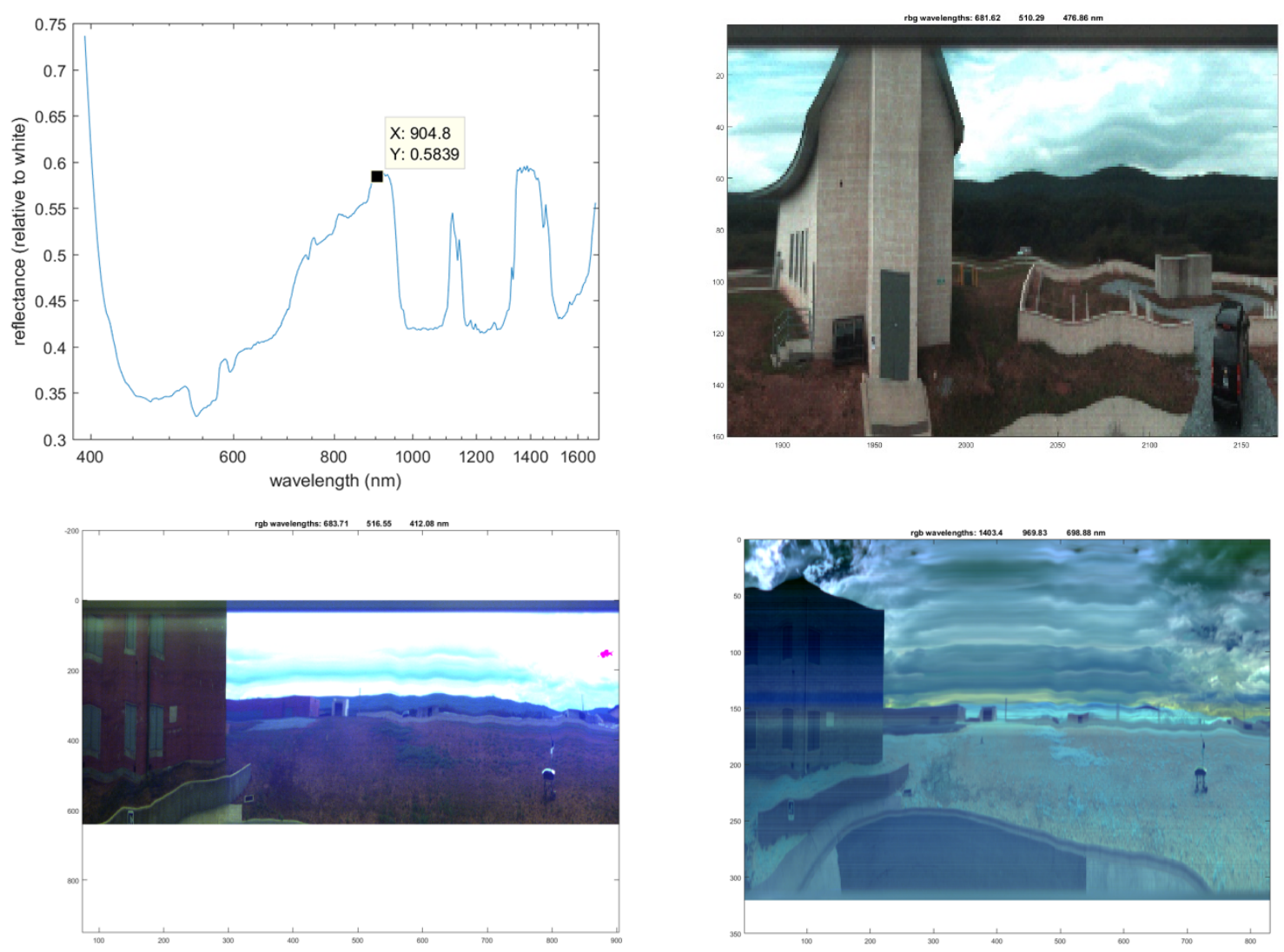

Figure 4.7.41. RadMAP resulting single color image from camera systems. (Top left) Combined average reflectance of the two camera system.(Top right) True-color $2 \mathrm{i}$ image of a portion of a single lap. (Bottom left) Another true-color image, but the axes have been extended beyond the image to represent the same size as the (bottom right) corresponding NIR false colored image.

Given that the $2 \mathrm{i}$ has a reduced FOV, relative to the NIR camera, only the range of spatial pixels in the NIR image that overlap with the $2 \mathrm{i}$ are initially selected and subsequently down-selected the $2 \mathrm{i}$ pixels by 1/4. It is also observed that the two cameras were not aligned in a perfectly coplanar fashion. Instead, the NIR camera was pointed slightly aft of the $2 i$, and this results in the camera viewing different objects at the same time. Specifically, as the distance for an object increases, the NIR camera will see the object later and later. The ideal way to correct for this would be to attribute to each voxelized object within the FOV a $2 \mathrm{i}$ and NIR spectrum separately, and to subsequently combine them spatially, rather than attempting to combine the spectra in time, as has been done here. Despite this, the latter approach works reasonably well. The two cameras appear to share a focal plane that is $10 \mathrm{~m}$ from the system if the NIR is delayed by $90 \mathrm{~ms}$ (or 4 frames). That was done in the following.

Using mean-centered combined reflectance spectra for each pixel at a series of times, singular value decompositions (SVDs) were performed across a few ways of sub-selecting the combined spectra. The SVD is a dimensionality reduction that produces orthonormal spectral eigenfunctions, $U$, that multiply a diagonal matrix of eigenvalues, $S$, and an orthonormal set of eigen-images, $V$ such that the initial set, $M=\mathrm{U} \mathrm{S} \mathrm{V}^{\mathrm{T}}$. The initial set of $M$ contained the entirety of the combined data and indicated that the lowest wavelength portion of the spectrum appeared to provide a major component to the data, which appeared to be mostly noise. This set will not be considered henceforth, and instead a second SVD was run with 
only the spectral components greater than $444 \mathrm{~nm}$ included in $M$ (cutting off the 25 shortest-wavelength spectral bins), and another SVD wherein also the upward facing spatial channels (e.g., above $3.6^{\circ}$ above the horizontal roll axis) were removed from $M$ were conducted. The magnitudes of the top $20 \mathrm{SVD}$ eigenvalues are shown in the left plot in Figure 4.7.42, though the eigenvalues for the set that include the sky channels are divided by two. This plot indicates that the components have similar relative strength, although there appears to be appreciable drop-off in the eigenvalue at the eighth component for the ground-facing set, whereas the eigenvalues of the sky-containing set continue to weaken in strength for several more eigenfunctions. The right plot in Figure 4.7.42 shows the top eight eigenspectra for the two sets. The large response near $900 \mathrm{~nm}$ for component eight in the ground-facing spectra is further indication that an appreciable aspect of this component is noise. Similarly, the amount of oscillation between 500 and $700 \mathrm{~nm}$ for several of the sky-facing set's components likely indicates the difficulty in comprising all the spectral variability into orthogonal components.
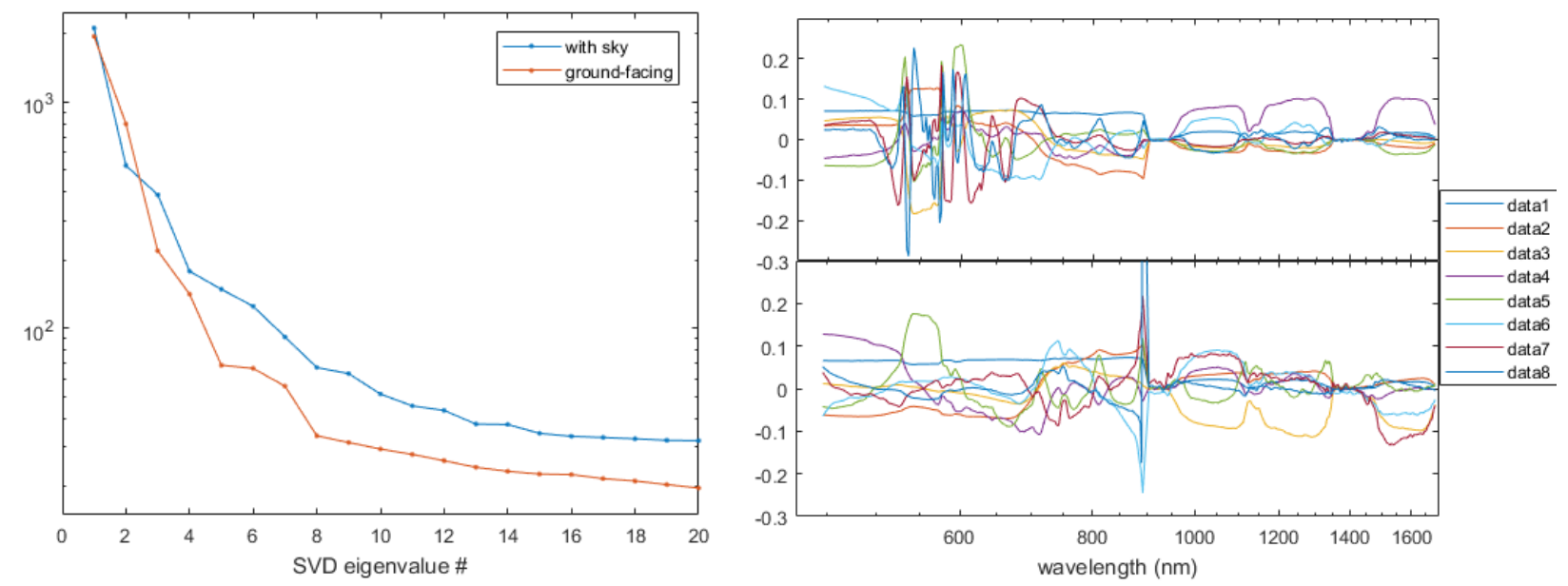

Figure 4.7.42. Single value decompositions of measured spectrum with resulting wavelength. (Left) Eigenvalues for the first 20 principal components for the two SVDs.(Right) The top eight spectral eigenvectors from the SVDs. The top plot is for the SVD with the sky included, whereas the bottom is comprised predominantly of terrestrial-reflected light.

The eigen-images for the first 12 principal components for the with-Sky SVD are shown as Figure 4.7.43, and the first nine eigen-images for the ground-facing SVD are shown as Figure 4.7.44. For both images, the lower-order (high number) components begin to show appreciable noise. In the sky-facing set, the noise appears as horizontal streaks, which is apparently residual from the NIR camera that was supposed to be removed as described above. Conversely, the ground-facing SVD set indicates substantial white noise (e.g. pixel-by-pixel noise) which is anticipated because the 8th and 9th components appear to sit very near the noise floor in the eigenvalue spectrum. 

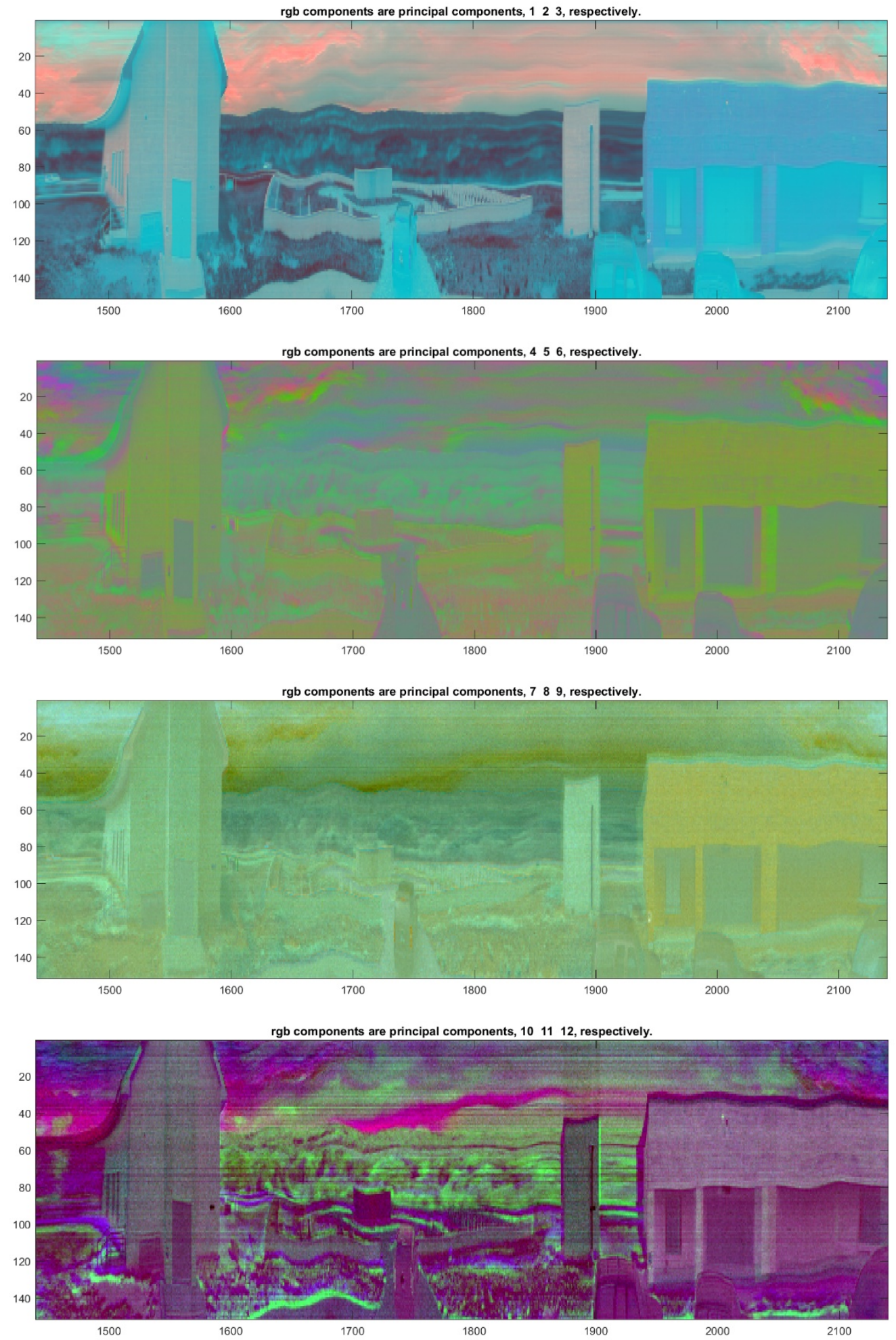

Figure 4.7.43. False-color images for the eigen-images of the first twelve principal components of sky-facing SVD. The R,G,B values are the indicated principal components for each image. 

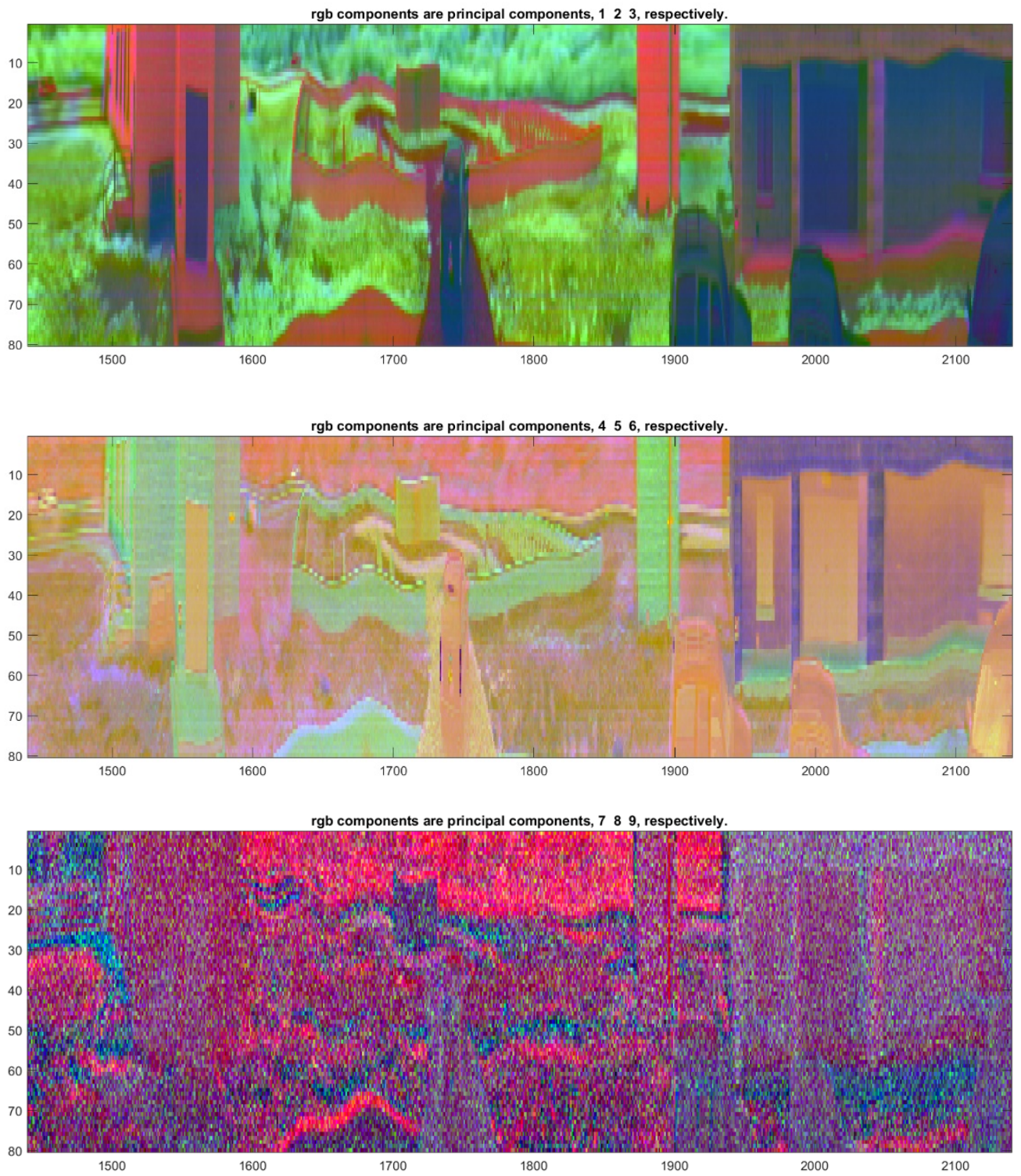

Figure 4.7.44. False-color images for the eigen-images of the first nine principal components for the groundfacing SVD. The R,G,B values are the indicated principal components for each image.

In principle, a dimensionality reduced set of HSI image components could be used to classify various objects within a collected image. Given the relative homogeneity of the construction materials that are visible at the FTIG facility, the primary resulting classifications from HSI imagery of said materials is primarily given by the lighting and visible coloring of the materials, which although not particularly interesting, would be useful to test and benchmark a more robust classification method based on the use of reconstructed 3-D models and knowledge of the illumination conditions This more robust classification could be used to improve the video-based segmentation that was described in Section 4.7.7.

\subsubsection{RadMAP Measurements Summary}

The RadMAP system was successfully fielded at the FTIG CACTF site, operating three types of ionizing radiation detector systems, real-time alarming algorithms, and a suite of contextual sensors. Sections 4.7.1, 4.7.2, and 4.7.4 directly demonstrate the system's synchronization, and the entirety of Section 4.7 
summarized and demonstrated various analyses that can be brought to bear on the RadMAP data to bring better understanding to both the environment that was being measured and how it has impacted the observed radiological signatures. Comparisons between RadMAP NaI(Tl) signatures and those obtained by a fielded capability demonstrate qualitative similarities, though RadMAP obtains significantly better counting statistics. Although the RadMAP NaI(Tl) array has worse energy resolution than the RSI system, the additional HPGe detectors enable spectral ambiguities to be resolved so that the energy resolution is unproblematic.

It remains to be seen how folding both this contextual information and better spectroscopic decomposition algorithms into the radiological inversion analyses will impact the ability to determine surface emission rates for various background components. However, the initial findings summarized in Section 4.7.8 qualitatively demonstrate that RadMAP is capable of providing material activities with a fidelity that could be useful in some applications.

\subsection{WIND | EMAPS | AIPT MEASUREMENTS}

Three different backpack systems were used during MUSE 1 to measure background radiation. ORNL evaluated the DNDO Wearable Intelligent Nuclear Detection (WIND) government reference system, Johns Hopkins Applied Physics Laboratory (APL) used the EMAPS backpack, and NA84 PackEye backpack. The goal was to evaluate the backpacks with radiation sources, but because of logistic issues, measurements were performed with background only. As a result of the lack of data from the other systems, the ORNL WIND system shown in Figure 4.8.1 is the focus of this analysis.
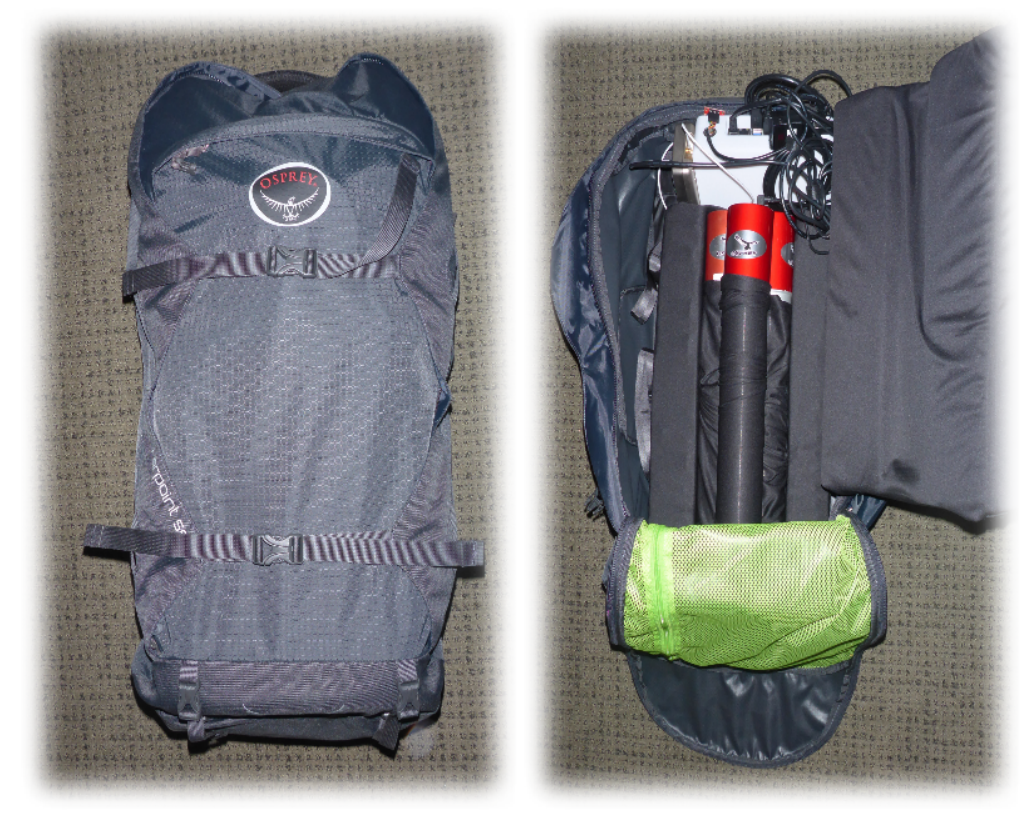

Figure 4.8.1. DNDO WIND backpack radiation detection system prototype.

The backpack was carried for four passes at nominal walking speed, and the paths taken for these passes are shown in Figure 4.8.2 with the resulting count rates along this path shown in Figure 4.8.3. This data was shared with participants of the DNDO WIND project for background analysis in algorithm development for this detector configuration. 


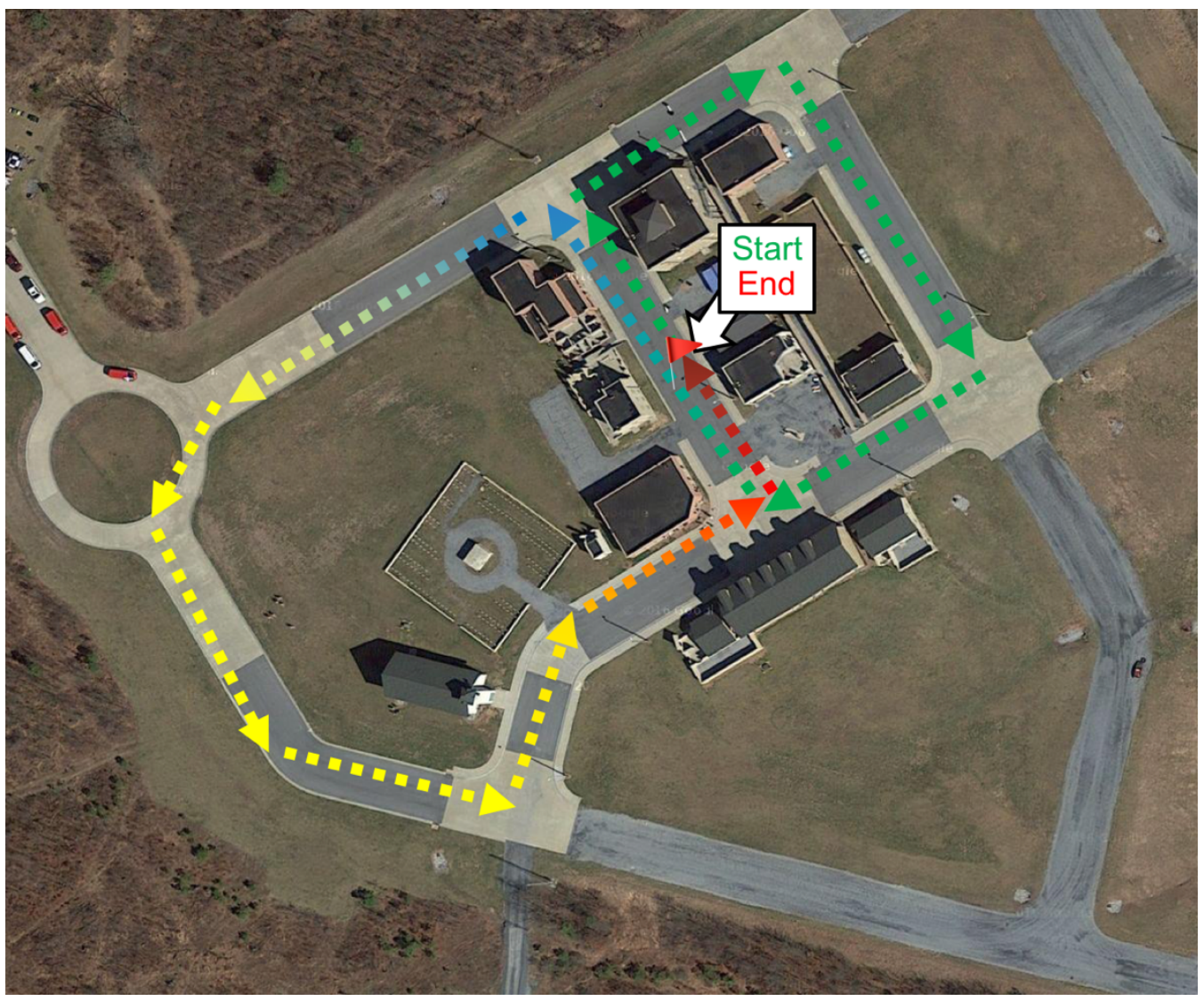

Figure 4.8.2. Measurement path for WIND data collections. 


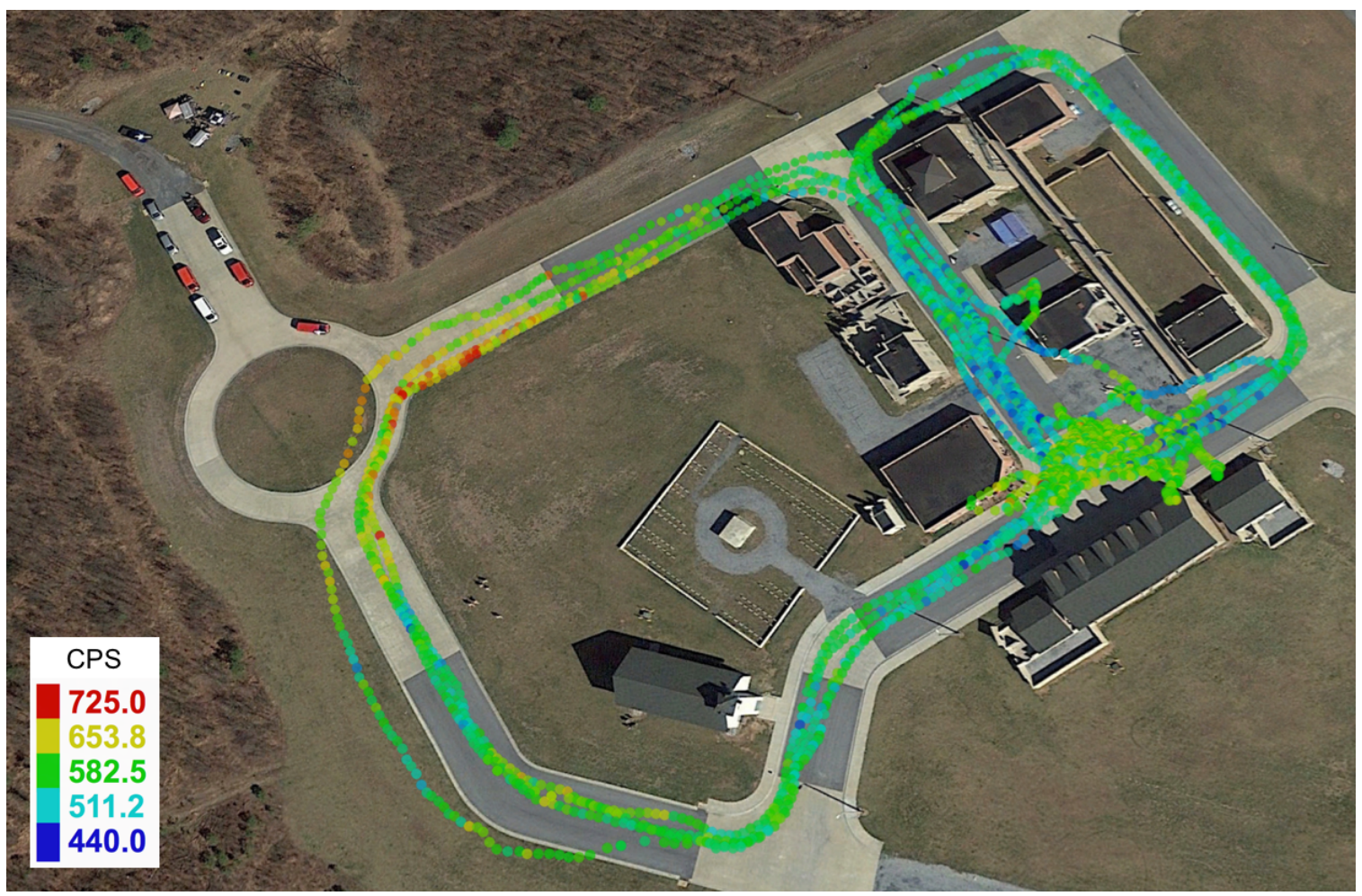

Figure 4.8.3. Count rates for measurements taken with the WIND backpack. 


\section{REFERENCES}

[1] A. Iyengar, M. Beach, R. J. Newby, L. Fabris, L. H. Heilbronn, J. P. Hayward, "Systematic Measurement of Fast Neutron Background Fluctuations in an Urban Area Using a Mobile Detection System," Nuclear Instruments and Methods in Physics Research Section A: Accelerators, Spectrometers, Detectors and Associated Equipment, Volume 773, February 11, 2015, 27-32.

[2] SCALE: A Comprehensive Modeling and Simulation Suite for Nuclear Safety Analysis and Design, ORNL/TM-2005/39, Version 6.1 (2011). Available from Radiation Safety Information Computational Center at Oak Ridge National Laboratory as CCC-785.

[3] D. E. Peplow, "Monte Carlo Shielding Analysis Capabilities with MAVRIC," Nuclear Technology 174(2), 289-313 (2011).

[4] R. J. McConn, Jr., C. J. Gesh, R. T. Pagh, R. A. Rucker, and R. G. Williams III, “Compendium of Material Composition Data for Radiation Transport Modeling," PNNL-15870, Rev. 1, Pacific Northwest National Laboratory, Richland, WA, March 2011.

[5] M. W. Swinney, D. E. Peplow, A. D. Nicholson, and B. W. Patton, "NORM Concentration Determination in Common Construction Materials in an Urban Environment," Transactions of the American Nuclear Society 114, 635-638 (2016).

[6] W. A. Wieselquist, "The SCALE 6.2 ORIGEN API For High Performance Depletion," ANS MC2015- Joint International Conference on Mathematics and Computation (M\&C), Supercomputing in Nuclear Applications (SNA) and the Monte Carlo (MC) Method, Nashville, TN, April 19-23, 2015 .

[7] T. Goorley, M. James, T. Booth, F. Brown, J. Bull, L. J. Cox, J. Durkee, J. Elson, M. Fensin, R. A. Forster, J. Hendricks, H. G. Hughes, R. Johns, B. Kiedrowski, R. Martz, S. Mashnik, G. McKinney, D. Pelowitz, R. Prael, J. Sweezy, L. Waters, T. Wilcox, and T. Zukaitis, "Initial MCNP6 Release Overview, "Nuclear Technology 180, 298-315 (2012).

[8] D. J. Mitchell, L. T. Harding, G. G. Thoreson, and S. M. Horne, "GADRAS Detector Response Function," SAND2014-1946, Sandia National Laboratories, Albuquerque, NM, November 2014.

[9] M. W. Swinney, D. E. Peplow, and A. D. Nicholson, "Characterization of NORM in an Urban Environment using HPGe Measurements and MCNP6 Simulations," Transactions of the American Nuclear Society 116, 917-920 (2017).

[10] S. W. Mosher, S. R. Johnson, A. M. Bevill, A. M. Ibrahim, C. R. Daily, T. M. Evans, J. C. Wagner, J. O. Johnson and R. E. Grove, "ADVANTG-An Automated Variance Reduction Parameter Generator," ORNL/TM-2013/416 Rev. 1, Oak Ridge National Laboratory, Oak Ridge, TN, August 2015. Available from Radiation Safety Information Computational Center as C00831.

[11] M. S. Bandstra, T. J. Aucott, E. Brubaker, D. H. Chivers, R. J. Cooper, J. C. Curtis, J. R. Davis, T. H. Joshi, J. Kua, R. Meyer, V. Negut, M. Quinlan, B. J. Quiter, S. Srinivasan, A. Zakhor, R. Zhang, and K. Vetter, "RadMAP: The Radiological Multi-sensor Analysis Platform" Nuclear Instruments and Methods in Physics Research A 840 (2016) 59-68.

[12] T. H. Joshi, R. J. Cooper, J. Curtis, M. Bandstra, B. R. Cosofret, K. Shokhirev, and D. Konno, “A Comparison of the Detection Sensitivity of the Poisson Clutter Split and Region of Interest Algorithms on the RadMAP Mobile System." IEEE Transactions on Nuclear Science 63.2 (2016): $1218-1226$. 
